NISTIR 6333

\title{
A Generalized Pressure Drop Correlation for Evaporation and Condensation of Alternative Refrigerants in Smooth and Micro-fin Tubes
}

Jun-Young Choi

Mark A. Kedzierski

Piotr A. Domanski

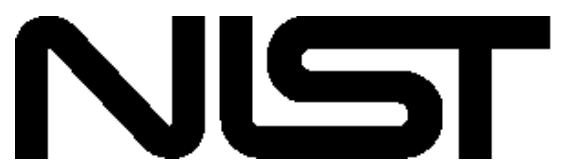

United States Department of Commerce Technology Administration

National Institute of Standards and Technology

Building and Fire Research Laboratory

Gaithersburg, MD 20899 
NISTIR 6333

\section{A Generalized Pressure Drop Correlation for Evaporation and Condensation of Alternative Refrigerants in Smooth and Micro-fin Tubes}

Jun-Young Choi

Mark A. Kedzierski

Piotr A. Domanski

October 1999

Building and Fire Research Laboratory

National Institute of Standards and Technology

Gaithersburg, MD 20899

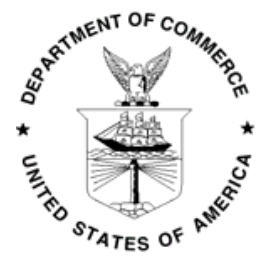

U.S. Department of Commerce

William M. Daley, Secretary

Technology Administration

Gary R. Bachula, Acting Under Secretary for Technology

National Institute of Standards and Technology

Raymond G. Kammer, Director 


\title{
A Generalized Pressure Drop Correlation for Evaporation and Condensation of Alternative Refrigerants in Smooth and Micro-fin Tubes
}

\author{
Choi, J. Y., Kedzierski, M. A., and Domanski, P. A.
}

\begin{abstract}
This paper presents a pressure drop correlation for evaporation and condensation in smooth and micro-fin tubes. The correlation was developed from a database consisting of the following pure and mixed refrigerants: R125, R134a, R32, R410A (R32/R125 50/50 \% mass), R22, R407C (R32/R125/R134a, 23/25/52 \% mass) and R32/R134a (25/75 \% mass). The new correlation was obtained by replacing the friction factor and the tube-diameter in the Bo Pierre correlation with a friction factor derived from pressure drop data for a micro-fin tube and the hydraulic diameter, respectively. The new correlation predicted the measured micro-fin data with an average residual of $10.8 \%$, and it also predicted the pressure drop in smooth tube with an average residual of 15.0 $\%$. In addition, the correlation was used to predict ${ }^{1}$ pressure drop data for refrigerant/lubricant mixtures by using a viscosity-mixing rule. As a result, the new correlation predicted the measured evaporation and condensation pressure drop data for mixtures of various lubricants with R12, R22, and R134a with an average residual of $19.0 \%$.
\end{abstract}

Keyword: alternative refrigerants, correlation, friction factor, heat transfer, hydraulic diameter, micro-fin tube, pressure drop, smooth tube, two-phase flow

\footnotetext{
${ }^{1}$ Certain trade names and company products are mentioned in the text or identified in an illustration in order to adequately specify the experimental procedure and equipment used. In no case does such an identification imply recommendation or endorsement by the National Institute of Standards and Technology, nor does it imply that the products are necessarily the best available for the purpose.
} 


\section{Table of Contents}

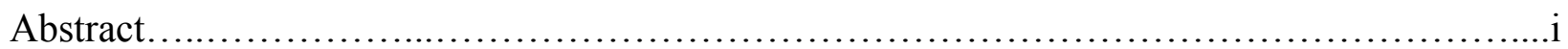

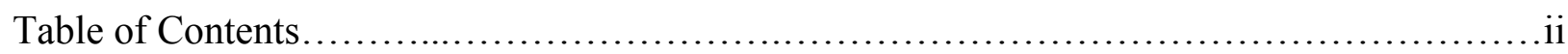

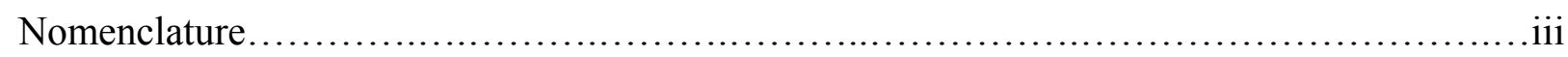

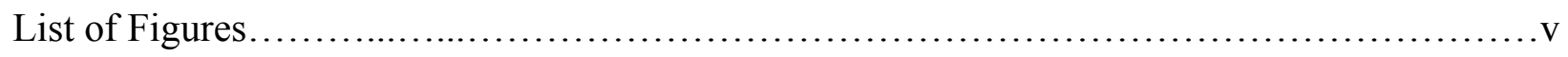

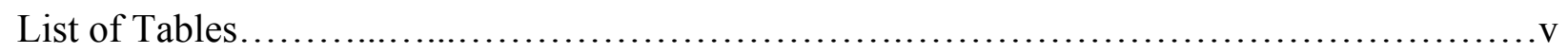

Introduction. .....................................................................

Experiments...................................................................

Refrigerant Property Evaluation..................................................

Comparison of Data to Existing Correlations........................................... 7

Homogenous Flow Models.....................................................8

Separated Flow Models......................................................... 12

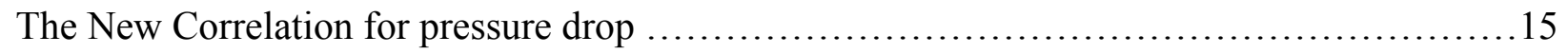

Pure Refrigerants......................................................... 15

Refrigerant/Lubricant Mixtures..............................................20

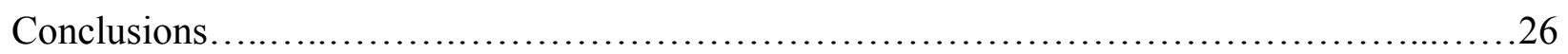

Acknowledgments...........................................................27

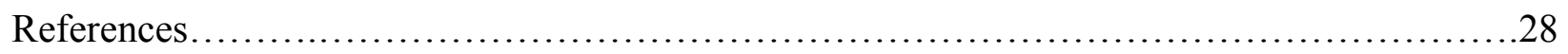

Appendix A...................................................................... 31

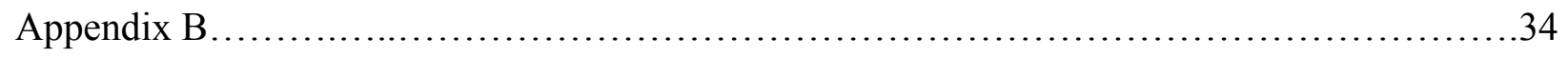

Appendix C.................................................................. 41

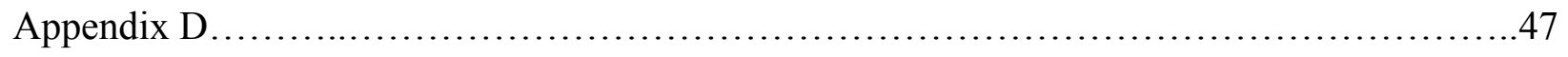

Appendix E................................................................... 50 


\section{NOMENCLATURE}

\section{English Symbols}

$A$ area $\left(\mathrm{mm}^{2}\right)$ or force due to acceleration $(\mathrm{N})$

$A_{c}$ cross sectional flow area $\left(\mathrm{mm}^{2}\right)$

$A_{w}$ cross sectional tube wall area per fin $\left(\mathrm{mm}^{2}\right)$

$C$ constant

$D$ diameter (mm)

$D_{e} \quad$ equivalent diameter of micro-fin tube (mm)

$D_{h}$ hydraulic diameter of micro-fin tube (mm)

$D_{o} \quad$ outside diameter $(\mathrm{mm})$

$D_{r} \quad$ maximum inside diameter of micro-fin tube $(\mathrm{mm})$

$e \quad$ fin height (mm)

$F \quad$ force due to pressure (N)

$f$ friction factor

$G$ mass flux $\left(\mathrm{kg} / \mathrm{m}^{2} \mathrm{~s}\right)$

$g$ acceleration due to gravity $\left(\mathrm{m} / \mathrm{s}^{2}\right)$

$h$ enthalpy $(\mathrm{kJ} / \mathrm{kg})$

$j \quad$ volumetric flux $(\mathrm{m} / \mathrm{s})$

$L$ tube length (m)

$m$ mass flow rate $(\mathrm{m} / \mathrm{s})$

$N$ number of micro fins

$n \quad$ exponent in Chishom two-phase frictional multiplier

$P$ pressure $(\mathrm{Pa})$

$\Delta P$ pressure drop $(\mathrm{Pa})$

$p \quad$ fin pitch : (mm)

$Q \quad$ volumetric flow rate $\left(\mathrm{m}^{3} / \mathrm{s}\right)$

$r \quad$ radius $(\mathrm{mm})$

$S_{p} \quad$ perimeter of one fin and channel taken perpendicular to the axis of the fin (mm)

$T$ temperature

$t_{w} \quad$ tube wall thickness at root of fin $(\mathrm{mm})$

$U, u x$-component velocity $(\mathrm{m} / \mathrm{s})$

$V, v \quad y$-component velocity $(\mathrm{m} / \mathrm{s})$

$V_{f s} \quad$ volume of free space per unit length

$W$ molecular mass

$w$ mass fraction

$x$ mass vapor quality

$z \quad$ length component

\section{Greek symbols}

$\alpha \quad$ fin angle (degree), or void fraction

$\beta \quad$ helix angle (degree)

$\phi \quad$ two-phase frictional multiplier

$\lambda$ constant

$\mu \quad$ viscosity $(\mathrm{N} / \mathrm{ms})$ 
$v \quad$ specific volume $\left(\mathrm{m}^{3} / \mathrm{kg}\right)$

$\pi \quad 3.141592$

$\rho$ density $\left(\mathrm{kg} / \mathrm{m}^{3}\right)$

$\tau$ wall shear stress $\left(\mathrm{N} / \mathrm{m}^{2}\right)$

$\omega$ mass fraction

$\xi \quad$ Yokozeki's factor

$\Psi$ mole fraction

$\underline{\text { Subscripts }}$

B Blausius

BP Bo Pierre

C Collier and Thome

c critical

$\mathrm{f}$ friction or liquid

fo total flow assumed liquid

fg between liquid and vapor

$\mathrm{g}$ vapor

h hydraulic

I component

in inlet

lo liquid phase only flowing

$\mathrm{m}$ mixture

o lubricant

out outlet

$r$ reduced

ref refrigerant

to two-phase in Jung-Radermacher correlation

tot total

TP, tp two-phase

$\mathrm{w}$ wetted

Nondimensional numbers

$\mathrm{K}_{f}=\frac{\Delta x h_{f g}}{L} ;$ two-phase number

$\operatorname{Re}=\frac{G D}{\mu} ; \quad$ Reynolds number

$X_{t t}=\left(\frac{1-x}{x}\right)^{0.9}\left(\frac{\mu_{g}}{\mu_{f}}\right)^{0.1}\left(\frac{v_{f}}{v_{g}}\right)^{0.5}$; Lockhart-Martinelli parameter 


\section{FIGURES}

Figure 1. Schematic diagram of experimental apparatus.

Figure 2. Cross section of test section.

Figure 3. Detail schematic of test section.

Figure 4. Detail cross section of micro-fin tube.

Figure 5. Prediction of NIST pressure drop measurements with Pierre (1964) correlation.

Figure 6. Predictions of NIST pressure drop measurements with homogenous model as given in Collier and Thome (1994).

Figure 7. Prediction of NIST pressure drop measurements with the separated flow model (Eq. 7)

Figure 8. Predictions of NIST pressure drop measurements with Jung-Radermacher (1989) correlation.

Figure 9. Predictions of NIST pressure drop measurements with NIST correlation.

Figure 10. Predictions of Eckels et al. (1993) pressure drop measurements with NIST correlation.

Figure 11. Predictions of Pate et al. (1991) pressure drop measurements with NIST correlation.

Figure 12. Lubricant viscosity as a function of mole fraction.

Figure 13. Reynolds Number for selected refrigerant/lubricant mixtures versus mole fraction.

Figure 14. Predictions of Pate et al. (1991) condensation refrigerant/lubricant pressure drop measurements with NIST correlation.

Figure 15. Predictions of Pate et al. (1991) evaporation refrigerant/lubricant pressure drop measurements with NIST correlation.

\section{LIST OF TABLES}

Table 1. Geometric parameters of micro-fin tube.

Table 2. Summary of test conditions.

Table 3. Geometric tube parameters of Pate (1991) and Eckels (1991 and 1993).

Table 4. Summary of test conditions for Pate (1991) and Eckels (1991 and 1993).

Table 5. Refrigerant/Lubricant mixture tests by Pate et al. (1991). 


\section{INTRODUCTION}

For approximately 50 years, automobile and residential air-conditioners have used smooth tubes and refrigerants R12, and R22 with a small fraction of lubricant in the refrigerant. Consequently, the smooth tube pressure drop has been studied extensively for R12 and R22. Today, due in part to government efficiency requirements and in part to competition, approximately $50 \%$ of new evaporators and condensers in residential air-conditioning equipment employ enhanced micro-fin tubes rather than smooth tubes. Further changes in the air-conditioning industry have been imposed by the ozone crisis, which has led to the introduction of alternative refrigerants to replace R12 and R22. For these reasons, a correlation to predict the pressure drop for alternative refrigerants and refrigerant/lubricant mixtures in smooth and micro-fin tubes is required to design modern heat exchangers.

In the ten years since the identification of the ozone crisis, few publications on pressure drop are available for micro-fin tubes with alternative refrigerants. Most of the available pressure drop data for alternative refrigerants is for R134a. For example, Koops (1992) provides experimental data for the R134a condensation pressure drop in a micro-fin tube. His data showed that the pressure drop for R134a was greater than that for R12. Eckels and Pate (1991) and Fukushima and Kudou (1990) found that R134a/lubricant mixtures exhibited a greater evaporation and condensation pressure drop than pure R134a. Torikoshi and Kawabata (1992) confirmed the increase in evaporation pressure drop with the addition of lubricant; however, they found that lubricant had no significant effect on the condensation pressure drop.

The quest for the development of a model to predict two-phase pressure drop of refrigerant mixtures has been met with limited success. Signal et al. (1983) used the Martinelli-Nelson (1948) correlation to predict the pressure drop during forced convection boiling of pure R12 and four different mixtures of R13 and R12. Signal et al. (1983) developed a pressure drop model for pure fluids and a pressure drop model for mixtures where the maximum percent deviation between the model and the measured pressure drop was within $\pm 30 \%$. Jung and Radermacher (1989) measured the pressure drop during horizontal flow boiling of pure R22, R114, R12, and R152a and several mixtures of these refrigerants. They correlated their pressure drop 
measurements to an average residual of $8.4 \%$ by applying a simple thermodynamic corresponding states principle.

Models to predict two-phase pressure drop of refrigerant/lubricant mixtures with similar limited success have been developed. Tichy et al. (1986) presented an experimental investigation of pressure drop in forced-convection condensation and evaporation of R12/lubricant mixtures. For condensation, Tichy et al. (1986) modified the Lockhart-Martinelli (1949) relation for frictional pressure drop and a homogeneous void fraction model. For evaporation, Tichy et al. (1986) modified the Dukler II (1964) frictional pressure-drop correlation and the homogeneous void fraction model. Overall, the evaporation and condensation models predicted $85 \%$ of the data to within $\pm 25 \%$. Tichy et al. (1986) found that, because of a change in liquid properties as compared to the pure fluid, the presence of lubricant increased the pressure drop for condensation and evaporation. The effect of lubricant on the pressure drop was significantly more pronounced for evaporation than it was for condensation. Tichy et al. (1986) also argued that the lubricant encouraged additional wall wetting, which acted to increase the pressure drop.

Presently, the literature does not provide the heat exchanger designer with a satisfactory pressure drop correlation for evaporation and condensation within smooth and micro-fin tubes with alternative refrigerants with and without lubricants. This paper presents a pressure drop correlation for all of these conditions. In an effort to meet this need, the correlation was developed from a database consisting of the following pure and mixed refrigerants: R125, R134a, R32, R410A (R32/125 50/50 \% mass), R22, R407C (R32/125/134a, 23/25/52 \% mass) and R32/134a (25/75 \% mass). Mixtures of R12, R22, and R134a with various lubricants were also correlated with the new pressure drop model.

\section{EXPERIMENTS}

Figure 1 shows a schematic of the experimental apparatus used to establish and measure heat transfer and pressure drop in a micro-fin tube. The local convective boiling heat-transfer coefficients were reported in Kaul et al. (1996). Kedzierski and Goncalves (1999) reported the local condensation heat-transfer coefficients and pressure drops. The condensation and 


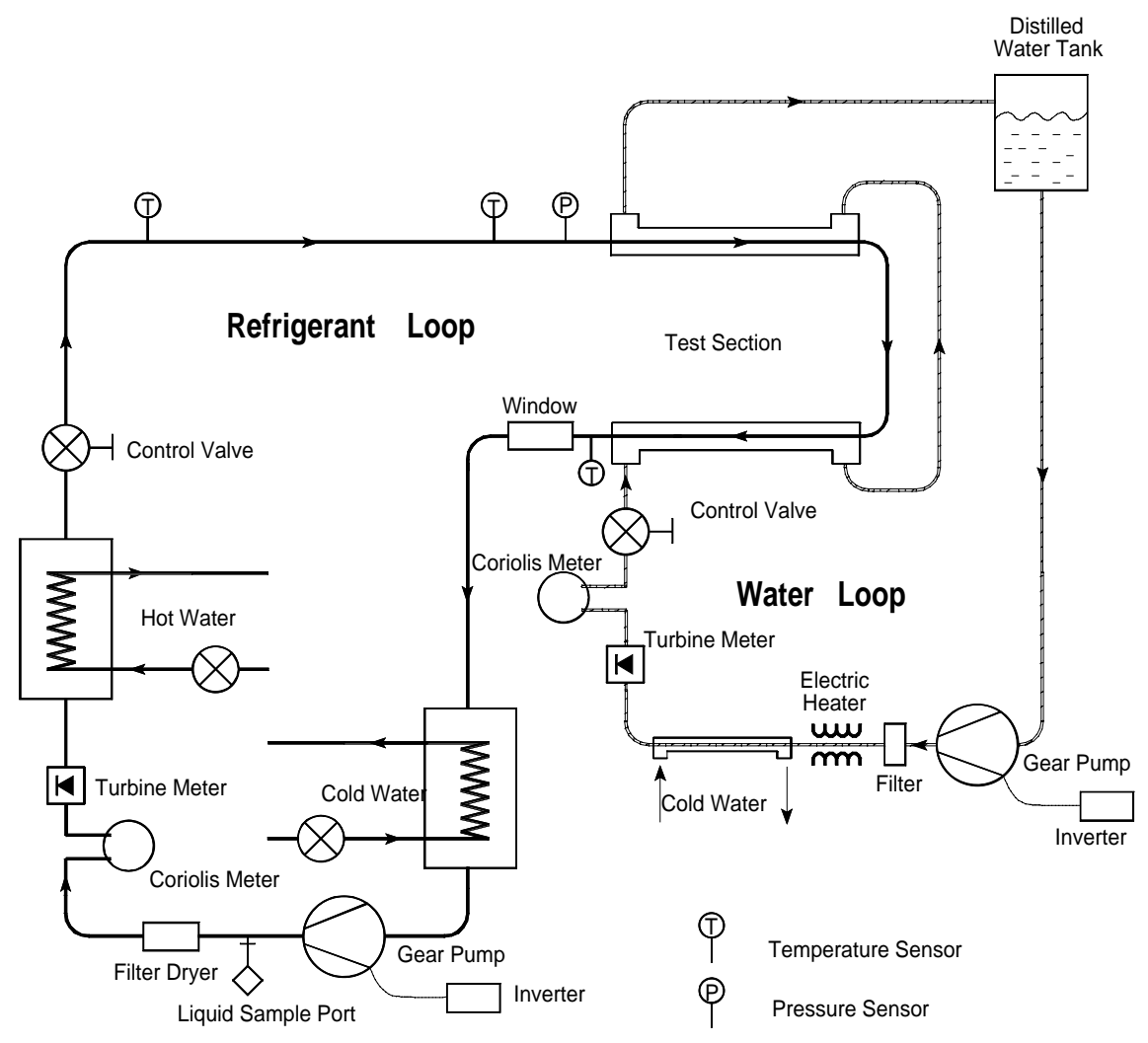

Figure 1. Schematic diagram of experimental apparatus

the evaporative pressure drops from these studies are given in Appendix A and Appendix B, respectively. The refrigerant flow rate, pressure, and single phase state were fixed at the inlet to the test section. The water flow rate and the inlet temperature were fixed to establish the overall refrigerant quality change in the test section. The differential pressure drop was measured over four elements of the test section. Only the two center test section elements were used to develop the pressure drop correlation. This was done to ensure that only saturated two-phase pressure drop data was analyzed. The test section consisted of a pair of $3.34 \mathrm{~m}$ long, horizontal tubes connected by a U-bend. A subcooler before the pump and an evaporator after the pump balanced the heat load of the refrigerant side and maintained a fixed test pressure. A magnetically coupled gear pump delivered the test refrigerant to the entrance of the test section as superheated vapor or subcooled liquid for condensation and evaporation tests, respectively. Another magnetically coupled gear pump was used to maintain the water flow rate. The temperature of the water loop was maintained with a water-chilled heat exchanger and variable electric heaters. The refrigerant 


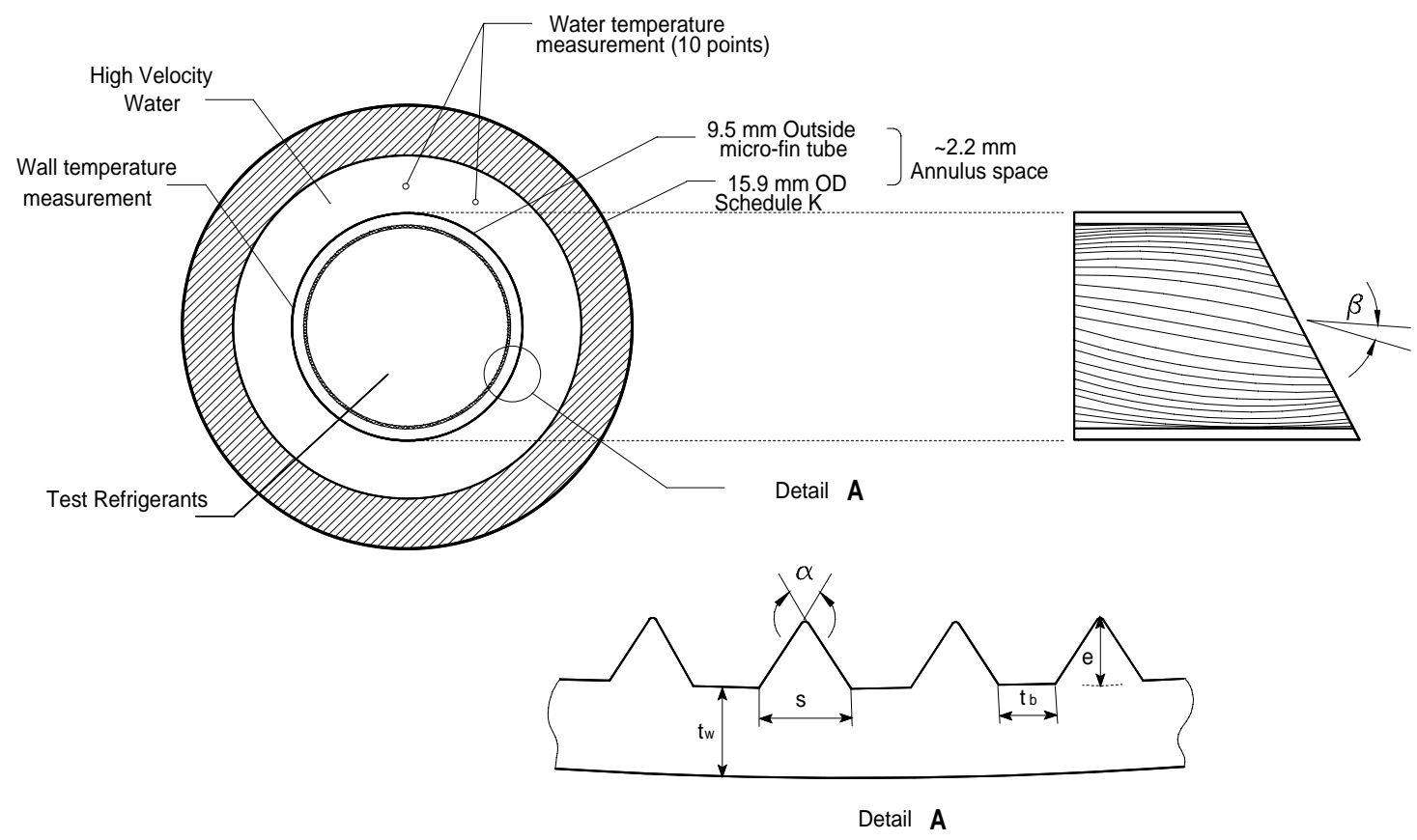

Figure 2. Cross-section of test section.

and water flow rates were controlled by varying the pump speeds with frequency inverters. Redundant flow rate measurements were made with Coriolis flowmeters and turbine flowmeters for both the refrigerant and water sides.

The standard uncertainty is the positive square root of the estimated variance. The individual standard uncertainties are combined to obtain the combined standard uncertainty by the law of propagation of uncertainty. The standard uncertainty becomes an expanded uncertainty when it is multiplied by a coverage factor to correspond to a particular confidence interval. All of the measurement uncertainties reported in this document are expanded uncertainties for a $95 \%$ confidence interval.

Figure 2 shows a cross section of the test section. Table 1 gives the geometric parameters of the copper Turbo-A micro-fin tube that was used in this study. The test refrigerant flowed inside the micro-fin tube while distilled water flowed in the annulus that surrounded the micro-fin tube either in counterflow or in parallel flow to the refrigerant. The annulus gap was $2.2 \mathrm{~mm}$, and the micro-fin tube wall thickness was $0.3 \mathrm{~mm}$. The micro-fin tube had sixty $0.2 \mathrm{~mm}$ high fins with 
Table 1. Geometric parameters of Turbo-A micro-fin tube.

\begin{tabular}{l|l}
\hline \multicolumn{1}{c|}{ Parameters } & Dimension \\
\hline$D_{o}$ & $9.52 \mathrm{~mm}$ \\
$t_{w}$ & $0.3 \mathrm{~mm}$ \\
$D_{r}$ & $8.92 \mathrm{~mm}$ \\
$A_{c}$ & $60.8 \mathrm{~mm}^{2}$ \\
$e$ & $0.2 \mathrm{~mm}$ \\
$P$ & $0.47 \mathrm{~mm}$ \\
$\beta$ & $18^{\circ}$ \\
$\alpha$ & $50^{\circ}$ \\
$N$ & 60 \\
$S_{p}$ & $0.707 \mathrm{~mm}$ \\
$D_{h}$ & $5.45 \mathrm{~mm}$ \\
Distance between pressure taps & $1.587 \mathrm{~m}, 1.588 \mathrm{~m}$
\end{tabular}

$18^{\circ}$ helix angle. For this geometry, the cross sectional flow area $\left(A_{c}\right)$ was $60.8 \mathrm{~mm}^{2}$ giving an equivalent smooth diameter $\left(D_{e}\right)$ of $8.8 \mathrm{~mm}$. The root diameter of the micro-fin tube was 8.91 $\mathrm{mm}$. The inside-surface area per axial length of the tube was estimated to be $44.6 \mathrm{~mm}$. The hydraulic diameter $\left(D_{h}\right)$ of the micro-fin tube was estimated from Eq. (1) (see p. 13) to be 5.45 $\mathrm{mm}$. The ratio of the inner surface area of the micro-fin tube to the surface area of a smooth tube of the same $D_{e}$ is 1.6 . The fins rifle down the axis of the tube at a helix angle of $18^{\circ}$ with respect to the tube axis.

Figure 3 provides a detailed description of the test section. The annulus was constructed by connecting a series of tubes with 14 pairs of stainless steel flanges. This construction permitted the measurement of both the outer micro-fin wall temperature and the water temperature change as discussed in the following two paragraphs.

As shown in Figure 3, six refrigerant pressure taps along the test section allowed the measurement of the upstream absolute pressure and five pressure drops along the test section. Two sets of two water pressure taps were used to measure the water pressure drop along each tube. Differential pressure transducers with an expanded uncertainty of $1 \%$ of the measurement were used to measure the pressure drops. Also, a sheathed thermocouple measured the refrigerant temperature at each end of the two refrigerant tubes with the junction of each located 


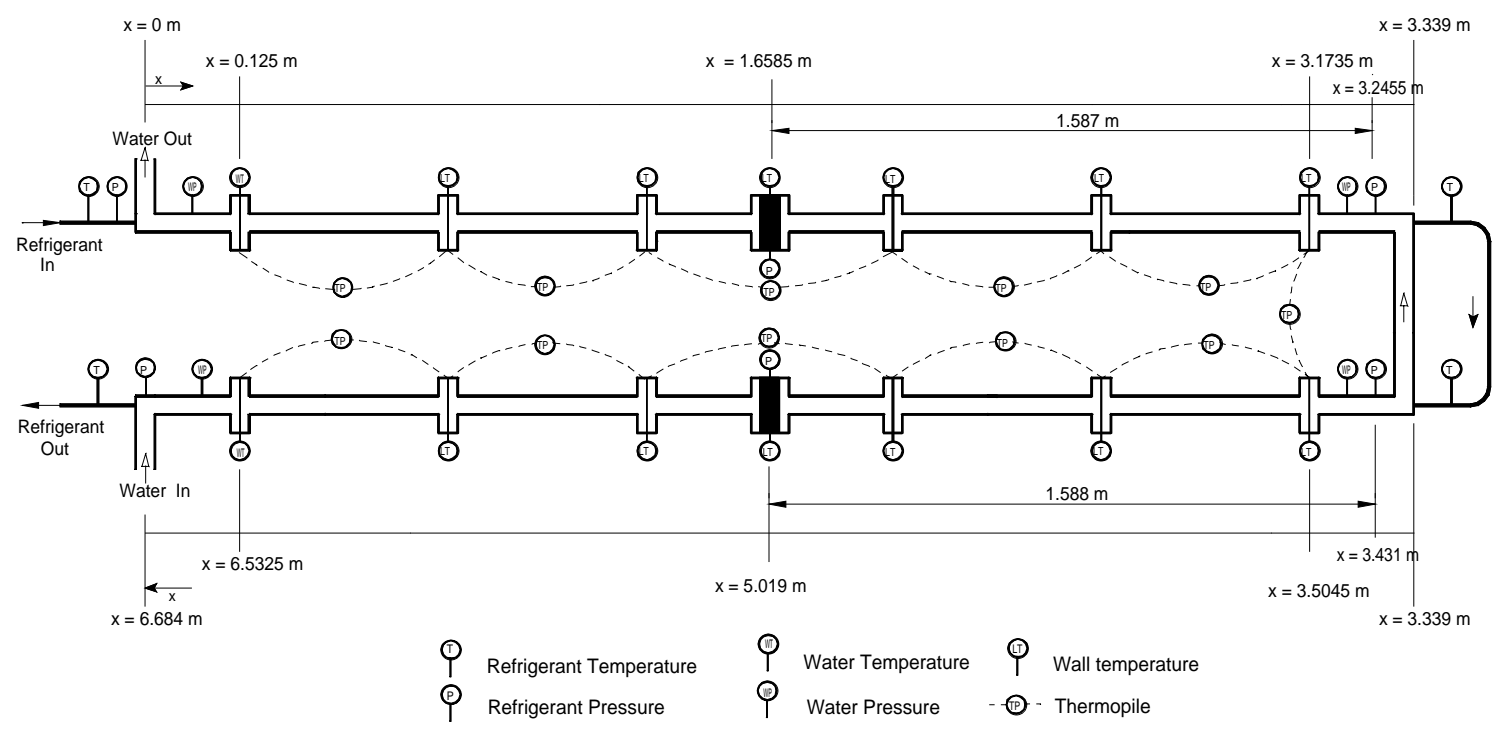

Figure 3. Detail schematic of test section.

at the center radially. Only the thermocouple at the inlet of the first tube was used in the calculations. The entire test section was wrapped with $5 \mathrm{~cm}$ of foam insulation to minimize heat transfer between the water and the ambient. The pressure drops used in this paper were measured between $\mathrm{x}=1.6585 \mathrm{~m}$ and $\mathrm{x}=3.2456 \mathrm{~m}$, and between $\mathrm{x}=3.4310 \mathrm{~m}$ and $\mathrm{x}=5.0190 \mathrm{~m}$, which the tube lengths are $1.5870 \mathrm{~m}$, and $1.5880 \mathrm{~m}$, respectively. The thermodynamic quality was calculated with an energy balance to an expanded uncertainty of $\pm 8 \%$.

Table 2 shows the test conditions. More than 800 pressure drop measurements were taken for various mass fluxes and various pressures. The mass flux was varied from $88 \mathrm{~kg} / \mathrm{m}^{2} \mathrm{~s}$ to 469 $\mathrm{kg} / \mathrm{m}^{2} \mathrm{~s}$ and from $306 \mathrm{~kg} / \mathrm{m}^{2} \mathrm{~s}$ to $367 \mathrm{~kg} / \mathrm{m}^{2} \mathrm{~s}$ for the condensation and the evaporation data, respectively. The expanded uncertainty of the mass flux measurement was $\pm 2 \%$. The absolute pressure was varied from $1.1 \mathrm{MPa}$ to $2.0 \mathrm{MPa}$ and from $0.36 \mathrm{MPa}$ to $0.95 \mathrm{MPa}$ for the condensation and for the evaporation data, respectively. The expanded uncertainty in the absolute pressure measurement was $\pm 1.5 \%$ of the measurement. The inlet quality was varied from $100 \%$ to $16.3 \%$, and the outlet quality was varied from $87.3 \%$ to $6.4 \%$ for condensation. The inlet quality was varied from $2.9 \%$ to $48.5 \%$, and the outlet quality was varied from $9.2 \%$ to $63.9 \%$ for evaporation. 
Table 2. Summary of test conditions.

\begin{tabular}{l|l|c|c|c|c}
\multicolumn{2}{l|}{} & $\begin{array}{c}\text { Mass } \\
\text { Flux } \\
\left(\mathrm{kg} / \mathrm{m}^{2} \mathrm{~s}\right)\end{array}$ & $\begin{array}{c}\text { Pressure } \\
(\mathrm{MPa})\end{array}$ & $\begin{array}{c}\text { Quality } \\
\text { at inlet } \\
(\%)\end{array}$ & $\begin{array}{c}\text { Quality } \\
\text { at outlet } \\
(\%)\end{array}$ \\
\hline \multirow{5}{*}{ Condensation } & R32 & $88-335$ & 2.0 & $19.1-100$ & $8.6-83.4$ \\
& R125 & $194-468$ & 1.7 & $21.4-100$ & $10.0-87.3$ \\
& R410A & $138-468$ & 1.9 & $16.3-100$ & $6.4-86.0$ \\
& R134a & $120-469$ & 1.1 & $19.0-100$ & $8.8-83.9$ \\
\hline \multirow{5}{*}{ Evaporation } & R32 & $312-364$ & 0.95 & $2.9-38.4$ & $9.2-51.6$ \\
& R125 & $316-367$ & 0.78 & $3.7-47.9$ & $12.9-60.5$ \\
& R410A & $313-364$ & 0.92 & $2.9-36.7$ & $9.5-51.7$ \\
& R134a & $306-364$ & 0.36 & $3.4-16.3$ & $36.2-50.4$ \\
& R22 & $311-370$ & 0.59 & $3.6-41.7$ & $11.7-59.1$ \\
& R407C & $311-370$ & 0.61 & $6.7-48.5$ & $16.8-63.9$ \\
& R32/R134a & $310-364$ & 0.55 & $5.4-38.0$ & $13.4-52.0$
\end{tabular}

\section{REFRIGERANT PROPERTY EVALUATION}

All of the thermodynamic and transport properties for both pure and mixed refrigerants that were used in the data analysis and pressure drop predictions were calculated using property routines from REFPROP 4.01 (Gallagher, 1993). The thermodynamic mixture properties were calculated with the Extended Corresponding States (ECS). The high accuracy Modified Benedict-Webb-Rubin (MBWR) equation of state was selected for pure fluids, R32, R125, and R134a.

\section{COMPARISON OF DATA TO EXISTING CORRELATIONS}

A total of 831 data points from the NIST test apparatus were analyzed in the present study: 205 for condensation and 626 for evaporation. These measurements were compared to two homogeneous models and two separated models. The homogenous model, also known as 'the friction factor' or 'fog flow' model, considers the two phases to flow as a single phase possessing mean fluid properties. The separated flow model considers the phases to be segregated into a liquid and a vapor stream. The above models were developed from smooth tube data. In the above correlations, the hydraulic diameter of the micro-fin tube was used in lieu of the smooth tube diameter. 
In the present analysis, the hydraulic diameter $\left(D_{h}\right)$ was used to correlate the pressure drop data. Kedzierski and Goncalves (1999) recommend that the hydraulic diameter for micro-fin tubes be calculated as:

$$
D_{h}=\frac{4 A_{c} \cos \beta}{N S_{p}}
$$

where $S_{p}$ is the perimeter of one fin and channel taken perpendicular to the axis of the fin, $N$ is the number of fins, $A_{c}$ is the cross-sectional flow area, $A_{c}=\frac{\pi D_{o}^{2}}{4}-N A_{f}$, and $\beta$ is the helix angle of the fin. Figure 4 illustrates the various fin parameters that were used in the calculation of $D_{h}$.

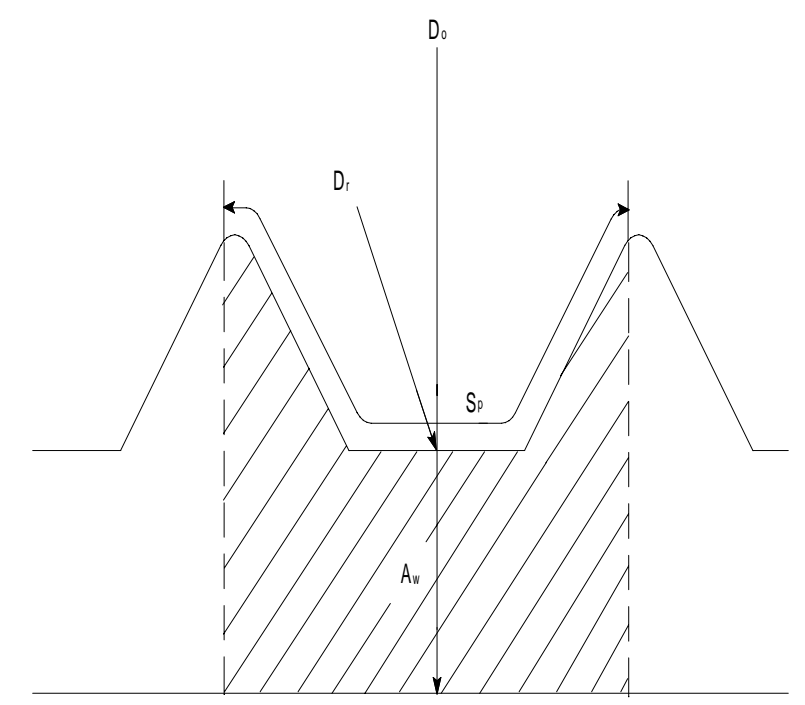

Figure 4. Detail cross section of micro-fin tube.

\section{Homogenous Flow Models}

In 1964, Bo Pierre published a paper on the prediction of the evaporation pressure drop for R12 and R12/lubricant mixtures in $12 \mathrm{~mm}$ and $18 \mathrm{~mm}$ diameter, horizontal, smooth tubes. Although many pressure drop correlations have been developed since 1964, the Bo Pierre (1964) correlation remains appealing because of its simplicity and validity:

$$
\Delta P_{B P}=\left[f_{B P}+\frac{\left(x_{\text {out }}-x_{\text {in }}\right) D}{\bar{x} L}\right] \frac{G^{2} \bar{x} v_{g} L}{D}
$$


where $\bar{x}$ is the linearly averaged thermodynamic quality over the length $(L)$. The specific volume of the vapor $\left(v_{g}\right)$, the mass velocity $(G)$, and the properties for the two-phase friction factor $\left(f_{B P}\right)$ are evaluated at the linear-average refrigerant temperature. The two-phase friction factor for pure refrigerant, valid for $\left(\operatorname{Re} / K_{f}\right)>1$, is given as:

$$
f_{B P}=0.0185\left(\frac{\mathrm{K}_{f}}{\mathrm{Re}}\right)^{0.25}
$$

where Pierre's boiling number is defined as: $\mathrm{K}_{f}=\frac{\Delta x h_{f g}}{L g}$

Pierre's friction factor for the case where lubricant is present is given in the following equation which is valid for $\left(\operatorname{Re} / \mathrm{K}_{f}\right)>2$ :

$$
f_{B P}=0.053\left(\frac{\mathrm{K}_{f}}{\mathrm{Re}}\right)^{0.25}
$$

Figure 5 compares both condensation and evaporation pressure drop data to the predictions obtained from Eq. (2). Overall, the Bo Pierre correlation predicted the measured data with an average residual of $15.8 \%$. However, a few of the low pressure drop data were underpredicted by $50 \%$ to $60 \%$. The ability of the Pierre (1964) pressure drop correlation to predict the condensation pressure drop data is adequate considering that it was developed with only evaporation pressure drop data.

The homogeneous pressure drop model as given by Collier and Thome (1994) is:

$$
\Delta P_{C}=\left(\frac{2 f_{B} G^{2} v_{f} L}{D}\right) \cdot\left[1+\bar{x}\left(\frac{v_{f g}}{v_{f}}\right)\right] \cdot\left[1+\bar{x}\left(\frac{\mu_{f g}}{\mu_{g}}\right)\right]^{-1 / 4}+G^{2} v_{f}\left(\frac{v_{f g}}{v_{g}}\right)\left(x_{o}-x_{i}\right)
$$

In this model the two-phase frictional pressure drop is expressed in terms of the single phase pressure drop for the total flow considered as liquid. The two-phase friction factor is calculated from the Blasius (1913) equation:

$$
f_{B}=\frac{0.079}{\operatorname{Re}_{f o}^{0.25}}
$$




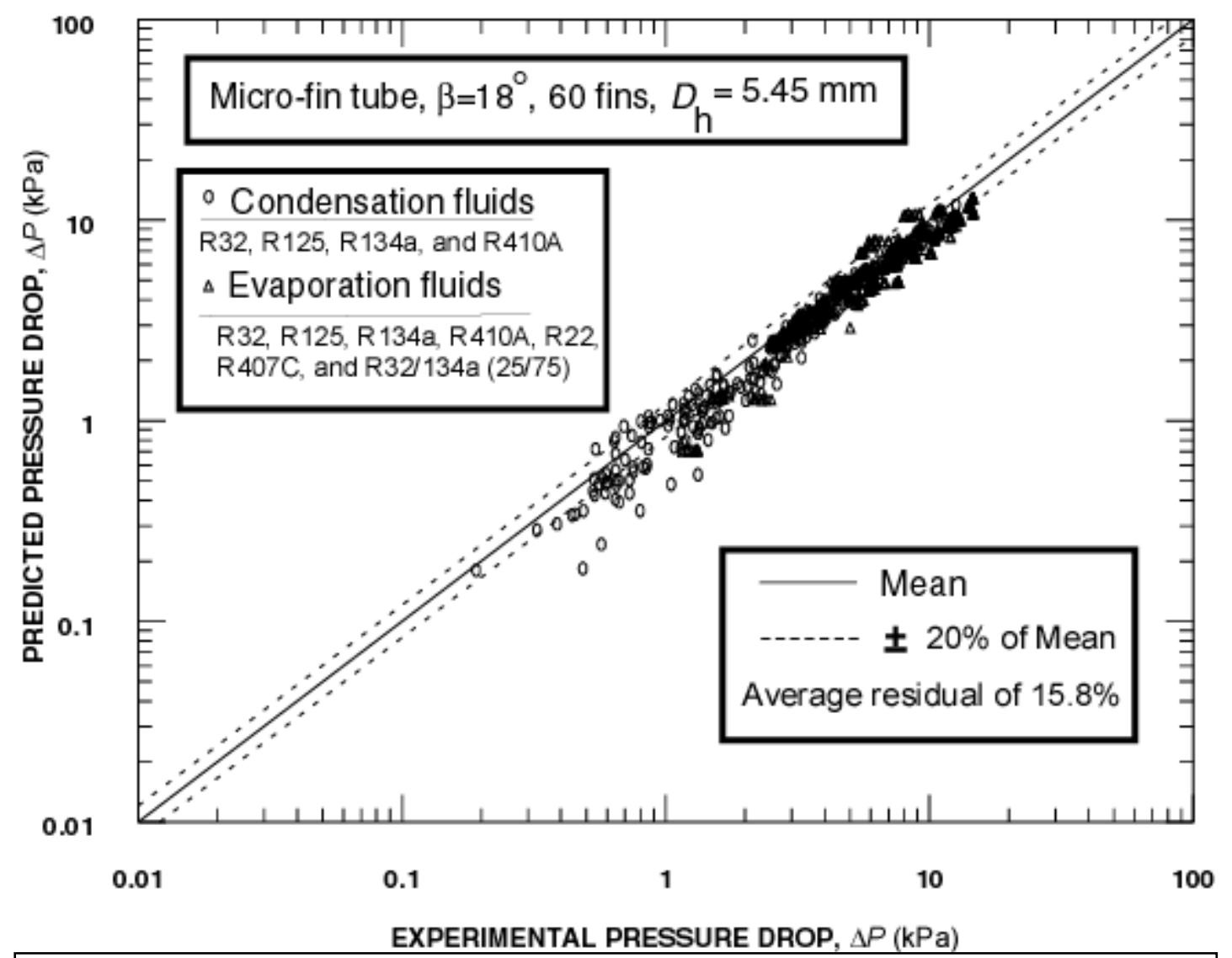

Figure 5 Prediction of NIST pressure drop measurements with Pierre (1964) correlation.

Figure 6 compares both condensation and evaporation pressure drop data to the predictions obtained from the homogeneous model given in Collier and Thome (1994). Overall, the homogeneous model underpredicted the measured data on average by $31.4 \%$ for all pressure drop ranges. The underprediction increased for the higher pressure drops. 


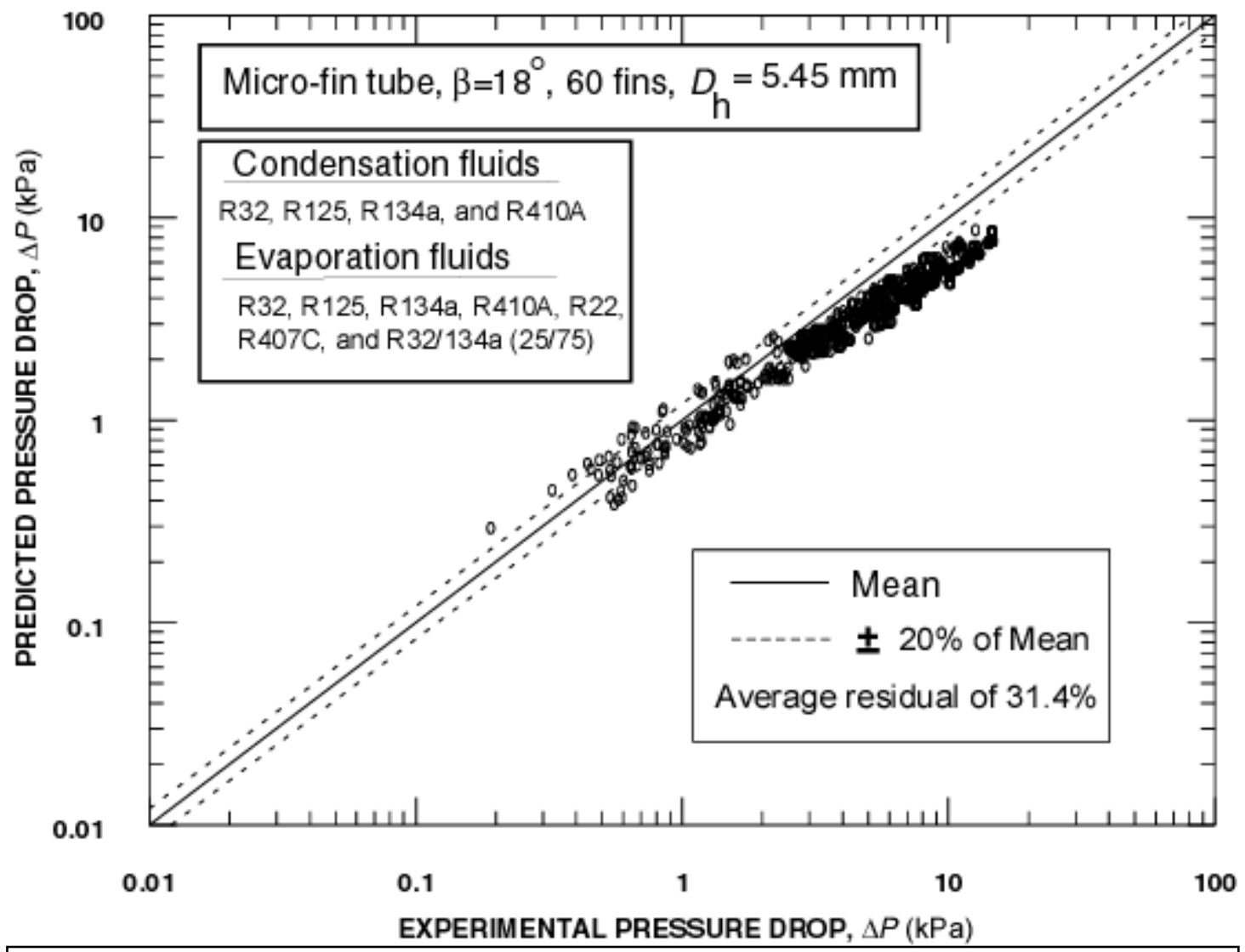

Figure 6 Predictions of NIST pressure drop measurements by homogenous model as given in Collier and Thome (1994).

\section{Separated Flow Models}

The total pressure gradient for horizontal homogeneous and separated flow is represented by the sum of the frictional and momentum components of the pressure gradient (Collier and Thome, 1994):

$$
\begin{aligned}
& \Delta P_{t p}=\Delta P_{F}+\Delta P_{A} \\
& =\left[\frac{2 f_{f} G^{2}(1-x)^{2} v_{f} L}{D}\right] \phi_{f}^{2}+G^{2}\left(x_{o}-x_{i}\right)\left[\left\{\frac{2 x v_{g}}{\alpha}-\frac{2(1-x) v_{f}}{(1-\alpha)}\right\}+\left(\frac{\mathrm{d} \alpha}{\mathrm{d} x}\right)_{p}\left\{\frac{(1-x)^{2} v_{f}}{(1-\alpha)^{2}}-\frac{x^{2} v_{g}}{\alpha^{2}}\right\}\right]
\end{aligned}
$$

Pressure drop correlations based on the separated flow model has been continuously developed since Lockhart and Martinelli (1949) published their classic paper on two-phase gas-liquid flow. 
Lockhart and Martinelli (1949) defined the friction factor as:

$$
f_{f}=\frac{0.045}{\operatorname{Re}_{f}^{0.2}}
$$

The two-phase friction multipliers, $\phi_{l}^{2}$ and $\phi_{g}^{2}$, can be represented as a function of the LockhartMartinelli parameter, $X_{t t}$ :

$$
X_{t t}=\left(\frac{1-x}{x}\right)^{0.9}\left(\frac{\mu_{g}}{\mu_{f}}\right)^{0.1}\left(\frac{v_{f}}{v_{g}}\right)^{0.5}
$$

Chishom (1968) proposed a modification to the Lockhart-Martinelli correlation by allowing the two-phase frictional multiplier to vary with mass flux.

$$
\phi_{f}^{2}=1+\frac{C}{X_{t t}}+\frac{1}{X_{t t}^{2}}
$$

where $C=\left[\lambda+\left(C_{2}-\lambda\right)\left(v_{f g} / v_{g}\right)^{0.5}\right] \cdot\left[\left(v_{g} / v_{f}\right)^{0.5}+\left(v_{f} / v_{g}\right)^{0.5}\right]$ and $\lambda=0.5 \cdot\left(2^{(2-n)}-2\right)$. For rough tubes $(n$ $=0$ in Blasius equation) $\lambda=1$; for smooth tubes $(n=0.25) \lambda=0.68$. At the critical pressure $\left(v_{f}=v_{g} ; \quad v_{f g}=0\right) \quad C$ takes on a value of 2 for rough tubes and 1.36 for smooth tubes. For mass fluxes less than $2000 \mathrm{~kg} / \mathrm{m}^{2} \mathrm{~s}, C_{2}$ and $\lambda$ are $2000 / G$ and 0.75 (corresponding to $n=0.2$ ) for smooth tubes; and $1500 / G$ and 1 (corresponding to $n=0$ ) for rough tubes.

Butterworth (1975) reduced six void fraction models into one equation with variable constants:

$$
\alpha=\frac{1}{1+0.28 \cdot\left(\frac{1-x}{x}\right)^{0.64}\left(\frac{\rho_{g}}{\rho_{f}}\right)^{0.36}\left(\frac{\mu_{f}}{\mu_{g}}\right)^{0.07}}
$$

Figure 7 compares the measured evaporation and condensation pressure drop to the pressure drop predictions by the separated flow model given by Eq. 7. The frictional pressure drop was calculated by the Lockhart-Martinelli correlation using the two-phase frictional multiplier proposed by Chishom. The separated flow model overpredicted the measured data on average by $31.1 \%$ for all pressure drop ranges. Specifically, overpredictions of over $100 \%$ were evident 
for the small pressure drop range. For the larger pressure drops, some data were overpredicted by more than $20 \%$.

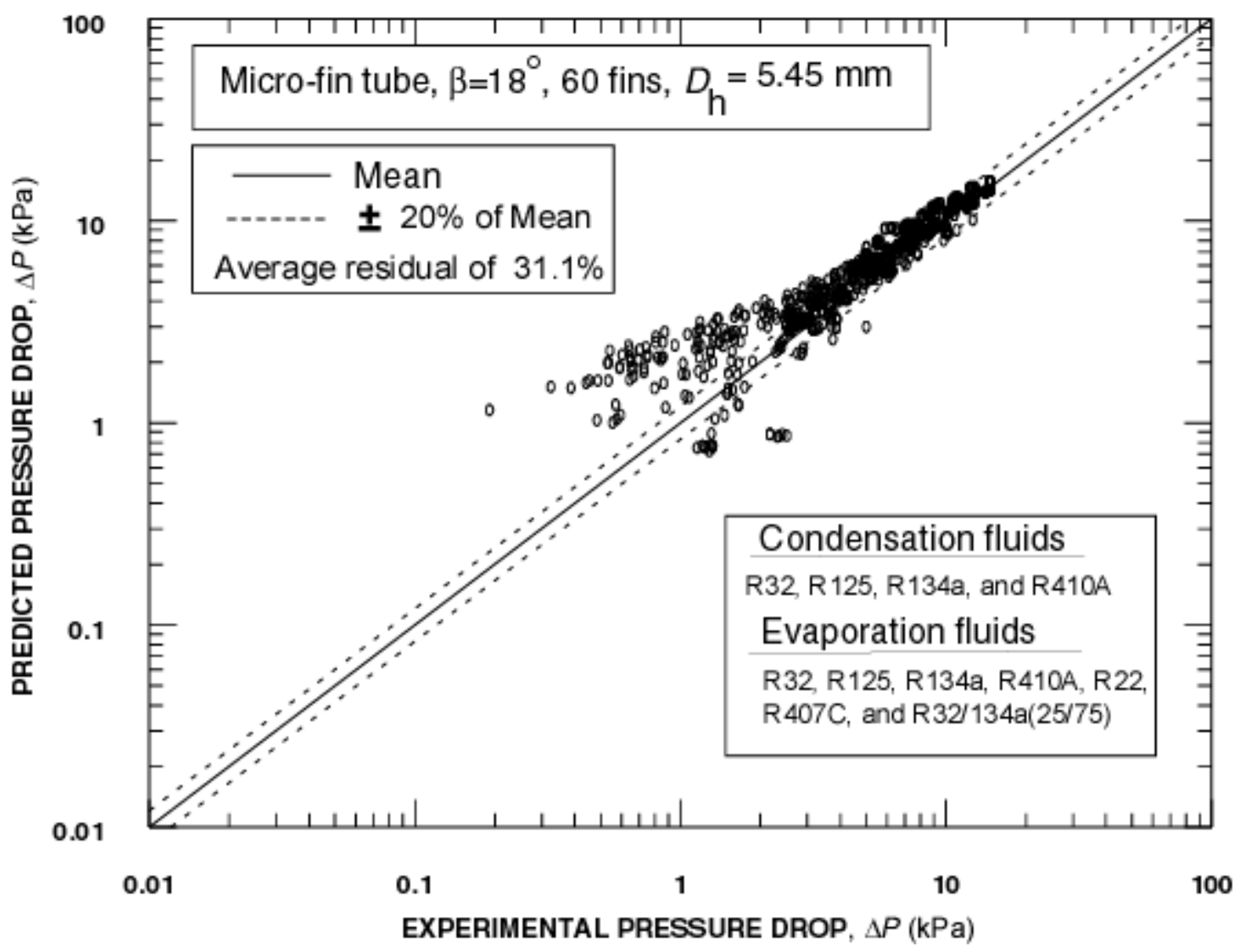

Figure 7. Prediction of NIST pressure drop measurements by separated flow model (Eq. 7)

Starting with the Martinelli-Nelson correlation (1948), Jung and Radermacher (1989) developed a new correlation from flow boiling pressure drop data for pure and mixed refrigerants with a wide absolute pressure range $(0.2 \mathrm{MPa}$ to $0.8 \mathrm{MPa})$. They used a regression analysis to remove the explicit dependence of the two-phase multiplier on $X_{t t}$ :

$$
\phi_{t p}^{2}=30.78 x^{1.323}(1-x)^{0.477} P_{r}^{-0.7232}
$$




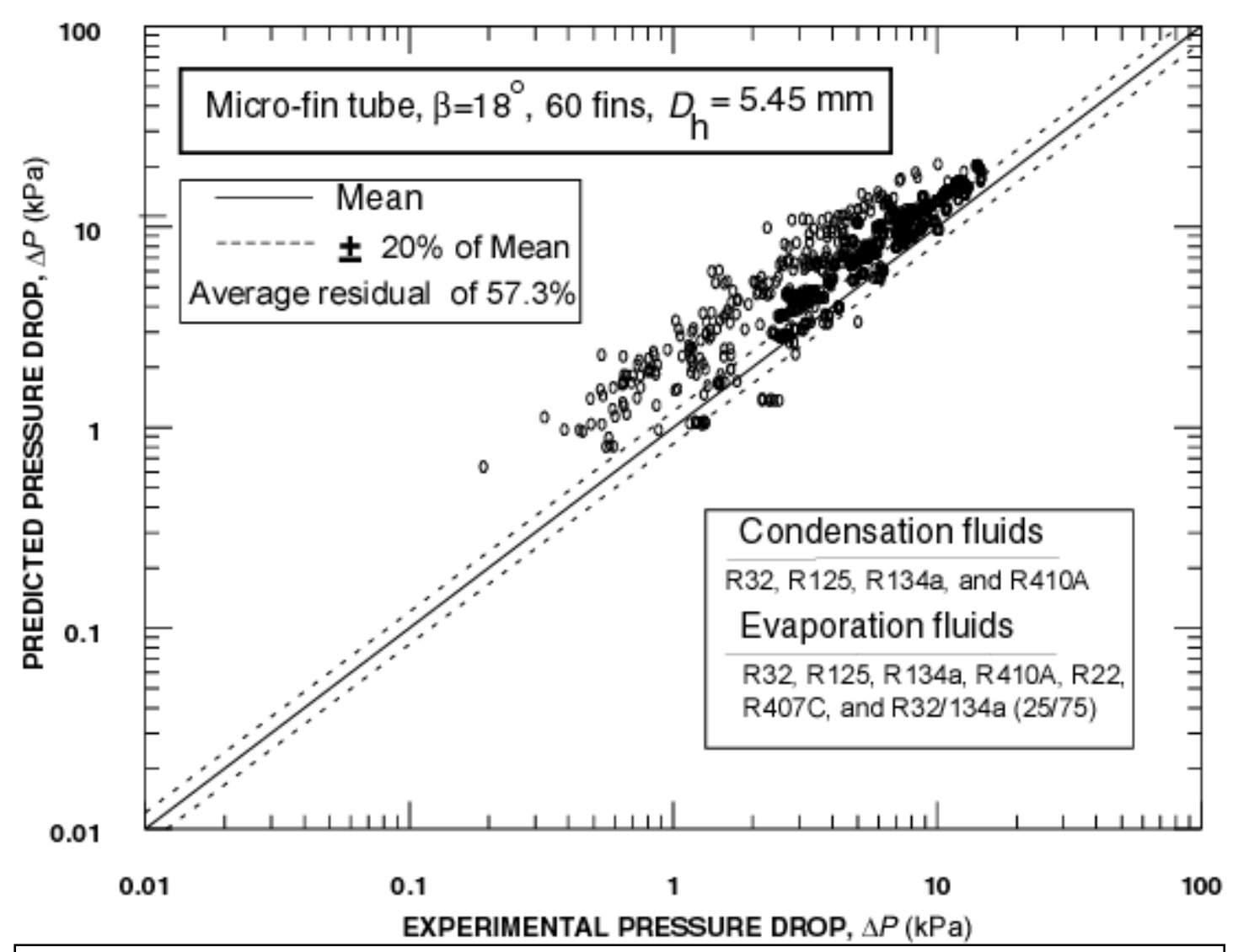

Figure 8 Predictions of NIST pressure drop measurements with Jung-Radermacher (1989) correlation

The Jung and Radermacher (1989) correlation for the two-phase pressure drop is:

$$
\Delta P_{t p}=\frac{2 f_{f o} G^{2} L v_{f}}{D}\left[\frac{1}{\Delta x} \int_{x_{i n}}^{x_{\text {out }}} \phi_{t p}^{2} \mathrm{~d} x\right]
$$

The integral of Eq. (13) is evaluated numerically by subtracting the integral between $0 \%$ to the inlet vapor quality, $x_{i n}$, from the integral between $0 \%$ and the outlet vapor quality, $x_{\text {out }}$. The friction factor is calculated by the following equation:

$$
f_{f o}=0.046 \mathrm{Re}^{-0.2}
$$

Figure 8 compares the predictions from the Jung-Radermacher (1989) pressure drop correlation to the NIST micro-fin pressure drop measurements. Averaged over all pressure ranges and refrigerants, the Jung-Radermacher correlation overpredicted the measured data by $57.3 \%$.

The Jung-Radermacher correlation was obtained from convective boiling data in a smooth tube. The hydraulic diameter concept can effectively be used to redistribute the wall shear from a circular to a noncircular tube for equivalent mixing at the wall. However, the micro-fins - 
because of their size - are expected to increase a roughness by providing an additional mixing effect at the wall that cannot be accounted for by an extrapolation of a smooth tube pressure drop correlation with the hydraulic diameter concept. Consequently, it was surprising that the correlation overpredicted rather than underpredicted the pressure drop in micro-fin tube.

\section{THE NEW CORRELATION FOR PRESSURE DROP}

\section{Pure Refrigerants}

From among the four correlations presented in the above analysis, the predictions of the Pierre (1964) correlation were closest to the NIST micro-fin pressure drop data for convective evaporation and condensation. However, as shown in Figure 5, these predictions are not centered about the mean of the data. Consequently, a new two-phase friction factor $\left(f_{N}\right)$ for Pierre (1964) pressure drop model was correlated to the NIST two-phase, micro-fin pressure drop data. The Pierre (1964) correlation was further modified by using the hydraulic diameter $\left(D_{h}\right)$ and by including the specific volume of liquid in the calculation of the average specific volume of the two-phase fluid. (Pierre neglected the specific volume of liquid). The modified correlation becomes :

$$
\Delta P_{t p}=\Delta P_{\text {friction }}+\Delta P_{\text {accelation }}=\left(\frac{f_{N} L\left(v_{\text {out }}+v_{\text {in }}\right)}{D_{h}}+\left(v_{\text {out }}-v_{\text {in }}\right)\right) G^{2}
$$

The uncertainty of the fit was reduced when the liquid specific volume was included. The specific volume of the two-phase fluid ( $v$ ) was obtained from a linear quality weighted sum of the vapor and liquid specific volumes at either the outlet or inlet of the segment $\mathrm{L}$. The total mass velocity $(G)$, and the properties for the two-phase friction factor $\left(f_{N}\right)$ were evaluated at a linearly averaged refrigerant temperature. The new two-phase friction factor is:

$$
f_{N}=0.00506 \operatorname{Re}_{f o}{ }^{-0.0951} \mathrm{~K}_{f}^{0.1554}
$$

The friction factor is based on the all liquid Reynolds number, $\operatorname{Re}_{f o}=\frac{G D_{h}}{\mu_{f}}$, and the two-phase number, $\mathrm{K}_{f}=\frac{\Delta x h_{f g}}{L g}$. The procedure that was used to identify and possibly remove data that had high influence and/or high leverage on the regression using the hat matrix and Cook's distance (Hamilton, 1992) is described by Kedzierski and Goncalves (1997). The friction factor 
correlation that was obtained by Kedzierski and Goncalves (1999) for condensation alone was on average and consistently $5 \%$ less than that given by Eq. (16) for $\operatorname{Re}_{f o}$ between 5000 and 25000 .

Figure 9 shows improvement obtained with the modification of the Pierre (1964) pressure drop model. The NIST measured pressure drop data is predicted with an average residual of $10.8 \%$ for all pressure drop ranges and refrigerants.

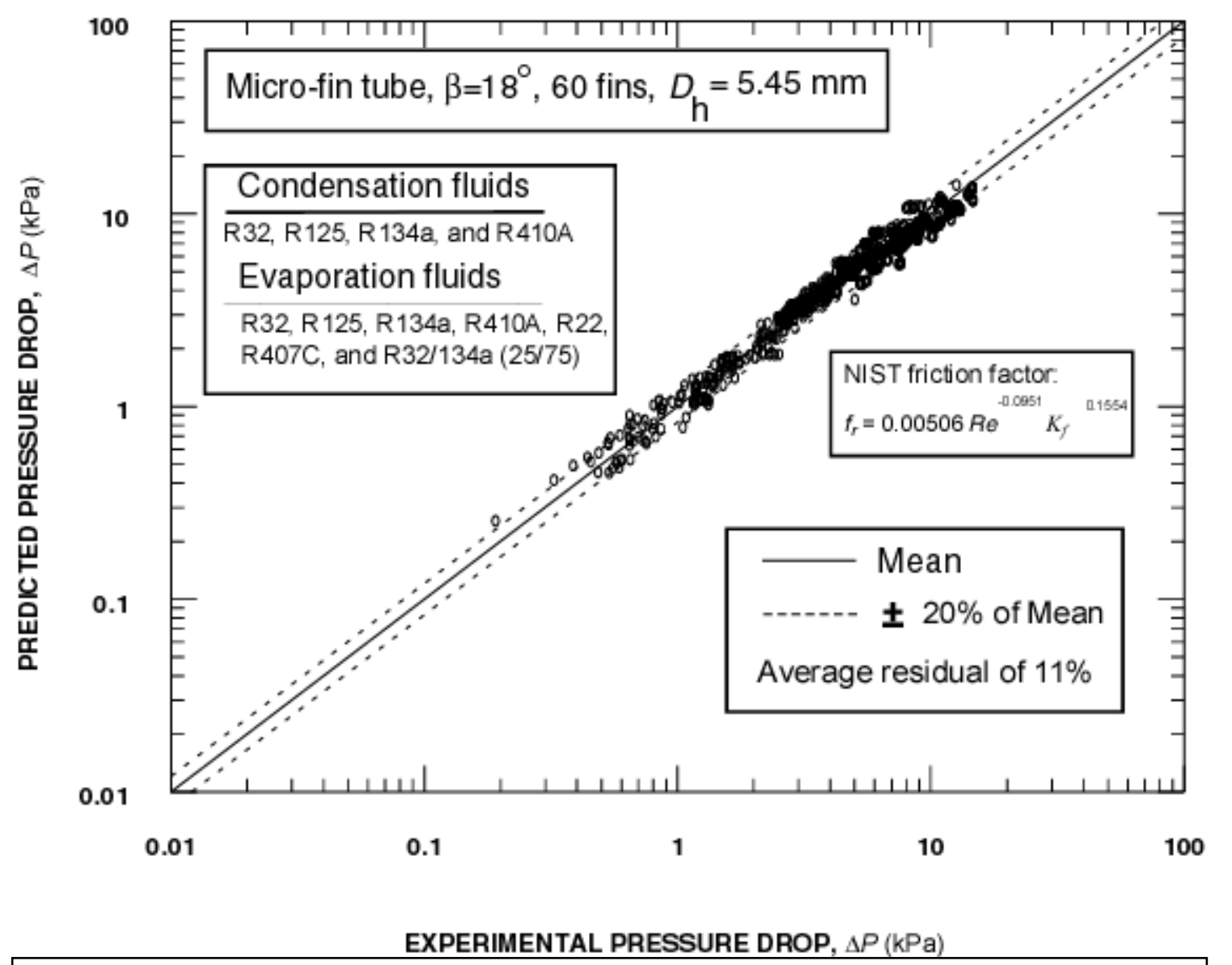

Figure 9 Predictions of NIST pressure drop measurements with NIST correlation.

The new correlation was verified with pressure drop data from Eckels et al. (1991 and 1993) and Pate et al. (1991), which are given in Appendix C and Appendix D, respectively. Table 3 shows geometric parameters of smooth and micro-fin tubes that were used by both researchers. Table 4 shows the test conditions for each data set. Pate et al. (1991) measured the pressure drop during condensation and evaporation of R12, R134a, and R22. Eckels et al. (1991 and 1993) measured the pressure drop during condensation of R22, R134a, R410A, R502, and R507. The hydraulic 
diameters of the micro-fin tubes were calculate from Eq. (1) and used to correlate both condensation and evaporation data.

Figure 10 compares the predictions of the NIST correlation to the convective condensation pressure drop data of Eckels et al. (1991 and 1993) in smooth tube and three different micro-fin tubes. The smooth-tube and micro-fin-tube data are predicted to within $13.1 \%$ and $17.6 \%$, respectively, for all ranges and refrigerants.

Table 3. Geometric tube parameters of Pate (1991) and Eckels (1991 and 1993).

\begin{tabular}{|c|c|c|c|c|c|c|c|c|}
\hline \multirow{3}{*}{$\begin{array}{l}\text { Researcher } \\
\text { Tube type }\end{array}$} & \multicolumn{4}{|c|}{ Pate et al. } & \multicolumn{4}{|c|}{ Eckels et al. } \\
\hline & \multicolumn{2}{|c|}{ Smooth } & \multicolumn{2}{|c|}{ Micro-fin } & \multirow{2}{*}{$\frac{\text { Smooth }}{\text { (3/8 in) }}$} & \multicolumn{3}{|c|}{ Micro-fin } \\
\hline & (3/8 in) & (1/2 in) & (3/8 in) & (1/2 in) & & (3/8in) & (5/8 in) & (5/16in) \\
\hline$\overline{D_{o}(\mathrm{~mm})}$ & 9.52 & 12.70 & Same & 12.70 & 9.53 & Same & 15.90 & 7.94 \\
\hline$t_{w}(\mathrm{~mm})$ & 0.760 & 0.800 & as & 0.406 & 0.760 & as & 0.508 & 0.305 \\
\hline$D_{r}(\mathrm{~mm})$ & 8.01 & 11.10 & NIST & 11.98 & 8.01 & NIST & 14.68 & 7.33 \\
\hline$A_{c}\left(\mathrm{~mm}^{2}\right)$ & 50.3 & 96.8 & & 108.5 & 50.3 & & 170 & 40.6 \\
\hline$e(\mathrm{~mm})$ & - & - & & 0.254 & - & & 0.305 & 0.203 \\
\hline$P(\mathrm{~mm})$ & - & - & & 0.622 & - & & 0.778 & 0.460 \\
\hline$\beta$ (degrees) & - & - & & 18 & - & & 27 & 18 \\
\hline$N$ & - & - & & 60 & - & & 60 & 50 \\
\hline$S_{p}(\mathrm{~mm})$ & - & - & & 0.947 & - & & 1.196 & 0.712 \\
\hline$D_{h}(\mathrm{~mm})$ & 8.0 & 11.1 & & 7.432 & 8.01 & & 8.443 & 4.339 \\
\hline $\begin{array}{l}\text { Distance between } \\
\text { pressure taps }(\mathrm{m})\end{array}$ & 3.97 & 3.97 & 3.97 & 3.97 & 3.81 & 3.78 & 3.78 & 3.78 \\
\hline
\end{tabular}


Table 4. Summary of test conditions for of Pate (1991) and Eckels (1991and 1993).

\begin{tabular}{l|l|l|l|l|l}
\multicolumn{2}{l|}{ Conditions } & Mass Flux $\left(\mathrm{kg} / \mathrm{m}^{2} \mathrm{~s}\right)$ & Pressure (MPa) & $\begin{array}{l}\text { Inlet } \\
\text { quality (\%) }\end{array}$ & $\begin{array}{l}\text { Outlet } \\
\text { quality (\%) }\end{array}$ \\
\hline Pate et al. & Cond. & $125-400$ & $1.5-1.6$ & $80-88$ & $5-15$ \\
& Evap. & $125-400$ & $0.5-0.6$ & $5-20$ & $80-88$ \\
\hline Eckels et al & Cond. & $120-620$ & $0.9-2.5$ & $48-91$ & $2-46$
\end{tabular}

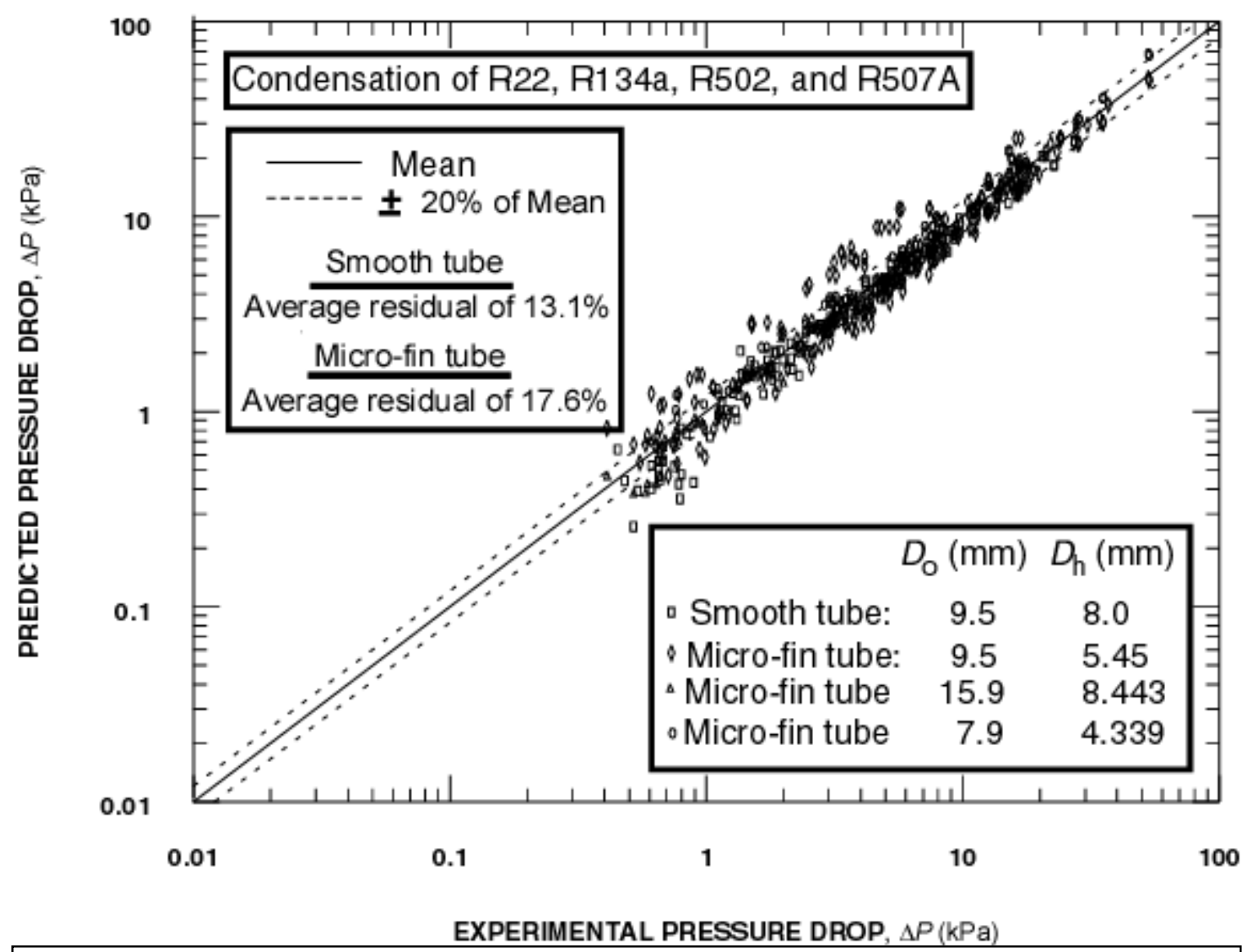

Figure 10 Predictions of Eckels et al. (1991 and 1993) pressure drop measurements with NIST correlation

Figure 11 compares the predictions of the NIST correlation to the convective boiling and condensation pressure drop data of Pate et al. (1991). The present correlation predicts the smooth-tube and micro-fin-pressure drop data of the Pate et al. (1991) to within $15.0 \%$ and 16.1 $\%$, respectively for all ranges and refrigerants. 


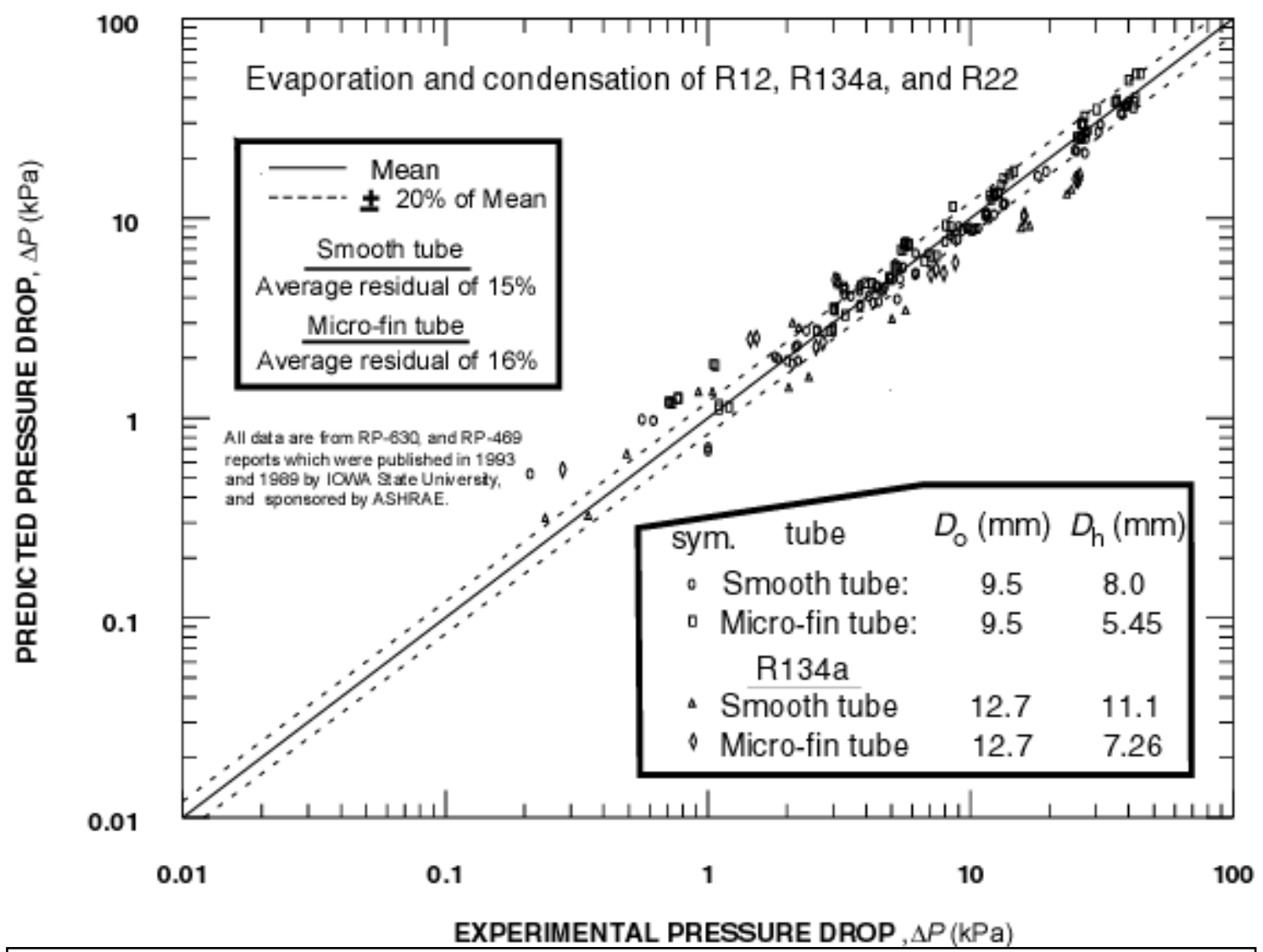

Figure 11 Predictions of Pate et al. (1991) pressure drop measurements with NIST correlation.

\section{Refrigerant/Lubricant Mixtures}

In practice, the refrigerant that circulates in refrigeration and air-conditioning equipment contains a small fraction of lubricant. For the present analysis, it was assumed that the lubricant alters the pressure drop solely through an increase in the viscosity of the liquid in the tube. As the vapor quality increases, the preferential evaporation of the refrigerant causes the local concentration of lubricant, and hence, the viscosity of the liquid to increase.

The pressure drop of an refrigerant/lubricant mixture was predicted using the pure refrigerant pressure drop correlation (Eq. (15)) with two modifications. These modifications accommodate the influence of the lubricant on the vapor quality and the liquid Reynolds number. 
The vapor quality for the refrigerant/lubricant mixture and the mass fraction of the liquid refrigerant flow rate in the total refrigerant flow rate is calculated by Eqs. (17) and (18), respectively.

$$
\begin{gathered}
x=\frac{G_{r e f, g}}{G_{t o t}}=\frac{G_{r e f, g}}{G_{r e f, g}+G_{r e f, f}+G_{o i l}} \\
(1-x)=\frac{G_{r e f, f}}{G_{t o t}}=\frac{G_{r e f, f}+G_{o i l}}{G_{r e f, g}+G_{r e f, f}+G_{o i l}}
\end{gathered}
$$

The liquid Reynolds number for the refrigerant/lubricant mixture, $\mathrm{Re}_{m}$, is calculated using the local viscosity of the liquid (refrigerant/lubricant mixture) on the tube wall:

$$
\operatorname{Re}_{m}=\frac{G D_{h}}{\mu_{m}}
$$

The Yokozeki (1994) viscosity model was selected for use here because of its success in predicting measurements of refrigerant/lubricant mixture viscosities. For example, Michels et al. (1996) recently verified the Yokozeki mixing model with measured viscosities for several refrigerant/lubricant mixtures: R22/AB 150, R134a/POE-68, and R410A/PVE-68. The Yokozeki (1994) viscosity mixture model is given as:

$$
\begin{array}{r}
\ln \mu_{m}=\sum_{i} \xi_{i} \ln \mu_{i}=\xi_{\text {ref }} \ln \mu_{r e f}+\xi_{\text {oil }} \ln \mu_{\text {oil }} \\
\text { where } \xi_{i}=\frac{W_{i}^{k} \Psi_{i}}{\sum_{j} W_{j}^{k} \Psi_{j}}
\end{array}
$$

$\Psi_{i}$ and $W_{i}$ are the mole fraction and molecular weight of component $\mathrm{i}$, respectively. The exponent $\mathrm{k}$ is an empirical parameter, often modeled as a function of a low order polynomial in the temperature. Each refrigerant/lubricant mixture has a particular value of k. However, Yokozeki (1994) found that setting $\mathrm{k}=0.58$ gave accurate predictions for many refrigerant/lubricant mixtures. Consequently, the value of $\mathrm{k}$ was taken as 0.58 in the present pressure drop model. 


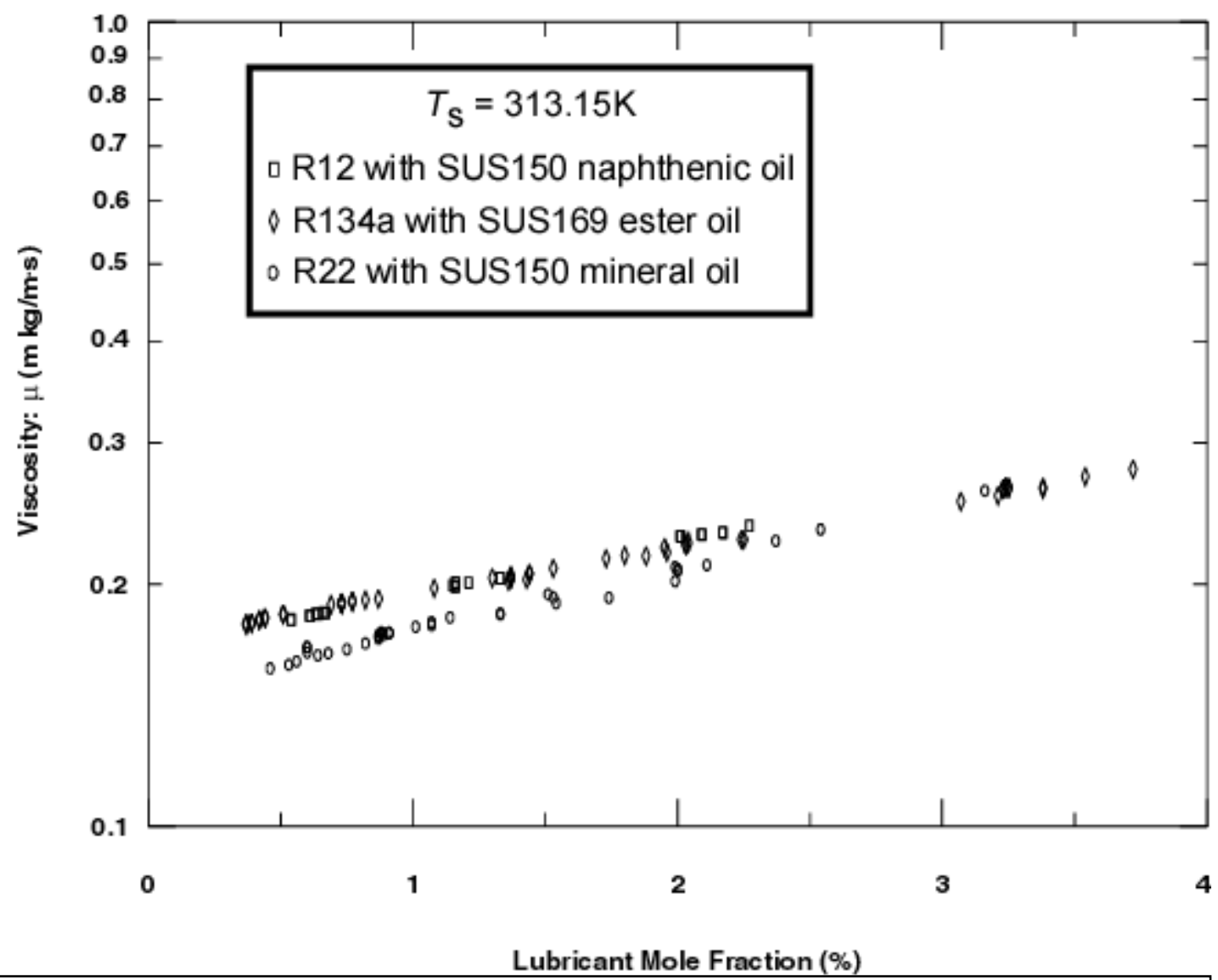

Figure 12 Lubricant viscosity as a function of mole fraction

Figure 12 shows viscosity of three refrigerant/lubricant mixtures that were calculated from the Yokozeki model. The viscosities of R12/naphthenic 150 SUS, R134a /169 SUS ester-m, and R22/mineral SUS150 are shown as a function of the lubricant mole fraction. The viscosity of the mixtures increase nearly linearly with respect to the lubricant mole fraction on a logarithmic viscosity scale.

If we consider only the liquid state of refrigerant and lubricant, the mass fraction of the lubricant in the refrigerant/lubricant mixture is:

$$
w_{\text {layer }, \text { ol }}=\frac{m_{\text {oil }}}{m_{\text {oil }}+m_{r e f, f}}
$$


The lubricant mass fraction is linearly averaged over the length of the tube. Equation 20 requires the molar fraction of the mixture as input. The mass fraction can be converted to molar fraction by the following equation:

$$
\Psi=\frac{w_{\text {layer }, \text { oil }}\left(\frac{m_{\text {ref }}}{m_{\text {oil }}}\right)}{1-w_{\text {layer }, \text { oil }}+w_{\text {layer }, \text { oil }}\left(\frac{m_{\text {ref }}}{m_{\text {oil }}}\right)}
$$

Figure 13 illustrates that, for fixed mass velocity, the Reynolds number is reduced by approximately $40 \%$ of the pure refrigerant value for a lubricant mole fraction of $3 \%$. This magnitude of Reynolds number reduction results in approximately a $7 \%$ increase in the friction factor. Consequently, the lubricant viscosity has a significant role in the prediction of the pressure drop.

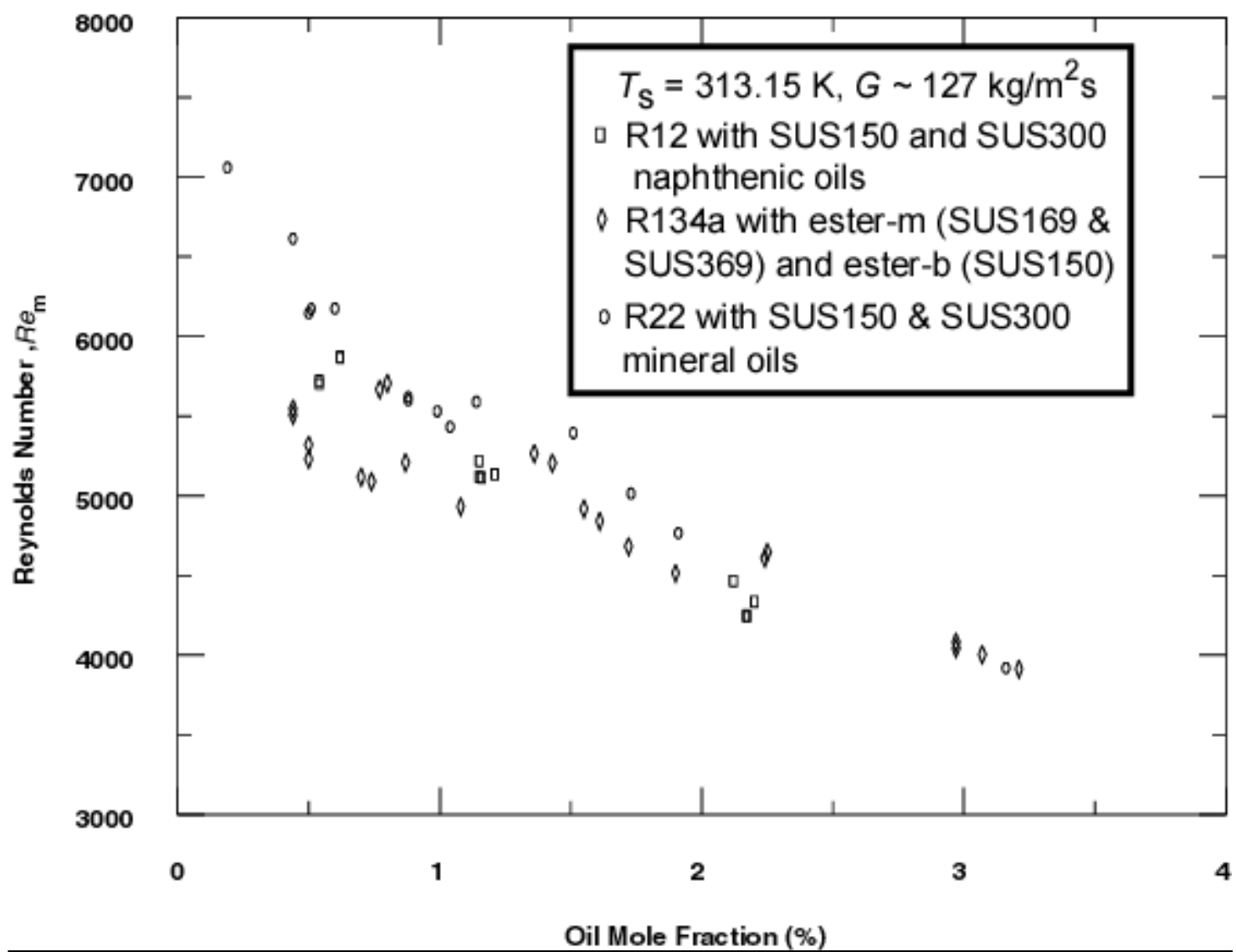

Figure 13 Reynolds Number for selected refrigerant/lubricant mixtures versus mole fraction 
The NIST pressure drop model for refrigerant/lubricant mixtures was compared to the data of Pate et al. (1991) which is given in Appendix E. Pate et al. (1991) measured convective condensation and evaporation pressure drops in smooth and micro-fin tubes for 28 different refrigerant/lubricant mixtures. The refrigerants were R12, R134a, and R22. Six mixtures of R12 and naphthenic oil were tested at two viscosities: SUS150, and SUS300. Fifteen mixtures of R134a and ester lubricant were tested at three lubricant viscosities: SUS169, SUS369, and SUS150. Finally, seven mixtures of R22 and mineral lubricant were tested at two lubricant viscosities: SUS150, and SUS300. Table 5 sumarizes the tubes that were tested and the various mass fraction of the mixtures.

Table 5. Refrigerant/lubricant mixture tests by Pate at al. (1991).

\begin{tabular}{|c|c|c|c|c|c|c|c|}
\hline \multirow{2}{*}{$\begin{array}{l}\text { Refrigerant } \\
\text { R12 }\end{array}$} & \multirow{3}{*}{$\begin{array}{l}\text { Tube } \\
\text { Smooth } 9.525 \mathrm{~mm} \\
\text { (3/8 in) }\end{array}$} & Lubricant & \multicolumn{5}{|c|}{ Lubricant Concentration (\%) } \\
\hline & & 150 SUS & $\overline{l 1.3}$ & $\overline{\overline{2.4}}$ & $4 \overline{4.9}$ & & \\
\hline & & $\begin{array}{l}\text { naphthenic } \\
300 \text { SUIS }\end{array}$ & 12 & 24 & 48 & & \\
\hline & & naphthenic & & & & & \\
\hline & Micro-fin $9.525 \mathrm{~mm}$ & $150 \mathrm{SUS}$ & 1.3 & 2.4 & 4.9 & & \\
\hline & & $\begin{array}{l}300 \text { SUS } \\
\text { naphthenic }\end{array}$ & 1.2 & 2.4 & 4.8 & & \\
\hline R134a & Smooth $9.525 \mathrm{~mm}$ & 169 SUS ester-m & 0.5 & 1.0 & 1.9 & & 5.0 \\
\hline & $(3 / 8$ in $)$ & 369 SUS ester-m & 0.6 & 1.1 & 2.4 & 5.0 & \\
\hline & & 150 SUS ester-b & 1.1 & 2.4 & 5.0 & & \\
\hline & Micro-fin $9.525 \mathrm{~mm}$ & US ester-m & 0.5 & 1.0 & 1.9 & 2.9 & 5.0 \\
\hline & $(3 / 8$ in $)$ & 369 SUS ester-m & $\begin{array}{l}0.6 \\
11\end{array}$ & $\begin{array}{l}1.1 \\
24\end{array}$ & 2.4 & 5.0 & \\
\hline & Smooth $12.7 \mathrm{~mm}$ & 169 SUS ester-m & 1.3 & 2.3 & 4.9 & & \\
\hline & $(1 / 21$ & & & & & & \\
\hline & $\begin{array}{l}\text { Micro-tin } 12.7 \mathrm{~mm} \\
(1 / 2 \text { in })\end{array}$ & 169 sus ester-m & 1.3 & 2.3 & 4.9 & & \\
\hline R22 & Smooth $9.525 \mathrm{~mm}$ & 150 SUS mineral & 1.2 & 2.5 & 4.9 & & \\
\hline & fin $0525 \mathrm{~mm}$ & 300 SUS mineral & 0.6 & 1.3 & 2.6 & 5.0 & \\
\hline & $(3 / 8$ in $)$ & 300 SUS mineral & 0.6 & 1.3 & 2.6 & 5.0 & \\
\hline
\end{tabular}




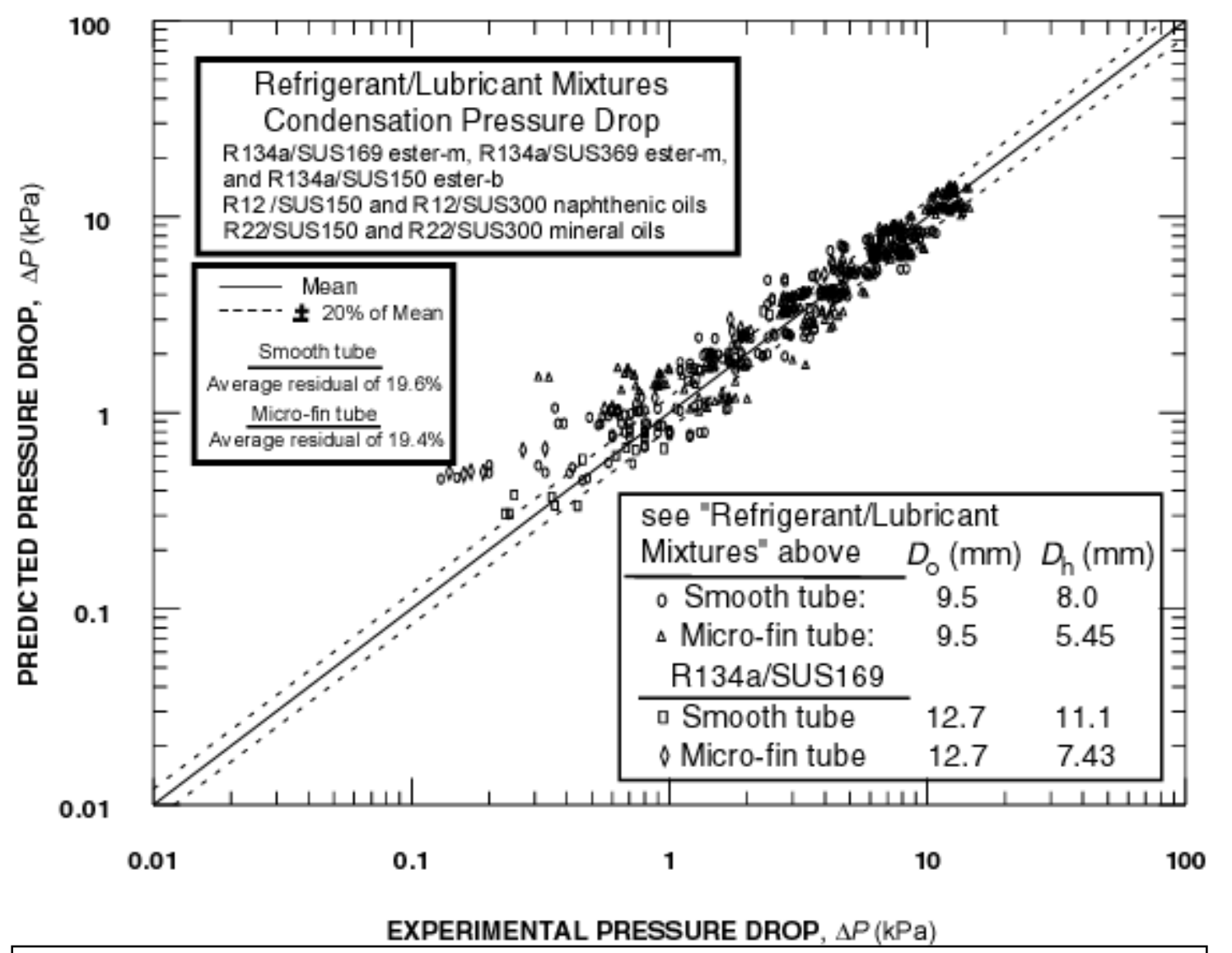

Figure 14 Predictions of Pate et al. (1991) condensation refrigerant/lubricant pressure drop measurements with NIST correlation

Figure 14 compares the condensation pressure drop of the refrigerant/lubricant mixtures measured by Pate et al. (1991) to the predicted pressure drop by the NIST correlation using a mixing viscosity rule. The figure shows that the present correlation predicts the measured data of the smooth and the micro-fin tubes to within $19.6 \%$, and $19.4 \%$, respectively. With the exception of the lowest pressure drops, the data scatter is centered about the mean of the predictions for both the smooth and micro-fin tubes and for all refrigerants.

Figure 15 compares the evaporation pressure drop of the refrigerant/lubricant mixtures measured by Pate et al. (1991) to the predicted pressure drop by the NIST correlation using a mixing viscosity rule. The figure shows that the present correlation predicts the measured data of the smooth and the micro-fin tubes to within $28.0 \%$ and $14.9 \%$, respectively. The data scatter was 
not centered about the mean for the evaporation data. The present pressure drop model tended to underpredict much of the data for all pressure drop ranges and refrigerants.

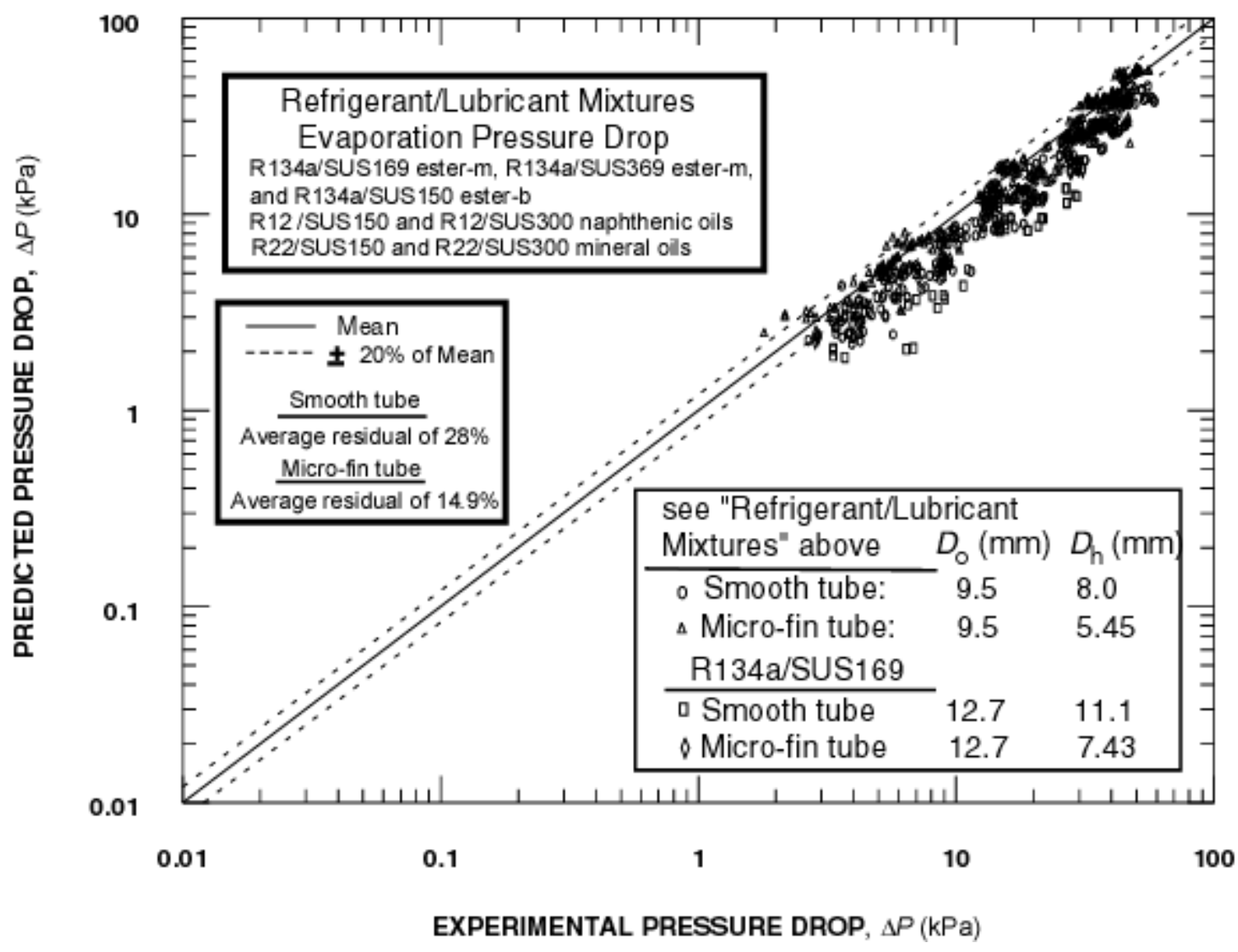

Figure 15 Predictions of Pate et al. (1991) evaporation refrigerant/lubricant pressure drop measurements with NIST correlation.

\section{CONCLUSIONS}

A pressure drop correlation for smooth and micro-fin tubes was developed based on the evaporation pressure drop correlation of Pierre (1964). The new correlation (Eq. (15)) is valid for both condensation and evaporation of pure refrigerants and refrigerant/lubricant mixtures. Two main modifications were made to the Pierre (1964) correlation to make it more general. First, the smooth tube diameter was replaced with the hydraulic diameter of the micro-fin tube. Second, the viscosity of the liquid in the Reynolds number was replaced by the refrigerant/lubricant viscosity. 
The new correlation was developed from NIST lubricant-free refrigerant pressure drop data in a micro-fin tube. The resulting correlation reproduced the NIST measurements to within $10.8 \%$. The pressure drop correlation was also validated with other pressure drop data that were not included in the correlation development. The lubricant-free refrigerant pressure drop data of Pate et al. (1991) and of Eckels et al. (1991 and 1993) for several different micro-fin tube geometries was predicted to within $18 \%$. The new correlation predicted the evaporative pressure drop data of Pate et al. (1991) and of Eckels et al. (1991) for refrigerant/lubricant mixtures in smooth and micro-fin tubes to within $28 \%$ and $15 \%$, respectively. The condensation pressure drop data of Pate et al. (1991) and of Eckels et al. (1991) for refrigerant/lubricant mixtures were predicted to within $19 \%$ for both smooth and micro-fin tubes.

\section{ACKNOWLEDGMENTS}

Mr. Jun-Young Choi's NIST sabbatical was funded by NIST and KTL, Korea. The authors thank Mr. Michael Kaul and Mr. Joaquim Goncalves for taking the evaporation and condensation pressure drop measurements at NIST, respectively. We also wish to thank Dr. Steve J. Eckels of the Kansas State University and Dr. Michael Pate of the Iowa State University for their experimental pressure drop data and helpful discussions. Also, Mr. Petur Thors and Mr. James Bogart of the Wolverine Tube Inc. supplied the Turbo-A micro-fin tube for tests at NIST and also provided geometric descriptions of all tubes involved in this study. Thanks goes to the following NIST personnel for their constructive criticism of the first draft of this manuscript: Dr. Vance Payne, Dr. James Olsen, and Mrs. Janet Land. 


\section{REFERENCES}

Bergles, A. E., and Luu, M. , 1980a, “Augmentation of in-tube condensation of R-113”, Heat Transfer Laboratory Report No. HTL-23, ISU-ERI-Ames-80175, Iowa State University.

Bergles, A. E., and Luu, M. , 1980b, "Enhancement of Horizontal In-tube Condensation of Refrigerant 113”, ASHRAE Transactions, Vol. 86, Part 1, pp. 293-312.

Bergles, A. E., and Luu, M. , 1979, "Enhancement of In-tube Condensation of R-113", ASHRAE Transactions, Vol. 85, Part 2, pp. 293-312.

Blasius, H. Z., 1913, “Das Ahnlichkeitsgesetz bei Reibungsvorgangen in Flussigkeiten” Forschg. Arb. Ing. Wes., No. 131, Berlin.

Blasius, H. Z., 1908, "Math. Physs., 56, 1", English Translation in National Advisory Committee for Aeronautics Technical Memo No. 1256.

Butterworth, D. , 1975, “A Comparison of Some Void-Fraction Relationships for Co-current Gas-Liquid Flow", International Journal of Multiphase Flow, pp. 845-850.

Chishom, D. , 1968, “The Influence of Mass Velocity on Friction Pressure Gradients During Steam-Water Flow", Presented at 1968 Thermodynamics and Fluid Mechanics Convention of the Institute of Mechanical Engineers held in Bristol, UK, March.

Collier, John G., and Thome, John R. , 1994, "Convective Boiling and Condensation 3rd Edition”, Clarendon Press, Oxford, pp. 34-68.

Dukler, A. E., M. Wicks, and R. G. Cleveland, 1949, "Frictional Pressure Drop in Two-Phase Flow: B. An Approach Through Similarity Analysis.” AIChE Journal 10.

Eckel, S. J., and Pate, M. B. , 1991, "In-Tube Evaporation and Condensation of RefrigerantLubricant Mixtures of HFC-134a and CFC-12” ASHRAE Transaction 97-2.

Eckels, S. J., Doerr, T. M., Pate, M. B. , 1993, "Heat Transfer and Pressure Drop During Condensation and Evaporation of HFC-134a/Oil Mixtures in Smooth and Micro-tube(RP-630)" ASHRAE RP-630, Engineering Research Institute, Iowa State University,.

Ely, J. F., and Hanley, H. J. M. , 1981b, "Prediction of Transport Properties. 1. Viscosity of Fluids and Mixtures", Ind. Eng. Chem. Fund., Vol. 22, pp. 90-97.

Ely, J. F., and Hanley, H. J. M. , 1983, "Prediction of Transport Properties. 2. Thermal Conductivity of Pure Fluids and Mixtures”, Ind. Eng. Chem. Fund., Vol. 22, pp. 90-97.

Fukushima, T., and M. Kudou , “ Heat Transfer Coefficient and Pressure Drops for Forced 
Gallagher, J., M. Huber, G. Morrison and M. McLinden,1993, NIST Standard Reference Database 23, NIST thermodynamic properties of refrigerants and refrigerant mixtures database (REFPROP), version 4.01. Gaithersburg, MD, Standard Reference Data Program, National Institute of Standards and Technology.

Hamilton L. C., 1992, Regression with Graphics: A Second Course in Applied Statistics, Duxbury Press, Belmont.

Jung, D. S., and Radermacher, R. , 1989, "Prediction of Pressure Drop During Horizontal Annual Flow Boiling of Pure and Mixed Refrigerants”, Int. J. Heat Mass Transfer, Vol. 32, No. 12, pp. 2435-2446.

Kaul, M. P., Kedzierski, M. A., and Didion, D. A., 1996, "Horizontal Flow Boiling of Alternative Refrigerants Within a Fluid Heated Micro-Fin Tube”, Process, Enhanced, and Mutiphase Heat Transfer: A Festschrift for A. E. Bergles, Begell House, Inc., New York, pp. 167-173.

Kedzierski, M. A., and Goncalves, J. M., 1999, "Horizontal Convective Condensation of Alternative Refrigerants Within a Micro-Fin Tube," Journal of Enhanced Heat Transfer, Vol. 6, pp. 161-178.

Kedzierski, M., A, and Goncalves, J. M., 1997, "Horizontal Convective Condensation of Alternative Refrigerants Within a Micro-Fin Tube”, NISTIR 6095, pp. 12-16.

Khanpara, J. C., Bergles, A. E., and Pate, M. B., 1987, "A Comparison of In-Tube Evaporation of R-113 in Electrically Heated and Fluid Heated Smooth and Inner-Fin Tubes" Advanced in Enhanced Heat Transfer-HTD-68, pp. 34-45.

Koops, P. E., 1992, "Comparison of Heat Transfer and Pressure Drop Characteristics of Refrigerants 134a and 12 During Condensation Inside Smooth and Internally Finned Tubes", Master thesis, Kansas State University.

Lockhart, R. W., and Martnelli, R. C., 1949, "Proposed Correlation of Data for Isothermal Two-Phase, Two-Component Flow in Pipes." Chemical Engineering Progress 45 (1), pp. 39-48.

Martinelli, R.C. and Nelson, D.B., (1948), "Prediction of Pressure Drop During Forced Circulation Boiling of Water", ASME Transactions, No. 6.

Michels, H., Harvey, and Sienel, Tobias, H. , 1996, "Viscosity Modeling of Refrigerant/Lubricant Mixtures”, The Proc. Int. Ref. Conf., Purdue, pp. 96-105. 
Mori, Y., Nakayama, W. , 1983, "High-Performance Mist Cooled Condensers for Geothermal Binary Cycle Plants", Heat Transfer in Energy Problem, pp. 211-218.

Pierre, B. , 1964, "Flow Resistance With Boiling Refrigerants-Part 1", ASHRAE Journal,Vol. 6, No. 9, pp. 58-65.

Signal, L. C., Sharma, C. P., and Varma, H. K. , 1983, "Pressure Drop During Forced Convection Boiling of Binary Refrigerant Mixtures”, Int. J. Multiphase Flow, Vol. 9, No. 3, pp. 309-323.

Schlager, L.M., Pate, M.B., Bergles, A.E., 1989, "Effect of Oil on Heat Transfer and Pressure Drop Inside Augmented Tubes During Condensation and Evaporation of Refrigerants (RP-469)" ASHRAE RP-469, Engineering Research Institute, Iowa State University.

Tichy, J.A., Duque-Rivera, J., Macken, N. A., and Duval, W. M. B. , 1986, “An Experimental Investigation of Pressure Drop in Ford-Convection Condensation and Evaporation of LubricantRefrigerant Mixture" ASHRAE Transactions 92, pp. 461-472.

Torikoshi, K. and Kawabata, K. , 1992, "Heat Transfer and Pressure Drops Characteristics of HFC-134a in a Horizontal Heat Transfer Tube" In Proceedings: 1992 International Refrigeration Conference-Energy Efficiency and New Refrigerants 1, pp. 167-176.

Wang, C., Kuo, C. S., Chang, Y., and Lu, D. C., 1996, “Two-Phase Flow Heat Transfer and Friction Characteristics of R22 and R-407C", ASHRAE Transactions, Vol. 102, Pt. 1., pp. 830838.

Webb, R. L. , 1994, Principles of Enhanced Heat Transfer, Wiley Interscience.

Yokozeki, A., M., 1994, "Solubility and Viscosity of Refrigerant-Lubricant Mixtures", Proc. Int. Ref. Conf., Purdue University. 


\section{APPENDIX A}

\section{Condensation pressure drop of NIST in micro-fin tube(file: nist_con.in)}

\begin{tabular}{|c|c|c|c|c|c|c|c|c|c|c|c|}
\hline \multicolumn{2}{|c|}{ 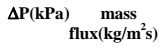 } & $\mathrm{z}(\mathrm{m})$ & $\mathrm{x}_{\mathrm{in}}$ & $\mathbf{x}_{\text {out }}$ & $P_{\text {in }}(\mathbf{k P a})$ & $\mathbf{P}_{\mathrm{c}}(\mathbf{k P a})$ & $h_{f_{8}(k J / k g)}$ & $v_{f}\left(m^{3} / \mathbf{k g}\right)$ & $v_{g}\left(m^{3} / k g\right)$ & $\begin{array}{l}\mu_{\mathrm{f}} \\
\left(\mathbf{x} 10^{-7}, \mathrm{~N}\right.\end{array}$ & $\underset{\left.\mathbf{a s}^{2}\right)}{\mu_{\mathrm{g}}}$ \\
\hline \multicolumn{12}{|c|}{ R32 } \\
\hline 1.184 & 191.38 & 1.587 & 0.487 & 0.223 & 1729.6 & 5795 & 270.78 & 0.00104 & 0.02069 & 1160 & 130 \\
\hline 0.454 & 191.38 & 1.588 & 0.203 & 0.106 & 1728.26 & 5795 & 270.83 & 0.00104 & 0.020705 & 1160 & 129 \\
\hline 0.65 & 137.45 & 1.587 & 0.494 & 0.233 & 2042.22 & 5795 & 256.95 & 0.00108 & 0.01718 & 1067 & 134 \\
\hline 0.753 & 157.35 & 1.587 & 0.506 & 0.244 & 2217.02 & 5795 & 249.44 & 0.00109 & 0.01563 & 1022 & 137 \\
\hline $\begin{array}{l}1.927 \\
\end{array}$ & 268.26 & 1.587 & 0.488 & 0.211 & 1995.53 & 5795 & 259.02 & 0.00107 & 0.01764 & 1080 & 133 \\
\hline 0.729 & 268.26 & 1.588 & 0.191 & 0.086 & 1992.87 & 5795 & 259.11 & 0.00107 & 0.017665 & 1081 & 133 \\
\hline 2.165 & 306.95 & 1.587 & 0.505 & 0.231 & 2285.62 & 5795 & 246.55 & 0.0011 & 0.01509 & 1006 & 137 \\
\hline 1.324 & 306.95 & 1.588 & 0.211 & 0.108 & 2282.82 & 5795 & 246.66 & 0.0011 & 0.015115 & 1006 & 137 \\
\hline 1.638 & 232.55 & 1.587 & 0.481 & 0.212 & 1835.54 & 5795 & 266.05 & 0.00105 & 0.019375 & 1126 & 131 \\
\hline 0.666 & 232.55 & 1.588 & 0.192 & 0.09 & 1833.43 & 5795 & 266.12 & 0.00105 & 0.019395 & 1127 & 131 \\
\hline 1.513 & 145.72 & 1.587 & 0.974 & 0.823 & 2117.78 & 5795 & 253.72 & 0.00108 & 0.016485 & 1047 & 135 \\
\hline 0.805 & 145.72 & 1.588 & 0.798 & 0.509 & 2115.3 & 5795 & 253.81 & 0.00108 & 0.016505 & 1047 & 135 \\
\hline 1.168 & 145.77 & $\begin{array}{l}1.587 \\
\end{array}$ & 0.966 & 0.82 & 2112.15 & 5795 & 253.95 & 0.00108 & 0.016535 & 1048 & 135 \\
\hline 1.166 & 145.77 & 1.588 & 0.795 & 0.513 & 2109.99 & 5795 & 254.05 & 0.00108 & 0.016555 & 1049 & 135 \\
\hline 1.659 & 173.34 & 1.587 & 0.984 & 0.828 & 2292.67 & 5795 & 246.25 & 0.0011 & 0.015035 & 1004 & 138 \\
\hline 1.063 & 173.34 & 1.588 & 0.802 & 0.505 & 2289.74 & 5795 & 246.36 & 0.0011 & 0.015055 & 1005 & 138 \\
\hline 2.132 & 183.11 & $\begin{array}{l}1.580 \\
1.587\end{array}$ & 0.975 & 0.815 & 1677.22 & 5795 & $\begin{array}{l}270.50 \\
273.19\end{array}$ & 0.00104 & 0.02141 & 1178 & 129 \\
\hline 1.563 & 165.22 & 1.587 & 0.971 & 0.821 & 1951.44 & 5795 & 260.94 & 0.00107 & 0.018095 & 1092 & 133 \\
\hline 1.331 & 165.22 & 1.588 & 0.795 & 0.508 & 1948.81 & 5795 & 261.05 & 0.00107 & 0.01812 & 1093 & 133 \\
\hline 0.595 & 88.37 & 1.587 & 0.962 & 0.808 & 2015.93 & 5795 & 258.1 & 0.00107 & 0.017435 & 1074 & 134 \\
\hline 0.576 & 91.24 & 1.587 & 0.963 & 0.809 & 2176.61 & 5795 & 251.17 & 0.00109 & 0.015965 & 1032 & 136 \\
\hline 0.556 & 91.99 & 1.587 & 0.961 & 0.805 & 2304.45 & 5795 & 245.72 & 0.0011 & 0.01494 & 1001 & 138 \\
\hline 1.183 & 125.1 & 1.588 & 0.778 & 0.483 & 1564.89 & 5795 & 278.34 & 0.00103 & 0.023075 & 1217 & 127 \\
\hline 5.207 & 335.11 & 1.587 & 0.9999 & 0.834 & 2136.18 & 5795 & 253.72 & 0.00108 & 0.01642 & 1037 & 135 \\
\hline 4.373 & 335.11 & 1.588 & 0.807 & $\begin{array}{l}0.031 \\
0.51\end{array}$ & 2125.25 & 5795 & 253.49 & 0.00108 & 0.016435 & 1046 & 135 \\
\hline 3.181 & 251.9 & 1.587 & 0.988 & 0.793 & 2180.81 & 5795 & 251.06 & 0.00109 & 0.015945 & 1031 & 136 \\
\hline 2.854 & 242.1 & 1.587 & 0.987 & 0.826 & 2170.15 & 5795 & 251.51 & 0.00109 & 0.016035 & 1034 & 136 \\
\hline 2.996 & 251.67 & 1.587 & 0.989 & 0.821 & 2275.03 & 5795 & 247.04 & 0.0011 & 0.01518 & 1008 & 137 \\
\hline 2.498 & 251.67 & 1.588 & 0.794 & 0.497 & 2269.07 & 5795 & 247.28 & 0.0011 & 0.015225 & 1009 & 137 \\
\hline 3.831 & 255.34 & 1.587 & 0.988 & 0.803 & 1852.6 & 5795 & 265.37 & 0.00106 & 0.0192 & 1122 & 131 \\
\hline 3.269 & 255.34 & 1.588 & 0.774 & 0.462 & 1844.81 & 5795 & 265.7 & 0.00106 & 0.019285 & 1124 & 131 \\
\hline 3.99 & 281.15 & 1.587 & 0.988 & 0.816 & 2111.22 & 5795 & 254.08 & 0.00108 & 0.016555 & 1049 & 135 \\
\hline 3.39 & 281.15 & 1.588 & 0.789 & 0.489 & 2103.06 & 5795 & 254.42 & 0.00108 & 0.01663 & 1052 & 135 \\
\hline 4.351 & 268.85 & 1.587 & 0.948 & 0.786 & 1837.02 & 5795 & 266.07 & 0.001055 & 0.019385 & 1126 & 131 \\
\hline 3.779 & 268.85 & 1.588 & 0.76 & 0.476 & 1828.14 & 5795 & 266.46 & 0.00105 & 0.019485 & 1129 & 131 \\
\hline 1.016 & 120.12 & $\begin{array}{l}1.587 \\
\end{array}$ & 0.961 & 0.783 & 1850.27 & 5795 & 265.38 & 0.00106 & 0.0192 & 1122 & 131 \\
\hline 0.638 & 120.12 & 1.588 & 0.754 & 0.455 & 1848.71 & 5795 & 265.44 & 0.00106 & 0.01922 & 1122 & 131 \\
\hline $\begin{array}{l}1.290 \\
1.295\end{array}$ & 138.46 & $\begin{array}{l}1.580 \\
1.587\end{array}$ & 0.958 & 0.752 & 1782.28 & 5795 & 268.42 & 0.00105 & 0.02002 & 1142 & 130 \\
\hline 0.872 & 138.46 & 1.588 & 0.719 & 0.393 & 1780.02 & 5795 & 268.52 & 0.00105 & 0.02004 & 1143 & 130 \\
\hline 0.864 & $\begin{array}{l}109.90 \\
109\end{array}$ & 1.587 & 0.965 & 0.797 & 1805.15 & 5795 & 267.39 & 0.00105 & 0.019735 & 1135 & 131 \\
\hline 0.542 & 109.9 & 1.588 & 0.77 & 0.475 & 1803.87 & 5795 & 267.44 & 0.00105 & 0.01975 & 1136 & 131 \\
\hline 1.371 & 206.38 & $\begin{array}{l}1.587 \\
\end{array}$ & 0.486 & 0.213 & 1768.19 & 5795 & 269.05 & 0.00105 & 0.020195 & 1147 & 130 \\
\hline 0.441 & 206.38 & 1.588 & 0.193 & 0.089 & 1766.41 & 5795 & 269.11 & 0.00105 & 0.020215 & 1148 & 130 \\
\hline $\begin{array}{l}0.1446 \\
1.146\end{array}$ & 196.03 & $\begin{array}{l}1.580 \\
1.587\end{array}$ & 0.502 & 0.238 & 2068.98 & 5795 & 255.81 & 0.00108 & 0.016925 & $\begin{array}{l}1060 \\
1060\end{array}$ & 134 \\
\hline 0.387 & 196.03 & 1.588 & 0.218 & 0.114 & 2067.64 & 5795 & 255.85 & 0.00108 & 0.01694 & 1060 & 134 \\
\hline $\begin{array}{l}1.391 \\
1.392\end{array}$ & 212.03 & $\begin{array}{l}1.580 \\
1.587\end{array}$ & 0.489 & 0.218 & 1831.64 & 5795 & 266.22 & 0.00105 & 0.01942 & 1127 & 131 \\
\hline 0.488 & 212.03 & 1.588 & 0.198 & 0.096 & 1829.91 & 5795 & 266.28 & 0.00105 & 0.019435 & 1128 & 131 \\
\hline $\begin{array}{l}1.400 \\
1.27\end{array}$ & 198.06 & $\begin{array}{l}1.587 \\
1.587\end{array}$ & 0.54 & 0.312 & 2221.95 & 5795 & 249.24 & 0.00109 & 0.015595 & 1021 & 137 \\
\hline 0.53 & 198.06 & 1.588 & 0.295 & 0.206 & 2220.31 & 5795 & 249.3 & 0.00109 & 0.015605 & 1021 & 137 \\
\hline 0.602 & 136.1 & 1.587 & 0.431 & 0.15 & 1620.17 & 5795 & 275.76 & 0.00103 & 0.02222 & 1197 & 128 \\
\hline 0.537 & 122.41 & 1.587 & 0.475 & 0.213 & 1832.68 & 5795 & 266.15 & 0.00105 & 0.019405 & 1127 & 131 \\
\hline
\end{tabular}

\section{R125}

\begin{tabular}{|c|c|c|c|c|c|c|c|c|c|c|c|}
\hline 1.639 & 226.75 & 1.587 & 0.9999 & 0.873 & 1553.3 & 3629 & 106.94 & 0.00086 & 0.00979 & 1326 & 151 \\
\hline 1.296 & 226.75 & 1.588 & 0.847 & 0.549 & 1550.25 & 3629 & 105.4 & 0.00086 & 0.009665 & 1358 & 150 \\
\hline 1.022 & 226.76 & 1.588 & 0.868 & 0.581 & 1795.63 & 3629 & 98.39 & 0.00089 & 0.00811 & 1254 & 157 \\
\hline 2.454 & 298.89 & 1.587 & 0.943 & 0.741 & 1728.21 & 3629 & 100.34 & 0.00088 & 0.0085 & 1282 & 155 \\
\hline 1.562 & 298.89 & 1.588 & 0.711 & 0.421 & 1723.77 & 3629 & 100.45 & 0.00088 & 0.00852 & 1284 & 155 \\
\hline 4.074 & 468.06 & 1.587 & 0.962 & 0.758 & 1979.86 & 3629 & 93.17 & 0.00091 & 0.007185 & 1189 & 164 \\
\hline 2.811 & 468.06 & 1.588 & 0.727 & 0.412 & 1971.73 & 3629 & 93.39 & 0.00091 & 0.007215 & 1191 & 163 \\
\hline 3.411 & 458.15 & 1.587 & 0.958 & 0.757 & 2192.28 & 3629 & 86.97 & 0.00094 & 0.00628 & 1120 & 171 \\
\hline 2.271 & 458.15 & 1.588 & 0.727 & 0.418 & 2185.57 & 3629 & 87.15 & 0.00094 & 0.0063 & 1122 & 171 \\
\hline 5.599 & 435.47 & 1.587 & 0.942 & 0.713 & 1439.96 & 3629 & 108.65 & 0.00085 & 0.01055 & 1413 & 146 \\
\hline 3.659 & 435.47 & 1.588 & 0.68 & 0.348 & 1428.75 & 3629 & 108.95 & 0.00085 & 0.010635 & 1418 & 146 \\
\hline 4.88 & 444.25 & 1.587 & 0.958 & 0.744 & 1641.42 & 3629 & 102.86 & 0.00087 & 0.00905 & 1318 & 152 \\
\hline 3.256 & 444.25 & 1.588 & 0.713 & 0.395 & 1631.62 & 3629 & 103.12 & 0.00087 & 0.009115 & 1322 & 152 \\
\hline 4.506 & 448.23 & 1.587 & 0.962 & 0.763 & 1767.23 & 3629 & 99.27 & 0.00089 & 0.00828 & 1267 & 156 \\
\hline 3.09 & 448.23 & 1.588 & 0.734 & 0.43 & 1758.22 & 3629 & 99.51 & 0.00089 & 0.008325 & 1270 & 156 \\
\hline 1.079 & 193.96 & 1.587 & 0.955 & 0.759 & 1908.77 & 3629 & 95.16 & 0.00091 & 0.007515 & 1212 & 161 \\
\hline 0.535 & 193.96 & 1.588 & 0.729 & 0.427 & 1907.28 & 3629 & 95.19 & 0.00091 & 0.00752 & 1213 & 161 \\
\hline 1.868 & 223.59 & 1.587 & 0.985 & 0.82 & 1392.92 & 3629 & 109.94 & 0.00084 & 0.01094 & 1436 & 145 \\
\hline 1.397 & 223.59 & 1.588 & 0.793 & 0.472 & 1389.45 & 3629 & 110.04 & 0.00084 & 0.01096 & 1438 & 145 \\
\hline 3.285 & 350.59 & 1.588 & 0.806 & 0.51 & 1450.03 & 3629 & 108.32 & 0.00085 & 0.010455 & 1407 & 147 \\
\hline 3.696 & 358.1 & 1.587 & 0.994 & 0.806 & 1589.53 & 3629 & 104.32 & 0.00087 & 0.0094 & 1341 & 151 \\
\hline 2.734 & 358.1 & 1.588 & 0.777 & 0.45 & 1582.23 & 3629 & 104.52 & 0.000865 & 0.009445 & 1344 & 151 \\
\hline 2.281 & 296.49 & 1.587 & 0.962 & 0.821 & 1866.72 & 3629 & 96.38 & 0.0009 & 0.00773 & 1229 & 160 \\
\hline 1.6 & 296.49 & 1.588 & 0.797 & 0.532 & 1862.61 & 3629 & 96.49 & 0.0009 & 0.00775 & 1230 & 159 \\
\hline 2.34 & 331.97 & 1.587 & 0.9999 & 0.826 & 2047.1 & 3629 & 91.77 & 0.00092 & 0.006915 & 1157 & 166 \\
\hline 1.492 & 331.97 & 1.588 & 0.797 & 0.473 & 2042.72 & 3629 & 91.31 & 0.00092 & 0.00689 & 1167 & 166 \\
\hline 1.398 & 332.22 & 1.588 & 0.813 & 0.519 & 2211.48 & 3629 & 86.36 & 0.00095 & 0.0062 & 1114 & 172 \\
\hline 1.048 & 278.39 & 1.587 & 0.556 & 0.289 & 2255.73 & 3629 & 85.03 & 0.00095 & 0.00603 & 1101 & 174 \\
\hline 0.485 & 278.39 & 1.588 & 0.267 & 0.14 & 2254.51 & 3629 & 85.06 & 0.00095 & 0.00603 & 1101 & 174 \\
\hline 0.799 & 334.65 & 1.588 & 0.224 & 0.111 & 1546.81 & 3629 & 105.49 & 0.00086 & 0.00969 & 1360 & 150 \\
\hline 2.012 & 346.89 & 1.587 & 0.514 & 0.236 & 1408.99 & 3629 & 109.47 & 0.00084 & 0.01079 & 1427 & 145 \\
\hline 1.682 & 341.31 & 1.587 & 0.532 & 0.275 & 1821 & 3629 & 97.68 & 0.00089 & 0.007965 & 1245 & 158 \\
\hline 2.64 & 455.98 & 1.587 & 0.537 & 0.281 & 1818.86 & 3629 & 97.76 & 0.00089 & 0.007985 & 1246 & 158 \\
\hline 3.268 & 458.62 & 1.587 & 0.527 & 0.261 & 1451.79 & 3629 & 108.26 & 0.00085 & 0.01044 & 1406 & 147 \\
\hline 0.73 & 213.82 & 1.587 & 0.478 & 0.207 & 1394.82 & 3629 & 109.86 & 0.00084 & 0.010915 & 1435 & 145 \\
\hline 1.446 & 394.7 & 1.587 & 0.509 & 0.232 & 2137.74 & 3629 & 88.53 & 0.00094 & 0.006485 & 1136 & 169 \\
\hline \multicolumn{12}{|c|}{ R410A } \\
\hline 20 & 32 & 1.587 & 0.53 & 0.269 & 2081.02 & 4950 & 182.03 & 0.00098 & 0.01224 & 1091 & 143 \\
\hline
\end{tabular}




\begin{tabular}{|c|c|c|c|c|c|c|c|c|c|c|c|}
\hline 0.842 & 324.03 & 1.588 & 0.249 & 0.142 & 2078.15 & 4950 & 183.11 & 0.00098 & 0.01231 & 1091 & 143 \\
\hline 6 & 443.02 & 1.587 & 0.646 & 0.466 & 1714.52 & 4950 & 194.14 & 0.00094 & 0.015215 & 1213 & 137 \\
\hline 5.493 & 454.96 & 1.587 & 0.647 & 0.444 & 1845.7 & 4950 & 189.55 & 0.00095 & 0.01401 & 1167 & 139 \\
\hline 3.987 & 370.41 & 1.587 & 0.614 & 0.401 & 1725.32 & 4950 & 193.96 & 0.00094 & 0.015115 & 1209 & 137 \\
\hline 2.526 & 370.41 & 1.588 & 0.385 & 0.298 & 1718.31 & 4950 & 195.07 & 0.00094 & 0.015235 & 1209 & 137 \\
\hline 0.589 & 137.62 & 1.587 & 0.508 & 0.258 & 1695.22 & 4950 & 195.64 & 0.00094 & 0.01544 & 1218 & 136 \\
\hline 0.191 & 137.63 & 1.588 & 0.265 & 0.176 & 1847.28 & 4950 & 191.06 & 0.00096 & 0.01408 & 1163 & 139 \\
\hline 0.82 & 173.07 & 1.587 & 0.556 & 0.322 & 2018.57 & 4950 & 183.96 & 0.00097 & 0.012655 & 1110 & 142 \\
\hline 0.325 & 173.07 & 1.588 & 0.306 & 0.225 & 2017.8 & 4950 & 184.89 & 0.00097 & 0.012715 & 1109 & 142 \\
\hline 3.262 & 264.23 & 1.587 & 0.9999 & 0.822 & 1669.72 & 4950 & 198.21 & 0.00093 & 0.015755 & 1220 & 136 \\
\hline 2.847 & 264.23 & 1.588 & 0.793 & 0.492 & 1663.1 & 4950 & 195.43 & 0.00094 & 0.01568 & 1232 & 136 \\
\hline 2.764 & 270.98 & 1.587 & 0.959 & 0.806 & 2078.14 & 4950 & 179.69 & 0.000975 & 0.01211 & 1094 & 144 \\
\hline 2.156 & 270.98 & 1.588 & 0.782 & 0.523 & 2072.79 & 4950 & 181.04 & 0.00098 & 0.01222 & 1095 & 143 \\
\hline 7.245 & 434.13 & 1.587 & 0.965 & 0.824 & 1850.3 & 4950 & 187.57 & 0.00095 & 0.013855 & 1167 & 139 \\
\hline 6.19 & 434.13 & 1.588 & 0.801 & 0.559 & 1835.01 & 4950 & 189.25 & 0.00095 & 0.01406 & 1171 & 139 \\
\hline 4.617 & 337.33 & 1.587 & 0.9999 & 0.831 & 1821.44 & 4950 & 195.55 & 0.00094 & 0.01455 & 1134 & 140 \\
\hline 3.833 & 337.33 & 1.588 & 0.8 & 0.485 & 1811.9 & 4950 & 190.19 & 0.00095 & 0.01426 & 1178 & 138 \\
\hline 6.938 & 457.24 & 1.587 & 0.995 & 0.849 & 2032.23 & 4950 & 181.16 & 0.00097 & 0.01244 & 1109 & 143 \\
\hline 5.969 & 457.24 & 1.588 & 0.825 & 0.574 & 2017.35 & 4950 & 182.81 & 0.00097 & 0.01261 & 1112 & 142 \\
\hline 5.165 & 468.01 & 1.588 & 0.817 & 0.526 & 2180.13 & 4950 & 177.33 & 0.00099 & 0.011525 & 1065 & 145 \\
\hline 6.045 & 376.33 & 1.587 & 0.968 & 0.819 & 1738.62 & 4950 & 191.45 & 0.00094 & 0.01485 & 1206 & 137 \\
\hline 5.212 & 376.33 & 1.588 & 0.795 & 0.545 & 1725.97 & 4950 & 193.1 & 0.00094 & 0.015055 & 1209 & 137 \\
\hline 1.222 & 164.7 & 1.587 & 0.9999 & 0.828 & 1817.49 & 4950 & 192.77 & 0.00094 & 0.014305 & 1169 & 139 \\
\hline 0.952 & 164.7 & 1.588 & 0.799 & 0.488 & 1815.41 & 4950 & 189.99 & 0.00095 & 0.01421 & 1177 & 138 \\
\hline 1.039 & 166.75 & 1.587 & 0.9999 & 0.86 & 2202.67 & 4950 & 181.67 & 0.00098 & 0.01163 & 1028 & 146 \\
\hline 0.646 & 166.75 & 1.588 & 0.833 & 0.528 & 2201.02 & 4950 & 176.46 & 0.00099 & 0.011365 & 1058 & 146 \\
\hline 3.678 & 348.56 & 1.587 & 0.9999 & 0.838 & 2297.6 & 4950 & 176.75 & 0.00099 & 0.010945 & 1021 & 148 \\
\hline 2.931 & 348.56 & 1.588 & 0.811 & 0.527 & 2290.22 & 4950 & 173.52 & 0.001 & 0.010855 & 1035 & 147 \\
\hline 5.597 & 394.91 & 1.587 & 0.9999 & 0.842 & 1935.37 & 4950 & 191.55 & 0.00095 & 0.01358 & 1098 & 142 \\
\hline 4.571 & 394.91 & 1.588 & 0.813 & 0.508 & 1923.55 & 4950 & 186.22 & 0.00096 & 0.013325 & 1141 & 140 \\
\hline 5.295 & 402.26 & 1.587 & 0.9999 & 0.844 & 2104.08 & 4950 & 185.29 & 0.00097 & 0.0123 & 1055 & 144 \\
\hline 4.227 & 402.26 & 1.588 & 0.815 & 0.514 & 2093.04 & 4950 & 180.34 & 0.00098 & 0.012085 & 1090 & 144 \\
\hline 4.958 & 409 & 1.587 & 0.9999 & 0.838 & 2264.82 & 4950 & 178.45 & 0.000985 & 0.01119 & 1024 & 147 \\
\hline 3.868 & 409 & 1.588 & 0.809 & 0.513 & 2254.57 & 4950 & 174.8 & 0.000995 & 0.011065 & 1045 & 147 \\
\hline 1.378 & 256.03 & 1.587 & 0.464 & 0.183 & 1771.87 & 4950 & 193.27 & 0.00095 & 0.01472 & 1190 & 137 \\
\hline 0.57 & 256.03 & 1.588 & 0.163 & 0.064 & 1770.37 & 4950 & 194.44 & 0.00095 & 0.01481 & 1190 & 137 \\
\hline 2.098 & 319.87 & 1.587 & 0.513 & 0.253 & 1860.55 & 4950 & 189.8 & 0.00096 & 0.01392 & 1160 & 139 \\
\hline 0.849 & 319.87 & 1.588 & 0.233 & 0.1 & 1857.6 & 4950 & 190.98 & 0.00096 & 0.014005 & 1160 & 39 \\
\hline 2.572 & 374.66 & 1.587 & 0.511 & 0.254 & 1993.5 & 4950 & 185.18 & 0.00097 & 0.012865 & 1117 & 142 \\
\hline
\end{tabular}

\section{R134a}

\begin{tabular}{|c|c|c|c|c|c|c|c|c|c|c|c|}
\hline 1.141 & 374.66 & 1.588 & 0.235 & 0.132 & 1989.77 & 4067 & 186.36 & 0.00097 & 0.01295 & 1118 & 142 \\
\hline 4.477 & 277.04 & 1.588 & 0.881 & 0.604 & 1027.45 & 4067 & 162.92 & 0.00087 & 0.01983 & 1770 & 131 \\
\hline 4.382 & 310.29 & 1.588 & 0.868 & 0.576 & 1209.26 & 4067 & 156.08 & 0.000895 & 0.01665 & 1641 & 136 \\
\hline 4.418 & 315.35 & 1.588 & 0.844 & 0.512 & 1207.27 & 4067 & 156.16 & 0.00089 & 0.016685 & 1642 & 136 \\
\hline 6.299 & 388.94 & 1.588 & 0.896 & 0.614 & 1302.2 & 4067 & 152.76 & 0.00091 & 0.01537 & 1583 & 138 \\
\hline 6.532 & 391.49 & 1.588 & 0.886 & 0.589 & 1265.27 & 4067 & 154.1 & 0.0009 & 0.01587 & 1606 & 137 \\
\hline 7.004 & 350.96 & 1.587 & 0.97 & 0.793 & 986.91 & 4067 & 164.57 & 0.00087 & 0.02073 & 1803 & 130 \\
\hline 6.269 & 350.96 & 1.588 & 0.766 & 0.479 & 970.82 & 4067 & 165.2 & 0.00087 & 0.021085 & 1815 & 130 \\
\hline 7.365 & 348.86 & 1.587 & 0.964 & 0.781 & 942.11 & 4067 & 166.36 & 0.00086 & 0.021775 & 1841 & 129 \\
\hline 6.553 & 348.86 & 1.588 & 0.754 & 0.459 & 925.24 & 4067 & 167.02 & 0.00086 & 0.022185 & 1855 & 128 \\
\hline 9.801 & 469.51 & $\begin{array}{l}1.500 \\
1.587\end{array}$ & 0.9999 & $\begin{array}{l}0.439 \\
0.839\end{array}$ & $\begin{array}{l}1285.79 \\
12859\end{array}$ & 4067 & $\begin{array}{l}153.66 \\
\end{array}$ & 0.0009 & 0.015645 & 1591 & $\begin{array}{l}120 \\
137\end{array}$ \\
\hline 8.279 & 469.51 & 1.588 & 0.814 & 0.528 & 1264.75 & 4067 & 154.16 & 0.0009 & 0.01589 & 1608 & 137 \\
\hline 4.865 & 270.02 & 1.587 & 0.948 & 0.749 & 989.36 & 4067 & 164.41 & 0.00087 & 0.02064 & 1800 & 130 \\
\hline 3.793 & 270.02 & 1.588 & 0.72 & 0.415 & 979.76 & 4067 & 164.77 & 0.00087 & 0.02084 & 1807 & 130 \\
\hline 3.898 & 290.21 & 1.587 & 0.946 & 0.751 & 1373.11 & 4067 & 150.16 & 0.00091 & 0.01447 & 1541 & 140 \\
\hline 2.751 & 290.21 & 1.588 & 0.722 & 0.422 & $\begin{array}{l}1365.67 \\
\end{array}$ & 4067 & $\begin{array}{l}150.4 \\
\text { S }\end{array}$ & 0.00091 & 0.014545 & 1545 & 139 \\
\hline 3.64 & 232.44 & 1.587 & 0.915 & 0.651 & 998.36 & 4067 & 164.02 & 0.00087 & 0.02042 & 1791 & 130 \\
\hline 2.076 & 232.44 & 1.588 & 0.613 & 0.27 & 991.74 & 4067 & 164.25 & 0.00087 & 0.020555 & 1796 & 130 \\
\hline 1.177 & 135.71 & 1.587 & 0.916 & 0.683 & 1131.19 & 4067 & 158.88 & 0.00089 & 0.01785 & 1692 & 134 \\
\hline 0.646 & 135.71 & 1.588 & 0.648 & 0.299 & 1129.54 & 4067 & 158.94 & 0.000885 & 0.017875 & 1692 & 134 \\
\hline 0.879 & 120.62 & 1.587 & 0.9999 & 0.931 & 1026.66 & 4067 & 164.79 & 0.00087 & 0.02004 & 1730 & 132 \\
\hline 0.86 & 120.62 & 1.588 & 0.911 & 0.637 & 1025.3 & 4067 & 162.91 & 0.00087 & 0.01982 & 1769 & 131 \\
\hline 0.694 & 113.3 & 1.588 & 0.901 & 0.615 & 1014.37 & 4067 & 163.32 & 0.00087 & 0.020045 & 1778 & 131 \\
\hline $\begin{array}{l}10.954 \\
\end{array}$ & 438.38 & 1.587 & 0.968 & 0.764 & 1037.43 & 4067 & $\begin{array}{l}162.7 \\
\text { S }\end{array}$ & 0.00087 & 0.01971 & 1765 & 132 \\
\hline 8.421 & 438.38 & 1.588 & 0.733 & 0.417 & 1014.25 & 4067 & 163.56 & 0.00087 & 0.02017 & 1782 & 131 \\
\hline $\begin{array}{l}0.42 .612 \\
12.12\end{array}$ & 458.82 & 1.587 & 0.967 & 0.777 & 986.08 & 4067 & 164.75 & 0.00087 & 0.020835 & 1806 & 130 \\
\hline 10.059 & 458.82 & 1.588 & 0.75 & 0.451 & 959.1 & 4067 & 165.77 & 0.000865 & 0.02143 & 1828 & 130 \\
\hline 7.17 & $\begin{array}{l}450.02 \\
459.87\end{array}$ & $\begin{array}{l}1.580 \\
1.588\end{array}$ & 0.723 & 0.394 & 1182.24 & 4067 & $\begin{array}{l}157.16 \\
\end{array}$ & 0.00089 & $\begin{array}{l}0.02171 \\
0.0171\end{array}$ & $\begin{array}{l}1020 \\
1660\end{array}$ & 135 \\
\hline 7.276 & 465.08 & 1.588 & 0.762 & 0.461 & 1289.16 & 4067 & 153.26 & 0.0009 & 0.01555 & 1592 & 137 \\
\hline 7.086 & 392.77 & 1.587 & 0.995 & 0.81 & 1301.66 & 4067 & $\begin{array}{l}152.79 \\
\end{array}$ & 0.000905 & 0.015385 & 1584 & 138 \\
\hline 5.564 & 392.77 & 1.588 & 0.781 & 0.478 & 1287.02 & 4067 & 153.29 & 0.0009 & 0.015565 & 1593 & 137 \\
\hline 7.836 & 385.9 & $\begin{array}{l}1.580 \\
\end{array}$ & 0.986 & 0.792 & 1135.83 & 4067 & 158.88 & 0.000885 & 0.01785 & 1692 & 134 \\
\hline 6.421 & 385.9 & 1.588 & 0.763 & 0.454 & 1119.38 & 4067 & 159.48 & 0.00088 & 0.018125 & 1703 & 133 \\
\hline $\begin{array}{l}7.4787 \\
7.78\end{array}$ & 364.66 & $\begin{array}{l}1.580 \\
1.587\end{array}$ & 0.97 & 0.782 & 1039.37 & 4067 & $\begin{array}{l}162.54 \\
164\end{array}$ & 0.000875 & 0.01963 & 1762 & 132 \\
\hline 6.475 & 364.66 & 1.588 & 0.753 & 0.452 & 1023.05 & 4067 & 163.15 & 0.00087 & 0.019955 & 1773 & 131 \\
\hline 3.963 & 253.66 & $\begin{array}{l}1.587 \\
\text {. }\end{array}$ & 0.948 & 0.761 & 1063.84 & 4067 & $\begin{array}{l}161.5 \\
161.5\end{array}$ & 0.00088 & $\begin{array}{l}0.0191 \\
0\end{array}$ & 1741 & 132 \\
\hline 3.13 & 253.66 & 1.588 & 0.732 & 0.438 & 1056.07 & 4067 & 161.79 & 0.00088 & 0.01924 & 1747 & 132 \\
\hline 1.554 & $\begin{array}{l}163.35 \\
1635\end{array}$ & 1.587 & 0.984 & $\begin{array}{l}0.458 \\
0.87\end{array}$ & 1089.99 & 4067 & 160.45 & 0.00088 & 0.01858 & 1721 & 133 \\
\hline 1.467 & 163.35 & 1.588 & 0.848 & 0.568 & 1087.16 & 4067 & 160.55 & 0.00088 & 0.018635 & 1723 & 133 \\
\hline 1.262 & 149.9 & 1.587 & 0.946 & 0.755 & 1265.69 & 4067 & 153.94 & 0.0009 & 0.01581 & 1603 & 137 \\
\hline 0.743 & 149.9 & 1.588 & 0.724 & 0.398 & 1263.8 & 4067 & 154 & 0.0009 & 0.01583 & 1604 & 137 \\
\hline 3.819 & 408.11 & 1.587 & 0.474 & 0.21 & 1069.74 & 4067 & 161.26 & 0.00088 & 0.01898 & 1736 & 132 \\
\hline 1.559 & 408.11 & 1.588 & 0.192 & 0.104 & 1063.91 & 4067 & 161.45 & 0.00088 & 0.01907 & 1740 & 132 \\
\hline 3.826 & 412.14 & 1.587 & 0.471 & 0.204 & 1075.96 & 4067 & 161.03 & 0.00088 & 0.018865 & 1732 & 132 \\
\hline 1.498 & 412.14 & 1.588 & 0.186 & 0.096 & 1070.13 & 4067 & $\begin{array}{l}161.21 \\
\end{array}$ & 0.00088 & 0.01895 & 1735 & 132 \\
\hline 1.175 & 219.99 & 1.587 & 0.488 & 0.231 & 1203.88 & 4067 & 156.19 & 0.00089 & 0.01669 & 1642 & 136 \\
\hline 0.646 & 163.28 & 1.587 & 0.472 & 0.202 & 1191.48 & 4067 & 156.63 & 0.00089 & 0.016875 & 1651 & 135 \\
\hline 0.702 & 159.65 & 1.587 & 0.492 & 0.25 & 1141.44 & 4067 & 158.49 & 0.00089 & 0.017675 & 1684 & 134 \\
\hline 1.659 & 263.95 & 1.587 & 0.468 & 0.188 & 1040.05 & 4067 & 162.35 & 0.00088 & 0.01953 & 1758 & 132 \\
\hline 0.644 & 263.95 & 1.588 & 0.168 & 0.079 & 1038.07 & 4067 & 162.41 & 0.00088 & 0.019565 & 1759 & 132 \\
\hline 1.595 & 233.13 & 1.587 & 0.501 & 0.246 & 1100.07 & 4067 & $\begin{array}{l}160.06 \\
\end{array}$ & 0.00088 & 0.018395 & 1713 & 133 \\
\hline 0.592 & 233.13 & 1.588 & 0.227 & 0.127 & 1098.09 & 4067 & 160.12 & 0.00088 & 0.018425 & 1714 & 133 \\
\hline 4.029 & 411.39 & 1.587 & 0.496 & 0.22 & 1085.52 & 4067 & 160.67 & 0.00088 & 0.018695 & 1725 & 133 \\
\hline 1.734 & 411.39 & 1.588 & 0.2 & 0.102 & 1079.25 & 4067 & 160.87 & 0.00088 & 0.018785 & 1729 & 132 \\
\hline 3.603 & 403.22 & $\begin{array}{l}1.500 \\
1.587\end{array}$ & $\begin{array}{l}0.502 \\
0.52\end{array}$ & 0.239 & 1210.01 & 4067 & $\begin{array}{l}156.02 \\
15.02\end{array}$ & 0.000895 & 0.01662 & 1639 & 136 \\
\hline 1.61 & 403.22 & 1.588 & 0.221 & 0.124 & 1204.39 & 4067 & 156.19 & 0.00089 & 0.016695 & 1642 & 136 \\
\hline $\begin{array}{l}4.01 \\
4.37\end{array}$ & $\begin{array}{l}403.22 \\
460.74\end{array}$ & $\begin{array}{l}1.580 \\
1.587\end{array}$ & 0.497 & 0.2 & 1274.29 & 4067 & 153 & 0.0009 & $\begin{array}{l}0.01572 \\
0\end{array}$ & $\begin{array}{l}1599 \\
1599\end{array}$ & 137 \\
\hline 2.298 & 460.74 & 1.588 & 0.233 & 0.143 & 1266.97 & 4067 & 153.93 & 0.0009 & 0.0158 & 1603 & 137 \\
\hline 4.694 & 467.09 & 1.587 & 0.492 & 0.225 & 1147.6 & 4067 & $\begin{array}{l}158.35 \\
\end{array}$ & 0.00089 & 0.017615 & 1682 & 134 \\
\hline 2.104 & 467.09 & 1.588 & 0.207 & 0.111 & 1139.92 & 4067 & 158.59 & 0.00089 & 0.01772 & 1686 & 134 \\
\hline 5.258 & 461.86 & $\begin{array}{l}1.580 \\
1.587\end{array}$ & 0.482 & $\begin{array}{l}0.1 \\
0.2\end{array}$ & 1001.26 & 4067 & 163.94 & 0.00087 & 0.02038 & $\begin{array}{l}1780 \\
1789\end{array}$ & $\begin{array}{l}130 \\
130\end{array}$ \\
\hline 2.194 & 461.86 & 1.588 & 0.196 & 0.099 & 992.75 & 4067 & 164.22 & 0.00087 & 0.02053 & 1795 & 130 \\
\hline 2.886 & 322.01 & $\begin{array}{l}1.580 \\
1.587\end{array}$ & 0.493 & 0.226 & 1033.1 & 4067 & $\begin{array}{l}162.62 \\
162.65\end{array}$ & 0.00087 & 0.019685 & 1764 & 131 \\
\hline 1.163 & 322.01 & 1.588 & 0.206 & 0.105 & 1028.96 & 4067 & 162 & 0.00087 & 0.019755 & 1767 & 131 \\
\hline $\begin{array}{l}3.1115 \\
3.115\end{array}$ & 324.8 & $\begin{array}{l}1.587 \\
\text {. }\end{array}$ & 0.484 & 0.21 & 934.99 & 4067 & $\begin{array}{l}102.10 \\
166.51\end{array}$ & 0.00086 & 0.02187 & 1844 & $\begin{array}{l}129 \\
129\end{array}$ \\
\hline 2.711 & 324.62 & 1.587 & 0.517 & 0.276 & 1214.65 & 4067 & 155.83 & 0.0009 & 0.016545 & 1636 & 136 \\
\hline 1.336 & 324.62 & 1.588 & 0.258 & 0.164 & 1210.57 & 4067 & 155.95 & 0.0009 & 0.016595 & 1638 & 136 \\
\hline 2.599 & 334.11 & & 0.517 & 0.27 & 1369.91 & 4067 & 150.23 & 0.00091 & 0.0145 & 1542 & 39 \\
\hline
\end{tabular}




$\begin{array}{llllllllllll}1.193 & 334.11 & 1.588 & 0.252 & 0.158 & 1366.04 & 4067 & 150.35 & 0.00091 & 0.01453 & 1544 & 139 \\ 3.189 & 346.82 & 1.587 & 0.501 & 0.231 & 1080.24 & 4067 & 160.85 & 0.00088 & 0.018775 & 1729 & 132 \\ 1.328 & 346.82 & 1.588 & 0.211 & 0.111 & 1075.41 & 4067 & 161 & 0.00088 & 0.01885 & 1731 & 132 \\ 0.808 & 162 & 1.587 & 0.48 & 0.209 & 934.12 & 4067 & 166.49 & 0.00086 & 0.02186 & 1843 & 129 \\ 0.859 & 160.47 & 1.587 & 0.491 & 0.236 & 1030 & 4067 & 162.72 & 0.00087 & 0.019725 & 1765 & 131 \\ 0.749 & 164.75 & 1.587 & 0.515 & 0.255 & 1320.61 & 4067 & 151.95 & 0.00091 & 0.015085 & 1570 & 138 \\ 1.762 & 268.43 & 1.587 & 0.496 & 0.235 & 1233.95 & 4067 & 155.1 & 0.0009 & 0.016255 & 1623 & 136 \\ 0.65 & 268.43 & 1.588 & 0.217 & 0.127 & 1231.78 & 4067 & 155.16 & 0.0009 & 0.01628 & 1625 & 136 \\ 1.739 & 268.28 & 1.587 & 0.496 & 0.234 & 1232.45 & 4067 & 155.15 & 0.0009 & 0.016275 & 1625 & 136 \\ 0.666 & 268.28 & 1.588 & 0.216 & 0.126 & 1230.25 & 4067 & 155.22 & 0.0009 & 0.016305 & 1626 & 136\end{array}$




\section{APPENDIX B}

\section{Evaporation pressure drop of NIST in micro-fin tube(file: nist_eva.in)}

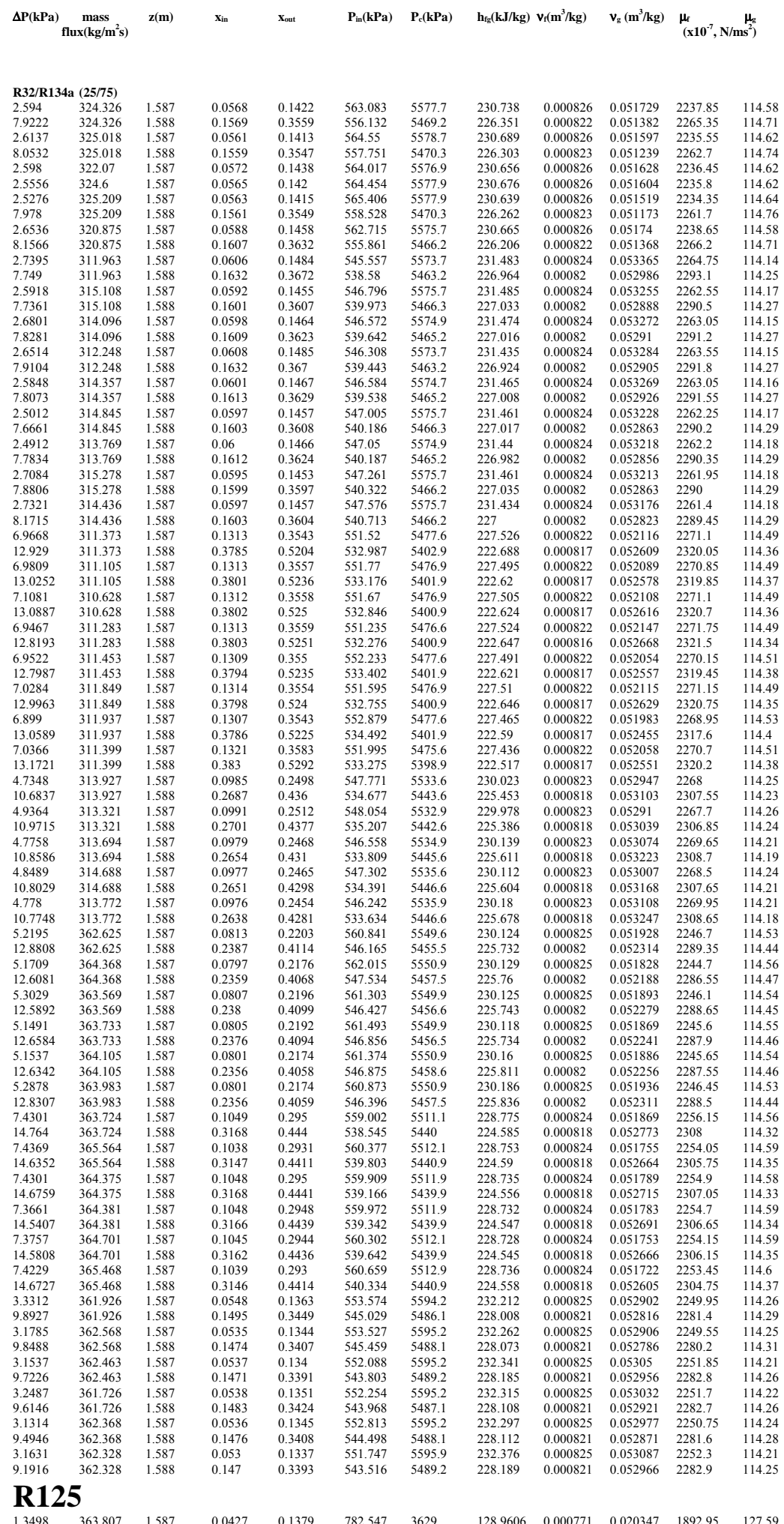




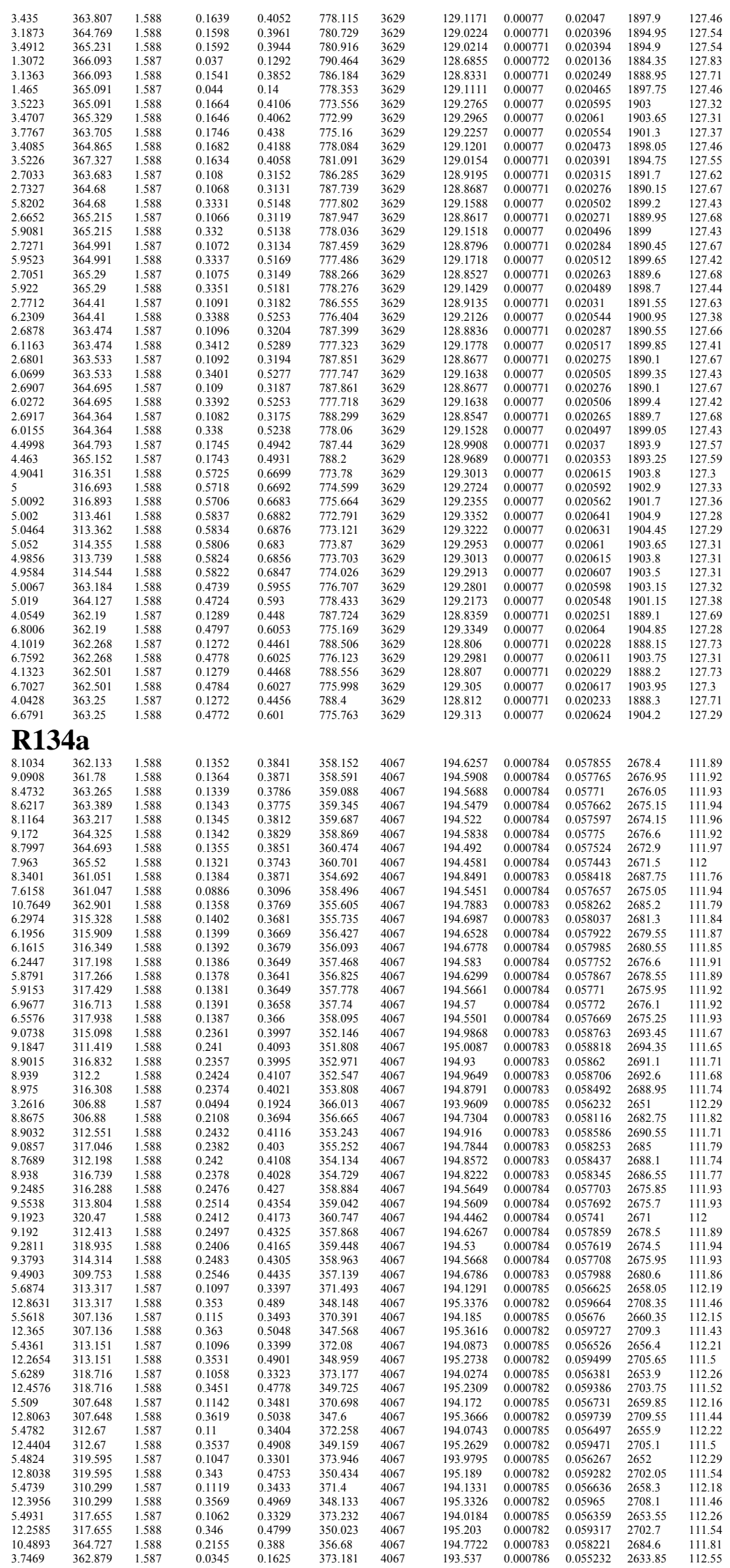




\begin{tabular}{|c|c|c|c|c|c|c|c|c|c|c|c|}
\hline 7 & 3 & 1.588 & 07 & 18 & 263 & 4067 & 19 & 0. & 07 & 1.05 & 99 \\
\hline 10.8475 & 364.893 & 1.588 & 0.2196 & 0.3982 & 359.98 & 4067 & 194.5617 & 0.000784 & 0.057693 & 2675.75 & 111.93 \\
\hline 11.1095 & 361.8 & 1.588 & 0.2238 & 0.4065 & 357.619 & 4067 & 194.7183 & 0.000783 & 0.058084 & 2682.3 & 111.83 \\
\hline 10.7649 & 361.852 & 1.588 & 0.2234 & 0.4062 & 357.408 & 4067 & 194.7293 & 0.000783 & 0.058114 & 2682.8 & 111.82 \\
\hline 10.8505 & 361.351 & 1.588 & 0.2249 & 0.408 & 357.164 & 4067 & 194.7432 & 0.000783 & 0.058146 & 2683.35 & 111.82 \\
\hline 10.6628 & 362.459 & 1.588 & 0.221 & 0.4009 & 356.362 & 4067 & 194.7961 & 0.000783 & 0.05828 & 2685.55 & 111.79 \\
\hline 11.2227 & 360.774 & 1.588 & 0.2239 & 0.4061 & 356.792 & 4067 & 194.7682 & 0.000783 & 0.058212 & 2684.45 & 111.81 \\
\hline 10.9766 & 361.904 & 1.588 & 0.2247 & 0.406 & 357.036 & 4067 & 194.7632 & 0.000783 & 0.058195 & 2684.2 & 111.81 \\
\hline 11.1615 & 362.414 & 1.588 & 0.2234 & 0.4048 & 357.753 & 4067 & 194.7113 & 0.000783 & 0.058067 & 2682.05 & 111.84 \\
\hline 6.1132 & 363.365 & 1.587 & 0.0847 & 0.294 & 380.543 & 4067 & 193.6481 & 0.000786 & 0.055479 & 2638.6 & 112.48 \\
\hline 14.7093 & 363.365 & 1.588 & 0.3087 & 0.4565 & 354.118 & 4067 & 195.0313 & 0.000783 & 0.058874 & 2695.4 & 111.64 \\
\hline 6.0113 & 363.054 & 1.587 & 0.0852 & 0.2954 & 380.809 & 4067 & 193.6263 & 0.000786 & 0.055426 & 2637.7 & 112.5 \\
\hline 14.6505 & 363.054 & 1.588 & 0.3101 & 0.4589 & 354.608 & 4067 & 194.9954 & 0.000783 & 0.058784 & 2694 & 111.66 \\
\hline 5.9975 & 360.953 & 1.587 & 0.0864 & 0.2986 & 380.288 & 4067 & 193.6681 & 0.000786 & 0.055525 & 2639.4 & 112.46 \\
\hline 14.4862 & 360.953 & 1.588 & 0.3137 & 0.4652 & 353.758 & 4067 & 195.0542 & 0.000782 & 0.058931 & 2696.4 & 111.63 \\
\hline 6.0168 & 360.87 & 1.587 & 0.08 & 0.2992 & 380 & 4067 & 193.6 & 0.000786 & 0.055446 & 2638.05 & 112.49 \\
\hline 14.5186 & 360.87 & 1.588 & 0.3143 & 0.4664 & 354.306 & 4067 & 195.0173 & 0.000783 & 0.058836 & 2694.85 & 111.65 \\
\hline 6.0921 & 359.803 & 1.587 & 0.0566 & 0.2563 & 381.329 & 4067 & 193.1616 & 0.000787 & 0.054361 & 2618.85 & 112.78 \\
\hline $\begin{array}{l}14.617 \\
0.07\end{array}$ & 359.803 & 1.588 & 0.2805 & 0.4223 & 362.495 & 4067 & 194.608 & 0.000784 & 0.057799 & 2677.9 & 111.9 \\
\hline 6.0115 & 361.519 & 1.587 & 0.0867 & 0.2997 & 381.476 & 4067 & 193.5963 & 0.000786 & 0.055356 & 2636.5 & 112.51 \\
\hline 14.6441 & 361.519 & 1.588 & 0.3148 & 0.4671 & 354.898 & 4067 & 194.9824 & 0.000783 & 0.058748 & 2693.35 & 111.67 \\
\hline 6.0885 & 363.239 & 1.587 & 0.0856 & 0.2975 & 382.431 & 4067 & 193.5345 & 0.000787 & 0.055211 & 2633.95 & 112.55 \\
\hline 14.4741 & 363.239 & 1.588 & 0.3124 & 0.4632 & 355.954 & 4067 & 194.9106 & 0.000783 & 0.058567 & 2690.35 & 111.71 \\
\hline 5.9509 & 363.302 & 1.587 & 0.0859 & 0.2971 & 382.433 & 406 & 193.5216 & 0.000787 & 0.055182 & 2633.45 & 112.56 \\
\hline $\begin{array}{l}14.6539 \\
\end{array}$ & 363.302 & 1.58 & 0.31 & 0.4 & 356.329 & 4067 & 194.8896 & 0.000783 & 0.058512 & 2689.45 & 111.73 \\
\hline 5.9466 & 361.473 & 1.587 & 0.0864 & 0.2989 & 381.51 & 4067 & 193.5854 & 0.000786 & 0.055328 & 2636 & 112.52 \\
\hline 14.6805 & 361.473 & 1.588 & 0.314 & 0.466 & 355.224 & 4067 & 194.9574 & 0.000783 & 0.058687 & 2692.35 & 111.69 \\
\hline & 3.657 & 1.587 & 0.0852 & 2969 & 665 & 4067 & 93.5284 & 0.000787 & 0.05519 & 2633.75 & 112.56 \\
\hline & & & & & & & & 0. & & & \\
\hline
\end{tabular}

R22

\begin{tabular}{|c|c|c|c|c|c|c|c|c|c|c|c|}
\hline 4.0601 & 361.861 & 1.588 & 0.0898 & 0.2353 & 575.824 & 4990 & 8.8643 & 0.00079 & 0.040705 & 2102.75 & 119.43 \\
\hline 9966 & 363.208 & 1.588 & 0.0891 & 0.2339 & 575.52 & 4990 & 198.8803 & 0.00079 & 0.040728 & 2103.1 & 119.42 \\
\hline 4.3924 & 364.035 & 1.588 & 0.1221 & 0.2794 & 579.533 & 4990 & 198.7088 & 0.00079 & 0.040466 & 2098.9 & 119.52 \\
\hline 4.3124 & 364.332 & 1.588 & 0.122 & 0.2794 & 579.793 & 4990 & 198.6948 & 0.00079 & 0.040445 & 2098.55 & 119.54 \\
\hline 7.0973 & 360.729 & 1.588 & 0.236 & 0.3695 & 579.98 & 4990 & 198.7425 & 0.00079 & 0.04052 & 2099.7 & 119.51 \\
\hline 7.1483 & 361.663 & 1.588 & 0.2353 & 0.3689 & 581.622 & 4990 & 198.6677 & 0.00079 & 0.040405 & 2097.9 & 119.56 \\
\hline 7.3091 & 362.178 & 1.588 & 0.2352 & 0.3688 & 581.976 & 4990 & 198.6528 & 0.00079 & 0.040383 & 2097.5 & 119.56 \\
\hline 7.9197 & 364.693 & 1.588 & 0.2448 & 0.3906 & 573.969 & 4990 & 199.0297 & 0.000789 & 0.040958 & 2106.8 & 119.32 \\
\hline 7.9958 & 365 & 1.588 & 0.2443 & 0.39 & 574.018 & 4990 & 199.0327 & 0.000789 & 0.040963 & 2106.85 & 119.32 \\
\hline 7.6224 & 365.718 & 1.588 & 0.2434 & 0.3887 & 573.98 & 4990 & 199.0277 & 0.000789 & 0.040956 & 2106.7 & 119.32 \\
\hline 7.8519 & 366.376 & 1.588 & 0.2418 & 0.3862 & 572.854 & 4990 & 199.0796 & 0.000789 & 0.041037 & 2108 & 119.28 \\
\hline 3.4404 & 367.858 & 1.587 & 0.0561 & 0.1906 & 586.694 & 4990 & 198.3846 & 0.000791 & 0.039978 & 2090.95 & 119.74 \\
\hline 7.7211 & 367.858 & 1.588 & 0.2068 & 0.343 & 577.468 & 4990 & 198.901 & 0.00079 & 0.040761 & 2103.6 & 119.4 \\
\hline 3.7581 & 361.031 & 1.587 & 0.0622 & 0.2155 & 593.619 & 4990 & 198.0843 & 0.000792 & 0.039535 & 2083.65 & 119.92 \\
\hline 9.1305 & 361.031 & 1.588 & 0.2349 & 0.4153 & 583.445 & 4990 & 198.6635 & 0.00079 & 0.040398 & 2097.8 & 119.56 \\
\hline 3.7574 & 361.804 & 1.587 & 0.0615 & 0.2144 & 594.492 & 4990 & 198.0445 & 0.000792 & 0.039476 & 2082.7 & 119.94 \\
\hline 9.1302 & 361.804 & 1.588 & 0.2337 & 0.4133 & 584.421 & 4990 & 198.6176 & 0.00079 & 0.040328 & 2096.65 & 119.58 \\
\hline 3.6755 & 362.089 & 1.587 & 0.0618 & 0.2145 & 594.501 & 4990 & 198.0395 & 0.000792 & 0.039469 & 2082.55 & 119.95 \\
\hline 8.9592 & 362.089 & 1.588 & 0.2336 & 0.4134 & 584.725 & 4990 & 198.5957 & 0.00079 & 0.040294 & 2096.1 & 119.6 \\
\hline 3.5842 & 363.58 & 1.587 & 0.0604 & 0.212 & 595.956 & 4990 & 197.9786 & 0.000792 & 0.039379 & 2081.1 & 119.99 \\
\hline 8.9774 & 363.58 & 1.588 & 0.2312 & 0.4093 & 585.821 & 4990 & 198.5568 & 0.00079 & 0.040235 & 2095.15 & 119.62 \\
\hline 3.6028 & 363.598 & 1.587 & 0.061 & 0.2131 & 595.664 & 4990 & 197.9926 & 0.000792 & 0.0394 & 2081.45 & 119.98 \\
\hline 8.9375 & 363.598 & 1.588 & 0.2324 & 0.411 & 585.383 & 4990 & 198.5767 & 0.00079 & 0.040267 & 2095.65 & 119.61 \\
\hline 3.6146 & 364.456 & 1.587 & 0.0604 & 0.2121 & 596.55 & 4990 & 197.9497 & 0.000792 & 0.039338 & 2080.4 & 120.01 \\
\hline 8.8297 & 364.456 & 1.588 & 0.2312 & 0.4089 & 586.583 & 4990 & 198.5149 & 0.000791 & 0.040173 & 2094.15 & 119.65 \\
\hline 5.7453 & 366.099 & 1.587 & 0.0923 & 0.3225 & 596.033 & 4990 & 198.0552 & 0.000792 & 0.039493 & 2083 & 119.94 \\
\hline 12.2161 & 366.099 & 1.588 & 0.3474 & 0.5003 & 579.159 & 4990 & 198.9635 & 0.000789 & 0.040855 & 2105.25 & 119.36 \\
\hline 5.798 & 367.033 & 1.587 & 0.0918 & 0.3211 & 596.36 & 4990 & 198.0403 & 0.000792 & 0.03947 & 2082.65 & 119.95 \\
\hline 12.1783 & 367.033 & 1.588 & 0.346 & 0.498 & 579.639 & 4990 & 198.9385 & 0.00079 & 0.040816 & 2104.55 & 119.38 \\
\hline 5.7508 & 366.813 & 1.587 & 0.0922 & 0.3216 & 596.006 & 4990 & 198.0592 & 0.000792 & 0.039497 & 2083.1 & 119.93 \\
\hline 12.1587 & 366.813 & 1.588 & 0.3464 & 0.4986 & 579.009 & 4990 & 198.9714 & 0.000789 & 0.040866 & 2105.4 & 119.35 \\
\hline 5.838 & 367.449 & 1.587 & 0.0915 & 0.3197 & 596.091 & 4990 & 198.0532 & 0.000792 & 0.039491 & 2083 & 119.94 \\
\hline 12.0352 & 367.449 & 1.588 & 0.3443 & 0.4958 & 579.276 & 4990 & 198.9525 & 0.000789 & 0.040837 & 2104.9 & 119.37 \\
\hline 5.7648 & 367.409 & 1.587 & 0.0918 & 0.32 & 595.531 & 4990 & 198.0791 & 0.000792 & 0.039529 & 2083.6 & 119.93 \\
\hline 12.1569 & 367.409 & 1.588 & 0.3446 & 0.4961 & 578.509 & 4990 & 198.9933 & 0.000789 & 0.040901 & 2105.9 & 119.35 \\
\hline 5.9534 & 368.411 & 1.587 & 0.0911 & 0.3187 & 596.033 & 4990 & 198.0581 & 0.000792 & 0.039497 & 2083.05 & 119.94 \\
\hline 12.2778 & 368.411 & 1.588 & 0.3433 & 0.494 & 579.053 & 4990 & 198.9675 & 0.000789 & 0.04086 & 2105.3 & 119.36 \\
\hline 5.7865 & 368.507 & 1.587 & 0.0913 & 0.3184 & 595.651 & 4990 & 198.0731 & 0.000792 & 0.039519 & 2083.4 & 119.93 \\
\hline 12.6853 & 368.507 & 1.588 & 0.343 & 0.4937 & 578.696 & 4990 & 198.9923 & 0.000789 & 0.040899 & 2105.85 & 119.35 \\
\hline 5.8214 & 369.334 & 1.587 & 0.0907 & 0.317 & 595.623 & 4990 & 198.0751 & 0.000792 & 0.039521 & 2083.5 & 119.93 \\
\hline 12.354 & 369.334 & 1.588 & 0.3415 & 0.4915 & 578.657 & 4990 & 198.9874 & 0.000789 & 0.040891 & 2105.8 & 119.35 \\
\hline 5.6551 & 369.452 & 1.587 & 0.091 & 0.3173 & 595.395 & 4990 & 198.0841 & 0.000792 & 0.039535 & 2083.7 & 119.92 \\
\hline 12.3517 & 369.452 & 1.588 & 0.3419 & 0.4919 & 578.353 & 4990 & 199.0053 & 0.000789 & 0.040918 & 2106.25 & 119.33 \\
\hline 5.7931 & 370.179 & 1.587 & 0.0905 & 0.3173 & 596.207 & 4990 & 198.0502 & 0.000792 & 0.039484 & 2082.85 & 119.94 \\
\hline 12.3387 & 370.179 & 1.588 & 0.342 & 0.4917 & 579.202 & 4990 & 198.9664 & 0.000789 & 0.040859 & 2105.25 & 119.36 \\
\hline 1.7432 & 311.501 & 1.587 & 0.038 & 0.1208 & 586.836 & 4990 & 198.3149 & 0.000791 & 0.039875 & 2089.25 & 119.77 \\
\hline 5.1639 & 311.501 & 1.588 & 0.1356 & 0.3587 & 582.715 & 4990 & 198.576 & 0.00079 & 0.040267 & 2095.65 & 119.61 \\
\hline 4.9303 & 311.83 & 1.588 & 0.1356 & 0.359 & 583.725 & 4990 & 198.5252 & 0.000791 & 0.040191 & 2094.4 & 119.64 \\
\hline 1.5084 & 312.588 & 1.587 & 0.0376 & 0.1197 & 588.338 & 4990 & 198.2421 & 0.000791 & 0.039769 & 2087.5 & 119.82 \\
\hline 4.6848 & 312.588 & 1.588 & 0.134 & 0.3564 & 584.505 & 4990 & 198.4883 & 0.000791 & 0.040136 & 2093.5 & 119.67 \\
\hline 4.9028 & 314.177 & 1.588 & 0.132 & 0.3519 & 586.19 & 4990 & 198.4115 & 0.000791 & 0.040021 & 2091.65 & 119.71 \\
\hline 1.5691 & 314.122 & 1.587 & 0.0373 & 0.1187 & 589.745 & 4990 & 198.1793 & 0.000791 & 0.039676 & 2086 & 119.86 \\
\hline 5.0711 & 314.122 & 1.588 & 0.1331 & 0.3526 & 585.868 & 4990 & 198.4305 & 0.000791 & 0.040048 & 2092.1 & 119.7 \\
\hline 1.5031 & 314.41 & 1.587 & 0.0361 & 0.1169 & 591.534 & 4990 & 198.0995 & 0.000792 & 0.039558 & 2084 & 119.91 \\
\hline 4.8052 & 314.41 & 1.588 & 0.1313 & 0.349 & 587.632 & 4990 & 198.3506 & 0.000791 & 0.039929 & 2090.15 & 119.75 \\
\hline 4.7907 & 315.492 & 1.588 & 0.1308 & 0.3482 & 587.325 & 4990 & 198.3616 & 0.000791 & 0.039945 & 2090.4 & 119.74 \\
\hline 4.8995 & 315.703 & 1.588 & 0.1303 & 0.3466 & 588.492 & 4990 & 198.3138 & 0.000791 & 0.039874 & 2089.2 & 119.78 \\
\hline 1.4918 & 316.167 & 1.587 & 0.0361 & 0.116 & 591.68 & 4990 & 198.0925 & 0.000792 & 0.039548 & 2083.9 & 119.91 \\
\hline 4.8098 & 316.167 & 1.588 & 0.1299 & 0.3451 & 587.746 & 4990 & 198.3456 & 0.000791 & 0.039923 & 2090.05 & 119.76 \\
\hline 4.7884 & 316.578 & 1.588 & 0.1293 & 0.3434 & 588.427 & 4990 & 198.3118 & 0.000791 & 0.039872 & 2089.2 & 119.78 \\
\hline 3.176 & 312.2 & 1.587 & 0.0779 & 0.2441 & 592.335 & 4990 & 198.1243 & 0.000792 & 0.039595 & 2084.65 & 119.9 \\
\hline 7.3726 & 312.2 & 1.588 & 0.2644 & 0.4439 & 583.562 & 4990 & 198.6158 & 0.00079 & 0.040325 & 2096.55 & 119.58 \\
\hline 3.0567 & 312.435 & 1.587 & 0.0777 & 0.2431 & 592.104 & 4990 & 198.1313 & 0.000792 & 0.039604 & 2084.8 & 119.89 \\
\hline 7.3991 & 312.435 & 1.588 & 0.2633 & 0.4417 & 583.563 & 4990 & 198.6138 & 0.00079 & 0.040322 & 2096.55 & 119.59 \\
\hline 3.0678 & 313.552 & 1.587 & 0.0774 & 0.2431 & 592.842 & 4990 & 198.1003 & 0.000792 & 0.039559 & 2084.05 & 119.91 \\
\hline 7.0483 & 313.552 & 1.588 & 0.2633 & 0.442 & 584.171 & 4990 & 198.5818 & 0.00079 & 0.040275 & 2095.8 & 119.61 \\
\hline 3.1566 & 314.068 & 1.587 & 0.0769 & 0.2443 & 595.03 & 4990 & 198.0056 & 0.000792 & 0.03942 & 2081.7 & 119.97 \\
\hline 7.5511 & 314.068 & 1.58 & 0.2 & 0.4 & 586.052 & 4990 & 198.508 & 0.000791 & 0.040163 & 2093.95 & 119.65 \\
\hline 3.0639 & 314.888 & 1.587 & 0.0761 & 0.2435 & 597.392 & 4990 & 197.8959 & 0.000792 & 0.039259 & 2079.05 & 120.04 \\
\hline 7.5053 & 314.888 & 1.588 & 0.264 & 0.4429 & 588.781 & 4990 & 198.3804 & 0.000791 & 0.039972 & 2090.8 & 119.74 \\
\hline 3.0593 & 313.419 & 1.587 & 0.0805 & 02494 & 593.337 & 4990 & 198.0774 & 0.000792 & 0.039526 & 20835 & 119.93 \\
\hline 7.6294 & 313.419 & 1.588 & 0.2703 & 0.4 & 584.531 & 4990 & 198.5758 & 0.00079 & 0.040265 & 2095.65 & 119.61 \\
\hline 3.3353 & 313.693 & 1.587 & 0.0801 & 0.2484 & 593.485 & 4990 & 198.0744 & 0.000792 & 0.039521 & 2083.4 & 119.93 \\
\hline 7.8798 & 313.693 & 1.588 & 0.2691 & 0.4527 & 584.675 & 4990 & 198.5708 & 0.00079 & 0.040258 & 2095.45 & 119.62 \\
\hline 3.266 & 314.531 & 1.587 & 0.0801 & 0.248 & 593.645 & 4990 & 198.0665 & 0.000792 & 0.039509 & 2083.2 & 119.93 \\
\hline 7.8661 & 314.531 & 1.588 & 0.2687 & 0.4516 & 584.773 & 4990 & 198.5658 & 0.00079 & 0.04025 & 2095.4 & 119.62 \\
\hline 3.2164 & 315.298 & 1.587 & 0.0795 & 0.2476 & 594.914 & 4990 & 198.0126 & 0.000792 & 0.039428 & 2081.9 & 119.97 \\
\hline 7.9086 & 315.298 & 1.588 & 0.2685 & 0.4516 & 585.83 & 4990 & 198.525 & 0.000791 & 0.04019 & 2094.35 & 119.64 \\
\hline
\end{tabular}




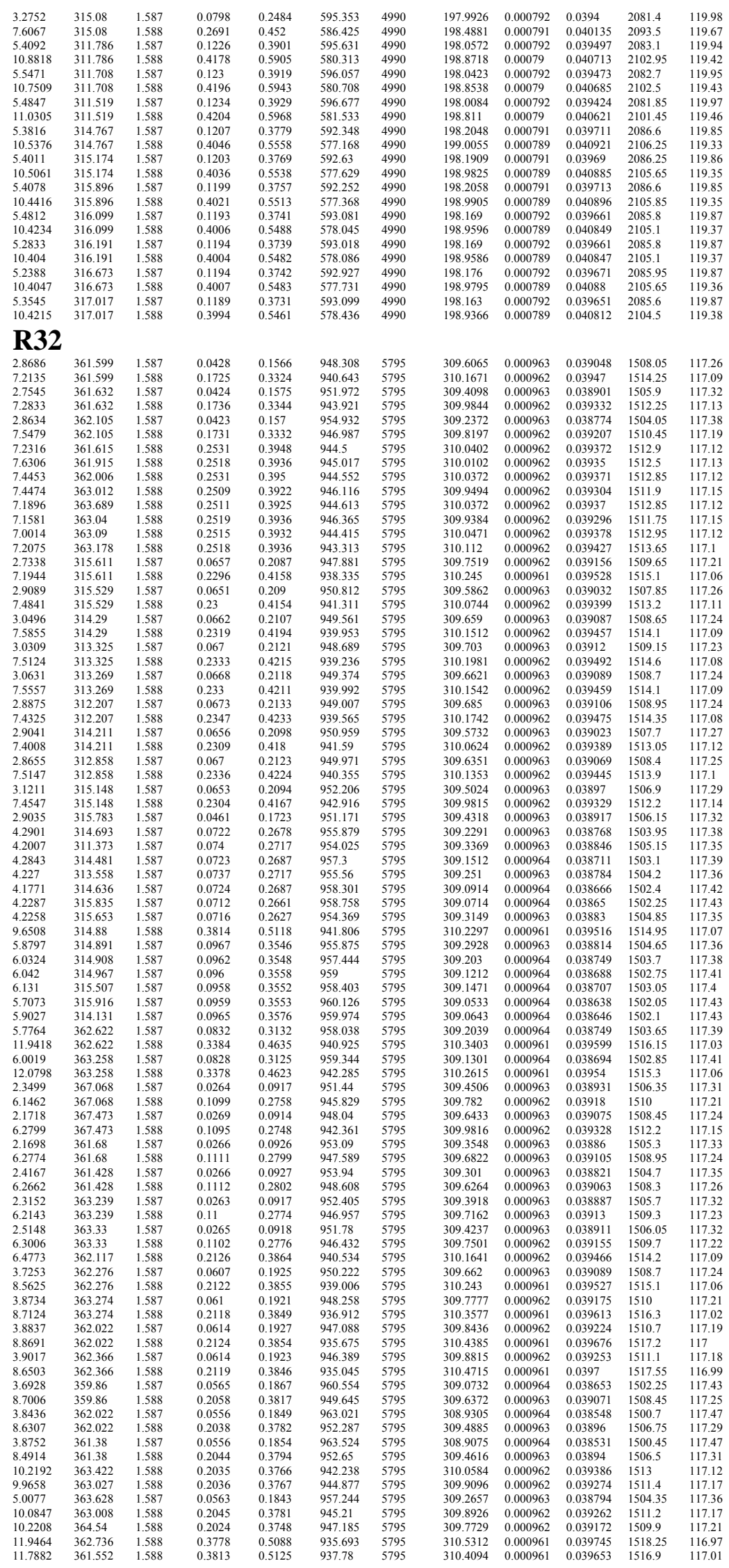




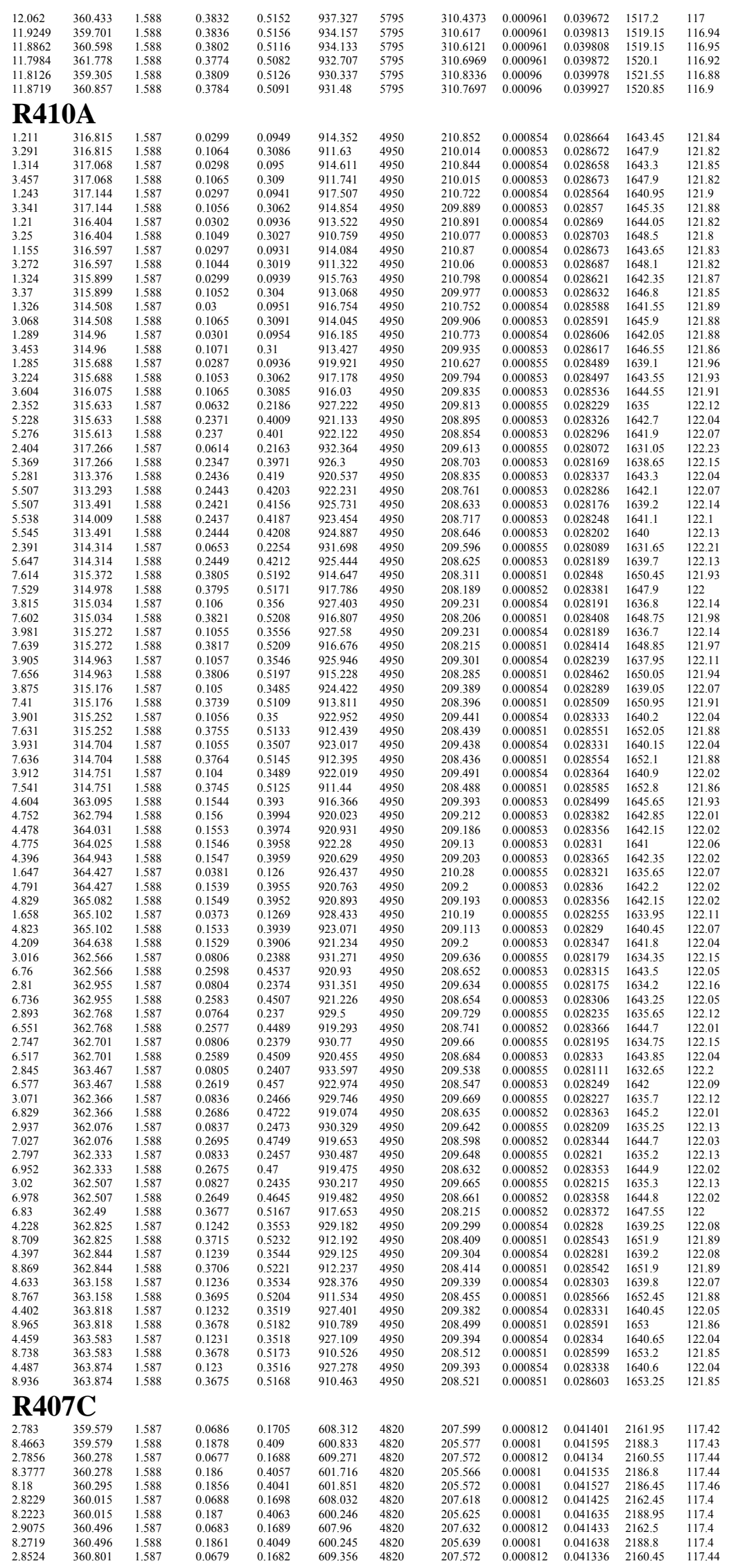




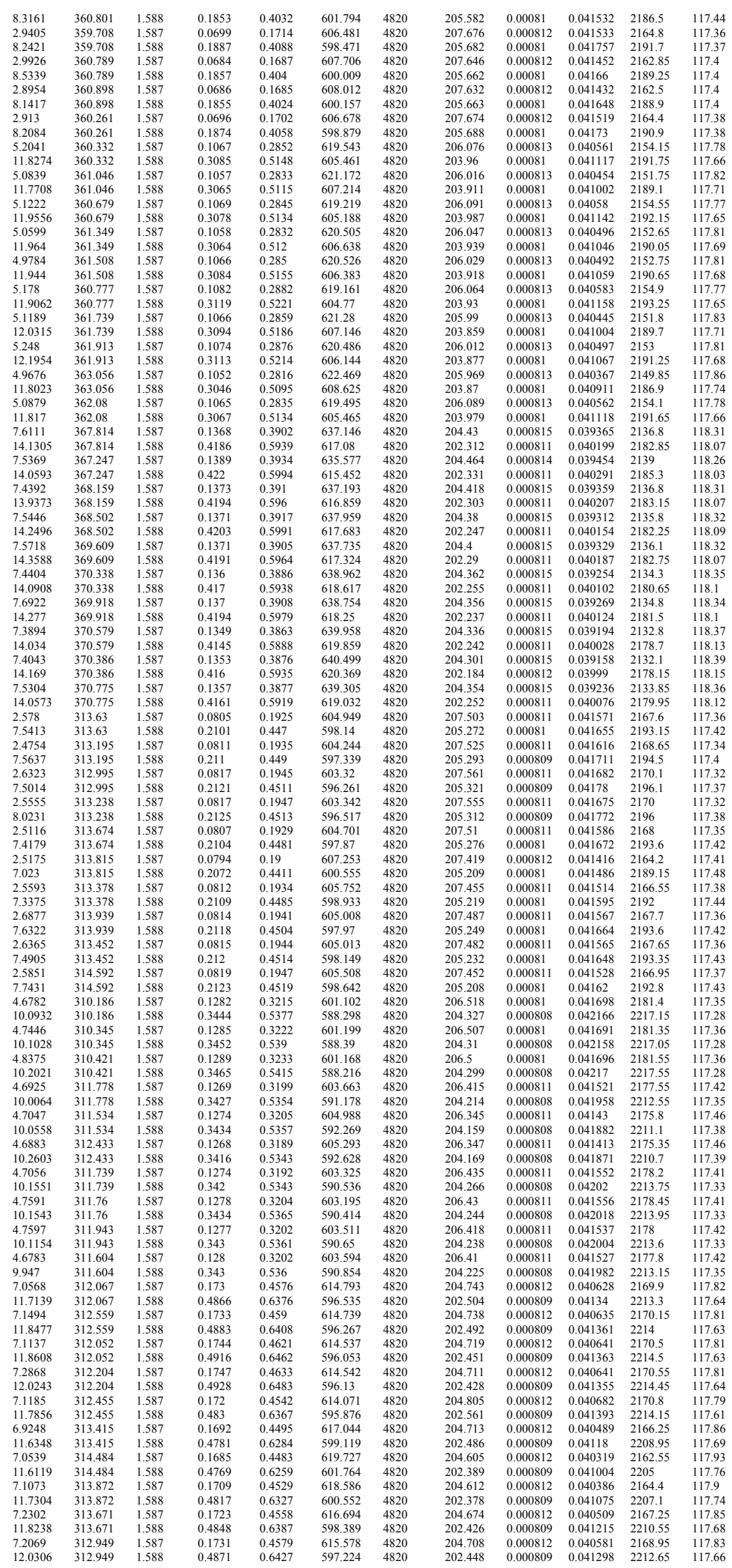




\section{APPENDIX C}

Condensation pressure drop of Kansas State University (file: kan_all.in)

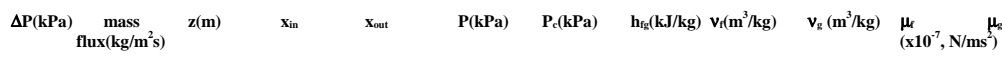

\section{Smooth $3 / 8$ inch}

R22

$\begin{array}{llll}121 & 3.81 & 0.89 & 0.08 \\ 122 & 3.81 & 0.89 & 0.05 \\ 120 & 3.81 & 0.91 & 0.28 \\ 131 & 3.81 & 0.75 & 0.22 \\ 120 & 3.81 & 0.67 & 0.08 \\ 127 & 3.81 & 0.86 & 0.09 \\ 123 & 3.81 & 0.92 & 0.13 \\ 119 & 3.81 & 0.83 & 0.27 \\ 120 & 3.81 & 0.68 & 0.13 \\ 254 & 3.81 & 0.87 & 0.06 \\ 255 & 3.81 & 0.88 & 0.08 \\ 251 & 3.81 & 0.85 & 0.4 \\ 259 & 3.81 & 0.73 & 0.25 \\ 242 & 3.81 & 0.55 & 0.07 \\ 250 & 3.81 & 0.89 & 0.1 \\ 247 & 3.81 & 0.88 & 0.4 \\ 249 & 3.81 & 0.76 & 0.26 \\ 249 & 3.81 & 0.6 & 0.13 \\ 415 & 3.81 & 0.88 & 0.08 \\ 416 & 3.81 & 0.87 & 0.08 \\ 397 & 3.81 & 0.88 & 0.35 \\ 400 & 3.81 & 0.74 & 0.26 \\ 400 & 3.81 & 0.57 & 0.08 \\ 400 & 3.81 & 0.91 & 0.13 \\ 385 & 3.81 & 0.91 & 0.37 \\ 391 & 3.81 & 0.73 & 0.1 \\ 395 & 3.81 & 0.51 & 0.08 \\ 615 & 3.81 & 0.89 & 0.13 \\ 617 & 3.81 & 0.88 & 0.13 \\ 593 & 3.81 & 0.87 & 0.39 \\ 590 & 3.81 & 0.72 & 0.23 \\ 595 & 3.81 & 0.48 & 0.08 \\ & & & \end{array}$

$\begin{array}{lllllll}1542 & 4990 & 163.7 & 0.00089 & 0.01478 & 1459 & 138 \\ 1544 & 4990 & 163.7 & 0.00089 & 0.01474 & 1458 & 138 \\ 1535 & 4990 & 164 & 0.00089 & 0.01483 & 1462 & 137.9 \\ 1550 & 4990 & 163.4 & 0.00089 & 0.01468 & 1456 & 138.1 \\ 1513 & 4990 & 164.7 & 0.00088 & 0.01506 & 1472 & 137.5 \\ 1927 & 4990 & 151.8 & 0.00092 & 0.0115 & 1301 & 144.3 \\ 1990 & 4990 & 150 & 0.00093 & 0.01108 & 1277 & 145.3 \\ 1955 & 4990 & 151.1 & 0.00093 & 0.01131 & 1290 & 144.8 \\ 1942 & 4990 & 151.4 & 0.00093 & 0.0114 & 1295 & 144.6 \\ 1550 & 4990 & 164.1 & 0.00089 & 0.01489 & 1464 & 137.8 \\ 1524 & 4990 & 164.3 & 0.00089 & 0.01496 & 1467 & 137.7 \\ 1515 & 4990 & 164.6 & 0.00088 & 0.01505 & 1471 & 137.5 \\ 1526 & 4990 & 164.2 & 0.00089 & 0.01493 & 1466 & 137.7 \\ 1514 & 4990 & 164.6 & 0.00088 & 0.01506 & 1472 & 137.5 \\ 1955 & 4990 & 151.1 & 0.00093 & 0.01131 & 1290 & 144.8 \\ 1518 & 4990 & 152.1 & 0.00092 & 0.01157 & 1304 & 144.2 \\ 1922 & 4990 & 152 & 0.00092 & 0.01154 & 1303 & 144.2 \\ 1939 & 4990 & 151.5 & 0.00093 & 0.01142 & 1296 & 144.5 \\ 1547 & 4990 & 163.6 & 0.00089 & 0.01471 & 1457 & 138.1 \\ 1551 & 4990 & 163.8 & 0.00089 & 0.01478 & 1460 & 138 \\ 1545 & 4990 & 163.6 & 0.00089 & 0.01473 & 1458 & 138 \\ 1526 & 4990 & 164.2 & 0.00089 & 0.01493 & 1466 & 137.7 \\ 1536 & 4990 & 164 & 0.00089 & 0.01483 & 1462 & 137.9 \\ 1950 & 4990 & 151.1 & 0.00093 & 0.01135 & 1292 & 144.7 \\ 1954 & 4990 & 151 & 0.00093 & 0.01132 & 1291 & 144.7 \\ 1961 & 4990 & 150.9 & 0.00093 & 0.0127 & 1288 & 144.9 \\ 1930 & 4990 & 151.7 & 0.00092 & 0.01148 & 1300 & 144.4 \\ 1514 & 4990 & 164.6 & 0.00088 & 0.01506 & 1472 & 137.5 \\ 1511 & 4990 & 164.7 & 0.00088 & 0.0151 & 1473 & 137.3 \\ 1493 & 4990 & 165.3 & 0.00088 & 0.01529 & 1481 & 137.2 \\ 1559 & 4990 & 163.2 & 0.00089 & 0.01459 & 1451 & 138.3 \\ 1543 & 4990 & 163.7 & 0.00089 & 0.01475 & 1459 & 138\end{array}$

\section{R134a}

$\begin{array}{llll}3.81 & 0.85 & 0.04 & 993 \\ 3.81 & 0.84 & 0.03 & 972 \\ 3.81 & 0.88 & 0.42 & 1029 \\ 3.81 & 0.76 & 0.31 & 1030 \\ 3.81 & 0.57 & 0.14 & 1016 \\ 3.81 & 0.55 & 0.09 & 1307 \\ 3.81 & 0.8 & 0.29 & 1318 \\ 3.81 & 0.9 & 0.39 & 1296 \\ 3.81 & 0.85 & 0.12 & 1290 \\ 3.81 & 0.86 & 0.03 & 1008 \\ 3.81 & 0.86 & 0.02 & 1003 \\ 3.81 & 0.88 & 0.33 & 1023 \\ 3.81 & 0.77 & 0.22 & 1024 \\ 3.81 & 0.46 & 0.06 & 1013 \\ 3.81 & 0.88 & 0.06 & 1316 \\ 3.81 & 0.87 & 0.36 & 1326 \\ 3.81 & 0.75 & 0.19 & 1319 \\ 3.81 & 0.49 & 0.06 & 1300 \\ 3.81 & 0.91 & 0.13 & 1006 \\ 3.81 & 0.91 & 0.13 & 1004 \\ 3.81 & 0.89 & 0.39 & 1015 \\ 3.81 & 0.73 & 0.25 & 1015 \\ 3.81 & 0.52 & 0.12 & 1015 \\ 3.81 & 0.86 & 0.12 & 1301 \\ 3.81 & 0.87 & 0.35 & 1316 \\ 3.81 & 0.77 & 0.26 & 1304 \\ 3.81 & 0.52 & 0.06 & 1302\end{array}$

$\begin{array}{llllll}4067 & 164.1 & 0.00087 & 0.02049 & 1725 & 128 \\ 4067 & 165 & 0.00087 & 0.02096 & 1744 & 127.6 \\ 4067 & 162.7 & 0.00087 & 0.01974 & 1694 & 128.7 \\ 4067 & 162.7 & 0.00087 & 0.01972 & 1693 & 128.8 \\ 4067 & 163.2 & 0.00087 & 0.02 & 1705 & 128.5 \\ 4067 & 152.4 & 0.00091 & 0.01525 & 1489 & 134.1 \\ 4067 & 152.1 & 0.00091 & 0.01511 & 1482 & 134.3 \\ 4067 & 152.8 & 0.0009 & 0.01539 & 1496 & 133.8 \\ 4067 & 153 & 0.0009 & 0.01574 & 1500 & 133.7 \\ 4067 & 163.6 & 0.00087 & 0.02017 & 1712 & 128.3 \\ 4067 & 163.8 & 0.00087 & 0.02028 & 1717 & 128.2 \\ 4067 & 163 & 0.00087 & 0.01986 & 1699 & 128.6 \\ 4067 & 162.9 & 0.00087 & 0.01984 & 1699 & 128.6 \\ 4067 & 163.4 & 0.00087 & 0.02007 & 1708 & 128.4 \\ 4067 & 152.1 & 0.00091 & 0.01514 & 1483 & 134.2 \\ 4067 & 151.7 & 0.00091 & 0.01501 & 1477 & 134.4 \\ 4067 & 152 & 0.00091 & 0.0151 & 1481 & 134.3 \\ 4067 & 152.7 & 0.00091 & 0.01534 & 1494 & 133.9 \\ 4067 & 163.6 & 0.00087 & 0.02021 & 1714 & 128.3 \\ 4067 & 163.7 & 0.00087 & 0.02026 & 1716 & 128.2 \\ 4067 & 163.3 & 0.00087 & 0.02002 & 1706 & 128.5 \\ 4067 & 163.3 & 0.00087 & 0.02002 & 1706 & 128.5 \\ 4067 & 163.3 & 0.00087 & 0.02002 & 1706 & 128.5 \\ 4067 & 152.7 & 0.00091 & 0.01533 & 1493 & 133.9 \\ 4067 & 152.1 & 0.00091 & 0.01514 & 1483 & 134.2 \\ 4067 & 152.5 & 0.00091 & 0.01529 & 1491 & 134 \\ 4067 & 152.6 & 0.00091 & 0.01531 & 1492 & 134\end{array}$

R410A

$\begin{array}{ll}0.61 & 126 \\ 0.75 & 128 \\ 0.45 & 127 \\ 0.65 & 126 \\ 0.61 & 132 \\ 0.48 & 131 \\ 0.8 & 129 \\ 0.54 & 130 \\ 0.52 & 126 \\ 1.85 & 266 \\ 1.36 & 267 \\ 2.12 & 249 \\ 1.73 & 243 \\ 1.15 & 242 \\ 1.37 & 260 \\ 1.49 & 253 \\ 1.9 & 242 \\ 1.2 & 256 \\ 4.15 & 405 \\ 4.18 & 405 \\ 5.23 & 400 \\ 4.57 & 403 \\ 2.97 & 400 \\ 3 & 404 \\ 4.2 & 398 \\ 3.1 & 398 \\ 2.31 & 395 \\ 8.1 & 605\end{array}$

$\begin{array}{lll}3.81 & 0.85 & 0.18 \\ 3.81 & 0.85 & 0.14 \\ 3.81 & 0.85 & 0.44 \\ 3.81 & 0.75 & 0.18 \\ 3.81 & 0.61 & 0.09 \\ 3.81 & 0.87 & 0.1 \\ 3.81 & 0.87 & 0.23 \\ 3.81 & 0.71 & 0.18 \\ 3.81 & 0.49 & 0.08 \\ 3.81 & 0.85 & 0.11 \\ 3.81 & 0.85 & 0.11 \\ 3.81 & 0.85 & 0.38 \\ 3.81 & 0.78 & 0.27 \\ 3.81 & 0.51 & 0.1 \\ 3.81 & 0.86 & 0.09 \\ 3.81 & 0.84 & 0.34 \\ 3.81 & 0.72 & 0.23 \\ 3.81 & 0.5 & 0.09 \\ 3.81 & 0.86 & 0.14 \\ 3.81 & 0.86 & 0.14 \\ 3.81 & 0.86 & 0.41 \\ 3.81 & 0.76 & 0.28 \\ 3.81 & 0.5 & 0.09 \\ 3.81 & 0.85 & 0.1 \\ 3.81 & 0.84 & 0.37 \\ 3.81 & 0.75 & 0.25 \\ 3.81 & 0.48 & 0.05 \\ 3.81 & 0.85 & 0.13\end{array}$

$\begin{array}{ll}2422 & 4950 \\ 2426 & 4950 \\ 2453 & 4950 \\ 2469 & 4950 \\ 2407 & 4950 \\ 2840 & 4950 \\ 2866 & 4950 \\ 2913 & 4950 \\ 2901 & 4950 \\ 2401 & 4950 \\ 2402 & 4950 \\ 2436 & 4950 \\ 2442 & 4950 \\ 2420 & 4950 \\ 2886 & 4950 \\ 2859 & 4950 \\ 2877 & 4950 \\ 2882 & 4950 \\ 2413 & 4950 \\ 2408 & 4950 \\ 2412 & 4950 \\ 2470 & 4950 \\ 2406 & 4950 \\ 2822 & 4950 \\ 2812 & 4950 \\ 2831 & 4950 \\ 2805 & 4950 \\ 2455 & 4950 \\ \end{array}$

$\begin{array}{lllll}166.6 & 0.00101 & 0.01005 & 1000 & 142.6 \\ 166.5 & 0.00102 & 0.01003 & 999 & 142.7 \\ 165.5 & 0.00102 & 0.00989 & 992 & 143.1 \\ 165 & 0.00102 & 0.00981 & 988 & 143.3 \\ 167.1 & 0.00101 & 0.01012 & 1003 & 142.4 \\ 152.3 & 0.00106 & 0.00821 & 909 & 148.9 \\ 151.4 & 0.00106 & 0.00811 & 904 & 149.3 \\ 149.8 & 0.00107 & 0.00794 & 895 & 150 \\ 150.2 & 0.00107 & 0.00798 & 897 & 149.8 \\ 167.3 & 0.00101 & 0.01015 & 1005 & 142.3 \\ 167.2 & 0.00101 & 0.01015 & 1004 & 142.3 \\ 166.1 & 0.00102 & 0.00998 & 996 & 142.8 \\ 165.9 & 0.00102 & 0.00995 & 995 & 142.9 \\ 166.7 & 0.00101 & 0.01006 & 1000 & 142.6 \\ 150.7 & 0.00107 & 0.00804 & 900 & 149.6 \\ 151.7 & 0.00106 & 0.00814 & 905 & 149.2 \\ 151 & 0.00106 & 0.00807 & 902 & 149.5 \\ 150.9 & 0.00106 & 0.00805 & 901 & 149.5 \\ 166.9 & 0.00101 & 0.01009 & 1002 & 142.5 \\ 167 & 0.00101 & 0.01012 & 1003 & 142.4 \\ 166.9 & 0.00101 & 0.0101 & 1002 & 142.5 \\ 165 & 0.00102 & 0.00981 & 988 & 143.3 \\ 167.1 & 0.00101 & 0.01013 & 1003 & 142.4 \\ 152.9 & 0.00106 & 0.00828 & 912 & 148.6 \\ 153 & 0.00106 & 0.00828 & 913 & 148.6 \\ 153.2 & 0.00106 & 0.00825 & 911 & 148.7 \\ 153.5 & 0.00106 & 0.00835 & 916 & 148.3 \\ 165.5 & 0.00102 & 0.00988 & 992 & 143.1\end{array}$




\begin{tabular}{|c|c|c|c|c|c|c|c|c|c|c|c|}
\hline 8.02 & 606 & 3.81 & 0.85 & 0.12 & 2465 & 4950 & 165.1 & 0.00102 & 0.00983 & 989 & 143.2 \\
\hline 13.6 & 599 & 3.81 & 0.85 & 0.48 & 2416 & 4950 & 166.8 & 0.00101 & 0.01008 & 1001 & 142.5 \\
\hline 9.7 & 599 & 3.81 & 0.75 & 0.25 & 2418 & 4950 & 166.7 & 0.00101 & 0.01007 & 1001 & 142.5 \\
\hline 5.76 & 600 & 3.81 & 0.48 & 0.12 & 2431 & 4950 & 166.2 & 0.00102 & 0.01 & 997 & 142.7 \\
\hline \multicolumn{12}{|c|}{ R502 } \\
\hline 1.86 & 251 & 3.81 & 0.86 & 0.11 & 1653 & 4080 & 108.7 & 0.00089 & 0.00992 & 1314 & 141.9 \\
\hline 1.55 & 251 & 3.81 & 0.86 & 0.12 & 1653 & 4080 & 108.7 & 0.00089 & 0.00992 & 1314 & 141.9 \\
\hline 1.87 & 249 & 3.81 & 0.86 & 0.32 & 1676 & 4080 & 108.2 & 0.00089 & 0.00977 & 1300 & 142.3 \\
\hline 1.76 & 249 & 3.81 & 0.75 & 0.24 & 1671 & 4080 & 108.2 & 0.00089 & 0.0098 & 1303 & 142.2 \\
\hline 1.3 & 255 & 3.81 & 0.48 & 0.06 & 1664 & 4080 & 108.5 & 0.00089 & 0.00985 & 1307 & 142.1 \\
\hline 1.27 & 252 & 3.81 & 0.89 & 0.04 & 2060 & 4080 & 97.1 & 0.00094 & 0.00762 & 1082 & 150.2 \\
\hline 2.16 & 253 & 3.81 & 0.87 & 0.37 & 2074 & 4080 & 96.7 & 0.00095 & 0.00755 & 1075 & 150.5 \\
\hline 1.31 & 255 & 3.81 & 0.74 & 0.22 & 2059 & 4080 & 97.2 & 0.00094 & 0.00762 & 1083 & 150.2 \\
\hline 1.31 & 250 & 3.81 & 0.54 & 0.11 & 2061 & 4080 & 97.1 & 0.00094 & 0.00761 & 1082 & 150.2 \\
\hline 4.51 & 404 & 3.81 & 0.85 & 0.15 & 1672 & 4080 & 108.2 & 0.00089 & 0.00979 & 1302 & 142.2 \\
\hline 5.17 & 403 & 3.81 & 0.85 & 0.17 & 1689 & 4080 & 107.7 & 0.00089 & 0.00968 & 1291 & 142.6 \\
\hline 5.66 & 405 & 3.81 & 0.89 & 0.37 & 1701 & 4080 & 107.4 & 0.0009 & 0.0096 & 1284 & 142.8 \\
\hline 4.76 & 396 & 3.81 & 0.72 & 0.22 & 1680 & 4080 & 108 & 0.00089 & 0.00974 & 1297 & 142.4 \\
\hline 3.04 & 401 & 3.81 & 0.52 & 0.07 & 1646 & 4080 & 109 & 0.00089 & 0.00997 & 1319 & 141.7 \\
\hline 3.03 & 403 & 3.81 & 0.87 & 0.08 & 2087 & 4080 & 96.3 & 0.00095 & 0.00749 & 1069 & 150.8 \\
\hline 5.17 & 400 & 3.81 & 0.87 & 0.46 & 2078 & 4080 & 96.6 & 0.00095 & 0.00753 & 1073 & 150.6 \\
\hline 3.76 & 398 & 3.81 & 0.78 & 0.22 & 2084 & 4080 & 96.4 & 0.00095 & 0.00751 & 1070 & 150.7 \\
\hline 2.14 & 402 & 3.81 & 0.48 & 0.04 & 2038 & 4080 & 97.8 & 0.00094 & 0.00772 & 1093 & 149.7 \\
\hline 8.55 & 602 & 3.81 & 0.88 & 0.06 & 1639 & 4080 & 109.2 & 0.00089 & 0.01002 & 1324 & 141.6 \\
\hline 8.25 & 603 & 3.81 & 0.89 & 0.06 & 1638 & 4080 & 109.2 & 0.00089 & 0.01003 & 1324 & 141.6 \\
\hline 13.48 & 600 & 3.81 & 0.86 & 0.37 & 1657 & 4080 & 108.7 & 0.00089 & 0.0099 & 1312 & 141.9 \\
\hline 11.18 & 605 & 3.81 & 0.77 & 0.28 & 1674 & 4080 & 108.1 & 0.00089 & 0.00978 & 1301 & 142.3 \\
\hline 6.24 & 606 & 3.81 & 0.46 & 0.11 & 1650 & 4080 & 108.8 & 0.00089 & 0.00995 & 1316 & 141.8 \\
\hline 5.79 & 605 & 3.81 & 0.91 & 0.07 & 2096 & 4080 & 96 & 0.00095 & 0.00745 & 1064 & 151 \\
\hline 9.07 & 599 & 3.81 & 0.86 & 0.39 & 2077 & 4080 & 96.6 & 0.00095 & 0.00754 & 1074 & 150.6 \\
\hline 6.58 & 603 & 3.81 & 0.73 & 0.16 & 2084 & 4080 & 96.4 & 0.00095 & 0.00751 & 1070 & 150.7 \\
\hline 5.02 & 596 & 3.81 & 0.57 & 0.07 & 2070 & 4080 & 96.8 & 0.00094 & 0.00757 & 1077 & 150.4 \\
\hline \multicolumn{12}{|c|}{ R507a } \\
\hline 0.89 & 131 & 3.81 & 0.823 & 0.075 & 1899 & 3790 & 115.2 & 0.00104 & 0.00915 & 1092 & 140.1 \\
\hline 0.66 & 132 & 3.81 & 0.814 & 0.091 & 1888 & 3790 & 115.6 & 0.00104 & 0.00922 & 1096 & 139.9 \\
\hline 0.79 & 133 & 3.81 & 0.905 & 0.09 & 2456 & 3790 & 95.7 & 0.00113 & 0.00646 & 916 & 152.4 \\
\hline 1.42 & 248 & 3.81 & 0.878 & 0.045 & 1889 & 3790 & 115.6 & 0.00104 & 0.00921 & 1095 & 139.9 \\
\hline 1.56 & 247 & 3.81 & 0.882 & 0.065 & 1878 & 3790 & 116 & 0.00104 & 0.00928 & 1098 & 139.7 \\
\hline 2.46 & 246 & 3.81 & 0.88 & 0.409 & 1858 & 3790 & 116.6 & 0.00103 & 0.00941 & 1104 & 139.3 \\
\hline 2.3 & 245 & 3.81 & 0.788 & 0.194 & 1917 & 3790 & 114.6 & 0.00104 & 0.00904 & 1087 & 140.4 \\
\hline 1.13 & 248 & 3.81 & 0.571 & 0.03 & 1910 & 3790 & 114.8 & 0.00104 & 0.00908 & 1089 & 140.3 \\
\hline 1.34 & 256 & 3.81 & 0.879 & 0.087 & 2427 & 3790 & 96.8 & 0.00113 & 0.00658 & 927 & 151.7 \\
\hline 1.81 & 255 & 3.81 & 0.845 & 0.328 & 2379 & 3790 & 98.5 & 0.00112 & 0.00677 & 945 & 150.5 \\
\hline 1.67 & 255 & 3.81 & 0.718 & 0.252 & 2394 & 3790 & 98 & 0.00112 & 0.00671 & 940 & 150.8 \\
\hline 1.03 & 253 & 3.81 & 0.487 & 0.031 & 2441 & 3790 & 96.3 & 0.00113 & 0.00652 & 921 & 152 \\
\hline 3.93 & 400 & 3.81 & 0.874 & 0.106 & 1880 & 3790 & 115.9 & 0.00104 & 0.00927 & 1098 & 139.7 \\
\hline 3.64 & 399 & 3.81 & 0.866 & 0.125 & 1863 & 3790 & 116.4 & 0.00103 & 0.00937 & 1103 & 139.4 \\
\hline 5.51 & 395 & 3.81 & 0.843 & 0.389 & 1874 & 3790 & 116 & 0.00104 & 0.00931 & 1100 & 139.6 \\
\hline 4.36 & 392 & 3.81 & 0.757 & 0.318 & 1866 & 3790 & 116.3 & 0.00103 & 0.00936 & 1102 & 139.4 \\
\hline 2.42 & 398 & 3.81 & 0.483 & 0.088 & 1910 & 3790 & 114.8 & 0.00104 & 0.00908 & 1089 & 140.3 \\
\hline 2.74 & 396 & 3.81 & 0.858 & 0.062 & 2392 & 3790 & 98.1 & 0.00112 & 0.00672 & 940 & 150.8 \\
\hline 3.43 & 397 & 3.81 & 0.879 & 0.185 & 2369 & 3790 & 99 & 0.00112 & 0.00681 & 949 & 150.2 \\
\hline 2.81 & 391 & 3.81 & 0.765 & 0.139 & 2378 & 3790 & 98.6 & 0.00112 & 0.00677 & 946 & 150.5 \\
\hline 1.99 & 400 & 3.81 & 0.528 & 0.016 & 2387 & 3790 & 98.3 & 0.00112 & 0.00674 & 942 & 150.7 \\
\hline 8.24 & 608 & 3.81 & 0.844 & 0.16 & 1904 & 3790 & 115 & 0.00104 & 0.00912 & 1091 & 140.2 \\
\hline 8.32 & 606 & 3.81 & 0.849 & 0.157 & 1903 & 3790 & 115 & 0.00104 & 0.00913 & 1091 & 140.2 \\
\hline $\begin{array}{l}0.82 \\
10.84\end{array}$ & 598 & 3.81 & 0.839 & 0.368 & 1849 & 3790 & 116.9 & 0.00103 & 0.00946 & 1107 & 139.1 \\
\hline 7.94 & 593 & 3.81 & 0.703 & 0.167 & 1917 & 3790 & 114.6 & 0.00104 & 0.00904 & 1087 & 140.4 \\
\hline 4.76 & 604 & 3.81 & 0.423 & 0.046 & 1926 & 3790 & 114.3 & 0.00104 & 0.00899 & 1085 & 140.6 \\
\hline 5.96 & 601 & 3.81 & 0.867 & 0.096 & 2376 & 3790 & 98.6 & 0.00112 & 0.00678 & 946 & 150.4 \\
\hline 7.87 & 595 & 3.81 & 0.894 & 0.394 & 2369 & 3790 & 99 & 0.00112 & 0.00681 & 949 & 150.2 \\
\hline 6.7 & 593 & 3.81 & 0.761 & 0.266 & 2381 & 3790 & 98.5 & 0.00112 & 0.00676 & 944 & 150.5 \\
\hline 4.62 & 589 & 3.81 & 0.522 & 0.087 & 2357 & 3790 & 99.4 & 0.00111 & 0.00686 & 954 & 150 \\
\hline
\end{tabular}

\section{Micro-fin 3/8 inch}

\section{R22}

\begin{tabular}{|c|c|c|c|c|c|c|c|c|c|c|c|}
\hline 1.06 & 130 & 3.78 & 0.89 & 0.12 & 1502 & 4990 & 165.1 & 0.00088 & 0.01519 & 1477 & 137.3 \\
\hline 1.34 & 130 & 3.78 & 0.89 & 0.15 & 1503 & 4990 & 165 & 0.00088 & 0.01518 & 1477 & 137.3 \\
\hline 1.42 & 129 & 3.78 & 0.88 & 0.38 & 1519 & 4990 & 164.5 & 0.00089 & 0.01501 & 1469 & 137.6 \\
\hline 1.27 & 128 & 3.78 & 0.85 & 0.26 & 1528 & 4990 & 164.2 & 0.00089 & 0.01491 & 1465 & 137.7 \\
\hline 1.19 & 128 & 3.78 & 0.61 & 0.08 & 1510 & 4990 & 164.8 & 0.00088 & 0.01511 & 1474 & 137.4 \\
\hline 0.9 & 127 & 3.78 & 0.87 & 0.06 & 1928 & 4990 & 151.8 & 0.00092 & 0.0115 & 1300 & 144.3 \\
\hline 0.9 & 125 & 3.78 & 0.87 & 0.4 & 1939 & 4990 & 151.5 & 0.00093 & 0.01142 & 1296 & 144.5 \\
\hline 1.12 & 121 & 3.78 & 0.85 & 0.26 & 1910 & 4990 & 152.4 & 0.00092 & 0.01162 & 1307 & 144 \\
\hline 0.99 & 130 & 3.78 & 0.48 & 0.12 & 1978 & 4990 & 150.3 & 0.00093 & 0.01116 & 1282 & 145.1 \\
\hline 5.05 & 262 & 3.78 & 0.88 & 0.08 & 1523 & 4990 & 164.3 & 0.00089 & 0.01497 & 1468 & 137.7 \\
\hline 4.88 & 263 & 3.78 & 0.88 & 0.07 & 1514 & 4990 & 164.6 & 0.00088 & 0.01506 & 1472 & 137.5 \\
\hline 6.04 & 261 & 3.78 & 0.88 & 0.3 & 1527 & 4990 & 164.3 & 0.00089 & 0.01492 & 1466 & 137.7 \\
\hline 4.87 & 239 & 3.78 & 0.74 & 0.25 & 1543 & 4990 & 163.7 & 0.00089 & 0.01475 & 1459 & 138 \\
\hline 3.79 & 245 & 3.78 & 0.57 & 0.14 & 1537 & 4990 & 163.9 & 0.00089 & 0.01482 & 1461 & 137.9 \\
\hline 3.22 & 246 & 3.78 & 0.91 & 0.09 & 1911 & 4990 & 152.3 & 0.00092 & 0.01161 & 1307 & 144 \\
\hline 4.24 & 244 & 3.78 & 0.92 & 0.37 & 1944 & 4990 & 151.3 & 0.00093 & 0.01139 & 1294 & 144.6 \\
\hline 4.18 & 243 & 3.78 & 0.74 & 0.3 & 1924 & 4990 & 151.9 & 0.00092 & 0.01152 & 1302 & 144.3 \\
\hline 2.59 & 252 & 3.78 & 0.46 & 0.11 & 1927 & 4990 & 151.8 & 0.00092 & 0.0115 & 1301 & 144.3 \\
\hline 11.33 & 402 & 3.78 & 0.88 & 0.2 & 1600 & 4990 & 161.9 & 0.00089 & 0.01418 & 1433 & 138.9 \\
\hline 11.44 & 403 & 3.78 & 0.89 & 0.2 & 1609 & 4990 & 161.6 & 0.00089 & 0.01409 & 1430 & 139.1 \\
\hline 13.94 & 386 & 3.78 & 0.89 & 0.45 & 1548 & 4990 & 163.5 & 0.00089 & 0.0147 & 1456 & 138.1 \\
\hline 13.41 & 393 & 3.78 & 0.74 & 0.28 & 1521 & 4990 & 164.4 & 0.00089 & 0.01499 & 1469 & 137.6 \\
\hline 7.96 & 397 & 3.78 & 0.5 & 0.07 & 1497 & 4990 & 165.1 & 0.00088 & 0.01525 & 1479 & 137.2 \\
\hline 9.37 & 404 & 3.78 & 0.86 & 0.19 & 1957 & 4990 & 151 & 0.00093 & 0.0113 & 1289 & 144.8 \\
\hline 11.42 & 403 & 3.78 & 0.87 & 0.39 & 1956 & 4990 & 151 & 0.00093 & 0.01131 & 1290 & 144.8 \\
\hline 9.62 & 402 & 3.78 & 0.72 & 0.26 & 1967 & 4990 & 150.7 & 0.00093 & 0.01123 & 1286 & 145 \\
\hline 7.38 & 399 & 3.78 & 0.47 & 0.15 & 1962 & 4990 & 150.8 & 0.00093 & 0.01127 & 1288 & 144.9 \\
\hline 24.17 & 618 & 3.78 & 0.88 & 0.14 & 1523 & 4990 & 164.3 & 0.00089 & 0.01497 & 1468 & 137.7 \\
\hline 23.92 & 618 & 3.78 & 0.88 & 0.14 & 1525 & 4990 & 164.3 & 0.00089 & 0.01494 & 1467 & 137.7 \\
\hline 30.61 & 608 & 3.78 & 0.88 & 0.39 & 1538 & 4990 & 163.9 & 0.00089 & 0.01481 & 1461 & 137.9 \\
\hline 27.22 & 604 & 3.78 & 0.74 & 0.3 & 1529 & 4990 & 164.2 & 0.00089 & 0.0149 & 1465 & 137.8 \\
\hline 18.14 & 596 & 3.78 & 0.51 & 0.14 & 1516 & 4990 & 164.6 & 0.00088 & 0.01504 & 1471 & 137.5 \\
\hline 17.76 & 607 & 3.78 & 0.85 & 0.15 & 1911 & 4990 & 152.3 & 0.00092 & 0.01161 & 1307 & 144 \\
\hline 20.98 & 587 & 3.78 & 0.89 & 0.4 & 1935 & 4990 & 151.6 & 0.00093 & 0.01145 & 1298 & 144.4 \\
\hline 18.78 & 603 & 3.78 & 0.72 & 0.27 & 1933 & 4990 & 151.6 & 0.00092 & 0.01146 & 1299 & 144.4 \\
\hline 12.51 & 595 & 3.78 & 0.46 & 0.11 & 1917 & 4990 & 152.2 & 0.00092 & 0.01157 & 1305 & 144.1 \\
\hline 0.68 & 121 & 3.78 & 0.89 & 0.08 & 1542 & 4990 & 163.7 & 0.00089 & 0.01476 & 1459 & 138 \\
\hline 0.66 & 122 & 3.78 & 0.89 & 0.05 & 1544 & 4990 & 163.7 & 0.00089 & 0.01474 & 1458 & 138 \\
\hline
\end{tabular}




\begin{tabular}{|c|c|c|c|c|c|c|c|c|c|c|c|}
\hline 2.45 & 254 & 3.78 & 0.87 & 0.06 & 1530 & 4990 & 164.1 & 0.00089 & 0.01489 & 1464 & 137.8 \\
\hline 2.52 & 255 & 3.78 & 0.88 & 0.08 & 1524 & 4990 & 164.3 & 0.00089 & 0.01496 & 1467 & 137.7 \\
\hline 7.4 & 415 & 3.78 & 0.88 & 0.08 & 1547 & 4990 & 163.6 & 0.00089 & 0.01471 & 1457 & 138.1 \\
\hline 7.42 & 416 & 3.78 & 0.87 & 0.08 & 1541 & 4990 & 163.8 & 0.00089 & 0.01478 & 1460 & 138 \\
\hline 16.08 & 615 & 3.78 & 0.89 & 0.13 & 1514 & 4990 & 164.6 & 0.00088 & 0.01506 & 1472 & 137.5 \\
\hline 16.78 & 617 & 3.78 & 0.88 & 0.13 & 1511 & 4990 & 164.7 & 0.00088 & 0.0151 & 1473 & 137.5 \\
\hline 1.06 & 130 & 3.78 & 0.89 & 0.12 & 1502 & 4990 & 165.1 & 0.00088 & 0.01519 & 1477 & 137.3 \\
\hline 1.34 & 130 & 3.78 & 0.89 & 0.15 & 1503 & 4990 & 165 & 0.00088 & 0.01518 & 1477 & 137.3 \\
\hline 5.05 & 262 & 3.78 & 0.88 & 0.08 & 1523 & 4990 & 164.3 & 0.00089 & 0.01479 & 1468 & 137.7 \\
\hline 4.88 & 263 & 3.78 & 0.88 & 0.07 & 1514 & 4990 & 164.6 & 0.00088 & 0.01506 & 1472 & 137.5 \\
\hline 11.33 & 402 & 3.78 & 0.88 & 0.2 & 1600 & 4990 & 161.9 & 0.00089 & 0.01418 & 1433 & 138.9 \\
\hline 11.44 & 403 & 3.78 & 0.89 & 0.2 & 1609 & 4990 & 161.6 & 0.00089 & 0.01409 & 1430 & 139.1 \\
\hline 24.17 & 618 & 3.78 & 0.88 & 0.14 & 1523 & 4990 & 164.3 & 0.00089 & 0.01479 & 1468 & 137.7 \\
\hline 23.92 & 618 & 3.78 & 0.88 & 0.14 & 1525 & 4990 & 164.3 & 0.00089 & 0.01494 & 1467 & 137.7 \\
\hline 0.61 & 126 & 3.78 & 0.91 & 0.1 & 1529 & 4990 & 164.2 & 0.00089 & 0.0149 & 1465 & 137.8 \\
\hline 0.78 & 126 & 3.78 & 0.91 & 0.1 & 1534 & 4990 & 164 & 0.00089 & 0.01485 & 1463 & 137.8 \\
\hline 3.2 & 268 & 3.78 & 0.88 & 0.1 & 1529 & 4990 & 164.2 & 0.00089 & 0.0149 & 1465 & 137.8 \\
\hline 3.02 & 268 & 3.78 & 0.89 & 0.1 & 1530 & 4990 & 164.1 & 0.00089 & 0.01489 & 1464 & 137.8 \\
\hline 5.54 & 398 & 3.78 & 0.75 & 0.09 & 1539 & 4990 & 163.9 & 0.00089 & 0.0148 & 1460 & 137.9 \\
\hline 5.16 & 398 & 3.78 & 0.75 & 0.08 & 1536 & 4990 & 164 & 0.00089 & 0.01483 & 1462 & 137.9 \\
\hline 1.44 & 125 & 3.78 & 0.84 & 0.11 & 1534 & 4990 & 164 & 0.00089 & 0.01485 & 1463 & 137.8 \\
\hline 1.44 & 125 & 3.78 & 0.84 & 0.13 & 1538 & 4990 & 163.9 & 0.00089 & 0.01481 & 1461 & 137.9 \\
\hline 5.32 & 252 & 3.78 & 0.89 & 0.15 & 1537 & 4990 & 163.9 & 0.00089 & 0.01482 & 1461 & 137.9 \\
\hline 5.82 & 251 & 3.78 & 0.89 & 0.17 & 1530 & 4990 & 164.1 & 0.00089 & 0.01489 & 1464 & 137.8 \\
\hline 12.52 & 407 & 3.78 & 0.89 & 0.09 & 1554 & 4990 & 163.4 & 0.00089 & 0.01464 & 1454 & 138.2 \\
\hline 12.73 & 406 & 3.78 & 0.9 & 0.09 & 1554 & 4990 & 163.4 & 0.00089 & 0.01464 & 1454 & 138.2 \\
\hline 28.42 & 610 & 3.78 & 0.85 & 0.12 & 1504 & 4990 & 165 & 0.00088 & 0.01517 & 1476 & 137.3 \\
\hline 28.25 & 611 & 3.78 & 0.85 & 0.11 & $\begin{array}{l}1499 \\
\end{array}$ & 4990 & 165.1 & 0.00088 & 0.01523 & 1479 & 137.3 \\
\hline
\end{tabular}

R134a

\begin{tabular}{|c|c|c|c|c|c|c|c|c|c|c|c|}
\hline 6.07 & 251 & 378 & 0.86 & 008 & 1029 & 4067 & 1627 & 000087 & 0.01974 & 1694 & 128.7 \\
\hline 5.93 & 251 & 3.78 & 0.86 & 0.08 & 1030 & 4067 & 162.7 & 0.00087 & 0.01972 & 1693 & 128.8 \\
\hline 7.62 & 249 & 3.78 & 0.85 & 0.3 & 992 & 4067 & 164.2 & 0.00087 & 0.02051 & 1726 & 128 \\
\hline 6.69 & 244 & 3.78 & 0.75 & 0.21 & 1007 & 4067 & 163.6 & 0.00087 & 0.02019 & 1713 & 128.3 \\
\hline 5.19 & 247 & 3.78 & 0.54 & 0.09 & 1006 & 4067 & 163.6 & 0.00087 & 0.02021 & 1714 & 128.3 \\
\hline 4.58 & 252 & 3.78 & 0.88 & 0.05 & 1319 & 4067 & 152 & 0.00091 & 0.0151 & 1481 & 134.3 \\
\hline 5.47 & 249 & 3.78 & 0.9 & 0.3 & 1297 & 4067 & 152.8 & 0.00091 & 0.01538 & 1496 & 133.9 \\
\hline 5.22 & 250 & 3.78 & 0.72 & 0.25 & 1298 & 4067 & 152.7 & 0.00091 & 0.01537 & 1495 & 133.9 \\
\hline 3.71 & 254 & 3.78 & 0.5 & 0.07 & 1294 & 4067 & 152.9 & 0.0009 & 0.01542 & 1498 & 133.8 \\
\hline 14.19 & 398 & 3.78 & 0.88 & 0.11 & 1014 & 4067 & 163.4 & 0.00087 & 0.02004 & 1707 & 128.4 \\
\hline 14.87 & 398 & 3.78 & 0.87 & 0.1 & 1010 & 4067 & 163.5 & 0.00087 & 0.02013 & 1711 & 128.4 \\
\hline 18.06 & 401 & 3.78 & 0.87 & 0.33 & 994 & 4067 & 164.1 & 0.00087 & 0.02047 & 1724 & 128 \\
\hline 16.16 & 397 & 3.78 & 0.74 & 0.21 & 1008 & 4067 & 163.6 & 0.00087 & 0.02017 & 1712 & 128.3 \\
\hline 11.22 & 395 & 3.78 & 0.51 & 0.09 & 1015 & 4067 & 163.3 & 0.00087 & 0.02002 & 1706 & 128.5 \\
\hline 10.94 & 404 & 3.78 & 0.9 & 0.09 & 1328 & 4067 & 151.7 & 0.00091 & 0.01498 & 1475 & 134.4 \\
\hline 13.41 & 395 & 3.78 & 0.89 & 0.31 & 1291 & 4067 & 153 & 0.0009 & 0.01546 & 1499 & 133.8 \\
\hline 12.6 & 399 & 3.78 & 0.74 & 0.24 & 1300 & 4067 & 152.7 & 0.00091 & 0.01534 & 1494 & 133.9 \\
\hline 8.59 & 400 & 3.78 & 0.54 & 0.08 & 1300 & 4067 & 152.7 & 0.00091 & 0.01534 & 1494 & 133.9 \\
\hline 27.6 & 613 & 3.78 & 0.86 & 0.08 & 1011 & 4067 & 163.4 & 0.00087 & 0.02011 & 1710 & 128.4 \\
\hline 36.97 & 589 & 3.78 & 0.88 & 0.35 & 1000 & 4067 & $\begin{array}{l}163.8 \\
163.8\end{array}$ & 0.00087 & 0.02034 & 1719 & $\begin{array}{l}128.4 \\
128.2\end{array}$ \\
\hline 34.06 & 597 & 3.78 & 0.77 & 0.27 & 1001 & 4067 & 163.9 & 0.00087 & 0.02032 & 1718 & 128.2 \\
\hline 19.78 & 603 & 3.78 & 0.46 & 0.06 & 989 & 4067 & 164.5 & 0.00087 & 0.02073 & 1735 & 127.8 \\
\hline 22.06 & 610 & 3.78 & 0.88 & 0.08 & 1298 & 4067 & 152.7 & 0.00091 & 0.01537 & 1495 & 133.9 \\
\hline 27.67 & 585 & 3.78 & 0.88 & 0.34 & 1281 & 4067 & 153.5 & 0.0009 & 0.01559 & 1506 & 133.6 \\
\hline 23.34 & 590 & 3.78 & 0.75 & 0.19 & 1299 & 4067 & 152.7 & 0.00091 & 0.01535 & 1494 & 133.9 \\
\hline $\begin{array}{l}16.92 \\
\end{array}$ & 595 & 3.78 & 0.58 & 0.04 & 1301 & 4067 & 152.7 & 0.00091 & 0.01533 & 1493 & 133.9 \\
\hline 0.92 & 124 & 3.78 & 0.88 & 0.09 & 1009 & 4067 & 163.5 & 0.00087 & 0.02015 & 1711 & 128.3 \\
\hline 0.96 & 124 & 3.78 & 0.88 & 0.09 & 1010 & 4067 & 163.5 & 0.00087 & 0.02013 & 1711 & 128.4 \\
\hline 0.86 & 125 & 3.78 & 0.86 & 0.05 & 1006 & 4067 & 163.6 & 0.00087 & 0.02021 & 1714 & 128.3 \\
\hline 3.35 & 259 & 3.78 & 0.92 & 0.1 & 1010 & 4067 & 163.5 & 0.00087 & 0.02013 & 1711 & 128.4 \\
\hline 3.38 & 259 & 3.78 & 0.91 & 0.1 & 1007 & 4067 & 163.6 & 0.00087 & 0.02019 & 1713 & 128.6 \\
\hline 3.83 & 252 & 3.78 & 0.87 & 0.08 & 1001 & 4067 & 163.9 & 0.00087 & 0.02032 & 1718 & 128.2 \\
\hline 3.15 & 252 & 3.78 & 0.87 & 0.08 & 1002 & 4067 & 163.8 & 0.00087 & 0.02032 & 1717 & 128.2 \\
\hline 5.63 & 348 & 3.78 & 0.89 & 0.1 & 1022 & 4067 & 163 & 0.00087 & 0.01988 & 1700 & 128.6 \\
\hline 5.77 & 351 & 3.78 & 0.87 & 0.1 & 1020 & 4067 & 163.1 & 0.00087 & 0.01992 & 1702 & 128.6 \\
\hline 4.81 & 322 & 3.78 & 0.87 & 0.05 & 1017 & 4067 & 163.2 & 0.00087 & 0.01998 & 1705 & 128.5 \\
\hline 4.61 & 323 & 3.78 & 0.88 & 0.04 & 1020 & 4067 & 163.1 & 0.00087 & 0.01992 & 1702 & 128.6 \\
\hline 1.64 & 128 & 3.78 & 0.91 & 0.04 & 1013 & 4067 & 163.4 & 0.00087 & 0.02007 & 1708 & 128.4 \\
\hline $\begin{array}{l}1.72 \\
\text {. }\end{array}$ & 128 & 3.78 & 0.9 & 0.05 & 1014 & 4067 & 163.4 & 0.00087 & 0.02004 & 1707 & 128.4 \\
\hline 6.26 & 241 & 3.78 & 0.89 & 0.08 & 1017 & 4067 & 163.2 & 0.00087 & 0.01998 & 1705 & 128.5 \\
\hline 6.55 & 241 & 3.78 & 0.89 & 0.07 & 1017 & 4067 & 163.2 & 0.00087 & 0.01998 & 1705 & 128.5 \\
\hline 7.52 & 254 & 3.78 & 0.86 & 0.1 & 1001 & 4067 & 163.9 & 0.00087 & 0.02032 & 1718 & 128.2 \\
\hline 7.55 & 253 & 3.78 & 0.86 & 0.1 & 1001 & 4067 & 163.9 & 0.00087 & 0.02032 & 1718 & 128.2 \\
\hline 15.97 & 388 & 3.78 & 0.87 & 0.08 & 1007 & 4067 & 163.6 & 0.00087 & 0.02019 & 1713 & 128.6 \\
\hline 16.49 & 387 & 3.78 & 0.87 & 0.08 & 1004 & 4067 & 163.7 & 0.00087 & 0.02026 & 1716 & 128.2 \\
\hline 17.59 & 396 & 3.78 & 0.87 & 0.11 & 995 & 4067 & 164.1 & 0.00087 & 0.02045 & 1724 & 128.1 \\
\hline 16.61 & 398 & 3.78 & 0.9 & 0.08 & 1017 & 4067 & 163.2 & 0.00087 & 0.01998 & 1705 & 128.5 \\
\hline 35.35 & 593 & 3.78 & 0.89 & 0.1 & 1010 & 4067 & 163.5 & 0.00087 & 0.02013 & 1711 & 128.4 \\
\hline 35.04 & 592 & 3.78 & 0.89 & 0.1 & 1010 & 4067 & 163.5 & 0.00087 & 0.02013 & 1711 & $\begin{array}{l}128.4 \\
128.4\end{array}$ \\
\hline 52.89 & 791 & 3.78 & 0.88 & 0.09 & 1012 & 4067 & 163.4 & 0.00087 & 0.02009 & 1709 & 128.4 \\
\hline 53.46 & 791 & 3.78 & 0.87 & 0.08 & 1013 & 4067 & 163.4 & 0.00087 & 0.02007 & 1708 & 128.4 \\
\hline \multicolumn{12}{|c|}{ R410A } \\
\hline 0.74 & 122 & 3.78 & 0.81 & 0.07 & 2460 & 4950 & 165.3 & 0.00102 & 0.00986 & 990 & 143.2 \\
\hline 0.75 & 120 & 3.78 & 0.84 & 0.06 & 2465 & 4950 & 165.1 & 0.00102 & 0.00983 & 989 & 143.2 \\
\hline 1.07 & 124 & 3.78 & 0.8 & 0.25 & 2458 & 4950 & 165.4 & 0.00102 & 0.00987 & 991 & 143.1 \\
\hline 0.77 & 124 & 3.78 & 0.76 & 0.18 & 2426 & 4950 & 166.5 & 0.00102 & 0.01003 & 999 & 142.7 \\
\hline 0.77 & 125 & 3.78 & 0.59 & 0.04 & 2410 & 4950 & 167 & 0.00101 & 0.01011 & 1002 & 142.4 \\
\hline 0.69 & 129 & 3.78 & 0.84 & 0.15 & 2871 & 4950 & 151.2 & 0.00106 & 0.0081 & 903 & 149.4 \\
\hline 0.66 & 122 & 3.78 & 0.81 & 0.19 & 2910 & 4950 & 149.9 & 0.00107 & 0.00795 & 895 & 150 \\
\hline 0.94 & 123 & 3.78 & 0.81 & 0.19 & 2859 & 4950 & 151.6 & 0.00106 & 0.00814 & 905 & 149.2 \\
\hline 0.71 & 122 & 3.78 & 0.64 & 0.05 & 2829 & 4950 & 152.7 & 0.00106 & 0.00825 & 911 & 148.7 \\
\hline 2.43 & 253 & 3.78 & 0.85 & 0.1 & 2470 & 4950 & 165 & 0.00102 & 0.00981 & 988 & 143.3 \\
\hline 2.58 & 253 & 3.78 & 0.86 & 0.09 & 2466 & 4950 & 165.1 & 0.00102 & 0.00983 & 989 & 143.2 \\
\hline 3.23 & 256 & 3.78 & 0.83 & 0.24 & 2443 & 4950 & 165.8 & 0.00102 & 0.00994 & 994 & 142.9 \\
\hline 3.12 & 254 & 3.78 & 0.75 & 0.22 & 2399 & 4950 & 167.3 & 0.00101 & 0.01017 & 1005 & 142.3 \\
\hline 2.39 & 256 & 3.78 & 0.54 & 0.09 & 2418 & 4950 & 166.7 & 0.00101 & 0.01007 & 1001 & 142.5 \\
\hline 1.95 & 250 & 3.78 & 0.86 & 0.05 & 2879 & 4950 & 151 & 0.00164 & 0.00807 & 901 & 149.5 \\
\hline 3.5 & 253 & 3.78 & 0.86 & 0.46 & 2872 & 4950 & 151.2 & 0.00106 & 0.00809 & 903 & 149.4 \\
\hline 2.65 & 245 & 3.78 & 0.69 & 0.18 & 2867 & 4950 & 151.3 & 0.00106 & 0.00811 & 904 & 149.3 \\
\hline $\begin{array}{l}2.68 \\
1.68\end{array}$ & 243 & 3.78 & 0.52 & 0.04 & 2805 & 4950 & 153.5 & 0.00106 & 0.00835 & 916 & 148.3 \\
\hline 6.83 & 403 & 3.78 & 0.84 & 0.09 & 2408 & 4950 & 167 & 0.00101 & 0.01012 & 1003 & 142.4 \\
\hline 6.55 & 403 & 3.78 & 0.85 & 0.1 & 2415 & 4950 & 166.8 & 0.00101 & 0.01008 & 1001 & 142.5 \\
\hline 7.14 & 399 & 3.78 & 0.85 & 0.32 & 2471 & 4950 & 164.8 & 0.00102 & 0.0098 & 988 & 143.3 \\
\hline 7.33 & 402 & 3.78 & 0.73 & 0.18 & 2439 & 4950 & 166 & 0.00102 & 0.00996 & 996 & 142.9 \\
\hline 5.32 & 404 & 3.78 & 0.49 & 0.09 & 2429 & 4950 & 166.4 & 0.00102 & 0.01001 & 998 & 142.7 \\
\hline 5.67 & 405 & 3.78 & 0.86 & 0.12 & 2828 & 4950 & 152.7 & 0.00106 & 0.00826 & 911 & 148.7 \\
\hline 6.2 & 404 & 3.78 & 0.86 & 0.32 & 2889 & 4950 & 150.6 & 0.00107 & 0.00803 & 899 & 149.7 \\
\hline 5.95 & 404 & 3.78 & 0.74 & 0.24 & 2897 & 4950 & 150.3 & 0.00107 & 0.008 & 898 & 149.8 \\
\hline
\end{tabular}




$\begin{array}{llllllllllll}4.39 & 402 & 3.78 & 0.48 & 0.08 & 2824 & 4950 & 152.8 & 0.00106 & 0.00827 & 912 & 148.6 \\ 12.52 & 610 & 3.78 & 0.85 & 0.1 & 2454 & 4950 & 165.5 & 0.00102 & 0.00989 & 992 & 143.1 \\ 12.67 & 609 & 3.78 & 0.85 & 0.1 & 2461 & 4950 & 165.3 & 0.00102 & 0.00985 & 990 & 143.2 \\ 16.62 & 603 & 3.78 & 0.86 & 0.37 & 2395 & 4950 & 167.4 & 0.00101 & 0.01019 & 1006 & 142.2 \\ 14.19 & 595 & 3.78 & 0.75 & 0.23 & 2421 & 4950 & 166.6 & 0.00101 & 0.01005 & 1000 & 142.6 \\ 9.83 & 606 & 3.78 & 0.49 & 0.04 & 2403 & 4950 & 167.2 & 0.00101 & 0.01014 & 1004 & 142.3 \\ 0.59 & 125 & 3.78 & 0.86 & 0.06 & 2448 & 4950 & 165.7 & 0.00102 & 0.00992 & 993 & 143 \\ 0.64 & 125 & 3.78 & 0.86 & 0.06 & 2452 & 4950 & 165.5 & 0.00102 & 0.0099 & 992 & 143 \\ 1.73 & 250 & 3.78 & 0.88 & 0.08 & 2470 & 4950 & 165 & 0.00102 & 0.00981 & 988 & 143.3 \\ 1.5 & 250 & 3.78 & 0.88 & 0.08 & 2471 & 4950 & 164.9 & 0.00102 & 0.0098 & 988 & 143.3 \\ 3.44 & 400 & 3.78 & 0.68 & 0.1 & 2391 & 4950 & 167.6 & 0.00101 & 0.01021 & 1007 & 142.2 \\ 3.84 & 400 & 3.78 & 0.68 & 0.11 & 2402 & 4950 & 167.2 & 0.00101 & 0.01015 & 1004 & 142.3 \\ 0.76 & 127 & 3.78 & 0.82 & 0.1 & 2452 & 4950 & 165.5 & 0.00102 & 0.0099 & 992 & 143 \\ 0.98 & 127 & 3.78 & 0.84 & 0.16 & 2455 & 4950 & 165.5 & 0.00102 & 0.00988 & 992 & 143.1 \\ 3.19 & 250 & 3.78 & 0.89 & 0.08 & 2546 & 4950 & 165.7 & 0.00102 & 0.00993 & 994 & 143 \\ 2.89 & 250 & 3.78 & 0.83 & 0.05 & 2464 & 4950 & 165.2 & 0.00102 & 0.00984 & 990 & 143.2 \\ 7.6 & 406 & 3.78 & 0.85 & 0.03 & 2451 & 4950 & 165.6 & 0.00102 & 0.0099 & 993 & 143 \\ 7.11 & 405 & 3.78 & 0.85 & 0.04 & 2464 & 4950 & 165.2 & 0.00102 & 0.00984 & 990 & 143.2 \\ 15.67 & 597 & 3.78 & 0.88 & 0.06 & 2438 & 4950 & 166 & 0.00102 & 0.00997 & 996 & 142.8 \\ 15.42 & 597 & 3.78 & 0.88 & 0.06 & 2429 & 4950 & 166.4 & 0.00102 & 0.01001 & 998 & 142.7\end{array}$

\section{R502}

\begin{tabular}{|c|c|c|c|c|c|c|c|c|c|c|c|}
\hline 2.86 & 248 & 3.78 & 0.86 & 0.04 & 1641 & 4080 & 109.1 & 0.00089 & 0.01001 & 1322 & 141.6 \\
\hline 2.86 & 248 & 3.78 & 0.86 & 0.04 & 1641 & 4080 & 109.1 & 0.00089 & 0.01001 & 1322 & 141.6 \\
\hline 4.36 & 256 & 3.78 & 0.88 & 0.4 & 1693 & 4080 & 107.6 & 0.00089 & 0.00965 & 1289 & 142.7 \\
\hline 4.39 & 245 & 3.78 & 0.77 & 0.32 & 1688 & 4080 & 107.8 & 0.00089 & 0.00969 & 1292 & 142.6 \\
\hline 1.8 & 248 & 3.78 & 0.5 & 0.06 & 1663 & 4080 & 108.5 & 0.00089 & 0.00986 & 1308 & 142.1 \\
\hline 2.26 & 254 & 3.78 & 0.88 & 0.09 & 2117 & 4080 & 95.5 & 0.00095 & 0.00736 & 1054 & 151.4 \\
\hline 4.11 & 257 & 3.78 & 0.85 & 0.6 & 2083 & 4080 & 96.4 & 0.00095 & 0.00751 & 1071 & 150.7 \\
\hline 3.03 & 264 & 3.78 & 0.76 & 0.18 & 2038 & 4080 & 97.8 & 0.00094 & 0.00772 & 1093 & 149.7 \\
\hline 2.07 & 264 & 3.78 & 0.55 & 0.13 & 2091 & 4080 & 96.2 & 0.00095 & 0.00747 & 1067 & 150.9 \\
\hline 7.56 & 402 & 3.78 & 0.83 & 0.12 & 1657 & 4080 & 108.7 & 0.00089 & 0.0099 & 1312 & 141.9 \\
\hline 7.29 & 402 & 3.78 & 0.84 & 0.09 & 1644 & 4080 & 109 & 0.00089 & 0.00999 & 1320 & 141.7 \\
\hline 9.34 & 395 & 3.78 & 0.85 & 0.44 & 1650 & 4080 & 108.8 & 0.00089 & 0.00995 & 1316 & 141.8 \\
\hline 8.38 & 399 & 3.78 & 0.71 & 0.26 & 1664 & 4080 & 108.5 & 0.00089 & 0.00985 & 1307 & 142.1 \\
\hline 5.83 & 401 & 3.78 & 0.48 & 0.11 & 1673 & 4080 & 108.2 & 0.00089 & 0.00979 & 1302 & 142.3 \\
\hline 5.46 & 404 & 3.78 & 0.86 & 0.05 & 2058 & 4080 & 97.2 & 0.00094 & 0.00763 & 1083 & 150.2 \\
\hline 6.38 & 395 & 3.78 & 0.89 & 0.36 & 2077 & 4080 & 96.6 & 0.00095 & 0.00754 & 1074 & 150.6 \\
\hline 5.76 & 397 & 3.78 & 0.67 & 0.27 & 2075 & 4080 & 96.7 & 0.00095 & 0.00755 & 1075 & 150.5 \\
\hline 4.45 & 405 & 3.78 & 0.49 & 0.07 & 2045 & 4080 & 97.6 & 0.00094 & 0.00769 & 1090 & 149.9 \\
\hline 14.05 & 613 & 3.78 & 0.86 & 0.07 & 1671 & 4080 & 108.2 & 0.00089 & 0.0098 & 1303 & 142.2 \\
\hline 14.05 & 613 & 3.78 & 0.86 & 0.07 & 1671 & 4080 & 108.2 & 0.00089 & 0.0098 & 1303 & 142.2 \\
\hline 17.65 & 589 & 3.78 & 0.9 & 0.37 & 1667 & 4080 & 108.4 & 0.00089 & 0.00983 & 1305 & 142.1 \\
\hline 16.67 & 595 & 3.78 & 0.73 & 0.26 & 1640 & 4080 & 109.2 & 0.00089 & 0.01002 & 1323 & 141.6 \\
\hline 10.67 & 603 & 3.78 & 0.49 & 0.08 & 1674 & 4080 & 108.1 & 0.00089 & 0.00978 & 1301 & 142.3 \\
\hline 11 & 606 & 3.78 & 0.86 & 0.05 & 2035 & 4080 & 97.8 & 0.00094 & 0.00773 & 1095 & 149.7 \\
\hline 14.87 & 610 & 3.78 & 0.84 & 0.42 & 2073 & 4080 & 96.7 & 0.00095 & 0.00756 & 1076 & 150.5 \\
\hline 12.26 & 596 & 3.78 & 0.75 & 0.32 & 2115 & 4080 & 95.6 & 0.00095 & 0.00737 & 1055 & 151.4 \\
\hline 8.37 & 604 & 3.78 & 0.46 & 0.1 & 2116 & 4080 & 95.5 & 0.00095 & 0.00736 & 1055 & 151.4 \\
\hline 0.58 & 126 & 3.78 & 0.84 & 0.05 & 1681 & 4080 & 108 & 0.00089 & 0.00973 & 1296 & 142.4 \\
\hline 0.52 & 126 & 3.78 & 0.85 & 0.03 & 1667 & 4080 & 108.4 & 0.00089 & 0.00983 & 1305 & 142.1 \\
\hline 2 & 253 & 3.78 & 0.84 & 0.06 & 1711 & 4080 & 107.1 & 0.0009 & 0.00953 & 1278 & 143 \\
\hline 1.95 & 251 & 3.78 & 0.87 & 0.1 & 1694 & 4080 & 107.6 & 0.00089 & 0.00965 & 1288 & 142.7 \\
\hline 3.67 & 394 & 3.78 & 0.85 & 0.09 & 1663 & 4080 & 108.5 & 0.00089 & 0.00986 & 1308 & 142.1 \\
\hline 4.14 & 395 & 3.78 & 0.76 & 0.05 & 1627 & 4080 & 109.5 & 0.00089 & 0.01011 & 1331 & 141.3 \\
\hline 1.22 & 150 & 3.78 & 0.84 & 0.03 & 1636 & 4080 & 109.3 & 0.00089 & 0.01004 & 1326 & 141.5 \\
\hline 1.11 & 149 & 3.78 & 0.86 & 0.04 & 1630 & 4080 & 109.4 & 0.00089 & 0.01009 & 1329 & 141.4 \\
\hline 3.08 & 249 & 3.78 & 0.92 & 0.07 & 1714 & 4080 & 107 & 0.0009 & 0.00951 & 1276 & 143.1 \\
\hline 3.03 & 248 & 3.78 & 0.94 & 0.16 & 1730 & 4080 & 106.6 & 0.0009 & 0.00941 & 1266 & 143.4 \\
\hline 7.8 & 393 & 3.78 & 0.86 & 0.05 & 1672 & 4080 & 108.2 & 0.00089 & 0.00979 & 1302 & 142.2 \\
\hline 7.57 & 392 & 3.78 & 0.86 & 0.05 & 1671 & 4080 & 108.2 & 0.00089 & 0.0098 & 1303 & 142.2 \\
\hline 16.72 & 599 & 3.78 & 0.85 & 0.07 & 1647 & 4080 & 108.9 & 0.00089 & 0.00997 & 1318 & 141.7 \\
\hline 16.45 & 598 & 3.78 & 0.85 & 0.08 & 1651 & 4080 & 108.8 & 0.00089 & 0.00994 & 1316 & 141.8 \\
\hline
\end{tabular}

R507a

\begin{tabular}{|c|c|c|c|c|c|c|c|c|c|c|c|}
\hline 0.63 & 128 & 3.78 & 0.886 & 0.005 & 1923 & 3790 & 114.4 & 0.00104 & 0.00901 & 1086 & 140.6 \\
\hline 0.55 & 129 & 3.78 & 0.861 & 0.058 & 2434 & 3790 & 100.2 & 0.00111 & 0.00696 & 963 & 149.4 \\
\hline 2.93 & 258 & 3.78 & 0.843 & 0.097 & 1927 & 3790 & 114.3 & 0.00104 & 0.00898 & 1085 & 140.6 \\
\hline 2.71 & 258 & 3.78 & 0.844 & 0.111 & 1934 & 3790 & 114 & 0.00104 & 0.00894 & 1083 & 140.8 \\
\hline 4.11 & 248 & 3.78 & 0.856 & 0.48 & 1916 & 3790 & 114.7 & 0.00104 & 0.00905 & 1088 & 140.4 \\
\hline 3.82 & 250 & 3.78 & 0.759 & 0.237 & 1905 & 3790 & 115 & 0.00104 & 0.00912 & 1091 & 140.2 \\
\hline 2.57 & 252 & 3.78 & 0.566 & 0.018 & 1890 & 3790 & 115.1 & 0.00104 & 0.00921 & 1095 & 139.9 \\
\hline 2.46 & 256 & 3.78 & 0.858 & 0.073 & 2394 & 3790 & 98 & 0.00112 & 0.00671 & 939 & 150.9 \\
\hline 2.26 & 255 & 3.78 & 0.873 & 0.303 & 2364 & 3790 & 99.1 & 0.00111 & 0.00683 & 951 & 150.1 \\
\hline 2.81 & 255 & 3.78 & 0.773 & 0.284 & 2346 & 3790 & 99.8 & 0.00111 & 0.00691 & 958 & 149.7 \\
\hline 1.86 & 253 & 3.78 & 0.5 & 0.015 & 2355 & 3790 & 99.4 & 0.00111 & 0.00687 & 954 & 149.9 \\
\hline $\begin{array}{l}6.00 \\
6\end{array}$ & 397 & 3.78 & 0.875 & 0.029 & 1928 & 3790 & 114.2 & 0.00104 & 0.00898 & 1084 & 140.7 \\
\hline 6 & 397 & 3.78 & 0.875 & 0.029 & 1928 & 3790 & 114.2 & 0.00104 & 0.00898 & 1084 & 140.7 \\
\hline 7.93 & 405 & 3.78 & 0.86 & 0.261 & 1880 & 3790 & 115.9 & 0.00104 & 0.00927 & 1098 & 139.7 \\
\hline 6.86 & 390 & 3.78 & 0.739 & 0.226 & 1870 & 3790 & 116.1 & 0.00104 & 0.00933 & 1101 & 139.5 \\
\hline 4.39 & 401 & 3.78 & 0.425 & 0.032 & 1890 & 3790 & 115.5 & 0.00104 & 0.00921 & 1095 & 139.9 \\
\hline 4.91 & 396 & 3.78 & 0.854 & 0.059 & 2356 & 3790 & 99.4 & 0.00111 & 0.00686 & 954 & 149.9 \\
\hline 5.77 & 396 & 3.78 & 0.844 & 0.38 & 2363 & 3790 & 99.2 & 0.00111 & 0.00684 & 951 & 150.1 \\
\hline 5.51 & 396 & 3.78 & 0.797 & 0.209 & 2399 & 3790 & 97.9 & 0.00112 & 0.00669 & 937 & 151 \\
\hline 3.97 & 397 & 3.78 & 0.59 & 0.066 & 2366 & 3790 & 99.1 & 0.00111 & 0.00682 & 950 & 150.2 \\
\hline 11.93 & 608 & 3.78 & 0.843 & 0.024 & 1896 & 3790 & 115.3 & 0.00104 & 0.00917 & 1093 & 140 \\
\hline 11.78 & 607 & 3.78 & 0.838 & 0.035 & 1897 & 3790 & 115.3 & 0.00104 & 0.00916 & 1093 & 140 \\
\hline 16.62 & 601 & 3.78 & 0.845 & 0.394 & 1906 & 3790 & 114.9 & 0.00104 & 0.00911 & 1090 & 140.2 \\
\hline 13.6 & 595 & 3.78 & 0.684 & 0.281 & 1894 & 3790 & 115.4 & 0.00104 & 0.00918 & 1094 & 140 \\
\hline 8.42 & 598 & 3.78 & 0.45 & 0.054 & 1901 & 3790 & 115.1 & 0.00104 & 0.00914 & 1092 & 140.1 \\
\hline 8.54 & 608 & 3.78 & 0.851 & 0.044 & 2408 & 3790 & 97.5 & 0.00112 & 0.00665 & 934 & 151.2 \\
\hline 10.46 & 593 & 3.78 & 0.88 & 0.391 & 2416 & 3790 & 97.3 & 0.00112 & 0.00662 & 931 & 151.4 \\
\hline 10.56 & 598 & 3.78 & 0.742 & 0.291 & 2385 & 3790 & 98.3 & 0.00112 & 0.00675 & 943 & 150.6 \\
\hline 6.65 & 592 & 3.78 & 0.421 & 0.04 & 2384 & 3790 & 98.2 & 0.00112 & 0.00675 & 943 & 150.6 \\
\hline 0.66 & 127 & 3.78 & 0.884 & 0.068 & 1670 & 3790 & 122.9 & 0.00101 & 0.01072 & 1163 & 135.6 \\
\hline 0.41 & 127 & 3.78 & 0.883 & 0.069 & 1670 & 3790 & 122.9 & 0.00101 & 0.01072 & 1163 & 135.6 \\
\hline 1.5 & 242 & 3.78 & 0.87 & 0.082 & 1670 & 3790 & 122.9 & 0.00101 & 0.01072 & 1163 & 135.6 \\
\hline 1.51 & 243 & 3.78 & 0.871 & 0.078 & 1670 & 3790 & 122.9 & 0.00101 & 0.01072 & 1163 & 135.6 \\
\hline 3.67 & 394 & 3.78 & 0.85 & 0.09 & 1663 & 3790 & 123.2 & 0.00101 & 0.01077 & 1165 & 135.5 \\
\hline 4.14 & 395 & 3.78 & 0.76 & 0.05 & 1627 & 3790 & 124.4 & 0.001 & 0.01106 & 1177 & 134.8 \\
\hline 1.11 & 135 & 3.78 & 0.843 & 0.121 & 1670 & 3790 & 122.9 & 0.00101 & 0.01072 & 1163 & 135.6 \\
\hline 0.77 & 135 & 3.78 & 0.836 & 0.121 & 1670 & 3790 & 122.9 & 0.00101 & 0.01072 & 1163 & 135.6 \\
\hline 3.5 & 246 & 3.78 & 0.855 & 0.076 & 1670 & 3790 & 122.9 & 0.00101 & 0.01072 & 1163 & 135.6 \\
\hline 3.5 & 246 & 3.78 & 0.855 & 0.076 & 1670 & 3790 & 122.9 & 0.00101 & 0.01072 & 1163 & 135.6 \\
\hline 7.92 & 401 & 3.78 & 0.869 & 0.118 & 1670 & 3790 & 122.9 & 0.00101 & 0.01072 & 1163 & 135.6 \\
\hline 7.85 & 401 & 3.78 & 0.867 & 0.118 & 1670 & 3790 & 122.9 & 0.00101 & 0.01072 & 1163 & 135.6 \\
\hline 15.27 & 600 & 3.78 & 0.899 & 0.109 & 1670 & 3790 & 122.9 & 0.00101 & 0.01072 & 1163 & 135.6 \\
\hline $\begin{array}{l}14.99 \\
\text {. }\end{array}$ & 600 & 3.78 & 0.892 & 0.106 & 1670 & 3790 & 122.9 & 0.00101 & 0.01072 & 1163 & 135.6 \\
\hline
\end{tabular}




\section{Micro-fin 5/8 inch}

R22

\begin{tabular}{|c|c|c|c|c|c|c|c|c|c|c|c|}
\hline 0.61 & 126 & 3.78 & 0.91 & 0.1 & 1529 & 4990 & 164.2 & 0.00089 & 0.0149 & 1465 & 137.8 \\
\hline 0.78 & 126 & 3.78 & 0.91 & 0.1 & 1534 & 4990 & 164 & 0.00089 & 0.01485 & 1463 & 137.8 \\
\hline 3.2 & 268 & 3.78 & 0.88 & 0.1 & 1529 & 4990 & 164.2 & 0.00089 & 0.0149 & 1465 & 137.8 \\
\hline $\begin{array}{l}3.02 \\
3.02\end{array}$ & 268 & 3.78 & 0.89 & 0.1 & 1530 & 4990 & 164.2 & 0.00089 & 0.01489 & 1464 & 137.8 \\
\hline 5.54 & 398 & 3.78 & 0.75 & 0.09 & 1539 & 4990 & 163.9 & 0.00089 & 0.0148 & 1460 & 137.9 \\
\hline 5.16 & 398 & 3.78 & 0.75 & 0.08 & 1536 & 4990 & 164 & 0.00089 & 0.01483 & 1462 & 137.9 \\
\hline \multicolumn{12}{|c|}{ R134a } \\
\hline 0.92 & 124 & 3.78 & 0.88 & 0.09 & 1009 & 4067 & 163.5 & 0.00087 & 0.02015 & 1711 & 128.3 \\
\hline 0.96 & 124 & 3.78 & 0.88 & 0.09 & 1010 & 4067 & 163.5 & 0.00087 & 0.02013 & 1711 & 128.4 \\
\hline 0.86 & 125 & 3.78 & 0.86 & 0.05 & 1006 & 4067 & 163.6 & 0.00087 & 0.02021 & 1714 & 128.3 \\
\hline 3.35 & 259 & 3.78 & 0.92 & 0.1 & 1010 & 4067 & 163.5 & 0.00087 & 0.02013 & 1711 & 128.4 \\
\hline 3.38 & 259 & 3.78 & 0.91 & 0.1 & 1007 & 4067 & 163.6 & 0.00087 & 0.02019 & 1713 & 128.3 \\
\hline 3.83 & 252 & 3.78 & 0.87 & 0.08 & 1001 & 4067 & 163.9 & 0.00087 & 0.02032 & 1718 & 128.2 \\
\hline 3.15 & 252 & 3.78 & 0.87 & 0.08 & 1002 & 4067 & 163.8 & 0.00087 & 0.0203 & 1717 & 128.2 \\
\hline 5.63 & 348 & 3.78 & 0.89 & 0.1 & 1022 & 4067 & 163 & 0.00087 & 0.01988 & 1700 & 128.6 \\
\hline 5.77 & 351 & 3.78 & 0.87 & 0.1 & 1020 & 4067 & 163.1 & 0.00087 & 0.01992 & 1702 & 128.6 \\
\hline 4.81 & 322 & 3.78 & 0.87 & 0.05 & 1017 & 4067 & $\begin{array}{l}163.2 \\
\end{array}$ & 0.00087 & 0.01998 & 1705 & 128.5 \\
\hline 4.61 & 323 & 3.78 & 0.88 & 0.04 & 1020 & 4067 & 163.1 & 0.00087 & 0.01992 & 1702 & 128.6 \\
\hline \multicolumn{12}{|c|}{$\mathrm{R} 410 \mathrm{~A}$} \\
\hline 0.59 & 125 & 3.78 & 0.86 & 0.06 & 2448 & 4950 & 165.7 & 0.00102 & 0.00991 & 993.3 & 143 \\
\hline 0.64 & 125 & 3.78 & 0.86 & 0.06 & 2452 & 4950 & 165.5 & 0.00102 & 0.00989 & 992.4 & 143 \\
\hline 1.73 & 250 & 3.78 & 0.88 & 0.08 & 2470 & 4950 & 165 & 0.00102 & 0.00981 & 988.2 & 143.3 \\
\hline 1.5 & 250 & 3.78 & 0.88 & 0.08 & 2471 & 4950 & 164.9 & 0.00102 & 0.0098 & 987.9 & 143.3 \\
\hline 3.44 & 400 & 3.78 & 0.68 & 0.1 & 2391 & 4950 & 167.6 & 0.00101 & 0.01021 & 1007 & 142.2 \\
\hline 3.84 & 400 & 3.78 & 0.68 & 0.11 & 2402 & 4950 & 167.2 & 0.00101 & 0.01015 & 1004 & 142.3 \\
\hline \multicolumn{12}{|c|}{ R502 } \\
\hline 0.58 & 126 & 3.78 & 0.84 & 0.05 & 1681 & 4080 & 108 & 0.00089 & 0.00973 & 1296 & 142.4 \\
\hline 0.52 & 126 & 3.78 & 0.85 & 0.03 & 1667 & 4080 & 108.4 & 0.00089 & 0.00983 & 1305 & 142.1 \\
\hline 2 & 253 & 3.78 & 0.84 & 0.06 & 1711 & 4080 & 107.1 & 0.0009 & 0.00953 & 1278 & 143 \\
\hline 1.95 & 251 & 3.78 & 0.87 & 0.1 & 1694 & 4080 & 107.6 & 0.00089 & 0.00965 & 1288 & 142.7 \\
\hline 3.67 & 394 & 3.78 & 0.85 & 0.09 & 1663 & 4080 & 108.5 & 0.00089 & 0.00986 & 1308 & 142.1 \\
\hline 4.14 & 395 & 3.78 & 0.76 & 0.05 & 1627 & 4080 & 109.5 & 0.00089 & 0.01011 & 1331 & 141.3 \\
\hline \multicolumn{12}{|c|}{ R507a } \\
\hline 0.66124 & 127.46 & 3.78 & 0.88442 & 0.0677 & 1670 & 3790 & 122.9 & 0.00101 & 0.01072 & 1163 & 135.6 \\
\hline 0.40981 & 127.43 & 3.78 & 0.88302 & 0.0692 & 1670 & 3790 & 122.9 & 0.00101 & 0.01072 & 1163 & 135.6 \\
\hline 1.5023 & 242.35 & 3.78 & 0.86959 & 0.082 & 1670 & 3790 & 122.9 & 0.00101 & 0.01072 & 1163 & 135.6 \\
\hline 1.5144 & 242.56 & 3.78 & 0.87124 & 0.0782 & 1670 & 3790 & 122.9 & 0.00101 & 0.01072 & 1163 & 135.6 \\
\hline 3.67 & 394 & 3.78 & 0.85 & 0.09 & 1663 & 3790 & 123.2 & 0.00101 & 0.01077 & 1165 & 135.5 \\
\hline 4.14 & 395 & 3.78 & 0.76 & 0.05 & 1627 & 3790 & 124.4 & 0.001 & 0.01106 & 1177 & 134.8 \\
\hline
\end{tabular}

\section{Micro-fin 5/16 inch}

\section{R22}

\begin{tabular}{|c|c|c|c|c|c|c|c|c|c|c|c|}
\hline 1.44 & 125 & 3.78 & 0.84 & 0.11 & 1534 & 4990 & 164 & 0.00089 & 0.01485 & 1463 & 137.8 \\
\hline 1.44 & 125 & 3.78 & 0.84 & 0.13 & 1538 & 4990 & 163.9 & 0.00089 & 0.01481 & 1461 & 137.9 \\
\hline 5.32 & 252 & 3.78 & 0.89 & 0.15 & 1537 & 4990 & 163.9 & 0.00089 & 0.01482 & 1461 & 137.9 \\
\hline 5.82 & 251 & 3.78 & 0.89 & 0.17 & 1530 & 4990 & 164.2 & 0.00089 & 0.01489 & 1464 & 137.8 \\
\hline 12.52 & 407 & 3.78 & 0.89 & 0.09 & 1554 & 4990 & 163.4 & 0.00089 & 0.01464 & 1454 & 138.2 \\
\hline 12.73 & 406 & 3.78 & 0.9 & 0.09 & 1554 & 4990 & 163.4 & 0.00089 & 0.01464 & 1454 & 138.2 \\
\hline 28.42 & 610 & 3.78 & 0.85 & 0.12 & 1504 & 4990 & 165 & 0.00088 & 0.01517 & 1476 & 137.3 \\
\hline 28.25 & 611 & 3.78 & 0.85 & 0.11 & 1499 & 4990 & 165.1 & 0.00088 & 0.01523 & 1479 & 137.3 \\
\hline \multicolumn{12}{|c|}{ R134a } \\
\hline 1.64 & 128 & 3.78 & 0.91 & 0.04 & 1013 & 4067 & 163.4 & 0.00087 & 0.02007 & 1708 & 128.4 \\
\hline 1.72 & 128 & 3.78 & 0.9 & 0.05 & 1014 & 4067 & 163.4 & 0.00087 & 0.02004 & 1707 & 128.4 \\
\hline 6.26 & 241 & 3.78 & 0.89 & 0.08 & 1017 & 4067 & 163.2 & 0.00087 & 0.01998 & 1705 & 128.5 \\
\hline 6.55 & 241 & 3.78 & 0.89 & 0.07 & 1017 & 4067 & 163.2 & 0.00087 & 0.01998 & 1705 & 128.5 \\
\hline 7.52 & 254 & 3.78 & 0.86 & 0.1 & 1001 & 4067 & 163.9 & 0.00087 & 0.02032 & 1718 & 128.2 \\
\hline 7.55 & 253 & 3.78 & 0.86 & 0.1 & 1001 & 4067 & 163.9 & 0.00087 & 0.02032 & 1718 & 128.2 \\
\hline 15.97 & 388 & 3.78 & 0.87 & 0.08 & 1007 & 4067 & 163.6 & 0.00087 & 0.02019 & 1713 & 128.3 \\
\hline $\begin{array}{l}16.49 \\
\end{array}$ & 387 & 3.78 & 0.87 & $\begin{array}{l}0.08 \\
0.08\end{array}$ & 1004 & 4067 & $\begin{array}{l}163.0 \\
163.7\end{array}$ & 0.00087 & 0.02026 & 1716 & $\begin{array}{l}128.2 \\
128.2\end{array}$ \\
\hline 17.59 & 396 & 3.78 & 0.87 & 0.11 & 995 & 4067 & 164.1 & 0.00087 & 0.02045 & 1724 & 128.1 \\
\hline 16.61 & 398 & 3.78 & $\begin{array}{l}0.07 \\
0.9\end{array}$ & 0.08 & 1017 & 4067 & 163.2 & 0.00087 & $\begin{array}{l}0.0201998 \\
0.01998\end{array}$ & 1705 & 128.5 \\
\hline 35.35 & 593 & 3.78 & 0.89 & 0.1 & 1010 & 4067 & 163.5 & 0.00087 & 0.02013 & 1711 & 128.4 \\
\hline 35.04 & 592 & 3.78 & $\begin{array}{l}0.89 \\
0.89\end{array}$ & $\begin{array}{l}0.1 \\
0.1\end{array}$ & 1010 & 4067 & 163.5 & 0.00087 & 0.02013 & 1711 & $\begin{array}{l}128.4 \\
128.4\end{array}$ \\
\hline 52.89 & 791 & 3.78 & 0.88 & 0.09 & 1012 & 4067 & 163.4 & 0.00087 & 0.02009 & 1709 & 128.4 \\
\hline 53.46 & 791 & 3.78 & 0.87 & 0.08 & 1013 & 4067 & 163.4 & 0.00087 & 0.02007 & 1708 & 128.4 \\
\hline \multicolumn{12}{|c|}{ R410A } \\
\hline 0.76 & 127 & 3.78 & 0.82 & 0.1 & 2452 & 4950 & 165.5 & 0.00102 & 0.00989 & 992.4 & 143 \\
\hline 0.98 & 127 & 3.78 & 0.84 & 0.16 & 2455 & 4950 & 165.5 & 0.00102 & 0.00988 & 991.7 & 143.1 \\
\hline 3.19 & 250 & 3.78 & 0.89 & 0.08 & 2446 & 4950 & 165.7 & 0.00102 & 0.00993 & 993.8 & 143 \\
\hline 2.89 & 250 & 3.78 & 0.83 & 0.05 & 2464 & 4950 & 165.2 & 0.00102 & 0.00984 & 989.6 & 143.2 \\
\hline 7.6 & 406 & 3.78 & 0.85 & 0.03 & 2451 & 4950 & 165.6 & 0.00102 & 0.0099 & 992.6 & 143 \\
\hline 7.11 & 405 & 3.78 & 0.85 & 0.04 & 2464 & 4950 & $\begin{array}{l}165.0 \\
165.2\end{array}$ & 0.00102 & 0.00984 & 989.6 & 143.2 \\
\hline 15.67 & 597 & 3.78 & 0.88 & 0.06 & 2438 & 4950 & 166 & 0.00102 & 0.00997 & 995.7 & 142.8 \\
\hline 15.42 & 597 & 3.78 & 0.88 & 0.06 & 2429 & 4950 & 166.4 & 0.00102 & 0.01001 & 997.9 & 142.7 \\
\hline \multicolumn{12}{|c|}{ R502 } \\
\hline 1.22 & 150 & 3.78 & 0.84 & 0.03 & 1636 & 4080 & 109.3 & 0.00089 & 0.01004 & 1326 & 141.5 \\
\hline $\begin{array}{l}1.11 \\
11\end{array}$ & 149 & 3.78 & 0.86 & 0.04 & 1630 & 4080 & 109.4 & 0.00089 & 0.01009 & 1329 & 141.4 \\
\hline 3.08 & 249 & 3.78 & 0.92 & 0.07 & 1714 & 4080 & 107 & 0.0009 & 0.00951 & 1276 & 143.1 \\
\hline $\begin{array}{l}3.03 \\
3.03\end{array}$ & 248 & 3.78 & 0.94 & 0.16 & 1730 & 4080 & 106.6 & 0.0009 & 0.00941 & 1266 & 143.4 \\
\hline 7.8 & 393 & 3.78 & 0.86 & 0.05 & 1672 & 4080 & 108.2 & 0.00089 & 0.00979 & 1302 & 142.2 \\
\hline 7.57 & 392 & 3.78 & 0.86 & 0.05 & 1671 & 4080 & 108.2 & 0.00089 & 0.0098 & 1303 & 142.2 \\
\hline 16.72 & 599 & 3.78 & 0.85 & 0.07 & 1647 & 4080 & 108.9 & 0.00089 & 0.00997 & 1318 & 141.7 \\
\hline $\begin{array}{l}16.42 \\
16.45\end{array}$ & 598 & 3.78 & 0.85 & 0.08 & 1651 & 4080 & 108.8 & 0.00089 & 0.00994 & 1316 & 141.8 \\
\hline \multicolumn{12}{|c|}{ R507 } \\
\hline 1.1132 & 134.51 & 3.78 & 0.84307 & 0.12052 & 1670 & 3790 & 122.9 & 0.00101 & 0.01072 & 1163 & 135.6 \\
\hline 0.76528 & 134.63 & 3.78 & 0.83554 & 0.12114 & 1670 & 3790 & 122.9 & 0.00101 & 0.01072 & 1163 & 135.6 \\
\hline $\begin{array}{l}0.1652 \\
1.951\end{array}$ & $\begin{array}{l}134.63 \\
246.46\end{array}$ & $\begin{array}{l}3.18 \\
3.78\end{array}$ & $\begin{array}{l}0.83534 \\
0.85469\end{array}$ & $\begin{array}{l}0.12114 \\
0.07589\end{array}$ & $\begin{array}{l}1670 \\
1670\end{array}$ & 3790 & $\begin{array}{l}122.9 \\
122.9\end{array}$ & $\begin{array}{l}0.00101 \\
0.00101\end{array}$ & $\begin{array}{l}0.010 / 2 \\
0.01072\end{array}$ & $\begin{array}{l}1103 \\
1163\end{array}$ & $\begin{array}{l}135.6 \\
135.6\end{array}$ \\
\hline 3.4951 & 246.46 & 3.78 & 0.85469 & 0.07589 & 1670 & 3790 & 122.9 & 0.00101 & 0.01072 & 1163 & 135.6 \\
\hline $\begin{array}{l}3.4951 \\
7.9245\end{array}$ & $\begin{array}{l}446.46 \\
401.37\end{array}$ & $\begin{array}{l}3.18 \\
3.78\end{array}$ & $\begin{array}{l}0.854699 \\
0.86897\end{array}$ & $\begin{array}{l}0.07589 \\
0.11843\end{array}$ & $\begin{array}{l}10 / 0 \\
1670\end{array}$ & 3790 & $\begin{array}{l}122.9 \\
122.9\end{array}$ & $\begin{array}{l}0.00101 \\
0.00101\end{array}$ & $\begin{array}{l}0.010 / 2 \\
0.01072\end{array}$ & $\begin{array}{l}1103 \\
1163\end{array}$ & $\begin{array}{l}135.6 \\
135.6\end{array}$ \\
\hline 7.8512 & 401.14 & 3.78 & 0.86676 & 0.11771 & 1670 & 3790 & 122.9 & 0.00101 & 0.01072 & 1163 & 135.6 \\
\hline $\begin{array}{l}1.80 .272 \\
152\end{array}$ & $\begin{array}{l}401.14 \\
599.58\end{array}$ & $\begin{array}{l}3.18 \\
3.78\end{array}$ & $\begin{array}{l}0.80010 \\
0.89876\end{array}$ & 0.10928 & 1670 & 3790 & $\begin{array}{l}122.9 \\
122.9\end{array}$ & 0.00101 & $\begin{array}{l}0.01072 \\
0.01072\end{array}$ & $\begin{array}{l}1103 \\
1163\end{array}$ & $\begin{array}{l}153.0 \\
135.6\end{array}$ \\
\hline
\end{tabular}




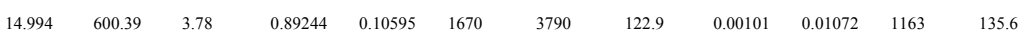




\section{APPENDIX D}

Pressure drop without oil of Iowa State University (file: iow_all.in)

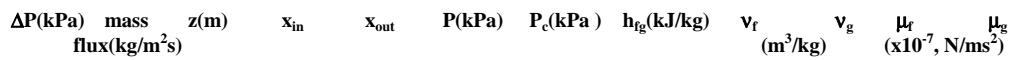

\section{Smooth 3/8 inch}

R134a

\begin{tabular}{|c|c|c|c|c|c|c|c|c|c|c|c|}
\hline 2.19 & 86 & 3.97 & 0.05 & 0.85 & 310 & 4067 & 197.4 & 0.00078 & 0.06561 & 2820 & 109.9 \\
\hline 2.15 & 86 & 3.97 & 0.05 & 0.84 & 311 & 4067 & 197.4 & 0.00078 & 0.0654 & 2816 & 109 \\
\hline 4.66 & 121 & 3.97 & 0.05 & 0.82 & 306 & 4067 & 197.7 & 0.00078 & 0.06644 & 2833 & 109.7 \\
\hline 4.55 & 121 & 3.97 & 0.05 & 0.82 & 306 & 4067 & 197.7 & 0.00078 & 0.06644 & 2833 & 109.7 \\
\hline 5.39 & 125 & 3.97 & 0.07 & 0.85 & 299 & 4067 & 198.3 & 0.00077 & 0.06794 & 2857 & 109.4 \\
\hline 13.34 & 200 & 3.97 & 0.11 & 0.82 & 305 & 4067 & 197.8 & 0.00078 & 0.06665 & 2836 & 109.7 \\
\hline 13.35 & 200 & 3.97 & 0.11 & 0.82 & 307 & 4067 & 197.7 & 0.00078 & 0.06623 & 2830 & 109.8 \\
\hline 13.48 & 201 & 3.97 & 0.11 & 0.82 & 305 & 4067 & 197.8 & 0.00078 & 0.06665 & 2836 & 109.7 \\
\hline 27.4 & 310 & 3.97 & 0.07 & 0.82 & 303 & 4067 & 198 & 0.00078 & 0.06707 & 2843 & 109.6 \\
\hline 28.06 & 308 & 3.97 & 0.07 & 0.83 & 299 & 4067 & 198.3 & 0.00077 & 0.06794 & 2857 & 109.4 \\
\hline 27.42 & 307 & 3.97 & 0.07 & 0.83 & 304 & 4067 & 197.9 & 0.00078 & 0.06686 & 2840 & 109.7 \\
\hline 39.24 & 372 & 3.97 & 0.1 & 0.8 & 310 & 4067 & 197.4 & 0.00078 & 0.06561 & 2820 & 109.9 \\
\hline 38.72 & 362 & 3.97 & 0.11 & 0.83 & 310 & 4067 & 197.4 & 0.00078 & 0.06561 & 2820 & 109.9 \\
\hline 0.18 & 87 & 3.97 & 0.85 & 0.15 & 1008 & 4067 & 163.6 & 0.00087 & 0.02017 & 1712 & 128 \\
\hline 0.21 & 87 & 3.97 & 0.85 & 0.15 & 1008 & 4067 & 163.6 & 0.00087 & 0.02017 & 1712 & 128 \\
\hline 0.56 & 121 & 3.97 & 0.85 & 0.15 & 1003 & 4067 & 163.8 & 0.00087 & 0.02028 & 1717 & 128 \\
\hline 0.62 & 121 & 3.97 & 0.85 & 0.14 & 1002 & 4067 & 163.8 & 0.00087 & 0.0203 & 1717 & 128 \\
\hline 2.37 & 206 & 3.97 & 0.86 & 0.15 & 992 & 4067 & 164.2 & 0.00087 & 0.02051 & 1726 & 128 \\
\hline 2.59 & 206 & 3.97 & 0.86 & 0.15 & 990 & 4067 & 164.3 & 0.00087 & 0.02056 & 1728 & 128 \\
\hline 5.3 & 305 & 3.97 & 0.83 & 0.16 & 975 & 4067 & 164.8 & 0.00087 & 0.02089 & 1741 & 128 \\
\hline 5.53 & 305 & 3.97 & 0.82 & 0.16 & 976 & 4067 & 164.8 & 0.00087 & 0.02087 & 1740 & 128 \\
\hline 8.94 & 368 & 3.97 & 0.84 & 0.14 & 996 & 4067 & 164 & 0.00087 & 0.02043 & 1723 & 128 \\
\hline 8.63 & 373 & 3.97 & 0.82 & 0.14 & 993 & 4067 & 164.1 & 0.00087 & 0.02049 & 1725 & 128 \\
\hline 6.16 & 128 & 3.97 & 0.1 & 0.85 & 301 & 4067 & 198.1 & 0.00077 & 0.0675 & 2850 & 109.5 \\
\hline 6.18 & 130 & 3.97 & 0.1 & 0.84 & 301 & 4067 & 198.1 & 0.00077 & 0.0675 & 2850 & 109.5 \\
\hline 12.29 & 191 & 3.97 & 0.08 & 0.81 & 306 & 4067 & 197.7 & 0.00078 & 0.06644 & 2833 & 109.7 \\
\hline 11.74 & 188 & 3.97 & 0.08 & 0.8 & 307 & 4067 & 197.7 & 0.00078 & 0.06623 & 2830 & 109.8 \\
\hline 27.45 & 294 & 3.97 & 0.09 & 0.84 & 304 & 4067 & 197.9 & 0.00078 & 0.06686 & 2840 & 109.7 \\
\hline 26.44 & 290 & 3.97 & 0.1 & 0.87 & 298 & 4067 & 198.3 & 0.00077 & 0.06816 & 2860 & 109.4 \\
\hline 39.57 & 365 & 3.97 & 0.11 & 0.81 & 307 & 4067 & 197.7 & 0.00078 & 0.06623 & 2830 & 109.8 \\
\hline 40.01 & 360 & 3.97 & 0.12 & 0.85 & 302 & 4067 & 198 & 0.00077 & 0.06728 & 2847 & 109.6 \\
\hline
\end{tabular}

\section{R12}

$\begin{array}{llllllllllll}5.26 & 132 & 3.97 & 0.09 & 0.8 & 321 & 4120 & 151.6 & 0.00072 & 0.05357 & 2436 & 114 \\ 4.26 & 133 & 3.97 & 0.09 & 0.79 & 334 & 4120 & 150.9 & 0.00072 & 0.05158 & 2403 & 115 \\ 4.47 & 133 & 3.97 & 0.09 & 0.79 & 330 & 4120 & 151.2 & 0.00072 & 0.05217 & 2413 & 1145 \\ 10.43 & 197 & 3.97 & 0.09 & 0.8 & 306 & 4120 & 152.3 & 0.00072 & 0.05608 & 2476 & 114 \\ 10.2 & 197 & 3.97 & 0.1 & 0.78 & 305 & 4120 & 152.3 & 0.00072 & 0.05626 & 2479 & 113 \\ 10.69 & 199 & 3.97 & 0.1 & 0.78 & 304 & 4120 & 152.4 & 0.00072 & 0.05643 & 2482 & 113 \\ 27.29 & 305 & 3.97 & 0.1 & 0.81 & 302 & 4120 & 152.5 & 0.00072 & 0.05679 & 2487 & 113 \\ 24.89 & 314 & 3.97 & 0.09 & 0.8 & 304 & 4120 & 152.4 & 0.00072 & 0.05643 & 2482 & 113 \\ 25.33 & 307 & 3.97 & 0.1 & 0.82 & 303 & 4120 & 152.5 & 0.00072 & 0.05661 & 2484 & 113 \\ 37.35 & 391 & 3.97 & 0.1 & 0.83 & 316 & 4120 & 151.8 & 0.00072 & 0.05438 & 2449 & 114 \\ 37.77 & 386 & 3.97 & 0.11 & 0.84 & 316 & 4120 & 151.8 & 0.00072 & 0.05438 & 2449 & 114 \\ 0.5 & 134 & 3.97 & 0.83 & 0.1 & 982 & 4120 & 128 & 0.0008 & 0.01776 & 1629 & 134 \\ 0.2 & 134 & 3.97 & 0.83 & 0.08 & 943 & 4120 & 129.2 & 0.0008 & 0.01852 & 1655 & 133 \\ 0.33 & 134 & 3.97 & 0.84 & 0.08 & 943 & 4120 & 129.2 & 0.0008 & 0.01852 & 1655 & 133 \\ 1.85 & 211 & 3.97 & 0.8 & 0.05 & 958 & 4120 & 128.7 & 0.0008 & 0.01822 & 1645 & 133 \\ 1.8 & 211 & 3.97 & 0.81 & 0.06 & 962 & 4120 & 128.7 & 0.0008 & 0.01814 & 1642 & 133 \\ 3.79 & 305 & 3.97 & 0.83 & 0.09 & 962 & 4120 & 128.7 & 0.0008 & 0.01814 & 1642 & 133 \\ 3.76 & 305 & 3.97 & 0.84 & 0.1 & 957 & 4120 & 128.8 & 0.0008 & 0.01824 & 1645 & 133 \\ 3.3 & 307 & 3.97 & 0.82 & 0.07 & 974 & 4120 & 128.3 & 0.0008 & 0.01791 & 1634 & 134 \\ 6.17 & 374 & 3.97 & 0.8 & 0.13 & 934 & 4120 & 129.5 & 0.0008 & 0.0187 & 1661 & 133 \\ 6.89 & 373 & 3.97 & 0.81 & 0.13 & 940 & 4120 & 129.3 & 0.0008 & 0.01858 & 1657 & 133\end{array}$

\section{R22}

$\begin{array}{llllllllllll}3.5 & 125 & 3.97 & 0.2 & 0.88 & 450 & 4990 & 204.8 & 0.00077 & 0.05154 & 2260 & 115.2 \\ 4.4 & 128 & 3.97 & 0.2 & 0.92 & 440 & 4990 & 205.3 & 0.00077 & 0.05268 & 2274 & 114.9 \\ 3.8 & 131 & 3.97 & 0.2 & 0.91 & 450 & 4990 & 204.8 & 0.00077 & 0.05154 & 2260 & 115.2 \\ 8.4 & 202 & 3.97 & 0.18 & 0.75 & 470 & 4990 & 203.8 & 0.00078 & 0.04941 & 2231 & 115.8 \\ 9.3 & 203 & 3.97 & 0.18 & 0.8 & 480 & 4990 & 203.3 & 0.00078 & 0.04841 & 2218 & 116.1 \\ 9 & 208 & 3.97 & 0.18 & 0.76 & 460 & 4990 & 204.3 & 0.00077 & 0.05046 & 2245 & 115.5 \\ 19.4 & 302 & 3.97 & 0.17 & 0.74 & 480 & 4990 & 203.3 & 0.00078 & 0.04841 & 2218 & 116.1 \\ 18 & 303 & 3.97 & 0.15 & 0.72 & 490 & 4990 & 202.8 & 0.00078 & 0.04745 & 2204 & 116.4 \\ 31.2 & 387 & 3.97 & 0.23 & 0.77 & 480 & 4990 & 203.3 & 0.00078 & 0.04841 & 2218 & 116.1 \\ 30.7 & 390 & 3.97 & 0.22 & 0.73 & 500 & 4990 & 202.3 & 0.00078 & 0.04653 & 2191 & 116.7 \\ 1 & 127 & 3.97 & 0.88 & 0.04 & 1600 & 4990 & 161.9 & 0.00089 & 0.01418 & 1433 & 138.9 \\ 1 & 130 & 3.97 & 0.86 & 0.05 & 1600 & 4990 & 161.9 & 0.00089 & 0.01418 & 1433 & 138.9 \\ 2.1 & 198 & 3.97 & 0.91 & 0.14 & 1570 & 4990 & 162.9 & 0.00089 & 0.01448 & 1447 & 138.4 \\ 2 & 201 & 3.97 & 0.9 & 0.15 & 1570 & 4990 & 162.9 & 0.00089 & 0.01448 & 1447 & 138.4 \\ 2.2 & 201 & 3.97 & 0.9 & 0.15 & 1570 & 4990 & 162.9 & 0.00089 & 0.01448 & 1447 & 138.4 \\ 3.8 & 302 & 3.97 & 0.93 & 0.09 & 1690 & 4990 & 159.1 & 0.0009 & 0.01334 & 1395 & 140.4 \\ 4.7 & 303 & 3.97 & 0.91 & 0.17 & 1510 & 4990 & 164.8 & 0.00088 & 0.01511 & 1474 & 137.4 \\ 4.1 & 303 & 3.97 & 0.91 & 0.11 & 1560 & 4990 & 163.2 & 0.00089 & 0.01458 & 1451 & 138.3 \\ 9.7 & 409 & 3.97 & 0.93 & 0.23 & 1460 & 4990 & 166.4 & 0.00088 & 0.01567 & 1497 & 136.6 \\ 8 & 410 & 3.97 & 0.92 & 0.19 & 1610 & 4990 & 161.6 & 0.00089 & 0.01408 & 1429 & 139.1\end{array}$


Micro-fin 3/8 inch

R134a

\begin{tabular}{llllllllllll}
3.33 & 85 & 3.97 & 0.08 & 0.84 & 310 & 4067 & 197.4 & 0.00078 & 0.0656 & 2820 & 110 \\
3.78 & 91 & 3.97 & 0.08 & 0.81 & 308 & 4067 & 197.6 & 0.00078 & 0.066 & 2830 & 110 \\
5.55 & 129 & 3.97 & 0.05 & 0.82 & 307 & 4067 & 197.7 & 0.00078 & 0.0662 & 2830 & 110 \\
5.43 & 130 & 3.97 & 0.05 & 0.81 & 310 & 4067 & 197.4 & 0.00078 & 0.0656 & 2820 & 110 \\
13.38 & 198 & 3.97 & 0.07 & 0.81 & 308 & 4067 & 197.6 & 0.00078 & 0.066 & 2830 & 110 \\
14.19 & 197 & 3.97 & 0.07 & 0.84 & 304 & 4067 & 197.9 & 0.00078 & 0.0669 & 2840 & 110 \\
14.66 & 200 & 3.97 & 0.1 & 0.83 & 304 & 4067 & 197.9 & 0.00078 & 0.0669 & 2840 & 110 \\
27.22 & 289 & 3.97 & 0.08 & 0.81 & 314 & 4067 & 197.2 & 0.00078 & 0.0648 & 2810 & 110 \\
30.19 & 299 & 3.97 & 0.08 & 0.82 & 313 & 4067 & 197.3 & 0.00078 & 0.065 & 2810 & 110 \\
40.06 & 367 & 3.97 & 0.07 & 0.82 & 329 & 4067 & 196.2 & 0.00078 & 0.0619 & 2760 & 111 \\
42.74 & 373 & 3.97 & 0.09 & 0.83 & 324 & 4067 & 196.6 & 0.00078 & 0.0629 & 2780 & 111 \\
44.47 & 375 & 3.97 & 0.09 & 0.81 & 317 & 4067 & 197 & 0.00078 & 0.0642 & 2800 & 110 \\
1.05 & 129 & 3.97 & 0.86 & 0.15 & 987 & 4067 & 164.4 & 0.00087 & 0.0206 & 1730 & 128 \\
1.07 & 129 & 3.97 & 0.86 & 0.14 & 985 & 4067 & 164.5 & 0.00087 & 0.0206 & 1730 & 128 \\
3.28 & 203 & 3.97 & 0.88 & 0.17 & 996 & 4067 & 164 & 0.00087 & 0.0204 & 1720 & 128 \\
3.31 & 202 & 3.97 & 0.89 & 0.15 & 1011 & 4067 & 163.4 & 0.00087 & 0.0201 & 1710 & 128 \\
8.07 & 300 & 3.97 & 0.87 & 0.14 & 983 & 4067 & 164.6 & 0.00087 & 0.0207 & 1730 & 128 \\
8.34 & 299 & 3.97 & 0.87 & 0.14 & 987 & 4067 & 164.4 & 0.00087 & 0.0206 & 1730 & 128 \\
12.4 & 363 & 3.97 & 0.84 & 0.15 & 978 & 4067 & 164.5 & 0.00087 & 0.0208 & 1740 & 128 \\
11.85 & 361 & 3.97 & 0.86 & 0.15 & 984 & 4067 & 164.5 & 0.00087 & 0.0206 & 1730 & 128 \\
\hline 1
\end{tabular}

R12

\begin{tabular}{|c|c|c|c|c|c|c|c|c|c|c|c|}
\hline \\
\hline 4.9 & 124 & 3.97 & 0.09 & 0.82 & 327 & 4125 & 151.3 & 0.00072 & 0.05263 & 2420 & 114 \\
\hline 4.95 & 125 & 3.97 & 0.09 & 0.79 & 325 & 4125 & 151.4 & 0.00072 & 0.05294 & 2430 & 114 \\
\hline 5.15 & 131 & 3.97 & 0.09 & 0.81 & 317 & 4125 & 151.7 & 0.00072 & 0.05422 & 2450 & 114 \\
\hline 12.3 & 203 & 3.97 & 0.09 & 0.83 & 321 & 4125 & 151.6 & 0.00072 & 0.05357 & 2440 & 114 \\
\hline 12.85 & 202 & 3.97 & 0.1 & 0.84 & 324 & 4125 & 151.4 & 0.00072 & 0.0531 & 2430 & 114 \\
\hline 26.62 & 308 & 3.97 & 0.08 & 0.81 & 310 & 4125 & 152.2 & 0.00072 & 0.05539 & 2470 & 114 \\
\hline 26.71 & 304 & 3.97 & 0.07 & 0.83 & 310 & 4125 & 152.2 & 0.00072 & 0.05539 & 2470 & 114 \\
\hline 35.55 & 358 & 3.97 & 0.11 & 0.82 & 334 & 4125 & 150.9 & 0.00072 & 0.05158 & 2400 & 115 \\
\hline 36.15 & 361 & 3.97 & 0.11 & 0.82 & 334 & 4125 & 150.9 & 0.00072 & 0.05158 & 2400 & 115 \\
\hline 4.9 & 124 & 3.97 & 0.09 & 0.82 & 327 & 4125 & 151.3 & 0.00072 & 0.05263 & 2420 & 114 \\
\hline 4.95 & 125 & 3.97 & 0.09 & 0.79 & 325 & 4125 & 151.4 & 0.00072 & 0.05294 & 2430 & 114 \\
\hline 5.15 & 131 & 3.97 & 0.09 & 0.81 & 317 & 4125 & 151.7 & 0.00072 & 0.05422 & 2450 & 114 \\
\hline 12.3 & 203 & 3.97 & 0.09 & 0.83 & 321 & 4125 & 151.6 & 0.00072 & 0.05357 & 2440 & 114 \\
\hline 12.85 & 202 & 3.97 & 0.1 & 0.84 & 324 & 4125 & 151.4 & 0.00072 & 0.0531 & 2430 & 114 \\
\hline 26.62 & 308 & 3.97 & 0.08 & 0.81 & 310 & 4125 & 152.2 & 0.00072 & 0.05539 & 2470 & 114 \\
\hline 26.71 & 304 & 3.97 & 0.07 & 0.83 & 310 & 4125 & 152.2 & 0.00072 & 0.05539 & 2470 & 114 \\
\hline 35.55 & 358 & 3.97 & 0.11 & 0.82 & 334 & 4125 & 150.9 & 0.00072 & 0.05158 & 2400 & 115 \\
\hline 36.15 & 361 & 3.97 & 0.11 & 0.82 & 334 & 4125 & 150.9 & 0.00072 & 0.05158 & 2400 & 115 \\
\hline 0.71 & 117 & 3.97 & 0.84 & 0.09 & 959 & 4125 & 128.7 & 0.0008 & 0.0182 & 1640 & 133 \\
\hline 0.73 & 115 & 3.97 & 0.86 & 0.09 & 958 & 4125 & 128.7 & 0.0008 & 0.01822 & 1650 & 133 \\
\hline 0.77 & 126 & 3.97 & 0.77 & 0.08 & 953 & 4125 & 129 & 0.00079 & 0.01832 & 1650 & 133 \\
\hline 3 & 205 & 3.97 & 0.87 & 0.08 & 960 & 4125 & 128.8 & 0.0008 & 0.01818 & 1640 & 133 \\
\hline 3.05 & 205 & 3.97 & 0.85 & 0.08 & 955 & 4125 & 128.9 & 0.0008 & 0.01828 & 1650 & 133 \\
\hline 5.65 & 299 & 3.97 & 0.86 & 0.1 & 932 & 4125 & 129.6 & 0.0008 & 0.01875 & 1660 & 133 \\
\hline 5.57 & 301 & 3.97 & 0.84 & 0.09 & 929 & 4125 & 129.6 & 0.00079 & 0.01881 & 1660 & 133 \\
\hline 5.79 & 302 & 3.97 & 0.83 & 0.09 & 927 & 4125 & 129.7 & 0.00079 & 0.01885 & 1670 & 133 \\
\hline 8.59 & 367 & 3.97 & 0.84 & 0.14 & 921 & 4125 & 129.9 & 0.00079 & 0.01897 & 1670 & 132 \\
\hline 0.71 & 117 & 3.97 & 0.84 & 0.09 & 959 & 4125 & 128.7 & 0.0008 & 0.0182 & 1640 & 133 \\
\hline 0.73 & 115 & 3.97 & 0.86 & 0.09 & 958 & 4125 & 128.7 & 0.0008 & 0.01822 & 1650 & 133 \\
\hline 0.77 & 126 & 3.97 & 0.77 & 0.08 & 953 & 4125 & 129 & 0.00079 & 0.01832 & 1650 & 133 \\
\hline 3 & 205 & 3.97 & 0.87 & 0.08 & 960 & 4125 & 128.8 & 0.0008 & 0.01818 & 1640 & 133 \\
\hline 3.05 & 205 & 3.97 & 0.85 & 0.08 & 955 & 4125 & 128.9 & 0.0008 & 0.01828 & 1650 & 133 \\
\hline 5.65 & 299 & 3.97 & 0.86 & 0.1 & 932 & 4125 & 129.6 & 0.0008 & 0.01875 & 1660 & 133 \\
\hline 5.57 & 301 & 3.97 & 0.84 & 0.09 & 929 & 4125 & 129.6 & 0.00079 & 0.01881 & 1660 & 133 \\
\hline 5.79 & 302 & 3.97 & 0.83 & 0.09 & 927 & 4125 & 129.7 & 0.00079 & 0.01885 & 1670 & 133 \\
\hline 8.59 & 367 & 3.97 & 0.84 & 0.14 & 921 & 4125 & 129.9 & 0.00079 & 0.01897 & 1670 & 132 \\
\hline \multicolumn{12}{|c|}{ R22 } \\
\hline 4.2 & 118 & 3.97 & 0.22 & 0.86 & 500 & 4990 & 202.3 & 0.00078 & 0.04653 & 2190 & 117 \\
\hline 4.4 & 119 & 3.97 & 0.21 & 0.82 & 510 & 4990 & 201.6 & 0.00078 & 0.04564 & 2180 & 117 \\
\hline 4.2 & 123 & 3.97 & 0.21 & 0.81 & 510 & 4990 & 201.6 & 0.00078 & 0.04564 & 2180 & 117 \\
\hline 4 & 125 & 3.97 & 0.2 & 0.8 & 510 & 4990 & 201.6 & 0.00078 & 0.04564 & 2180 & 117 \\
\hline 13.1 & 203 & 3.97 & 0.19 & 0.9 & 470 & 4990 & 203.8 & 0.00078 & 0.04941 & 2230 & 116 \\
\hline 12 & 204 & 3.97 & 0.15 & 0.82 & 500 & 4990 & 202.3 & 0.00078 & 0.04653 & 2190 & 117 \\
\hline 26.2 & 301 & 3.97 & 0.16 & 0.86 & 550 & 4990 & 200 & 0.00079 & 0.0424 & 2130 & 118 \\
\hline 26.4 & 306 & 3.97 & 0.17 & 0.85 & 550 & 4990 & 200 & 0.00079 & 0.0424 & 2130 & 118 \\
\hline 25.4 & 306 & 3.97 & 0.16 & 0.83 & 540 & 4990 & 200.4 & 0.00079 & 0.04316 & 2140 & 118 \\
\hline 26.2 & 316 & 3.97 & 0.16 & 0.8 & 550 & 4990 & 200 & 0.00079 & 0.0424 & 2130 & 118 \\
\hline 41.7 & 381 & 3.97 & 0.18 & 0.78 & 550 & 4990 & 200 & 0.00079 & 0.0424 & 2130 & 118 \\
\hline 42.5 & 394 & 3.97 & 0.18 & 0.81 & 550 & 4990 & 200 & 0.00079 & 0.0424 & 2130 & 118 \\
\hline 41.9 & 396 & 3.97 & 0.17 & 0.76 & 540 & 4990 & 200.4 & 0.00079 & 0.04316 & 2140 & 118 \\
\hline 1.1 & 121 & 3.97 & 0.9 & 0.05 & 1560 & 4990 & 163.2 & 0.00089 & 0.01458 & 1450 & 138 \\
\hline 1.2 & 122 & 3.97 & 0.9 & 0.06 & 1560 & 4990 & 163.2 & 0.00089 & 0.01458 & 1450 & 138 \\
\hline 1.1 & 126 & 3.97 & 0.87 & 0.05 & 1550 & 4990 & 163.5 & 0.00089 & 0.01468 & 1460 & 138 \\
\hline 2.6 & 190 & 3.97 & 0.9 & 0.09 & 1550 & 4990 & 163.5 & 0.00089 & 0.01468 & 1460 & 138 \\
\hline 2.9 & 190 & 3.97 & 0.89 & 0.1 & 1550 & 4990 & 163.5 & 0.00089 & 0.01468 & 1460 & 138 \\
\hline 3 & 193 & 3.97 & 0.92 & 0.06 & 1530 & 4990 & 164.2 & 0.00089 & 0.01489 & 1460 & 138 \\
\hline 3 & 193 & 3.97 & 0.9 & 0.07 & 1550 & 4990 & 163.5 & 0.00089 & 0.01468 & 1460 & 138 \\
\hline 6.7 & 284 & 3.97 & 0.9 & 0.15 & 1560 & 4990 & 163.2 & 0.00089 & 0.01458 & 1450 & 138 \\
\hline 7.1 & 292 & 3.97 & 0.93 & 0.09 & 1520 & 4990 & 164.4 & 0.00089 & 0.015 & 1470 & 138 \\
\hline 7.4 & 293 & 3.97 & 0.9 & 0.15 & 1560 & 4990 & 163.2 & 0.00089 & 0.01458 & 1450 & 138 \\
\hline
\end{tabular}




$\begin{array}{llllllllllll}11.5 & 380 & 3.97 & 0.89 & 0.13 & 1520 & 4990 & 163.2 & 0.00089 & 0.01458 & 1450 & 138 \\ 11.5 & 382 & 3.97 & 0.88 & 0.13 & 1530 & 4990 & 164.2 & 0.00089 & 0.01489 & 1460 & 138 \\ 11.4 & 383 & 3.97 & 0.89 & 0.13 & 1520 & 4990 & 163.2 & 0.00089 & 0.01458 & 1450 & 138\end{array}$

\section{Smooth 1/2 inch}

\section{R134a}

$\begin{array}{llllllllllll}2.42 & 82 & 3.97 & 0.09 & 0.83 & 305 & 4067 & 197.8 & 0.00078 & 0.06665 & 2836 & 109.7 \\ 2.03 & 82 & 3.97 & 0.09 & 0.76 & 314 & 4067 & 197.2 & 0.00078 & 0.0648 & 2807 & 110.1 \\ 5.02 & 121 & 3.97 & 0.11 & 0.79 & 316 & 4067 & 197 & 0.00078 & 0.0644 & 2800 & 110.1 \\ 5.66 & 120 & 3.97 & 0.12 & 0.86 & 309 & 4067 & 197.6 & 0.00078 & 0.06581 & 2823 & 109.9 \\ 15.57 & 199 & 3.97 & 0.09 & 0.87 & 315 & 4067 & 197.1 & 0.00078 & 0.0646 & 2803 & 110.1 \\ 16.75 & 205 & 3.97 & 0.08 & 0.84 & 314 & 4067 & 197.2 & 0.00078 & 0.0648 & 2810 & 110 \\ 23.3 & 246 & 3.97 & 0.06 & 0.87 & 319 & 4067 & 196.9 & 0.00078 & 0.06382 & 2791 & 110.3 \\ 24.19 & 267 & 3.97 & 0.04 & 0.79 & 318 & 4067 & 196.9 & 0.00078 & 0.06401 & 2794 & 110.2 \\ 0.24 & 83 & 3.97 & 0.87 & 0.16 & 1024 & 4067 & 162.9 & 0.00087 & 0.01984 & 1699 & 128.6 \\ 0.35 & 86 & 3.97 & 0.82 & 0.16 & 1014 & 4067 & 163.4 & 0.00087 & 0.02004 & 1707 & 128.4 \\ 0.32 & 124 & 3.97 & 0.86 & 0.16 & 1025 & 4067 & 162.9 & 0.00087 & 0.01982 & 1698 & 128.7 \\ 0.49 & 125 & 3.97 & 0.84 & 0.15 & 1012 & 4067 & 163.4 & 0.00087 & 0.02009 & 1709 & 128.4 \\ 1.04 & 191 & 3.97 & 0.82 & 0.12 & 1020 & 4067 & 163.1 & 0.00087 & 0.01992 & 1702 & 128.6 \\ 0.92 & 192 & 3.97 & 0.81 & 0.12 & 1021 & 4067 & 163.1 & 0.00087 & 0.0199 & 1701 & 128.6 \\ 2.22 & 279 & 3.97 & 0.82 & 0.14 & 1025 & 4067 & 162.9 & 0.00087 & 0.01982 & 1698 & 128.7 \\ 2.1 & 281 & 3.97 & 0.82 & 0.15 & 996 & 4067 & 164 & 0.00087 & 0.02043 & 1723 & 128.1\end{array}$

\section{Micro-fin 1/2 inch}

\section{R134a}

$\begin{array}{llllllllllll}2.75 & 85 & 3.97 & 0.08 & 0.84 & 320 & 4067 & 196.8 & 0.00078 & 0.06363 & 2787 & 110.3 \\ 2.58 & 85 & 3.97 & 0.08 & 0.81 & 325 & 4067 & 196.4 & 0.00078 & 0.06268 & 2772 & 110.5 \\ 7.41 & 129 & 3.97 & 0.11 & 0.84 & 312 & 4067 & 197.3 & 0.00078 & 0.0652 & 2813 & 110 \\ 7.06 & 128 & 3.97 & 0.11 & 0.82 & 318 & 4067 & 196.9 & 0.00078 & 0.06401 & 2794 & 110.2 \\ 8.75 & 131 & 3.97 & 0.12 & 0.87 & 310 & 4067 & 197.4 & 0.00078 & 0.06561 & 2820 & 109.9 \\ 7.92 & 133 & 3.97 & 0.1 & 0.79 & 323 & 4067 & 196.6 & 0.00078 & 0.06305 & 2778 & 110.4 \\ 16.01 & 184 & 3.97 & 0.09 & 0.78 & 309 & 4067 & 197.6 & 0.00078 & 0.06581 & 2823 & 109.9 \\ 16.05 & 184 & 3.97 & 0.08 & 0.79 & 304 & 4067 & 197.9 & 0.00078 & 0.06686 & 2840 & 109.7 \\ 25.17 & 245 & 3.97 & 0.09 & 0.81 & 361 & 4067 & 194.1 & 0.00078 & 0.05661 & 2666 & 111.8 \\ 25.75 & 227 & 3.97 & 0.11 & 0.89 & 351 & 4067 & 194.8 & 0.00078 & 0.05817 & 2694 & 111.5 \\ 26.04 & 259 & 3.97 & 0.08 & 0.75 & 354 & 4067 & 194.6 & 0.00078 & 0.0577 & 2685 & 111.6 \\ 0.21 & 89 & 3.97 & 0.82 & 0.09 & 1052 & 4067 & 161.9 & 0.00088 & 0.01928 & 1675 & 129.2 \\ 0.28 & 89 & 3.97 & 0.82 & 0.11 & 1044 & 4067 & 162.1 & 0.00088 & 0.01944 & 1682 & 129 \\ 0.13 & 88 & 3.97 & 0.83 & 0.12 & 1038 & 4067 & 162.3 & 0.00087 & 0.01955 & 1687 & 128.9 \\ 0.49 & 122 & 3.97 & 0.82 & 0.13 & 1060 & 4067 & 161.6 & 0.00088 & 0.01912 & 1669 & 129.4 \\ 0.32 & 116 & 3.97 & 0.89 & 0.14 & 1046 & 4067 & 162.1 & 0.00088 & 0.0194 & 1680 & 129.1 \\ 0.47 & 121 & 3.97 & 0.84 & 0.16 & 1028 & 4067 & 162.8 & 0.00087 & 0.01976 & 1695 & 128.7 \\ 1.53 & 195 & 3.97 & 0.8 & 0.17 & 1052 & 4067 & 161.9 & 0.00088 & 0.01928 & 1675 & 129.2 \\ 1.45 & 196 & 3.97 & 0.8 & 0.16 & 1065 & 4067 & 161.4 & 0.00088 & 0.01903 & 1665 & 129.5 \\ 3.13 & 286 & 3.97 & 0.8 & 0.07 & 997 & 4067 & 164 & 0.00087 & 0.02041 & 1722 & 128.1 \\ 3.05 & 284 & 3.97 & 0.84 & 0.07 & 1019 & 4067 & 163.1 & 0.00087 & 0.01994 & 1703 & 128.5\end{array}$




\section{APPENDIX E}

Pressure drop with oil of Iowa State University (file: iow_allo.in)

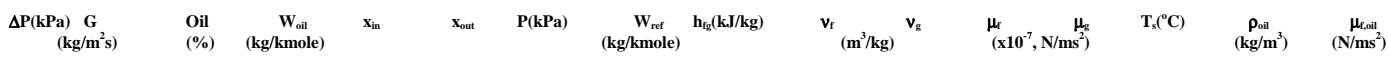

Smooth $3 / 8$ inch, condensation

R12 with SUS150 naphthenic oil

$\begin{array}{lllllll}0.67 & 129 & 1.3 & 800 & 0.78 & 0.08 & 948 \\ 0.8 & 129 & 1.3 & 800 & 0.78 & 0.08 & 940 \\ 1.89 & 208 & 1.3 & 800 & 0.83 & 0.06 & 941 \\ 1.45 & 208 & 1.3 & 800 & 0.82 & 0.06 & 938 \\ 3.41 & 293 & 1.3 & 800 & 0.83 & 0.1 & 947 \\ 3.2 & 294 & 1.3 & 800 & 0.82 & 0.1 & 944 \\ 6.24 & 367 & 1.3 & 800 & 0.81 & 0.09 & 942 \\ 6.12 & 367 & 1.3 & 800 & 0.81 & 0.09 & 942 \\ 0.37 & 130 & 2.4 & 800 & 0.82 & 0.09 & 937 \\ 0.39 & 129 & 2.4 & 800 & 0.83 & 0.09 & 935 \\ 1.47 & 198 & 2.4 & 800 & 0.85 & 0.09 & 950 \\ 1.45 & 197 & 2.4 & 800 & 0.85 & 0.09 & 950 \\ 4.74 & 297 & 2.4 & 800 & 0.82 & 0.11 & 920 \\ 4.6 & 297 & 2.4 & 800 & 0.82 & 0.11 & 922 \\ 6.89 & 368 & 2.4 & 800 & 0.82 & 0.1 & 942 \\ 6.43 & 367 & 2.4 & 800 & 0.82 & 0.1 & 944 \\ 1.31 & 123 & 4.9 & 800 & 0.81 & 0.09 & 944 \\ 1.37 & 123 & 4.9 & 800 & 0.81 & 0.09 & 940 \\ 1.45 & 197 & 4.9 & 800 & 0.8 & 0.08 & 928 \\ 1.48 & 197 & 4.9 & 800 & 0.8 & 0.08 & 925 \\ 4.05 & 303 & 4.9 & 800 & 0.79 & 0.07 & 918 \\ 4.07 & 303 & 4.9 & 800 & 0.79 & 0.07 & 916 \\ 6.07 & 363 & 4.9 & 800 & 0.82 & 0.11 & 932 \\ 6.06 & 371 & 4.9 & 800 & 0.79 & 0.1 & 926 \\ \mathbf{R} & & & \end{array}$

$\begin{array}{llllllllll}948 & 120.91 & 125.9 & 0.0007954 & 0.018 & 1654 & 132.9 & 39.3 & 952.8 & 0.0357684 \\ 940 & 120.91 & 126.1 & 0.0007945 & 0.01816 & 1660 & 132.7 & 39.2 & 952.9 & 0.0358667 \\ 941 & 120.91 & 126.2 & 0.0007946 & 0.01814 & 1659 & 132.7 & 39.2 & 952.9 & 0.0358667 \\ 938 & 120.91 & 126.2 & 0.0007942 & 0.0182 & 1661 & 132.7 & 39.1 & 952.9 & 0.0360483 \\ 947 & 120.91 & 125.9 & 0.0007952 & 0.01802 & 1655 & 132.9 & 39.5 & 952.7 & 0.0353979 \\ 944 & 120.91 & 126 & 0.0007949 & 0.01808 & 1657 & 132.8 & 39.4 & 952.8 & 0.0355869 \\ 942 & 120.91 & 126.1 & 0.0007947 & 0.01812 & 1658 & 132.8 & 39.3 & 952.8 & 0.0357684 \\ 942 & 120.91 & 126.1 & 0.0007947 & 0.01812 & 1658 & 132.8 & 39.3 & 952.8 & 0.0357684 \\ 937 & 120.91 & 126.2 & 0.0007941 & 0.01822 & 1662 & 132.6 & 39 & 953 & 0.0362375 \\ 935 & 120.91 & 126.3 & 0.0007939 & 0.01827 & 1663 & 132.6 & 39 & 953 & 0.0362375 \\ 950 & 120.91 & 125.9 & 0.0007956 & 0.01796 & 1653 & 132.9 & 39.6 & 952.6 & 0.0352089 \\ 950 & 120.91 & 125.9 & 0.0007956 & 0.01796 & 1653 & 132.9 & 39.6 & 952.6 & 0.0352089 \\ 920 & 120.91 & 126.8 & 0.0007922 & 0.01858 & 1673 & 132.2 & 38.3 & 953.4 & 0.0374496 \\ 922 & 12.91 & 126.7 & 0.0007925 & 0.01853 & 1672 & 132.3 & 38.4 & 953.4 & 0.0372678 \\ 942 & 120.91 & 126.1 & 0.0007947 & 0.01812 & 1658 & 132.8 & 39.2 & 952.9 & 0.0358667 \\ 944 & 120.91 & 126 & 0.0007949 & 0.01808 & 1657 & 132.8 & 39.3 & 952.8 & 0.0357684 \\ 944 & 120.91 & 126 & 0.0007949 & 0.01808 & 1657 & 132.8 & 39.3 & 952.8 & 0.0357684 \\ 940 & 120.91 & 126.1 & 0.0007945 & 0.01816 & 1660 & 132.7 & 39.1 & 952.9 & 0.0360483 \\ 928 & 120.91 & 126.5 & 0.0007931 & 0.01841 & 1668 & 132.4 & 38.7 & 953.2 & 0.036707 \\ 925 & 120.91 & 126.5 & 0.0007928 & 0.01847 & 1670 & 132.4 & 38.6 & 953.2 & 0.0368888 \\ 918 & 120.91 & 126.8 & 0.000792 & 0.01862 & 1675 & 132.2 & 38.3 & 953.4 & 0.0374496 \\ 916 & 120.91 & 126.8 & 0.0007918 & 0.01866 & 1676 & 132.1 & 38.2 & 953.5 & 0.0376393 \\ 932 & 120.91 & 126.3 & 0.0007936 & 0.01833 & 1665 & 132.5 & 38.8 & 953.1 & 0.0366085 \\ 926 & 120.91 & 126.6 & 0.0007929 & 0.01845 & 1669 & 132.4 & 38.6 & 953.2 & 0.0368888\end{array}$

R12 with SUS300 naphthenic oil

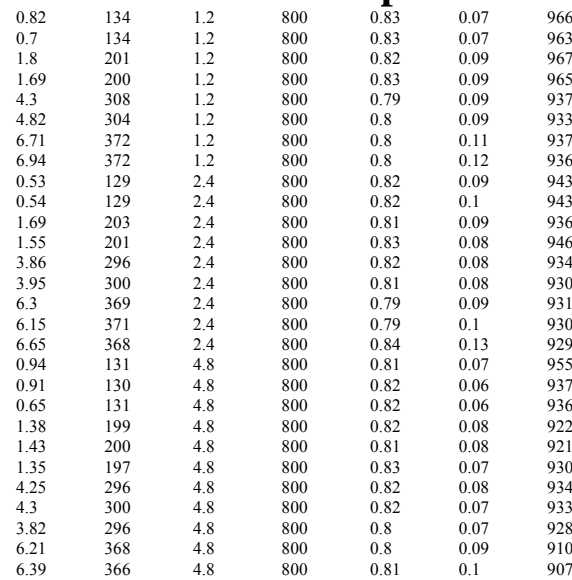
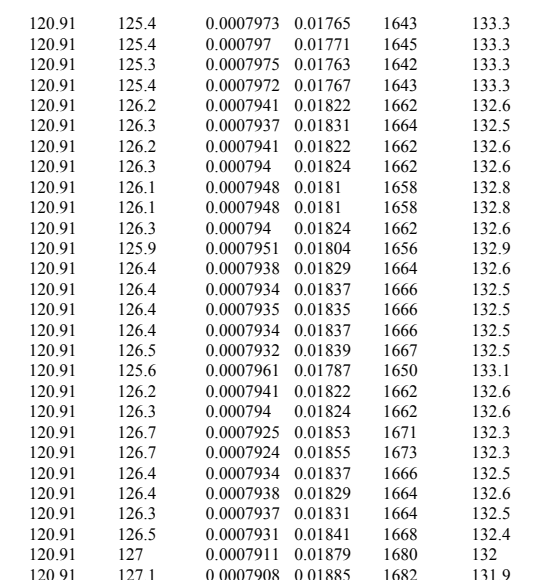

\begin{tabular}{|c|c|c|}
\hline & 953.8 & 0.0531203 \\
\hline & 953.8 & 0.0531285 \\
\hline 40.3 & & 0.0529354 \\
\hline 9. & $\begin{array}{l}953.8 \\
9544\end{array}$ & 0.0531285 \\
\hline & $\begin{array}{l}954.4 \\
9546\end{array}$ & 0.055928 \\
\hline & & $\begin{array}{l}0.0564982 \\
0.055928\end{array}$ \\
\hline & $\begin{array}{l}934.4 \\
954.5\end{array}$ & $\begin{array}{l}0.055928 \\
0.056213\end{array}$ \\
\hline & 954.3 & $\begin{array}{l}0.056213 \\
0.0554609\end{array}$ \\
\hline & 954.3 & 0.0554609 \\
\hline & $\begin{array}{r}954.5 \\
05.4\end{array}$ & 0.056213 \\
\hline & $\begin{array}{l}954.3 \\
054 .\end{array}$ & $\begin{array}{l}0.0551877 \\
0.056408\end{array}$ \\
\hline & $\begin{array}{l}954.6 \\
954.7\end{array}$ & $\begin{array}{l}0.05656982 \\
0.0569658\end{array}$ \\
\hline & 954.6 & $\begin{array}{l}0.05050776 \\
0.056716\end{array}$ \\
\hline $38.7 \quad$ & 954.7 & 0.0569658 \\
\hline 8.7 & $\begin{array}{l}954.7 \\
05.7\end{array}$ & 0.0569658 \\
\hline & $\begin{array}{l}954 \\
9544\end{array}$ & $\begin{array}{l}0.0541519 \\
0.055928\end{array}$ \\
\hline & $\begin{array}{l}954.4 \\
954.5\end{array}$ & $\begin{array}{l}0.055928 \\
0.056213\end{array}$ \\
\hline 8. & 954.9 & $\begin{array}{l}0.0578103 \\
0\end{array}$ \\
\hline & 954.9 & 0.0578103 \\
\hline & $\begin{array}{l}954.7 \\
054\end{array}$ & $\begin{array}{r}0.0569658 \\
0.053378\end{array}$ \\
\hline & $\begin{array}{l}994 \\
954.6\end{array}$ & $\begin{array}{l}0.0538789 \\
0.056498\end{array}$ \\
\hline & 954.7 & 0.0569658 \\
\hline & 955.2 & 0.0592152 \\
\hline
\end{tabular}

\section{R134a with SUS169 ester-m oil}

$\begin{array}{lllllll}0.48 & 81 & 0.5 & 500 & 0.89 & 0.11 & 1005 \\ 0.46 & 80 & 0.5 & 500 & 0.89 & 0.11 & 1007 \\ 1.69 & 126 & 0.5 & 500 & 0.87 & 0.12 & 1008 \\ 1.65 & 125 & 0.5 & 500 & 0.87 & 0.13 & 1009 \\ 2.95 & 200 & 0.5 & 500 & 0.86 & 0.12 & 1012 \\ 2.85 & 201 & 0.5 & 500 & 0.86 & 0.13 & 1013 \\ 5.78 & 301 & 0.5 & 500 & 0.85 & 0.1 & 1024 \\ 5.96 & 300 & 0.5 & 500 & 0.85 & 0.1 & 1026 \\ 8.46 & 374 & 0.5 & 500 & 0.84 & 0.11 & 1030 \\ 9.36 & 374 & 0.5 & 500 & 0.84 & 0.12 & 1020 \\ 0.15 & 83 & 1 & 500 & 0.85 & 0.11 & 1002 \\ 0.13 & 83 & 1 & 500 & 0.84 & 0.11 & 1006 \\ 0.59 & 122 & 1 & 500 & 0.9 & 0.11 & 1008 \\ 0.62 & 125 & 1 & 500 & 0.87 & 0.1 & 1012 \\ 1.91 & 204 & 1 & 500 & 0.85 & 0.09 & 1003 \\ 1.88 & 203 & 1 & 500 & 0.86 & 0.09 & 1005 \\ 4.61 & 299 & 1 & 500 & 0.84 & 0.08 & 999 \\ 4.82 & 300 & 1 & 500 & 0.83 & 0.07 & 1000 \\ 8.1 & 380 & 1 & 500 & 0.84 & 0.09 & 1001 \\ 7.86 & 380 & 1 & 500 & 0.84 & 0.09 & 1005 \\ 1.25 & 133 & 1.9 & 500 & 0.84 & 0.1 & 1037 \\ 1.06 & 132 & 1.9 & 500 & 0.85 & 0.09 & 1046 \\ 3.5 & 201 & 1.9 & 500 & 0.84 & 0.11 & 1027 \\ 3.32 & 202 & 1.9 & 500 & 0.84 & 0.11 & 1027 \\ 6.36 & 298 & 1.9 & 500 & 0.86 & 0.15 & 1012 \\ 5.64 & 299 & 1.9 & 500 & 0.84 & 0.13 & 1012 \\ 6.09 & 299 & 1.9 & 500 & 0.83 & 0.12 & 1007 \\ 10.78 & 377 & 1.9 & 500 & 0.84 & 0.14 & 1017 \\ 10 & 377 & 1.9 & 500 & 0.85 & 0.14 & 1018 \\ 9.26 & 376 & 1.9 & 500 & 0.85 & 0.14 & 1022 \\ 0.1 & 85 & 2.9 & 500 & 0.81 & 0.14 & 1008 \\ 0.1 & 85 & 2.9 & 500 & 0.8 & 0.14 & 1008 \\ 1.37 & 131 & 2.9 & 500 & 0.86 & 0.08 & 1026 \\ 1.36 & 132 & 2.9 & 500 & 0.86 & 0.1 & 1029 \\ 2.92 & 203 & 2.9 & 500 & 0.82 & 0.14 & 1028 \\ & & & & & & \\ & & & & & & \end{array}$

\begin{tabular}{|c|c|c|c|c|c|c|c|c|}
\hline 102.03 & 163.7 & 0.0008709 & 0.02023 & 1715 & 128.3 & 39.6 & 952.8 & 0.0377656 \\
\hline 102.03 & 163.6 & 0.0008711 & 0.02019 & 1713 & 128.3 & 39.7 & 952.8 & 0.0375841 \\
\hline 102.03 & 163.6 & 0.0008712 & 0.02017 & 1712 & 128.3 & 39.7 & 952.8 & 0.0375841 \\
\hline 102.03 & 163.5 & 0.0008713 & 0.02015 & 1711 & 128.3 & 39.8 & 952.7 & 0.0373947 \\
\hline 102.03 & 163.4 & 0.0008717 & 0.02009 & 1709 & 128.4 & 39.9 & 952.7 & 0.0372131 \\
\hline 102.03 & 163.4 & 0.0008718 & 0.02007 & 1708 & 128.4 & 39.9 & 952.7 & 0.0372131 \\
\hline 102.03 & 162.9 & 0.0008731 & 0.01984 & 1699 & 128.6 & 40.3 & 952.4 & 0.0365548 \\
\hline 102.03 & 162.9 & 0.0008734 & 0.0198 & 1697 & 128.7 & 40.3 & 952.4 & 0.0365548 \\
\hline 102.03 & 162.8 & 0.0008738 & 0.01972 & 1693 & 128.8 & 40.5 & 952.3 & 0.0361843 \\
\hline 102.03 & 163.1 & 0.0008726 & 0.01992 & 1702 & 128.6 & 40.1 & 952.5 & 0.0369253 \\
\hline 102.03 & 163.8 & 0.0008705 & 0.0203 & 1717 & 128.2 & 39.5 & 952.9 & 0.0379552 \\
\hline 102.03 & 163.6 & 0.000871 & 0.02021 & 1714 & 128.3 & 39.6 & 952.8 & 0.0377656 \\
\hline 102.03 & 163.6 & 0.0008712 & 0.02017 & 1712 & 128.3 & 39.7 & 952.8 & 0.0375841 \\
\hline 102.03 & 163.4 & 0.0008717 & 0.02009 & 1709 & 128.4 & 39.9 & 952.7 & 0.0372131 \\
\hline 102.03 & 163.8 & 0.0008706 & 0.02028 & 1717 & 128.2 & 39.5 & 952.9 & 0.0379552 \\
\hline 102.03 & 163.7 & 0.0008709 & 0.02023 & 1715 & 128.3 & 39.6 & 952.8 & 0.0377656 \\
\hline 102.03 & 163.9 & 0.0008702 & 0.02036 & 1720 & 128.1 & 39.3 & 953 & 0.0383264 \\
\hline 102.03 & 163.8 & 0.0008703 & 0.02034 & 1719 & 128.2 & 39.4 & 953 & 0.0381448 \\
\hline 102.03 & 163.9 & 0.0008704 & 0.02032 & 1718 & 128.2 & 39.4 & 953 & 0.0381448 \\
\hline 102.03 & 163.7 & 0.0008709 & 0.02023 & 1715 & 128.3 & 39.6 & 952.8 & 0.0377656 \\
\hline $\begin{array}{l}102.03 \\
\end{array}$ & 162.4 & 0.0008747 & 0.01957 & 1688 & 128.9 & 40.7 & 952.2 & 0.0359047 \\
\hline 102.03 & 162.1 & 0.0008757 & 0.0194 & 1680 & 129.1 & 41 & 952 & 0.0353459 \\
\hline 102.03 & 162.8 & 0.0008735 & 0.01987 & 1696 & 128.7 & 40.4 & 952.4 & 0.0363733 \\
\hline 102.03 & 162.8 & 0.0008735 & 0.01987 & 1696 & 128.7 & 40.4 & 952.4 & 0.0363733 \\
\hline 102.03 & 163.4 & 0.0008717 & 0.02009 & 1709 & 128.4 & 39.9 & 952.7 & 0.0372131 \\
\hline 102.03 & 163.4 & 0.0008717 & 0.02009 & 1709 & 128.4 & 39.9 & 952.7 & 0.0372131 \\
\hline 102.03 & 163.6 & 0.0008711 & 0.02019 & 1713 & 128.3 & 39.7 & 952.8 & 0.0375841 \\
\hline 102.03 & 163.2 & 0.0008723 & 0.01998 & 1705 & 128.5 & 40 & 952.6 & 0.0370238 \\
\hline 102.03 & 163.1 & 0.0008724 & 0.01996 & 1704 & 128.5 & 40.1 & 952.5 & 0.0369253 \\
\hline 102.03 & 163 & 0.0008729 & 0.01988 & 1700 & 128.6 & 40.2 & 952.5 & 0.0367439 \\
\hline 102.03 & 163.6 & 0.0008712 & 0.02017 & 1712 & 128.3 & 39.7 & 952.8 & 0.0375841 \\
\hline 102.03 & 163.6 & 0.0008712 & 0.02017 & 1712 & 128.3 & 39.7 & 952.8 & 0.0375841 \\
\hline 102.03 & 162.9 & 0.0008734 & 0.0198 & 1697 & 128.7 & 40.4 & 952.4 & 0.0363733 \\
\hline 102.03 & 162.7 & 0.0008737 & 0.01974 & 1694 & 128.7 & 40.4 & 952.4 & 0.0363733 \\
\hline & & & & & & & & \\
\hline
\end{tabular}



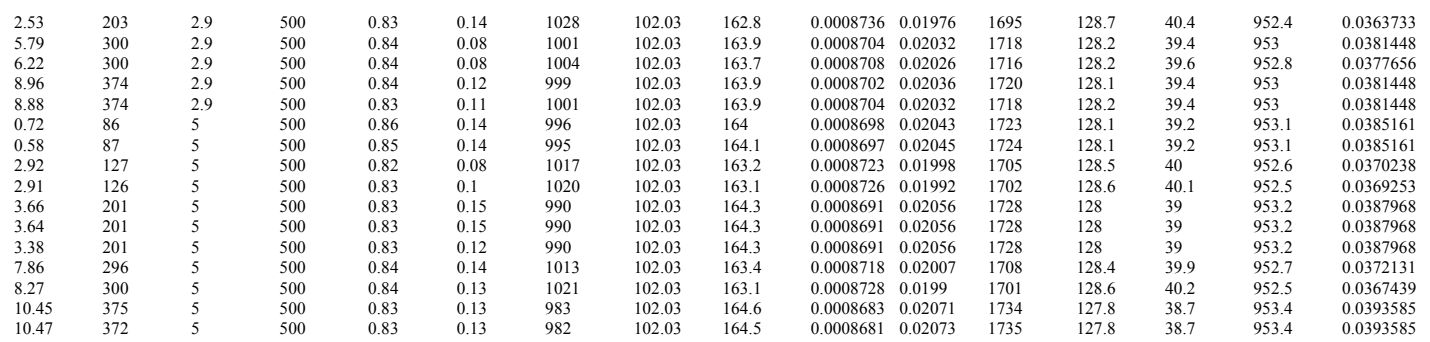

\section{R134a with SUS369 ester-m oil}

\begin{tabular}{|c|c|c|c|c|c|}
\hline & & & & & \\
\hline 0.2 & 88 & 0.6 & 500 & 0.86 & 0.15 \\
\hline 0.71 & 121 & 0.6 & 500 & 0.86 & 0.14 \\
\hline 0.74 & 123 & 0.6 & 500 & 0.86 & 0.14 \\
\hline 2.41 & 199 & 0.6 & 500 & 0.79 & 0.15 \\
\hline 2.83 & 199 & 0.6 & 500 & 0.84 & 0.15 \\
\hline 2.56 & 200 & 0.6 & 500 & 0.82 & 0.14 \\
\hline 4.96 & 296 & 0.6 & 500 & 0.84 & 0.13 \\
\hline 5.26 & 298 & 0.6 & 500 & 0.83 & 0.12 \\
\hline 8.98 & 374 & 0.6 & 500 & 0.83 & 0.13 \\
\hline 9.58 & 375 & 0.6 & 500 & 0.83 & 0.15 \\
\hline 0.05 & 89 & 1.1 & 500 & 0.86 & 0.13 \\
\hline 0.06 & 89 & 1.1 & 500 & 0.86 & 0.13 \\
\hline 0.55 & 122 & 1.1 & 500 & 0.82 & 0.13 \\
\hline 0.49 & 122 & 1.1 & 500 & 0.81 & 0.13 \\
\hline 2.92 & 203 & 1.1 & 500 & 0.83 & 0.1 \\
\hline 2.8 & 202 & 1.1 & 500 & 0.84 & 0.11 \\
\hline 6.4 & 301 & 1.1 & 500 & 0.83 & 0.12 \\
\hline 5.8 & 302 & 1.1 & 500 & 0.82 & 0.12 \\
\hline 9 & 369 & 1.1 & 500 & 0.82 & 0.13 \\
\hline 8.72 & 367 & 1.1 & 500 & 0.83 & 0.14 \\
\hline 0.41 & 87 & 2.4 & 500 & 0.83 & 0.1 \\
\hline 0.33 & 87 & 2.4 & 500 & 0.83 & 0.1 \\
\hline 0.2 & 86 & 2.4 & 500 & 0.83 & 0.11 \\
\hline 0.9 & 132 & 2.4 & 500 & 0.82 & 0.08 \\
\hline 0.8 & 131 & 2.4 & 500 & 0.83 & 0.07 \\
\hline 1.5 & 209 & 2.4 & 500 & 0.83 & 0.09 \\
\hline 1.3 & 208 & 2.4 & 500 & 0.84 & 0.09 \\
\hline 3.58 & 303 & 2.4 & 500 & 0.83 & 0.09 \\
\hline 3.58 & 299 & 2.4 & 500 & 0.85 & 0.09 \\
\hline 7.84 & 384 & 2.4 & 500 & 0.83 & 0.11 \\
\hline 7.69 & 384 & 2.4 & 500 & 0.83 & 0.12 \\
\hline 0.31 & 88 & 5 & 500 & 0.81 & 0.14 \\
\hline 0.42 & 87 & 5 & 500 & 0.81 & 0.14 \\
\hline 0.59 & 126 & 5 & 500 & 0.84 & 0.1 \\
\hline 0.36 & 126 & 5 & 500 & 0.85 & 0.1 \\
\hline 1.77 & 194 & 5 & 500 & 0.81 & 0.08 \\
\hline 1.82 & 194 & 5 & 500 & 0.82 & 0.08 \\
\hline 4.32 & 301 & 5 & 500 & 0.8 & 0.13 \\
\hline 4.59 & 301 & 5 & 500 & 0.8 & 0.14 \\
\hline 9.24 & 380 & 5 & 500 & 0.83 & 0.12 \\
\hline 8.64 & 380 & 5 & 500 & 0.83 & 0.11 \\
\hline
\end{tabular}

\section{R134a with SUS150 ester-b oil}

\begin{tabular}{|c|c|c|c|c|c|}
\hline 0.63 & 132 & 1.1 & 500 & 0.84 & 0.1 \\
\hline 0.63 & 132 & 1.1 & 500 & 0.83 & 0.1 \\
\hline 1.94 & 202 & 1.1 & 500 & 0.84 & 0.13 \\
\hline 2.07 & 204 & 1.1 & 500 & 0.82 & 0.11 \\
\hline 5.15 & 300 & 1.1 & 500 & 0.82 & 0.13 \\
\hline 5.32 & 301 & 1.1 & 500 & 0.83 & 0.14 \\
\hline 7.96 & 380 & 1.1 & 500 & 0.88 & 0.11 \\
\hline 7.94 & 382 & 1.1 & 500 & 0.87 & 0.1 \\
\hline 0.58 & 121 & 2.4 & 500 & 0.86 & 0.14 \\
\hline 0.67 & 123 & 2.4 & 500 & 0.84 & 0.14 \\
\hline 1.97 & 208 & 2.4 & 500 & 0.83 & 0.08 \\
\hline 1.94 & 206 & 2.4 & 500 & 0.84 & 0.09 \\
\hline 5.02 & 310 & 2.4 & 500 & 0.83 & 0.1 \\
\hline 4.5 & 308 & 2.4 & 500 & 0.83 & 0.11 \\
\hline 7.21 & 371 & 2.4 & 500 & 0.82 & 0.12 \\
\hline 7.18 & 371 & 2.4 & 500 & 0.83 & 0.13 \\
\hline 0.76 & 127 & 5 & 500 & 0.81 & 0.1 \\
\hline 0.77 & 128 & 5 & 500 & 0.81 & 0.1 \\
\hline 1.91 & 202 & 5 & 500 & 0.87 & 0.08 \\
\hline 1.91 & 203 & 5 & 500 & 0.86 & 0.08 \\
\hline 5 & 299 & 5 & 500 & 0.83 & 0.11 \\
\hline 4.88 & 300 & 5 & 500 & 0.83 & 0.11 \\
\hline 8.25 & 367 & 5 & 500 & 0.82 & 0.15 \\
\hline 7.85 & 367 & 5 & 500 & 0.83 & 0.13 \\
\hline
\end{tabular}

\section{R22 with SUS150 mineral oil}

$\begin{array}{lllllll}0.9 & 127 & 1.3 & 800 & 0.94 & 0.11 & 1540 \\ 0.8 & 127 & 1.3 & 800 & 0.87 & 0.11 & 1530 \\ 0.7 & 128 & 1.3 & 800 & 0.92 & 0.1 & 1550 \\ 1 & 131 & 1.3 & 800 & 0.89 & 0.1 & 1590 \\ 1.7 & 198 & 1.3 & 800 & 0.9 & 0.14 & 1590 \\ 1.6 & 202 & 1.3 & 800 & 0.88 & 0.13 & 1580 \\ 2.9 & 300 & 1.3 & 800 & 0.85 & 0.11 & 1600 \\ 2.8 & 303 & 1.3 & 800 & 0.82 & 0.09 & 1590 \\ 2.8 & 303 & 1.3 & 800 & 0.86 & 0.09 & 1590 \\ 5.8 & 400 & 1.3 & 800 & 0.95 & 0.18 & 1610 \\ 6 & 401 & 1.3 & 800 & 0.91 & 0.17 & 1580 \\ 6 & 402 & 1.3 & 800 & 0.95 & 0.19 & 1620 \\ 5.8 & 409 & 1.3 & 800 & 0.87 & 0.14 & 1490 \\ 6.6 & 409 & 1.3 & 800 & 0.87 & 0.15 & 1490 \\ 0.7 & 122 & 2.4 & 800 & 0.83 & 0.17 & 1530 \\ 0.6 & 122 & 2.4 & 800 & 0.83 & 0.17 & 1520 \\ 0.6 & 122 & 2.4 & 800 & 0.83 & 0.17 & 1530 \\ 1.3 & 191 & 2.4 & 800 & 0.84 & 0.12 & 1540 \\ 1.2 & 192 & 2.4 & 800 & 0.83 & 0.13 & 1540 \\ 1.1 & 192 & 2.4 & 800 & 0.83 & 0.12 & 1550 \\ 2.4 & 292 & 2.4 & 800 & 0.84 & 0.1 & 1550\end{array}$

$0.0008728 \quad 0.0199$ $\begin{array}{lll}0.0008728 & 0.0199 & 1701\end{array}$ $\begin{array}{lll}0.0008704 & 0.02032 & 1718 \\ 0.0008706 & 0.02028 & 1717\end{array}$ $\begin{array}{lll}0.000868 & 0.02076 & 1736\end{array}$ $\begin{array}{lll}0.000869 & 0.02058 & 1729 \\ 0.0008693 & 0.02051 & 1726\end{array}$ $\begin{array}{lll}0.0008693 & 0.02051 & 1726 \\ 0.000871 & 0.02021 & 171\end{array}$ $\begin{array}{lll}0.000871 & 0.02021 \\ 0.0008713 & 0.02015\end{array}$ $0.0008706 \quad 0.02028$ $0.0008728 \quad 0.0199$ $\begin{array}{ll}0.0008728 & 0.0199\end{array}$ $\begin{array}{ll}0.0008703 & 0.02034 \\ 0.0008706 & 0.02028\end{array}$ $\begin{array}{ll}0.0008706 & 0.02028 \\ 0.0008672 & 0.02091\end{array}$ $\begin{array}{lll}0.0008672 & 0.02091 \\ 0.0008688 & 0.02098\end{array}$ 0.00086680 .02098 $\begin{array}{lll}0.0008694 & 0.02049\end{array}$ $0.0008696-0.02047$ $\begin{array}{ll}0.0008696 & 0.02047 \\ 0.0008724 & 0.01996\end{array}$ $\begin{array}{lll}0.0008718 & 0.02007\end{array}$ $\begin{array}{ll}0.000871 & 0.02021 \\ 0.000875 & 0.0213\end{array}$ $0.0008715 \quad 0.02013$ $\begin{array}{lll}0.0008717 & 0.02009 \\ 0.0008789 & 0.01888\end{array}$ $0.000878-0.01903$ $0.0008751-0.0195$ 0.00087440 .01961 $0.000873 \quad 0.01986$ $\begin{array}{lll}0.0008722 & 0.02\end{array}$ $\begin{array}{ll}0.0008703 & 0.02034\end{array}$ $\begin{array}{lll}0.0008706 & 0.02028\end{array}$ $\begin{array}{ll}0.0008716 & 0.02011\end{array}$ $\begin{array}{lll}0.0008711 & 0.02019 \\ 0.0008706 & 0.02028\end{array}$ $\begin{array}{lll}0.0008709 & 0.02023\end{array}$ $\begin{array}{lll}0.0008694 & 0.02049\end{array}$ $\begin{array}{lll}0.000869 & 0.020 \\ 0.000805 & 0.0203\end{array}$ $\begin{array}{ll}0.0008705 & 0.0203 \\ 0.0008708 & 0.02026\end{array}$ 1717
-0.0203

$\begin{array}{llll}128.6 & 40.2 & 954.5 & 0.0654148 \\ 128.6 & 40.1 & 954.5 & 0.0656882 \\ 128.2 & 39.4 & 955 & 0.0679459 \\ 128.2 & 39.5 & 954.9 & 0.0675669 \\ 127.8 & 38.6 & 955.4 & 0.0704673 \\ 127.9 & 38.9 & 955.3 & 0.06954 \\ 128 & 39.1 & 955.1 & 0.0688723 \\ 128.3 & 39.6 & 954.8 & 0.0672792 \\ 128.3 & 39.8 & 954.7 & 0.0666271 \\ 128.2 & 39.5 & 954.9 & 0.0675669 \\ 128 & 39 & 955.2 & 0.0691605 \\ 128.6 & 40.2 & 954.5 & 0.0654148 \\ 128.6 & 40.1 & 954.5 & 0.0656882 \\ 128.2 & 39.4 & 955 & 0.0679459 \\ 128.2 & 39.5 & 954.9 & 0.0675669 \\ 127.6 & 38.3 & 955.6 & 0.0715013 \\ 127.6 & 38.3 & 955.6 & 0.0715013 \\ 128 & 39.1 & 955.1 & 0.0688723 \\ 128 & 39.2 & 955.1 & 0.0685986 \\ 127.9 & 39 & 955.2 & 0.0691605 \\ 128 & 39.2 & 955.1 & 0.0685986 \\ 128.5 & 40.1 & 954.5 & 0.0656882 \\ 128.4 & 39.9 & 954.7 & 0.0663537 \\ 128.3 & 39.7 & 954.8 & 0.0670058 \\ 128.4 & 39.8 & 954.7 & 0.0666271 \\ 128.4 & 39.9 & 954.7 & 0.0663537 \\ 129.6 & 41.9 & 953.5 & 0.0604593 \\ 129.5 & 41.7 & 953.6 & 0.0610176 \\ 129 & 40.9 & 954.1 & 0.0633573 \\ 128.9 & 40.7 & 954.2 & 0.0639169 \\ 128.6 & 40.2 & 954.5 & 0.0654148 \\ 128.5 & 40.1 & 954.5 & 0.0656882 \\ 128.2 & 39.4 & 955 & 0.0679459 \\ 128.2 & 39.5 & 954.9 & 0.0675669 \\ 128.4 & 39.8 & 954.7 & 0.0666271 \\ 128.3 & 39.7 & 954.8 & 0.0670058 \\ 128.2 & 39.5 & 954.9 & 0.0675669 \\ 128.3 & 39.6 & 954.8 & 0.0672792 \\ 128 & 39.1 & 955.1 & 0.0688723 \\ 127.9 & 39 & 955.2 & 0.0691605 \\ 128.2 & 39.5 & 954.9 & 0.0675669 \\ 128.2 & 39.6 & 95.8 & 0.0672792\end{array}$

$\begin{array}{lll}0.0008778 & 0.01905 & 1665\end{array}$ $\begin{array}{lll}0.000875 & 0.01952 & 1685 \\ 0.0008722 & 0.02 & 1705\end{array}$ $\begin{array}{lll}0.0008729 & 0.01988 & 1700\end{array}$ $\begin{array}{lll}0.0008718 & 0.02007 & 1708 \\ 0.0008716 & 0.02011 & 1710 \\ 0.008747 & 0.057 & 1688\end{array}$ $\begin{array}{lll}0.0008747 & 0.01957 & 1688\end{array}$ $\begin{array}{lll}0.00008747 & 0.01957 & 1688\end{array}$ $\begin{array}{lll}0.0008761 & 0.01934 & 1678\end{array}$ $\begin{array}{lll}0.0008752 & 0.01948 & 1683\end{array}$ $\begin{array}{lll}0.0008735 & 0.01978 & 1696\end{array}$ $\begin{array}{lll}0.0008728 & 0.0199 & 1701\end{array}$ $\begin{array}{lll}0.0008752 & 0.01948 & 1683\end{array}$ $\begin{array}{lll}0.0008748 & 0.01955 & 1687 \\ 0.0008719 & 0.02004 & 1707\end{array}$ $\begin{array}{lll}0.0008712 & 0.02017\end{array}$ $\begin{array}{lll}0.0008705 & 0.0203\end{array}$ $\begin{array}{lll}0.0008709 & 0.02023 & 1715\end{array}$ $\begin{array}{lll}0.0008768 & 0.01922 & 1673\end{array}$ $\begin{array}{lll}0.0008765 & 0.01926 & 1674 \\ 0.0008721 & 0.02002 & 1706\end{array}$ $\begin{array}{lll}0.0008721 & 0.02002 & 1706 \\ 0.0008728 & 0.0199 & 170\end{array}$ $\begin{array}{lll}0.0008728 & 0.0199 & 1701 \\ 0.0008689 & 0.0206 & 1730\end{array}$ $\begin{array}{lll}0.0008689 & 0.0206 & 1730 \\ 0.0008709 & 0.02023 & 1715\end{array}$

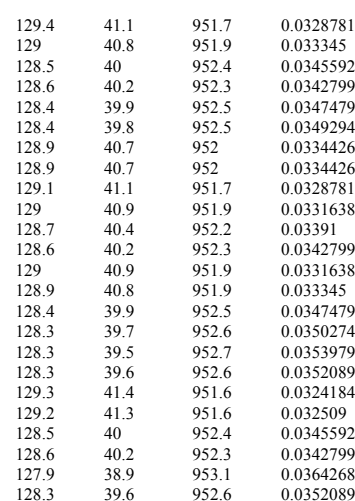

$\begin{array}{lll}0.000887 & 0.01479 & 1460\end{array}$ $\begin{array}{llll}137.9 & 40.2 & 952.3 & 0.0342799 \\ 137.8 & 39.9 & 952.5 & 0.0347479 \\ 138.1 & 40.4 & 952.2 & 0.03391 \\ 138.8 & 41.5 & 951.5 & 0.0322305 \\ 138.8 & 41.5 & 951.5 & 0.0322305 \\ 138.6 & 41.2 & 951.7 & 0.032697 \\ 138.9 & 41.7 & 951.4 & 0.0319522 \\ 138.8 & 41.5 & 951.5 & 0.0322305 \\ 138.8 & 41.5 & 951.5 & 0.0322305 \\ 139.1 & 42 & 951.2 & 0.0314864 \\ 138.6 & 41.2 & 951.7 & 0.032697 \\ 139.3 & 42.2 & 951.1 & 0.0312084 \\ 137.1 & 38.8 & 953.1 & 0.0366085 \\ 137.1 & 38.8 & 953.1 & 0.0366085 \\ 137.8 & 39.9 & 952.5 & 0.0347479 \\ 137.6 & 39.6 & 952.6 & 0.0352089 \\ 137.8 & 39.9 & 952.5 & 0.0347479 \\ 137.9 & 40.2 & 952.3 & 0.0342799 \\ 137.9 & 40.2 & 952.3 & 0.0342799 \\ 138.1 & 40.4 & 952.2 & 0.03391 \\ 138.1 & 40.4 & 952.2 & 0.03391\end{array}$ 


\begin{tabular}{|c|c|c|c|c|c|c|c|c|c|c|c|c|c|c|c|}
\hline 2.5 & 297 & 2.4 & 800 & 0.87 & 0.11 & 1570 & 86.47 & 162.9 & 0.0008898 & 0.01448 & 1447 & 138.4 & 40.9 & 951.9 & 0.0331638 \\
\hline 2.5 & 303 & 2.4 & 800 & 0.83 & 0.1 & 1570 & 86.47 & 162.9 & 0.0008898 & 0.01448 & 1447 & 138.4 & 40.9 & 951.9 & 0.0331638 \\
\hline 4.8 & 400 & 2.4 & 800 & 0.87 & 0.13 & 1550 & 86.47 & 163.5 & 0.0008879 & 0.01468 & 1456 & 138.1 & 40.4 & 952.2 & 0.03391 \\
\hline 4.7 & 400 & 2.4 & 800 & 0.87 & 0.13 & 1550 & 86.47 & 163.5 & 0.0008879 & 0.01468 & 1456 & 138.1 & 40.4 & 952.2 & 0.03391 \\
\hline 4.7 & 407 & 2.4 & 800 & 0.86 & 0.12 & 1550 & 86.47 & 163.5 & 0.0008879 & 0.01468 & 1456 & 138.1 & 40.4 & 952.2 & 0.03391 \\
\hline 0.8 & 128 & 4.9 & 800 & 0.93 & 0.1 & 1530 & 86.47 & 164.2 & 0.000886 & 0.01489 & 1464 & 137.8 & 39.9 & 952.5 & 0.0347479 \\
\hline 0.6 & 131 & 4.9 & 800 & 0.8 & 0.11 & 1550 & 86.47 & 163.5 & 0.0008879 & 0.01468 & 1456 & 138.1 & 40.4 & 952.2 & 0.03391 \\
\hline 0.9 & 131 & 4.9 & 800 & 0.8 & 0.14 & 1580 & 86.47 & 162.5 & 0.0008908 & 0.01438 & 1442 & 138.6 & 41.2 & 951.7 & 0.032697 \\
\hline 1.1 & 194 & 4.9 & 800 & 0.8 & 0.17 & 1630 & 86.47 & 161 & 0.0008956 & 0.01389 & 1420 & 139.4 & 42.5 & 950.9 & 0.0307432 \\
\hline 1.2 & 196 & 4.9 & 800 & 0.86 & 0.19 & 1680 & 86.47 & 159.5 & 0.0009003 & 0.01343 & 1399 & 140.3 & 43.8 & 950.1 & 0.0289763 \\
\hline 1.1 & 201 & 4.9 & 800 & 0.83 & 0.2 & 1690 & 86.47 & 159.1 & 0.0009013 & 0.01334 & 1395 & 140.4 & 44 & 950 & 0.0286995 \\
\hline 2.8 & 294 & 4.9 & 800 & 0.93 & 0.24 & 1550 & 86.47 & 163.5 & 0.0008879 & 0.01468 & 1456 & 138.1 & 40.4 & 952.2 & 0.03391 \\
\hline 2.4 & 300 & 4.9 & 800 & 0.9 & 0.22 & 1540 & 86.47 & 163.8 & 0.000887 & 0.01479 & 1460 & 137.9 & 40.2 & 952.3 & 0.0342799 \\
\hline 2.8 & 302 & 4.9 & 800 & 0.89 & 0.21 & 1550 & 86.47 & 163.5 & 0.0008879 & 0.01468 & 1456 & 138.1 & 40.4 & 952.2 & 0.03391 \\
\hline 4.2 & 382 & 4.9 & 800 & 0.86 & 0.18 & 1570 & 86.47 & 162.9 & 0.0008898 & 0.01448 & 1447 & 138.4 & 40.9 & 951.9 & 0.0331638 \\
\hline 4.7 & 291 & 4.9 & 800 & 0.87 & 0.21 & 1610 & 86.47 & 161.6 & 0.0008936 & 0.01408 & 1429 & 139.1 & 42 & 951.2 & 0.0314864 \\
\hline 4.7 & 393 & 4.9 & 800 & 0.86 & 0.2 & 1600 & 86.47 & 161.9 & 0.0008927 & 0.01418 & 1433 & 138.9 & 41.7 & 951.4 & 0.0319522 \\
\hline 4.6 & 394 & 4.9 & 800 & 0.86 & 0.2 & 1590 & 86.47 & 162.3 & 0.0008917 & 0.01428 & 1438 & 138.8 & 41.5 & 951.5 & 0.0322305 \\
\hline
\end{tabular}

\section{R22 with SUS300 mineral oil}

$\begin{array}{lllllll}1 & 127 & 0.6 & 800 & 0.9 & 0.14 & 1650 \\ 0.8 & 131 & 0.6 & 800 & 0.8 & 0.05 & 1620 \\ 1.1 & 132 & 0.6 & 800 & 0.92 & 0.27 & 1550 \\ 2.8 & 201 & 0.6 & 800 & 0.87 & 0.16 & 1550 \\ 2.3 & 201 & 0.6 & 800 & 0.87 & 0.16 & 1550 \\ 2.3 & 201 & 0.6 & 800 & 0.87 & 0.17 & 1550 \\ 4.9 & 294 & 0.6 & 800 & 0.86 & 0.17 & 1560 \\ 4.4 & 295 & 0.6 & 800 & 0.85 & 0.15 & 1560 \\ 4.8 & 297 & 0.6 & 800 & 0.86 & 0.18 & 1550 \\ 7.8 & 396 & 0.6 & 800 & 0.85 & 0.15 & 1590 \\ 7.7 & 397 & 0.6 & 800 & 0.85 & 0.15 & 1590 \\ 7.8 & 399 & 0.6 & 800 & 0.84 & 0.14 & 1590 \\ 0.8 & 126 & 1.3 & 800 & 0.84 & 0.1 & 1510 \\ 0.8 & 127 & 1.3 & 800 & 0.84 & 0.13 & 1500 \\ 0.8 & 127 & 1.3 & 800 & 0.84 & 0.13 & 1500 \\ 1.9 & 202 & 1.3 & 800 & 0.84 & 0.1 & 1550 \\ 1.8 & 202 & 1.3 & 800 & 0.84 & 0.09 & 1550 \\ 1.7 & 205 & 1.3 & 800 & 0.82 & 0.11 & 1550 \\ 4.6 & 296 & 1.3 & 800 & 0.89 & 0.13 & 1540 \\ 4.2 & 298 & 1.3 & 800 & 0.83 & 0.12 & 1520 \\ 4.4 & 299 & 1.3 & 800 & 0.84 & 0.11 & 1520 \\ 7.1 & 397 & 1.3 & 800 & 0.86 & 0.13 & 1560 \\ 6.6 & 399 & 1.3 & 800 & 0.86 & 0.12 & 1570 \\ 7 & 402 & 1.3 & 800 & 0.84 & 0.11 & 1570 \\ 1.2 & 124 & 2.6 & 800 & 0.85 & 0.14 & 1520 \\ 1 & 125 & 2.6 & 800 & 0.84 & 0.15 & 1520 \\ 1.2 & 127 & 2.6 & 800 & 0.82 & 0.1 & 1510 \\ 2.2 & 200 & 2.6 & 800 & 0.86 & 0.16 & 1500 \\ 1.9 & 202 & 2.6 & 800 & 0.84 & 0.16 & 1500 \\ 2.4 & 204 & 2.6 & 800 & 0.84 & 0.14 & 1500 \\ 4.2 & 295 & 2.6 & 800 & 0.85 & 0.13 & 1510 \\ 4.6 & 300 & 2.6 & 800 & 0.83 & 0.12 & 1510 \\ 4.3 & 301 & 2.6 & 800 & 0.86 & 0.15 & 1520 \\ 7.3 & 392 & 2.6 & 800 & 0.84 & 0.11 & 1500 \\ 7.5 & 396 & 2.6 & 800 & 0.86 & 0.12 & 1510 \\ 7.4 & 398 & 2.6 & 800 & 0.86 & 0.13 & 1500 \\ 0.7 & 127 & 5 & 800 & 0.85 & 0.14 & 1570 \\ 0.8 & 130 & 5 & 800 & 0.83 & 0.1 & 1550 \\ 1 & 130 & 5 & 800 & 0.82 & 0.13 & 1560 \\ 2 & 201 & 5 & 800 & 0.85 & 0.09 & 1570 \\ 2 & 201 & 5 & 800 & 0.86 & 0.08 & 1570 \\ 1.8 & 203 & 5 & 800 & 0.8 & 0.06 & 1560 \\ 4.1 & 293 & 5 & 800 & 0.88 & 0.13 & 1560 \\ 3.9 & 296 & 5 & 800 & 0.87 & 0.12 & 1570 \\ 4.5 & 301 & 5 & 800 & 0.82 & 0.12 & 1550 \\ 7.2 & 398 & 5 & 800 & 0.88 & 0.12 & 1580 \\ 8 & 403 & 5 & 800 & 0.87 & 0.13 & 1570 \\ 7.6 & 403 & 5 & 800 & 0.86 & 0.12 & 1580 \\ & & & & & & \\ & & & & \end{array}$

\begin{tabular}{|c|c|c|c|c|c|c|c|c|}
\hline 86.47 & 160.4 & 0.0008975 & 0.0137 & 1412 & 139.8 & 43 & 952.1 & 0.0465938 \\
\hline 86.47 & 161.3 & 0.0008946 & 0.01399 & 1425 & 139.3 & 42.2 & 952.6 & 0.0483669 \\
\hline 86.47 & 163.5 & 0.0008879 & 0.01468 & 1456 & 138.1 & 40.4 & 953.7 & 0.0526626 \\
\hline 86.47 & 163.5 & 0.0008879 & 0.01468 & 1456 & 138.1 & 40.4 & 953.7 & 0.0526626 \\
\hline 86.47 & 163.5 & 0.0008879 & 0.01468 & 1456 & 138.1 & 40.4 & 953.7 & 0.0526626 \\
\hline 86.47 & 163.5 & 0.0008879 & 0.01468 & 1456 & 138.1 & 40.4 & 953.7 & 0.0526626 \\
\hline 86.47 & 163.2 & 0.0008889 & 0.01458 & 1451 & 138.3 & 40.7 & 953.5 & 0.0519132 \\
\hline 86.47 & 163.2 & 0.0008889 & 0.01458 & 1451 & 138.3 & 40.7 & 953.5 & 0.0519132 \\
\hline 86.47 & 163.5 & 0.0008879 & 0.01468 & 1456 & 138.1 & 40.4 & 953.7 & 0.0526626 \\
\hline 86.47 & 162.3 & 0.0008917 & 0.01428 & 1438 & 138.8 & 41.5 & 953 & 0.0500423 \\
\hline 86.47 & 162.3 & 0.0008917 & 0.01428 & 1438 & 138.8 & 41.5 & 953 & 0.0500423 \\
\hline 86.47 & 162.3 & 0.0008917 & 0.01428 & 1438 & 138.8 & 41.5 & 953 & 0.0500423 \\
\hline 86.47 & 164.8 & 0.0008841 & 0.01511 & 1474 & 137.4 & 39.4 & 954.3 & 0.0551877 \\
\hline 86.47 & 165 & 0.0008832 & 0.01522 & 1478 & 137.3 & 39.1 & 954.4 & 0.055928 \\
\hline 86.47 & 165 & 0.0008832 & 0.01522 & 1478 & 137.3 & 39.1 & 954.4 & 0.055928 \\
\hline 86.47 & 163.5 & 0.0008879 & 0.01468 & 1456 & 138.1 & 40.4 & 953.7 & 0.05266 \\
\hline 86.47 & 163.5 & 0.0008879 & 0.01468 & 1456 & 138.1 & 40.4 & 953.7 & 0.0526626 \\
\hline 86.47 & 163.5 & 0.0008879 & 0.01468 & 1456 & 138.1 & 40.4 & 953.7 & 0.0526626 \\
\hline 86.47 & 163.8 & 0.000887 & 0.01479 & 1460 & 137.9 & 40.2 & 953.8 & 0.05312 \\
\hline 86.47 & 164.4 & 0.0008851 & 0.015 & 1469 & 137.6 & 39.6 & 954.1 & 0.05461 \\
\hline 86.47 & 164.4 & 0.0008851 & 0.015 & 1469 & 137.6 & 39.6 & 954.1 & 0.05461 \\
\hline 86.47 & 163.2 & 0.0008889 & 0.01458 & 1451 & 138.3 & 40.7 & 953.5 & 0.05191 \\
\hline 86.47 & 162.9 & 0.0008898 & 0.01448 & 1447 & 138.4 & 40.9 & 953.4 & 0.05144 \\
\hline 86.47 & 162.9 & 0.0008898 & 0.01448 & 1447 & 138.4 & 40.9 & 953.4 & 0.05144 \\
\hline 86.47 & 164.4 & 0.0008851 & 0.015 & 1469 & 137.6 & 39.6 & 954.1 & 0.05461 \\
\hline 86.47 & 164.4 & 0.0008851 & 0.015 & 1469 & 137.6 & 39.6 & 954.1 & 0.05461 \\
\hline 86.47 & 164.8 & 0.0008841 & 0.01511 & 1474 & 137.4 & 39.4 & 954.3 & 0.0551877 \\
\hline 86.47 & 165 & 0.0008832 & 0.01522 & 1478 & 137.3 & 39.1 & 954.4 & 0.055928 \\
\hline 86.47 & 165 & 0.0008832 & 0.01522 & 1478 & 137.3 & 39.1 & 954.4 & 0.055928 \\
\hline 86.47 & 165 & 0.0008832 & 0.01522 & 1478 & 137.3 & 39.1 & 954.4 & 0.055928 \\
\hline 86.47 & 164.8 & 0.0008841 & 0.01511 & 1474 & 137.4 & 39.4 & 954.3 & 0.0551877 \\
\hline 86.47 & 164.8 & 0.0008841 & 0.01511 & 1474 & 137.4 & 39.4 & 954.3 & 0.05518 \\
\hline 86.47 & 164.4 & 0.0008851 & 0.015 & 1469 & 137.6 & 39.6 & 954.1 & 0.05461 \\
\hline 86.47 & 165 & 0.0008832 & 0.01522 & 1478 & 137.3 & 39.1 & 954.4 & 0.05592 \\
\hline 86.47 & 164.8 & 0.0008841 & 0.01511 & 1474 & 137.4 & 39.4 & 954.3 & 0.0551877 \\
\hline 86.47 & 165 & 0.0008832 & 0.01522 & 1478 & 137.3 & 39.1 & 954.4 & 0.05592 \\
\hline 86.47 & 162.9 & 0.0008898 & 0.01448 & 1447 & 138.4 & 40.9 & 953.4 & 0.05144 \\
\hline 86.47 & 163.5 & 0.0008879 & 0.01468 & 1456 & 138.1 & 40.4 & 953.7 & 0.05266 \\
\hline 86.47 & 163.2 & 0.0008889 & 0.01458 & 1451 & 138.3 & 40.7 & 953.5 & 0.0519132 \\
\hline 86.47 & 162.9 & 0.0008898 & 0.01448 & 1447 & 138.4 & 40.9 & 953.4 & 0.05144 \\
\hline 86.47 & 162.9 & 0.0008898 & 0.01448 & 1447 & 138.4 & 40.9 & 953.4 & 0.0514 \\
\hline 86.47 & 163.2 & 0.0008889 & 0.01458 & 1451 & 138.3 & 40.7 & 953.5 & 0.05191 \\
\hline 86.47 & 163.2 & 0.0008889 & 0.01458 & 1451 & 138.3 & 40.7 & 953.5 & 0.05191 \\
\hline 86.47 & 162.9 & 0.0008898 & 0.01448 & 1447 & 138.4 & 40.9 & 953.4 & 0.0514 \\
\hline 86.47 & 163.5 & 0.0008879 & 0.01468 & 1456 & 138.1 & 40.4 & 953.7 & 0.05266 \\
\hline 86.47 & 162.5 & 0.0008908 & 0.01438 & 1442 & 138.6 & 41.2 & 953.2 & 0.05069 \\
\hline 86.47 & 162.9 & 0.0008898 & 0.01448 & 1447 & 138.4 & 40.9 & 953.4 & 0051 \\
\hline 8647 & 162.5 & 0.0008908 & & 1442 & & & 953.2 & 0.050 \\
\hline
\end{tabular}

Smooth $3 / 8$ inch, evaporation

R12 with SUS150 naphthenic oil

$\begin{array}{lllllll}5.24 & 130 & 1.3 & 800 & 0.08 & 0.83 & 322 \\ 6 & 131 & 1.3 & 800 & 0.08 & 0.84 & 321 \\ 12.96 & 196 & 1.3 & 800 & 0.13 & 0.85 & 305 \\ 13.1 & 199 & 1.3 & 800 & 0.12 & 0.84 & 308 \\ 27.18 & 301 & 1.3 & 800 & 0.13 & 0.86 & 324 \\ 26.81 & 302 & 1.3 & 800 & 0.13 & 0.85 & 323 \\ 35.21 & 361 & 1.3 & 800 & 0.12 & 0.84 & 329 \\ 35.3 & 363 & 1.3 & 800 & 0.12 & 0.84 & 330 \\ 35.93 & 361 & 1.3 & 800 & 0.12 & 0.85 & 326 \\ 5.73 & 126 & 2.4 & 800 & 0.13 & 0.83 & 321 \\ 5.76 & 126 & 2.4 & 800 & 0.13 & 0.81 & 321 \\ 14.84 & 201 & 2.4 & 800 & 0.09 & 0.83 & 306 \\ 12.7 & 200 & 2.4 & 800 & 0.09 & 0.79 & 323 \\ 12.65 & 198 & 2.4 & 800 & 0.08 & 0.77 & 329 \\ 24.89 & 293 & 2.4 & 800 & 0.13 & 0.79 & 337 \\ 27.48 & 307 & 2.4 & 800 & 0.13 & 0.8 & 329 \\ 39.58 & 380 & 2.4 & 800 & 0.11 & 0.79 & 339 \\ 4.26 & 378 & 2.4 & 800 & 0.12 & 0.8 & 317 \\ 7.35 & 136 & 4.9 & 800 & 0.08 & 0.8 & 326 \\ 7.58 & 135 & 4.9 & 800 & 0.09 & 0.82 & 325 \\ 14.42 & 201 & 4.9 & 800 & 0.09 & 0.78 & 334 \\ 14.87 & 197 & 4.9 & 800 & 0.1 & 0.8 & 330 \\ 15.54 & 210 & 4.9 & 800 & 0.08 & 0.76 & 333 \\ 31.55 & 287 & 4.9 & 800 & 0.15 & 0.85 & 334 \\ 31.63 & 282 & 4.9 & 800 & 0.15 & 0.87 & 330 \\ 39.45 & 373 & 4.9 & 800 & 0.13 & 0.78 & 341 \\ 39.72 & 358 & 4.9 & 800 & 0.14 & 0.81 & 335 \\ 40.22 & 361 & 4.9 & 800 & 0.13 & 0.81 & 337\end{array}$
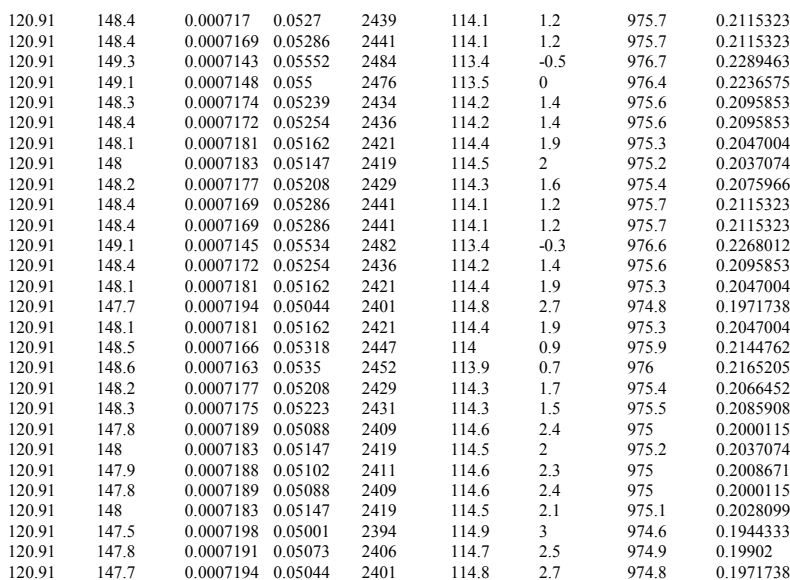

R12 with SUS300 naphthenic oil
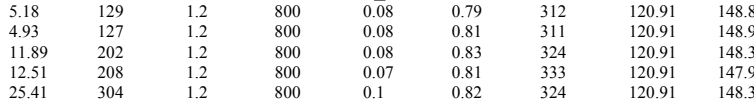

$\begin{array}{lll}0.0007155 & 0.05432 & 2465 \\ 0.007153 & 0.05449 & 2468\end{array}$ $\begin{array}{lll}0.0007153 & 0.05449 & 2468 \\ 0.0007174 & 0.05239 & 2434\end{array}$ $\begin{array}{lll}0.0007174 & 0.05239 & 2434 \\ 0.0007188 & 0.05102 & 2411 \\ 0.0007174 & 0.05239 & 2434\end{array}$

$\begin{array}{llll}113.7 & 0.3 & 977.7 & 0.3421157 \\ 113.6 & 0.2 & 977.8 & 0.3437154 \\ 114.2 & 1.4 & 977.1 & 0.3250837 \\ 114.6 & 2.3 & 976.5 & 0.3116209 \\ 114.2 & 1.5 & 977 & 0.3234899\end{array}$ 
$\begin{array}{lllllll}25.05 & 304 & 1.2 & 800 & 0.1 & 0.82 & 325 \\ 34.91 & 371 & 1.2 & 800 & 0.09 & 0.81 & 317 \\ 34.31 & 373 & 1.2 & 800 & 0.09 & 0.8 & 319 \\ 5.86 & 129 & 2.4 & 800 & 0.1 & 0.81 & 324 \\ 5.96 & 128 & 2.4 & 800 & 0.1 & 0.81 & 326 \\ 14.15 & 205 & 2.4 & 800 & 0.09 & 0.83 & 327 \\ 15.38 & 206 & 2.4 & 800 & 0.09 & 0.83 & 327 \\ 27.34 & 303 & 2.4 & 800 & 0.11 & 0.8 & 316 \\ 28.39 & 303 & 2.4 & 800 & 0.11 & 0.8 & 313 \\ 41.11 & 375 & 2.4 & 800 & 0.1 & 0.8 & 305 \\ 42.22 & 371 & 2.4 & 800 & 0.11 & 0.83 & 303 \\ 6.45 & 127 & 4.8 & 800 & 0.12 & 0.82 & 330 \\ 18.26 & 195 & 4.8 & 800 & 0.12 & 0.79 & 304 \\ 16.88 & 197 & 4.8 & 800 & 0.11 & 0.78 & 316 \\ 37.31 & 299 & 4.8 & 800 & 0.14 & 0.85 & 312 \\ 36.46 & 297 & 4.8 & 800 & 0.14 & 0.85 & 314 \\ 36.71 & 295 & 4.8 & 800 & 0.14 & 0.86 & 314 \\ 44.7 & 359 & 4.8 & 800 & 0.13 & 0.82 & 327 \\ 45.35 & 360 & 4.8 & 800 & 0.13 & 0.82 & 326 \\ \mathbf{R} & & & & \end{array}$

R134a with SUS169 ester-m oil

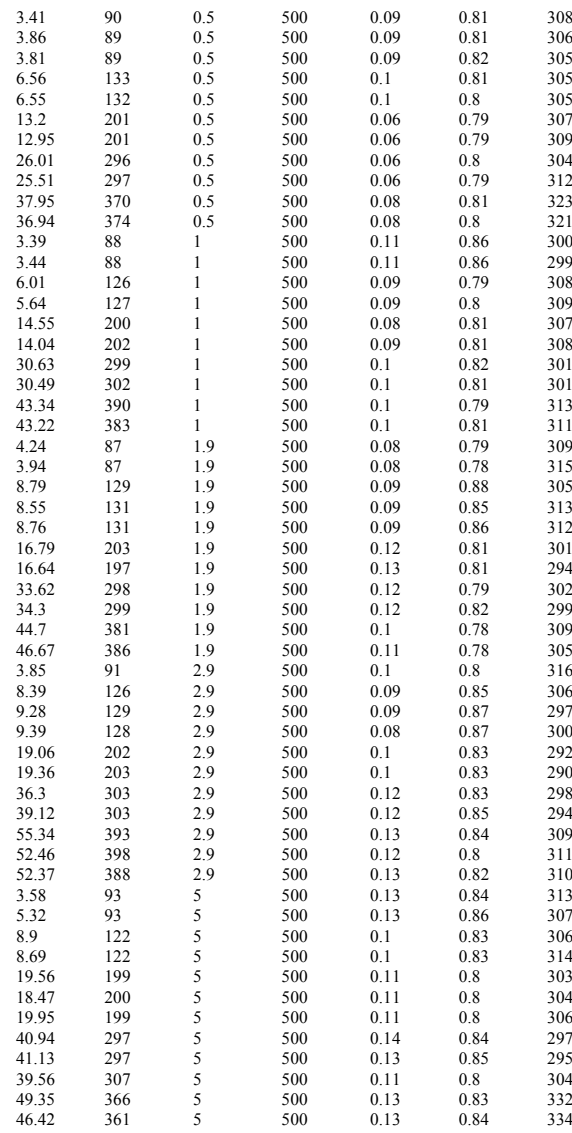

R134a with SUS369 ester-m oil

$\begin{array}{lllllllll}120.91 & 148.3 & 0.0007175 & 0.05223 & 2431 & 114.3 & 1.6 & 976.9 & 0.3219922 \\ 120.91 & 148.6 & 0.0007163 & 0.0555 & 2452 & 113.9 & 0.8 & 977.4 & 0.3342633 \\ 120.91 & 148.5 & 0.0007166 & 0.05318 & 2447 & 114 & 0.9 & 977.4 & 0.3327348 \\ 120.91 & 148.3 & 0.0007174 & 0.05239 & 2434 & 114.2 & 1.4 & 977.1 & 0.3250837 \\ 120.91 & 148.2 & 0.0007177 & 0.05208 & 2429 & 114.3 & 1.6 & 976.9 & 0.3219922 \\ 120.91 & 148.1 & 0.0007178 & 0.05192 & 2426 & 114.3 & 1.8 & 976.8 & 0.3189684 \\ 120.91 & 148.1 & 0.0007178 & 0.05192 & 2426 & 114.3 & 1.8 & 976.8 & 0.3189684 \\ 120.91 & 148.7 & 0.0007161 & 0.05366 & 2455 & 113.9 & 0.7 & 977.5 & 0.3358605 \\ 120.91 & 148.9 & 0.0007156 & 0.05516 & 2463 & 113.7 & 0.4 & 977.7 & 0.34505862 \\ 120.91 & 149.3 & 0.0007143 & 0.05552 & 2484 & 113.4 & -0.4 & 978.1 & 0.3534931 \\ 120.91 & 149.3 & 0.000714 & 0.05587 & 2490 & 113.3 & -0.6 & 978.3 & 0.3568917 \\ 120.91 & 148 & 0.0007183 & 0.05147 & 2419 & 114.5 & 2 & 976.7 & 0.3160413 \\ 120.91 & 149.3 & 0.0007142 & 0.05569 & 2487 & 113.3 & -0.5 & 978.2 & 0.3551921 \\ 120.91 & 148.7 & 0.0007161 & 0.05366 & 2455 & 113.9 & 0.7 & 977.5 & 0.3358605 \\ 120.91 & 148.8 & 0.0007155 & 0.05532 & 2465 & 113.7 & 0.3 & 977.7 & 0.3421157 \\ 120.91 & 148.8 & 0.0007158 & 0.05399 & 2460 & 113.8 & 0.5 & 977.6 & 0.3389874 \\ 120.91 & 148.8 & 0.0007158 & 0.05399 & 2460 & 113.8 & 0.4 & 977.7 & 0.3405862 \\ 120.91 & 148.1 & 0.0007178 & 0.05192 & 2426 & 114.3 & 1.7 & 976.9 & 0.3205607 \\ 120.91 & 148.2 & 0.0007177 & 0.05208 & 2429 & 114.3 & 1.6 & 976.9 & 0.3219922\end{array}$

$\begin{array}{lllll}102.03 & 197.6 & 0.0007758 & 0.06602 & 2826\end{array}$

$\begin{array}{llll}109.8 & 1.5 & 975.7 & 0.2235274\end{array}$ $\begin{array}{llll}109.8 & 1.5 & 975.7 & 0.2235274 \\ 109.7 & 1.3 & 975.8 & 0.2255728 \\ 109.7 & 1.2 & 975.9 & 0.2277143\end{array}$ $\begin{array}{llll}109.7 & 1.2 & 975.9 & 0.2266666 \\ 109.7 & 1.1 & 975.9 & 0.2277143\end{array}$ $\begin{array}{llll}109.7 & 1.2 & 975.9 & 0.2266666 \\ 109.8 & 1.3 & 975.8 & 0.2255728\end{array}$ $\begin{array}{llll}109.8 & 1.3 & 975.8 & 0.2255728 \\ 109.9 & 1.5 & 975.7 & 0.2235274\end{array}$ $\begin{array}{llll}109.7 & 1.1 & 975.9 & 0.2277143 \\ 110 & 1.7 & 975.6 & 0.2214828\end{array}$ $\begin{array}{llll}110 & 1.7 & 975.6 & 0.2214828 \\ 110.4 & 2.7 & 975 & 0.2113239\end{array}$ $\begin{array}{llll}110.4 & 2.7 & 975 & 0.2143239 \\ 110.3 & 2.5 & 975.1 & 0.213364 \\ 109.5 & 0.7 & 976.2 & 0.2320473\end{array}$ $\begin{array}{llll}109.5 & 0.7 & 976.2 & 0.2320473 \\ 109.4 & 0.6 & 976.2 & 0.2330956\end{array}$ $\begin{array}{llll}109.4 & 0.6 & 976.2 & 0.2330656 \\ 109.8 & 1.4 & 975.8 & 0.2246206 \\ 109.9 & 1.5 & 975.7 & 0.2235274\end{array}$ $\begin{array}{llll}109.9 & 1.5 & 975.7 & 0.2235274 \\ 109.8 & 1.3 & 975.8 & 0.2255728\end{array}$ $\begin{array}{llll}109.8 & 1.4 & 975.8 & 0.2246206 \\ 109.5 & 0.8 & 976.1 & 0.2309517\end{array}$ $\begin{array}{llll}109.5 & 0.8 & 976.1 & 0.2309517 \\ 109.5 & 0.8 & 976.1 & 0.2309517\end{array}$ 109.9 109.9
110.1 109.7 110
110 110
109.5
109.2 109.2

$\begin{array}{llll}109.7 & 1.2 & 977.9 & 0.4039363 \\ 109.6 & 0.9 & 978.1 & 0.4096502 \\ 110.1 & 2.1 & 977.3 & 0.3872993 \\ 109.5 & 0.8 & 978.1 & 0.4114679 \\ 109.3 & 0.2 & 978.5 & 0.4231983 \\ 109.1 & 0 & 978.6 & 0.4272112 \\ 109.7 & 1.3 & 977.8 & 0.402037 \\ 109.7 & 1.1 & 977.9 & 0.4057532 \\ 109.3 & 0.4 & 978.4 & 0.4192828 \\ 110.3 & 2.3 & 977.2 & 0.3836868 \\ 110.1 & 2.1 & 977.3 & 0.3872993 \\ 110 & 1.8 & 977.5 & 0.3927131 \\ 109.8 & 1.4 & 977.8 & 0.4002205 \\ 109.6 & 0.9 & 978.1 & 0.4096502 \\ 109.4 & 0.7 & 978.2 & 0.4134658 \\ 109.6 & 0.9 & 978.1 & 0.4096502 \\ 109.8 & 1.4 & 977.8 & 0.4002205 \\ 109.8 & 1.4 & 977.8 & 0.4002205 \\ 109.8 & 1.4 & 977.8 & 0.4002205 \\ 109.9 & 1.7 & 977.6 & 0.3946093 \\ 110 & 1.8 & 977.5 & 0.3927131 \\ 109.9 & 1.7 & 977.6 & 0.3946093 \\ 110 & 1.8 & 977.5 & 0.3927131 \\ 110 & 1.8 & 977.5 & 0.3927131 \\ 109.9 & 1.5 & 977.7 & 0.3983224 \\ 110.3 & 2.3 & 977.2 & 0.3836868 \\ 109.7 & 1.2 & 977.9 & 0.4039363 \\ 109.8 & 1.4 & 977.8 & 0.4002205 \\ 108.9 & -0.6 & 979 & 0.4393494 \\ 110 & 1.9 & 977.5 & 0.3909931 \\ 109.3 & 0.3 & 978.4 & 0.4211973\end{array}$



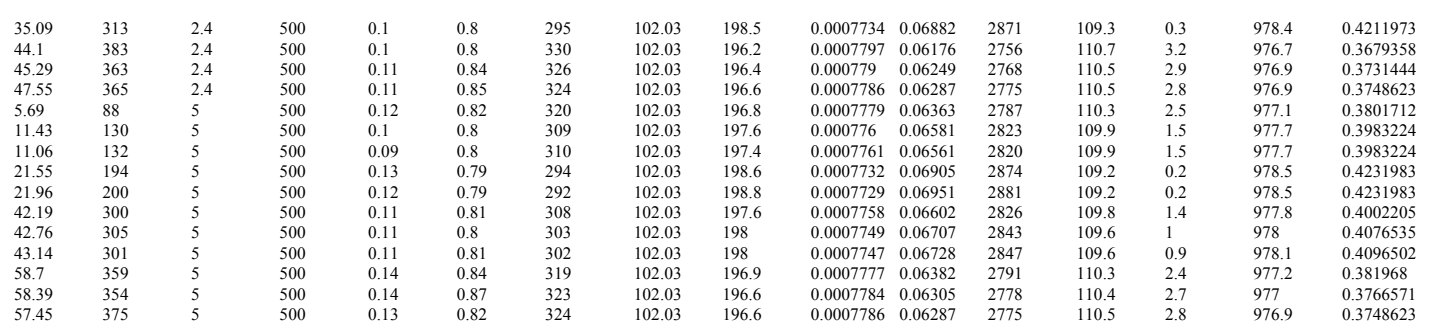

R134a with SUS150 ester-b oil
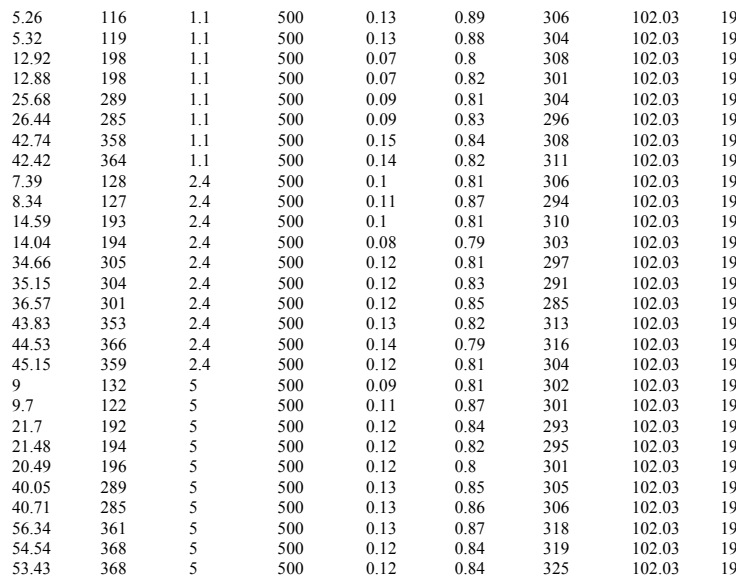

$\begin{array}{lll}0.0007754 & 0.06644 & 2833\end{array}$

$\begin{array}{llll}109.7 & 1.2 & 975.7 & 0.2115323 \\ 109.7 & 1.1 & 975.7 & 0.2124843 \\ 109.8 & 1.4 & 975.6 & 0.2095853 \\ 109.5 & 0.8 & 955.9 & 0.2554285 \\ 109.7 & 1.1 & 975.7 & 0.2124843 \\ 109.3 & 0.4 & 976.2 & 0.2195635 \\ 109.8 & 1.5 & 975.5 & 0.2085908 \\ 109.9 & 1.6 & 975.4 & 0.2075966 \\ 109.7 & 1.3 & 975.6 & 0.2105371 \\ 109.2 & 0.2 & 976.3 & 0.2216101 \\ 109.9 & 1.6 & 975.4 & 0.2075966 \\ 109.6 & 1 & 975.8 & 0.21348 \\ 109.4 & 0.4 & 976.2 & 0.2195635 \\ 109.1 & 0 & 976.4 & 0.2236575 \\ 108.9 & -0.7 & 976.8 & 0.2310923 \\ 110 & 1.9 & 975.3 & 0.2047004 \\ 110.1 & 2.2 & 975.1 & 0.2018591 \\ 109.7 & 1.1 & 975.7 & 0.2124843 \\ 109.6 & 0.9 & 975.9 & 0.2144762 \\ 109.5 & 0.8 & 975.9 & 0.2154285 \\ 109.2 & 0.2 & 976.3 & 0.2216101 \\ 109.3 & 0.3 & 976.2 & 0.2205164 \\ 109.5 & 0.8 & 975.9 & 0.2154285 \\ 109.7 & 1.1 & 975.7 & 0.2124843 \\ 109.7 & 1.3 & 975.6 & 0.2105371 \\ 110.2 & 2.3 & 975 & 0.2008671 \\ 110.3 & 2.4 & 975 & 0.2000115 \\ 110.5 & 2.9 & 974.7 & 0.1954232\end{array}$

\section{R22 with SUS150 mineral oil}

$\begin{array}{lllllll}3.7 & 125 & 1.3 & 800 & 0.13 & 0.81 & 490 \\ 3.3 & 125 & 1.3 & 800 & 0.11 & 0.74 & 520 \\ 3.7 & 127 & 1.3 & 800 & 0.11 & 0.73 & 510 \\ 9.9 & 205 & 1.3 & 800 & 0.12 & 0.78 & 490 \\ 9.4 & 206 & 1.3 & 800 & 0.12 & 0.77 & 500 \\ 9.7 & 208 & 1.3 & 800 & 0.12 & 0.78 & 500 \\ 19.4 & 283 & 1.3 & 800 & 0.16 & 0.75 & 480 \\ 19.5 & 287 & 1.3 & 800 & 0.16 & 0.74 & 480 \\ 30 & 388 & 1.3 & 800 & 0.15 & 0.69 & 500 \\ 30.8 & 393 & 1.3 & 800 & 0.15 & 0.69 & 490 \\ 30.8 & 397 & 1.3 & 800 & 0.15 & 0.64 & 480 \\ 3.7 & 117 & 2.4 & 800 & 0.18 & 0.79 & 480 \\ 4.6 & 118 & 2.4 & 800 & 0.17 & 0.81 & 480 \\ 4.1 & 119 & 2.4 & 800 & 0.18 & 0.75 & 470 \\ 8.7 & 186 & 2.4 & 800 & 0.14 & 0.76 & 480 \\ 8.6 & 193 & 2.4 & 800 & 0.16 & 0.72 & 490 \\ 9.3 & 194 & 2.4 & 800 & 0.16 & 0.72 & 490 \\ 9.8 & 198 & 2.4 & 800 & 0.16 & 0.75 & 490 \\ 10.5 & 200 & 2.4 & 800 & 0.15 & 0.73 & 490 \\ 20 & 292 & 2.4 & 800 & 0.15 & 0.78 & 530 \\ 20 & 293 & 2.4 & 800 & 0.15 & 0.74 & 520 \\ 24.5 & 294 & 2.4 & 800 & 0.14 & 0.81 & 520 \\ 21 & 298 & 2.4 & 800 & 0.13 & 0.78 & 520 \\ 19.8 & 300 & 2.4 & 800 & 0.13 & 0.75 & 520 \\ 32.5 & 388 & 2.4 & 800 & 0.12 & 0.81 & 560 \\ 30.2 & 398 & 2.4 & 800 & 0.11 & 0.75 & 560 \\ 4.2 & 119 & 4.9 & 800 & 0.23 & 0.78 & 550 \\ 4.3 & 122 & 4.9 & 800 & 0.21 & 0.78 & 560 \\ 4.4 & 130 & 4.9 & 800 & 0.19 & 0.76 & 550 \\ 14 & 197 & 4.9 & 800 & 0.16 & 0.85 & 520 \\ 14.4 & 198 & 4.9 & 800 & 0.16 & 0.88 & 520 \\ 11.9 & 200 & 4.9 & 800 & 0.15 & 0.81 & 540 \\ 21.4 & 281 & 4.9 & 800 & 0.15 & 0.85 & 520 \\ 20.8 & 288 & 4.9 & 800 & 0.15 & 0.81 & 530 \\ 20.4 & 298 & 4.9 & 800 & 0.15 & 0.74 & 550 \\ 35.9 & 388 & 4.9 & 800 & 0.2 & 0.84 & 580 \\ 34.2 & 389 & 4.9 & 800 & 0.21 & 0.79 & 550 \\ \mathbf{R} & & & & \end{array}$

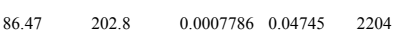

$\begin{array}{llll}116.4 & -0.5 & 976.7 & 0.2289463\end{array}$

\begin{tabular}{|c|c|c|c|c|c|c|c|c|}
\hline & & & & & & & & \\
\hline 86.47 & 201.4 & 0.0007823 & 0.04478 & 2166 & 117.3 & 1.3 & 975.6 & 0.2105371 \\
\hline 86.47 & 201.9 & 0.0007811 & 0.04564 & 2178 & 117 & 0.7 & 976 & 0.2165205 \\
\hline 86.47 & 202.8 & 0.0007786 & 0.04745 & 2204 & 116.4 & -0.5 & 976.7 & 0.2289463 \\
\hline 86.47 & 202.3 & 0.0007798 & 0.04653 & 2191 & 116.7 & 0.1 & 976.3 & 0.2226586 \\
\hline 86.47 & 202.3 & 0.0007798 & 0.04653 & 2191 & 116.7 & 0.1 & 976.3 & 0.2226586 \\
\hline 86.47 & 203.3 & 0.0007773 & 0.04841 & 2218 & 116.1 & -1.1 & 977 & 0.2343369 \\
\hline 86.47 & 203.3 & 0.0007773 & 0.04841 & 2218 & $\begin{array}{l}116.1 \\
116.1\end{array}$ & -1.1 & 977 & 0.2343369 \\
\hline 86.47 & 202.3 & 0.0007798 & 0.04653 & 2191 & 116.7 & 0.1 & 976.3 & 0.2226586 \\
\hline 86.47 & 202.8 & 0.0007786 & 0.04745 & 2204 & 116.4 & $\begin{array}{l}.1 .5 \\
-0.5\end{array}$ & 976.7 & 0.2289463 \\
\hline 86.47 & 203.3 & 0.0007773 & 0.04841 & 2218 & 116.1 & -1.1 & 977 & 0.2343369 \\
\hline 86.47 & 203.3 & 0.0007773 & 0.04841 & 2218 & 116.1 & -1.1 & 977 & 0.2343369 \\
\hline 86.47 & 203.3 & 0.0007773 & 0.04841 & 2218 & 116.1 & -1.1 & 977 & 0.2343369 \\
\hline 86.47 & 203.8 & 0.000776 & $\begin{array}{l}0.0404941 \\
0.0491\end{array}$ & 2231 & $\begin{array}{l}115.1 \\
115.8\end{array}$ & $\begin{array}{l}-1.11 \\
-1.7\end{array}$ & 977.3 & $\begin{array}{l}0.2397339 \\
0.239\end{array}$ \\
\hline 86.47 & 203.3 & 0.0007773 & 0.04841 & 2218 & 116.1 & -1.1 & 977 & 0.2343369 \\
\hline 86.47 & 202.8 & 0.0007786 & 0.04745 & 2204 & 116.4 & -0.5 & 976.7 & 0.2289463 \\
\hline 86.47 & 202.8 & 0.0007786 & 0.04745 & 2204 & 116.4 & -0.5 & 976.7 & 0.2289463 \\
\hline 86.47 & 202.8 & 0.0007786 & 0.04745 & 2204 & $\begin{array}{l}116.4 \\
116.4\end{array}$ & -0.5 & 976.7 & 0.2289463 \\
\hline 86.47 & 202.8 & 0.0007786 & 0.04745 & 2204 & 116.4 & -0.5 & 976.7 & 0.2289463 \\
\hline 86.47 & 200.9 & 0.0007836 & 0.04396 & 2153 & 117.5 & 1.9 & 975.3 & 0.2047004 \\
\hline 86.47 & 201.4 & 0.0007823 & 0.04478 & 2166 & 117.3 & 1.3 & 975.6 & 0.2105371 \\
\hline 86.47 & 201.4 & 0.0007823 & $\begin{array}{l}0.04478 \\
0.0478\end{array}$ & 2166 & 117.3 & 1.3 & 975.6 & 0.2105371 \\
\hline 86.47 & 201.4 & 0.0007823 & 0.04478 & 2166 & 117.3 & 1.3 & 975.6 & 0.2105371 \\
\hline 86.47 & 201.4 & 0.0007823 & 0.04478 & 2166 & 117.3 & 1.3 & 975.6 & 0.2105371 \\
\hline 86.47 & 199.5 & 0.0007872 & 0.04166 & 2118 & 118.4 & 3.7 & 974.2 & 0.1881997 \\
\hline $\begin{array}{l}8.47 \\
86.47\end{array}$ & 199.5 & 0.0007872 & $\begin{array}{l}0.041100 \\
0.04166\end{array}$ & 2118 & $\begin{array}{l}118.4 \\
118.4\end{array}$ & $\begin{array}{l}3.1 \\
3.7\end{array}$ & $\begin{array}{l}9 / 4.2 \\
974.2\end{array}$ & 0.1881997 \\
\hline 86.47 & 200 & 0.000786 & 0.0424 & 2130 & 118.1 & 3.1 & 974.5 & 0.1935387 \\
\hline 86.47 & 199.5 & 0.0007872 & 0.04166 & 2118 & 118.4 & 3.7 & 974.2 & 0.1881997 \\
\hline 86.47 & 200 & 0.000786 & 0.0424 & 2130 & 118.1 & 3.1 & 974.5 & 0.1935387 \\
\hline $\begin{array}{l}80.41 \\
86.47\end{array}$ & 201.4 & 0.0007823 & $\begin{array}{l}0.0424 \\
0.04478\end{array}$ & 2166 & $\begin{array}{l}111.1 \\
117.3\end{array}$ & $\begin{array}{l}.1 .1 \\
1.3\end{array}$ & $\begin{array}{l}974.5 \\
975.6\end{array}$ & $\begin{array}{l}.1103538 \\
0.2105371\end{array}$ \\
\hline 86.47 & 201.4 & 0.0007823 & 0.04478 & 2166 & 117.3 & 1.3 & 975.6 & 0.2105371 \\
\hline 86.47 & 200.4 & 0.0007848 & 0.04316 & 2141 & 117.8 & 2.5 & 974.9 & 0.19902 \\
\hline 86.47 & 201.4 & 0.0007823 & 0.04478 & 2166 & 117.3 & 1.3 & 975.6 & 0.2105371 \\
\hline $\begin{array}{l}8.41 \\
86.47\end{array}$ & 200.9 & 0.0007836 & $\begin{array}{l}0.044396 \\
0.0436\end{array}$ & $\begin{array}{l}2100 \\
2153\end{array}$ & 117.5 & $\begin{array}{l}1.3 \\
1.9\end{array}$ & 975.3 & 0.2047004 \\
\hline 86.47 & 200 & 0.000786 & 0.0424 & 2130 & 118.1 & 3.1 & 974.5 & 0.1935387 \\
\hline 86.47 & 198.8 & 0.0007896 & 0.04025 & 2095 & 118.9 & 4.8 & 973.5 & 0.1787366 \\
\hline 86.47 & 200 & 0.000786 & 0.0424 & 2130 & 118.1 & $\begin{array}{l}\text { l.o } \\
3.1\end{array}$ & 974.5 & 0.1935387 \\
\hline
\end{tabular}

\section{R22 with SUS300 mineral oil}

$\begin{array}{lllllll}3.6 & 133 & 0.6 & 800 & 0.15 & 0.84 & 450 \\ 8.7 & 197 & 0.6 & 800 & 0.15 & 0.8 & 500 \\ 8.4 & 199 & 0.6 & 800 & 0.14 & 0.74 & 480 \\ 9.9 & 201 & 0.6 & 800 & 0.15 & 0.82 & 480 \\ 19.2 & 289 & 0.6 & 800 & 0.16 & 0.82 & 490 \\ 20.4 & 301 & 0.6 & 800 & 0.15 & 0.8 & 490 \\ 21.7 & 305 & 0.6 & 800 & 0.15 & 0.81 & 480 \\ 38 & 387 & 0.6 & 800 & 0.17 & 0.81 & 500 \\ 37.1 & 390 & 0.6 & 800 & 0.18 & 0.81 & 510 \\ 36.9 & 397 & 0.6 & 800 & 0.2 & 0.84 & 550 \\ 36.6 & 401 & 0.6 & 800 & 0.17 & 0.75 & 530 \\ 3.8 & 126 & 1.3 & 800 & 0.17 & 0.85 & 500 \\ 4.2 & 127 & 1.3 & 800 & 0.17 & 0.84 & 500 \\ 4.2 & 130 & 1.3 & 800 & 0.17 & 0.8 & 490 \\ 8.7 & 195 & 1.3 & 800 & 0.14 & 0.83 & 500 \\ 9.1 & 196 & 1.3 & 800 & 0.13 & 0.77 & 490 \\ 10.4 & 201 & 1.3 & 800 & 0.14 & 0.79 & 500 \\ 18 & 268 & 1.3 & 800 & 0.19 & 0.8 & 550 \\ 18.7 & 276 & 1.3 & 800 & 0.18 & 0.78 & 550 \\ 18.7 & 279 & 1.3 & 800 & 0.18 & 0.74 & 540 \\ 31.3 & 386 & 1.3 & 800 & 0.18 & 0.78 & 580 \\ 30.7 & 388 & 1.3 & 800 & 0.18 & 0.76 & 580 \\ 33 & 388 & 1.3 & 800 & 0.18 & 0.79 & 560\end{array}$

\begin{tabular}{ll}
86.47 & 204.8 \\
86.47 & 202.3 \\
86.47 & 203.3 \\
86.47 & 203.3 \\
86.47 & 202.8 \\
86.47 & 202.8 \\
86.47 & 203.3 \\
86.47 & 202.3 \\
86.47 & 201.9 \\
86.47 & 200 \\
86.47 & 200.9 \\
86.47 & 202.3 \\
86.47 & 202.3 \\
86.47 & 202.8 \\
86.47 & 202. \\
86.47 & 202.8 \\
86.47 & 202.3 \\
86.47 & 200 \\
86.47 & 200 \\
86.47 & 200.4 \\
86.47 & 198.8 \\
86.47 & 198.8 \\
86.47 & 199 \\
\hline
\end{tabular}

$\begin{array}{lll}0.0007734 & 0.05154 & 2260\end{array}$

$\begin{array}{llll}115.2 & -3.1 & 979.9 & 0.3980046 \\ 116.7 & 0.1 & 977.8 & 0.3453407 \\ 116.1 & -1.1 & 978.6 & 0.3650592 \\ 116.1 & -1.1 & 978.6 & 0.3650592 \\ 116.4 & -0.5 & 978.2 & 0.3551921 \\ 116.4 & -0.5 & 978.2 & 0.3551921 \\ 116.1 & -1.1 & 978.6 & 0.3650592 \\ 116.7 & 0.1 & 977.8 & 0.3553407 \\ 117 & 0.7 & 977.5 & 0.3358605 \\ 118.1 & 3.1 & 976 & 0.300252 \\ 117.5 & 1.9 & 976.8 & 0.3175372 \\ 116.7 & 0.1 & 977.8 & 0.3453407 \\ 116.7 & 0.1 & 977.8 & 0.3453407 \\ 116.4 & -0.5 & 978.2 & 0.3551921 \\ 116.7 & 0.1 & 977.8 & 0.3453407 \\ 116.4 & -0.5 & 978.2 & 0.3551921 \\ 116.7 & 0.1 & 977.8 & 0.3453407 \\ 118.1 & 3.1 & 976 & 0.300252 \\ 118.1 & 3.1 & 976 & 0.300252 \\ 117.8 & 2.5 & 976.4 & 0.3087923 \\ 118.9 & 4.8 & 975 & 0.2772973 \\ 118.9 & 4.8 & 975 & 0.2772973 \\ 118.4 & 3.7 & 975.7 & 0.2919755 \\ & & & \end{array}$




\begin{tabular}{|c|c|c|c|c|c|c|c|c|c|c|c|c|c|c|c|}
\hline 5.1 & 122 & 2.6 & 800 & 0.15 & 0.78 & 470 & 86.47 & 203.8 & 0.000776 & 0.04941 & 2231 & 115.8 & -1.7 & 979 & 0.3749421 \\
\hline 4 & 124 & 2.6 & 800 & 0.15 & 0.78 & 490 & 86.47 & 202.8 & 0.0007786 & 0.04745 & 2204 & 116.4 & -0.5 & 978.2 & 0.3551921 \\
\hline 4.4 & 137 & 2.6 & 800 & 0.14 & 0.71 & 490 & 86.47 & 202.8 & 0.0007786 & 0.04745 & 2204 & 116.4 & -0.5 & 978.2 & 0.3551921 \\
\hline 10.2 & 194 & 2.6 & 800 & 0.16 & 0.77 & 540 & 86.47 & 200.4 & 0.0007848 & 0.04316 & 2141 & 117.8 & 2.5 & 976.4 & 0.3087923 \\
\hline 8.9 & 197 & 2.6 & 800 & 0.15 & 0.75 & 530 & 86.47 & 200.9 & 0.0007836 & 0.04396 & 2153 & 117.5 & 1.9 & 976.8 & 0.3175372 \\
\hline 10.9 & 198 & 2.6 & 800 & 0.17 & 0.76 & 500 & 86.47 & 202.3 & 0.0007798 & 0.04653 & 2191 & 116.7 & 0.1 & 977.8 & 0.3453407 \\
\hline 10.9 & 203 & 2.6 & 800 & 0.16 & 0.82 & 510 & 86.47 & 201.9 & 0.0007811 & 0.04564 & 2178 & 117 & 0.7 & 977.5 & 0.3358605 \\
\hline 21.1 & 296 & 2.6 & 800 & 0.18 & 0.81 & 550 & 86.47 & 200 & 0.000786 & $\begin{array}{l}0.0424 \\
\end{array}$ & 2130 & 118.1 & 3.1 & 976 & 0.300252 \\
\hline 21.7 & 297 & 2.6 & 800 & 0.18 & 0.81 & 560 & 86.47 & 199.5 & 0.0007872 & 0.04166 & 2118 & 118.4 & 3.7 & 975.7 & 0.2919755 \\
\hline 36.9 & 389 & 2.6 & 800 & 0.17 & 0.81 & 530 & 86.47 & 200.9 & 0.0007836 & 0.04396 & 2153 & 117.5 & 1.9 & 976.8 & 0.3175372 \\
\hline 38.1 & 390 & 2.6 & 800 & 0.22 & 0.84 & 560 & 86.47 & 199.5 & 0.0007872 & 0.04166 & 2118 & 118.4 & 3.7 & 975.7 & 0.2919755 \\
\hline $\begin{array}{l}30.1 \\
37.4\end{array}$ & 396 & 2.6 & 800 & 0.19 & $\begin{array}{l}0.04 \\
0.8\end{array}$ & 540 & $\begin{array}{l}80.47 \\
86.47\end{array}$ & 200.4 & 0.0007848 & 0.04316 & 2141 & $\begin{array}{l}117.4 \\
117.8\end{array}$ & 2.5 & 976.4 & 0.3087923 \\
\hline 35.8 & 398 & 2.6 & 800 & 0.16 & 0.78 & 520 & 86.47 & 201.4 & 0.0007823 & 0.04478 & 2166 & 117.3 & 1.3 & 977.1 & 0.3265157 \\
\hline 5.3 & 126 & 5 & 800 & 0.16 & 0.76 & 530 & $\begin{array}{l}80.47 \\
86.47\end{array}$ & 200.9 & 0.0007836 & 0.04396 & 2153 & 117.5 & 1.9 & 976.8 & 0.3175372 \\
\hline 4.7 & 130 & 5 & 800 & 0.15 & 0.71 & 520 & 86.47 & 201.4 & 0.0007823 & 0.04478 & 2166 & 117.3 & 1.3 & 977.1 & 0.3265157 \\
\hline $\begin{array}{l}4.1 \\
6.2\end{array}$ & 136 & 5 & 800 & 0.15 & 0.84 & 510 & $\begin{array}{l}80.47 \\
86.47\end{array}$ & $\begin{array}{l}201.9 \\
\end{array}$ & 0.0007811 & 0.04564 & 2178 & 117 & 0.7 & 977.5 & 0.3358605 \\
\hline 6.3 & 144 & 5 & 800 & 0.11 & 0.84 & 470 & 86.47 & 203.8 & 0.000776 & 0.04941 & 2231 & 115.8 & -1.7 & 979 & 0.3749421 \\
\hline $\begin{array}{l}14.1 \\
14.1\end{array}$ & 211 & 5 & 800 & 0.1 & $\begin{array}{l}0.04 \\
0.8\end{array}$ & 530 & $\begin{array}{l}80.47 \\
86.47\end{array}$ & 200.9 & 0.0007836 & 0.04396 & 2153 & 117.5 & 1.9 & 976.8 & 0.3175372 \\
\hline 16.2 & 217 & 5 & 800 & 0.14 & 0.84 & 530 & 86.47 & 200.9 & 0.0007836 & 0.04396 & 2153 & 117.5 & 1.9 & 976.8 & 0.3175372 \\
\hline $\begin{array}{l}10.2 \\
16.4\end{array}$ & 218 & 5 & 800 & 0.13 & $\begin{array}{l}0.04 \\
0.82\end{array}$ & 520 & $\begin{array}{l}80.47 \\
86.47\end{array}$ & 201.4 & 0.0007823 & 0.04478 & 2166 & 117.3 & 1.3 & 977.1 & 0.3265157 \\
\hline 23.1 & 278 & 5 & 800 & 0.2 & 0.83 & 560 & 86.47 & 199.5 & 0.0007872 & 0.04166 & 2118 & 118.4 & 3.7 & 975.7 & 0.2919755 \\
\hline 23.7 & 278 & 5 & 800 & 0.19 & 0.81 & 530 & $\begin{array}{l}80.47 \\
86.47\end{array}$ & 200.9 & 0.0007836 & 0.04396 & 2153 & $\begin{array}{l}117.4 \\
117.5\end{array}$ & 1.9 & 976.8 & 0.3175372 \\
\hline 22.3 & 287 & 5 & 800 & 0.19 & 0.77 & 550 & 86.47 & 200 & 0.000786 & 0.0424 & 2130 & 118.1 & 3.1 & 976 & 0.300252 \\
\hline 25.2 & 302 & 5 & 800 & $\begin{array}{l}0.19 \\
0.17\end{array}$ & 0.76 & 520 & $\begin{array}{l}80.47 \\
86.47\end{array}$ & 201.4 & 0.0007823 & $\begin{array}{l}0.0424 \\
0.04478\end{array}$ & 2166 & $\begin{array}{l}118.1 \\
117.3\end{array}$ & $\begin{array}{l}3.1 \\
1.3\end{array}$ & $\begin{array}{l}9710 \\
977.1\end{array}$ & $\begin{array}{l}0.3265157 \\
0.32\end{array}$ \\
\hline 33.9 & 360 & 5 & 800 & 0.21 & 0.81 & 570 & 86.47 & 199 & 0.0007884 & 0.04094 & 2107 & 118.6 & 4.2 & 975.4 & 0.2852313 \\
\hline 32.8 & 372 & 5 & 800 & 0.19 & 0.75 & 560 & 86.47 & 199.5 & 0.0007872 & 0.04166 & 2118 & 118.4 & 3.7 & 975.7 & 0.2919755 \\
\hline 34.8 & 378 & 5 & 800 & 0.18 & 0.71 & 540 & 86.47 & 200.4 & 0.0007848 & 0.04316 & 2141 & 117.8 & 2.5 & 976.4 & 0.3087923 \\
\hline
\end{tabular}

Micro-fin 3/8 inch, condensation

R12 with SUS150 naphthenic oil

$\begin{array}{lllllll}0.76 & 122 & 1.3 & 800 & 0.81 & 0.11 & 949 \\ 0.65 & 123 & 1.3 & 800 & 0.81 & 0.12 & 953 \\ 1.26 & 129 & 1.3 & 800 & 0.79 & 0.11 & 944 \\ 1.14 & 120 & 1.3 & 800 & 0.87 & 0.1 & 959 \\ 2.97 & 207 & 1.3 & 800 & 0.8 & 0.06 & 952 \\ 3.03 & 205 & 1.3 & 800 & 0.85 & 0.08 & 958 \\ 6.38 & 302 & 1.3 & 800 & 0.81 & 0.08 & 943 \\ 6.08 & 295 & 1.3 & 800 & 0.83 & 0.07 & 949 \\ 8.54 & 366 & 1.3 & 800 & 0.81 & 0.08 & 938 \\ 8.7 & 365 & 1.3 & 800 & 0.82 & 0.08 & 940 \\ 0.74 & 130 & 2.4 & 800 & 0.8 & 0.06 & 939 \\ 0.91 & 131 & 2.4 & 800 & 0.79 & 0.07 & 943 \\ 2.76 & 203 & 2.4 & 800 & 0.82 & 0.07 & 944 \\ 2.77 & 205 & 2.4 & 800 & 0.81 & 0.07 & 946 \\ 6.08 & 299 & 2.4 & 800 & 0.83 & 0.07 & 947 \\ 5.99 & 299 & 2.4 & 800 & 0.84 & 0.07 & 946 \\ 10.08 & 382 & 2.4 & 800 & 0.82 & 0.08 & 928 \\ 10.57 & 381 & 2.4 & 800 & 0.83 & 0.09 & 926 \\ 1.12 & 134 & 4.9 & 800 & 0.81 & 0.08 & 930 \\ 0.93 & 134 & 4.9 & 800 & 0.82 & 0.08 & 932 \\ 2.78 & 204 & 4.9 & 800 & 0.81 & 0.07 & 948 \\ 2.74 & 199 & 4.9 & 800 & 0.83 & 0.07 & 949 \\ 2.72 & 199 & 4.9 & 800 & 0.83 & 0.07 & 949 \\ 6.04 & 296 & 4.9 & 800 & 0.81 & 0.07 & 938 \\ 6.13 & 297 & 4.9 & 800 & 0.81 & 0.07 & 938 \\ 10.93 & 368 & 4.9 & 800 & 0.81 & 0.11 & 889 \\ 11.44 & 368 & 4.9 & 800 & 0.81 & 0.11 & 890\end{array}$

$\begin{array}{lllllllll}120.91 & 125.9 & 0.0007955 & 0.01798 & 1654 & 132.9 & 39.6 & 952.6 & 0.0352089 \\ 120.91 & 125.7 & 0.0007959 & 0.0179 & 1651 & 133 & 39.7 & 952.6 & 0.0350274 \\ 120.91 & 126 & 0.0007949 & 0.01808 & 1657 & 132.8 & 39.4 & 952.8 & 0.0355869 \\ 120.91 & 125.6 & 0.0007966 & 0.01779 & 1647 & 133.2 & 39.6 & 952.6 & 0.0352089 \\ 120.91 & 125.8 & 0.0007958 & 0.01792 & 1652 & 133 & 39.7 & 952.6 & 0.0350274 \\ 120.91 & 125.6 & 0.0007965 & 0.01781 & 1648 & 133.1 & 39.2 & 952.9 & 0.0358667 \\ 120.91 & 126.1 & 0.0007948 & 0.0181 & 1658 & 132.8 & 39.3 & 952.8 & 0.0357684 \\ 120.91 & 125.9 & 0.0007955 & 0.01798 & 1654 & 132.9 & 39.6 & 952.6 & 0.0352089 \\ 120.91 & 126.2 & 0.0007942 & 0.0182 & 1661 & 132.7 & 39.1 & 952.9 & 0.0360483 \\ 120.91 & 126.1 & 0.0007945 & 0.01816 & 1660 & 132.7 & 39.2 & 952.9 & 0.0358667 \\ 120.91 & 126.1 & 0.0007944 & 0.01818 & 1660 & 132.7 & 39.2 & 952.9 & 0.0358667 \\ 120.91 & 126.1 & 0.0007948 & 0.0181 & 1658 & 132.8 & 39.3 & 952.8 & 0.0357684 \\ 120.91 & 126 & 0.0007949 & 0.01808 & 1657 & 132.8 & 39.4 & 952.8 & 0.0355869 \\ 120.91 & 125.9 & 0.0007951 & 0.01804 & 1656 & 132.9 & 39.4 & 952.8 & 0.0355869 \\ 120.91 & 125.9 & 0.0007952 & 0.01802 & 1655 & 132.9 & 39.5 & 952.7 & 0.0353979 \\ 120.91 & 125.9 & 0.0007951 & 0.01804 & 1656 & 132.9 & 39.4 & 952.8 & 0.0355869 \\ 120.91 & 126.5 & 0.0007931 & 0.01841 & 1668 & 132.4 & 38.7 & 953.2 & 0.036707 \\ 120.91 & 126.6 & 0.0007929 & 0.01845 & 1669 & 132.4 & 38.6 & 953.2 & 0.0368888 \\ 120.91 & 126.4 & 0.0007934 & 0.01837 & 1666 & 132.5 & 38.8 & 953.1 & 0.0366085 \\ 120.91 & 126.3 & 0.0007936 & 0.01833 & 1665 & 132.5 & 38.9 & 953.1 & 0.0364268 \\ 120.91 & 125.9 & 0.0007954 & 0.018 & 1654 & 132.9 & 39.5 & 952.7 & 0.0353979 \\ 120.91 & 125.9 & 0.0007955 & 0.01798 & 1654 & 132.9 & 39.5 & 952.7 & 0.0353979 \\ 120.91 & 125.9 & 0.0007955 & 0.01798 & 1654 & 132.9 & 39.6 & 952.6 & 0.0352089 \\ 120.91 & 126.2 & 0.0007942 & 0.0182 & 1661 & 132.7 & 39 & 953 & 0.0362375 \\ 120.91 & 126.2 & 0.0007942 & 0.0182 & 1661 & 132.7 & 39.1 & 952.9 & 0.0360483 \\ 120.91 & 127.6 & 0.0007888 & 0.01925 & 1695 & 131.5 & 37 & 954.2 & 0.0397887 \\ 120.91 & 127.7 & 0.0007889 & 0.01923 & 1694 & 131.5 & 37.1 & 954.1 & 0.0395983\end{array}$

\section{R12 with SUS300 naphthenic oil}

\begin{tabular}{|c|c|c|c|c|c|}
\hline 0.87 & 131 & 1.2 & 800 & 0.8 & 0.07 \\
\hline 0.89 & 130 & 1.2 & 800 & 0.8 & 0.08 \\
\hline 2.81 & 203 & 1.2 & 800 & 0.82 & 0.1 \\
\hline 2.93 & 203 & 1.2 & 800 & 0.82 & 0.1 \\
\hline 2.67 & 201 & 1.2 & 800 & 0.79 & 0.1 \\
\hline 2.85 & 201 & 1.2 & 800 & 0.8 & 0.11 \\
\hline 6.53 & 303 & 1.2 & 800 & 0.83 & 0.11 \\
\hline 6.72 & 303 & 1.2 & 800 & 0.83 & 0.11 \\
\hline 10.26 & 375 & 1.2 & 800 & 0.81 & 0.09 \\
\hline 11.15 & 381 & 1.2 & 800 & 0.81 & 0.1 \\
\hline 10.41 & 373 & 1.2 & 800 & 0.81 & 0.12 \\
\hline 10.99 & 375 & 1.2 & 800 & 0.8 & 0.12 \\
\hline 0.9 & 125 & 2.4 & 800 & 0.84 & 0.07 \\
\hline 0.91 & 127 & 2.4 & 800 & 0.83 & 0.07 \\
\hline 2.81 & 197 & 2.4 & 800 & 0.83 & 0.08 \\
\hline 2.88 & 198 & 2.4 & 800 & 0.83 & 0.08 \\
\hline 6.19 & 297 & 2.4 & 800 & 0.82 & 0.07 \\
\hline 6.26 & 297 & 2.4 & 800 & 0.81 & 0.08 \\
\hline 6.45 & 296 & 2.4 & 800 & 0.81 & 0.09 \\
\hline 10.76 & 367 & 2.4 & 800 & 0.83 & 0.07 \\
\hline 9.79 & 382 & 2.4 & 800 & 0.81 & 0.07 \\
\hline 0.94 & 126 & 4.8 & 800 & 0.82 & 0.08 \\
\hline 0.97 & 126 & 4.8 & 800 & 0.82 & 0.08 \\
\hline 3.04 & 199 & 4.8 & 800 & 0.84 & 0.09 \\
\hline 3.22 & 200 & 4.8 & 800 & 0.83 & 0.08 \\
\hline 3.03 & 200 & 4.8 & 800 & 0.83 & 0.08 \\
\hline 2.86 & 206 & 4.8 & 800 & 0.8 & 0.07 \\
\hline 6.44 & 301 & 4.8 & 800 & 0.82 & 0.08 \\
\hline 6.5 & 302 & 4.8 & 800 & 0.82 & 0.09 \\
\hline 10.66 & 370 & 4.8 & 800 & 0.8 & 0.09 \\
\hline 10.3 & 369 & 4.8 & 800 & 0.8 & 0.09 \\
\hline
\end{tabular}

$\begin{array}{lllllllll}120.91 & 125.9 & 0.0007956 & 0.01796 & 1653 & 132.9 & 39.6 & 954.1 & 0.0546184 \\ 120.91 & 125.8 & 0.0007957 & 0.01794 & 1652 & 133 & 39.6 & 954.1 & 0.0546184 \\ 120.91 & 125.6 & 0.0007962 & 0.01785 & 1649 & 133.1 & 39.8 & 954 & 0.0541519 \\ 120.91 & 125.7 & 0.0007964 & 0.01783 & 1649 & 133.1 & 39.9 & 954 & 0.0538789 \\ 120.91 & 125.6 & 0.0007965 & 0.01781 & 1648 & 133.1 & 39.9 & 954 & 0.0538789 \\ 120.91 & 125.6 & 0.0007966 & 0.01779 & 1647 & 133.2 & 40 & 953.9 & 0.0536856 \\ 120.91 & 126.3 & 0.000794 & 0.01824 & 1662 & 132.6 & 39 & 954.5 & 0.056213 \\ 120.91 & 126.3 & 0.000794 & 0.01824 & 1662 & 132.6 & 39 & 954.5 & 0.056213 \\ 120.91 & 126.4 & 0.0009934 & 0.01837 & 1666 & 132.5 & 38.8 & 954.6 & 0.0567716 \\ 120.91 & 126.6 & 0.000793 & 0.01843 & 1668 & 132.4 & 38.7 & 954.7 & 0.0569658 \\ 120.91 & 126.1 & 0.0007944 & 0.01818 & 1660 & 132.7 & 39.1 & 954.4 & 0.055928 \\ 120.91 & 126.2 & 0.0007941 & 0.01822 & 1662 & 132.6 & 39.1 & 954.4 & 0.055928 \\ 120.91 & 126.2 & 0.0007941 & 0.01822 & 1662 & 132.6 & 39.1 & 954.4 & 0.055928 \\ 120.91 & 126.1 & 0.0007944 & 0.01818 & 1660 & 132.7 & 39.1 & 954.4 & 0.055928 \\ 120.91 & 125.8 & 0.0007957 & 0.01794 & 1652 & 133 & 39.6 & 954.1 & 0.0546184 \\ 120.91 & 125.9 & 0.0007956 & 0.01796 & 1653 & 132.9 & 39.6 & 954.1 & 0.0546184 \\ 120.91 & 126.2 & 0.0007941 & 0.01822 & 1662 & 132.6 & 39.1 & 954.4 & 0.055928 \\ 120.91 & 126 & 0.0007949 & 0.01808 & 1657 & 132.8 & 39.3 & 954.3 & 0.0554609 \\ 120.91 & 125.8 & 0.0007957 & 0.01794 & 1652 & 133 & 39.7 & 954.1 & 0.0544363 \\ 120.91 & 125.9 & 0.0007951 & 0.01804 & 1656 & 132.9 & 39.4 & 954.3 & 0.0551877 \\ 120.91 & 126.1 & 0.0007948 & 0.0181 & 1658 & 132.8 & 39.3 & 954.3 & 0.0554609 \\ 120.91 & 126.2 & 0.0007942 & 0.0182 & 1661 & 132.7 & 39.1 & 954.4 & 0.055928 \\ 120.91 & 126.3 & 0.000794 & 0.01824 & 1662 & 132.6 & 39.1 & 954.4 & 0.055928 \\ 120.91 & 126.7 & 0.0007924 & 0.01855 & 1673 & 132.3 & 38.4 & 954.9 & 0.0578103 \\ 120.91 & 126.7 & 0.0007925 & 0.01853 & 1672 & 132.3 & 38.4 & 954.9 & 0.0578103 \\ 120.91 & 126.1 & 0.0007947 & 0.01812 & 1658 & 132.8 & 39.3 & 954.3 & 0.0554609 \\ 120.91 & 125.9 & 0.0007952 & 0.01802 & 1655 & 132.9 & 39.4 & 954.3 & 0.0551877 \\ 120.91 & 126.2 & 0.0009941 & 0.01822 & 1662 & 132.6 & 39 & 954.5 & 0.056213 \\ 120.91 & 126.3 & 0.0007939 & 0.01827 & 1663 & 132.6 & 39 & 954.5 & 0.056213 \\ 120.91 & 126.8 & 0.0007919 & 0.01864 & 1675 & 132.2 & 38.2 & 955 & 0.0583696 \\ 120.91 & 126.9 & 0.0007917 & 0.01868 & 1677 & 132.1 & 38.1 & 955 & 0.0586432\end{array}$

\section{R134a with SUS169 ester-m oil}

\begin{tabular}{|c|c|c|c|c|c|}
\hline 1.49 & 127 & 0.5 & 500 & 0.86 & 0.13 \\
\hline 1.44 & 129 & 0.5 & 500 & 0.85 & 0.11 \\
\hline 1.41 & 127 & 0.5 & 500 & 0.86 & 0.12 \\
\hline 2.98 & 196 & 0.5 & 500 & 0.84 & 0.13 \\
\hline 2.87 & 197 & 0.5 & 500 & 0.83 & 0.12 \\
\hline 8.35 & 300 & 0.5 & 500 & 0.85 & 0.15 \\
\hline 7.58 & 298 & 0.5 & 500 & 0.86 & 0.14 \\
\hline 7.94 & 300 & 0.5 & 500 & 0.86 & 0.14 \\
\hline 12.72 & 378 & 0.5 & 500 & 0.85 & 0.14 \\
\hline 12.79 & 378 & 0.5 & 500 & 0.84 & 0.13 \\
\hline 1.5 & 129 & 1 & 500 & 0.83 & 0.12 \\
\hline 1.76 & 129 & 1 & 500 & 0.85 & 0.13 \\
\hline 3.23 & 197 & 1 & 500 & 0.87 & 0.13 \\
\hline 3.96 & 195 & 1 & 500 & 0.88 & 0.14 \\
\hline
\end{tabular}

$\begin{array}{llllllllll}1041 & 102.03 & 162.3 & 0.0008751 & 0.0195 & 1684 & 129 & 40.8 & 952.1 & 0.0357159 \\ 1040 & 102.03 & 162.3 & 0.000875 & 0.01952 & 1685 & 129 & 40.8 & 952.1 & 0.0357159 \\ 1039 & 102.03 & 162.4 & 0.0008749 & 0.01953 & 1686 & 128.9 & 40.8 & 952.1 & 0.0357159 \\ 992 & 102.03 & 164.2 & 0.0008693 & 0.02051 & 1726 & 128 & 39.1 & 953.1 & 0.038607 \\ 991 & 102.03 & 164.2 & 0.0008692 & 0.02053 & 1727 & 128 & 39 & 953.2 & 0.0387968 \\ 1008 & 102.03 & 163.6 & 0.0008712 & 0.02017 & 1712 & 128.3 & 39.7 & 952.8 & 0.0375481 \\ 1010 & 102.03 & 163.5 & 0.0008715 & 0.02013 & 1711 & 128.4 & 39.7 & 952.8 & 0.0375841 \\ 1008 & 102.03 & 163.6 & 0.0008712 & 0.02017 & 1712 & 128.3 & 39.7 & 952.8 & 0.0375841 \\ 1004 & 102.03 & 163.7 & 0.0008708 & 0.02026 & 1716 & 128.2 & 39.6 & 952.8 & 0.0377656 \\ 1000 & 102.03 & 163.8 & 0.0008703 & 0.02034 & 1719 & 128.2 & 39.4 & 953 & 0.0381448 \\ 1000 & 102.03 & 163.8 & 0.00087703 & 0.02034 & 1719 & 128.2 & 39.4 & 953 & 0.0381448 \\ 1000 & 102.03 & 163.8 & 0.0008703 & 0.02034 & 1719 & 128.2 & 39.4 & 953 & 0.0381448 \\ 1017 & 102.03 & 163.2 & 0.0008723 & 0.01998 & 1705 & 128.5 & 40.1 & 952.5 & 0.0369253 \\ 1019 & 102.03 & 163.1 & 0.0008725 & 0.01994 & 1703 & 128.5 & 40.1 & 952.5 & 0.0369253\end{array}$



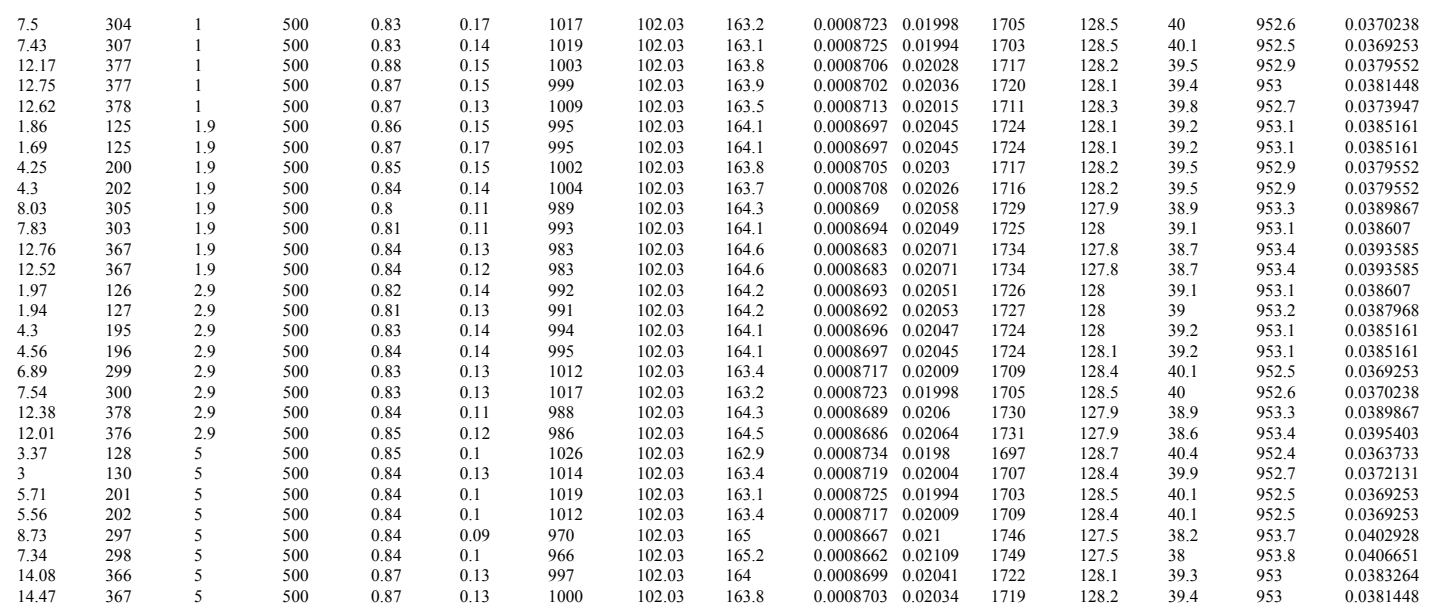

\section{R134a with SUS369 ester-m oil}

\begin{tabular}{|c|c|c|c|c|c|}
\hline & 123 & 0.6 & 500 & & \\
\hline 0.69 & 124 & 0.6 & 500 & 0.88 & 0.12 \\
\hline 3.23 & 193 & 0.6 & 500 & 0.87 & 0.11 \\
\hline 2.96 & 193 & 0.6 & 500 & 0.86 & 0.1 \\
\hline 6.5 & 294 & 0.6 & 500 & 0.84 & 0.1 \\
\hline 6.95 & 296 & 0.6 & 500 & 0.84 & 0.1 \\
\hline 12.81 & 370 & 0.6 & 500 & 0.81 & 0.15 \\
\hline 12.8 & 371 & 0.6 & 500 & 0.81 & 0.15 \\
\hline 0.69 & 122 & 1.1 & 500 & 0.87 & 0.11 \\
\hline 0.67 & 122 & 1.1 & 500 & 0.85 & 0.1 \\
\hline 2.78 & 196 & 1.1 & 500 & 0.85 & 0.12 \\
\hline 3.06 & 195 & 1.1 & 500 & 0.85 & 0.12 \\
\hline 7.42 & 300 & 1.1 & 500 & 0.84 & 0.14 \\
\hline 6.5 & 299 & 1.1 & 500 & 0.84 & 0.14 \\
\hline 11.82 & 371 & 1.1 & 500 & 0.82 & 0.15 \\
\hline 11.98 & 367 & 1.1 & 500 & 0.83 & 0.15 \\
\hline 11.43 & 364 & 1.1 & 500 & 0.84 & 0.15 \\
\hline 1 & 128 & 2.4 & 500 & 0.84 & 0.09 \\
\hline 0.99 & 129 & 2.4 & 500 & 0.84 & 0.09 \\
\hline 3.08 & 203 & 2.4 & 500 & 0.83 & 0.1 \\
\hline 2.95 & 198 & 2.4 & 500 & 0.84 & 0.11 \\
\hline 2.88 & 198 & 2.4 & 500 & 0.84 & 0.11 \\
\hline 2.75 & 198 & 2.4 & 500 & 0.84 & 0.11 \\
\hline 6.68 & 299 & 2.4 & 500 & 0.82 & 0.12 \\
\hline 6.91 & 300 & 2.4 & 500 & 0.82 & 0.13 \\
\hline 10.83 & 374 & 2.4 & 500 & 0.84 & 0.12 \\
\hline 10.97 & 374 & 2.4 & 500 & 0.84 & 0.13 \\
\hline 0.31 & 118 & 5 & 500 & 0.83 & 0.12 \\
\hline 0.34 & 119 & 5 & 500 & 0.82 & 0.11 \\
\hline 3.32 & 203 & 5 & 500 & 0.83 & 0.1 \\
\hline 3.28 & 204 & 5 & 500 & 0.83 & 0.1 \\
\hline 7.88 & 303 & 5 & 500 & 0.83 & 0.13 \\
\hline 7.33 & 304 & 5 & 500 & 0.83 & 0.12 \\
\hline 12.05 & 368 & 5 & 500 & 0.83 & 0.13 \\
\hline 12.4 & 368 & 5 & 500 & 0.84 & 0.14 \\
\hline 12.55 & 366 & 5 & 500 & 0.84 & 0.14 \\
\hline
\end{tabular}

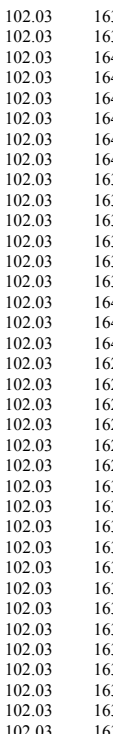

$\begin{array}{lll}0.0008713 & 0.02015 & 1711\end{array}$ $\begin{array}{lll}0.0008717 & 0.02009 & 1709\end{array}$ $\begin{array}{lll}0.0008692 & 0.02053 & 1727\end{array}$ $\begin{array}{lll}0.0008693 & 0.02051 & 1726\end{array}$ $\begin{array}{lll}0.0008687 & 0.02062 & 1731\end{array}$ $\begin{array}{lll}0.0008699 & 0.02041 & 1732 \\ 0.0008689 & 0.0206 & 1730\end{array}$ $0.0008689-0.0206$ $\begin{array}{lll}0.0008689 & 0.0206 & 1730 \\ 0.0008704 & 0.02032 & 1718\end{array}$ $\begin{array}{lll}0.0008704 & 0.02032 & 1718 \\ 0.0008706 & 0.02028 & 1717\end{array}$ $\begin{array}{lll}0.0008705 & 0.0203 & 1717\end{array}$ $\begin{array}{lll}0.0008708 & 0.02026 & 1716\end{array}$ $\begin{array}{lll}0.0008704 & 0.02032 & 1718 \\ 0.0008708 & 0.02026 & 1716\end{array}$ $0.0008677 \quad 0.02082$ 0.00086860 .02064 $\begin{array}{lll}0.0008677 & 0.02082 & 1739 \\ 0.0008687 & 0.02064-1731\end{array}$ $\begin{array}{llll}0.0008744 & 0.01961 & 1698\end{array}$ $\begin{array}{lll}0.0008742 & 0.01966 & 1691\end{array}$ $\begin{array}{lll}0.0008735 & 0.01978 & 1696 \\ 0.000836 & 0.01976 & 169\end{array}$ $\begin{array}{lll}0.0008736 & 0.01976 & 1695\end{array}$ $\begin{array}{lll}0.0008735 & 0.01978 & 1696\end{array}$ $\begin{array}{lll}0.0008732 & 0.01982\end{array}$ $\begin{array}{lll}0.0008711 & 0.02019\end{array}$ $\begin{array}{lll}0.0008711 & 0.02019 & 1713\end{array}$ $\begin{array}{lll}0.000873 & 0.01986 & 1699 \\ 0.0008729 & 0.01988 & 1700\end{array}$ $\begin{array}{lll}0.0008729 & 0.01988 & 1700 \\ 0.0008713 & 0.02015 & 1711\end{array}$ $\begin{array}{ll}0.0008713 & 0.02015 \\ 0.0008712 & 0.02017\end{array}$ $\begin{array}{ll}0.0008712 & 0.02017 \\ 0.0008719 & 0.02004\end{array}$ $\begin{array}{ll}0.0008719 & 0.02004\end{array}$ $\begin{array}{ll}0.0008716 & 0.02011\end{array}$ $\begin{array}{ll}0.0008721 & 0.02002 \\ 0.0008705 & 0.0203\end{array}$ $0.0008709 \quad 0.0202$ $\begin{array}{lll}0.0008709 & 0.02023 & 1715 \\ 0.0008712 & 0.02017 & 1712\end{array}$

$\begin{array}{llll}128.3 & 39.7 & 954.8 & 0.0670058 \\ 128.4 & 39.9 & 954.7 & 0.0663537 \\ 128 & 39 & 955.2 & 0.0691605 \\ 128 & 39.1 & 955.1 & 0.0688723 \\ 127.9 & 38.9 & 955.3 & 0.06954 \\ 128.1 & 39.3 & 955 & 0.0682195 \\ 127.9 & 38.9 & 955.3 & 0.06954 \\ 127.9 & 38.9 & 955.3 & 0.06954 \\ 128.2 & 39.4 & 955 & 0.0679459 \\ 128.2 & 39.5 & 954.9 & 0.0675669 \\ 128.2 & 39.5 & 954.9 & 0.0675669 \\ 128.2 & 39.5 & 954.9 & 0.0675669 \\ 128.2 & 39.4 & 955 & 0.0679459 \\ 128.2 & 39.6 & 954.8 & 0.0672792 \\ 127.7 & 38.5 & 955.5 & 0.0708473 \\ 127.9 & 38.8 & 955.3 & 0.0698138 \\ 127.9 & 38.9 & 955.3 & 0.06954 \\ 128.9 & 40.2 & 954.5 & 0.0654148 \\ 128.8 & 40.6 & 954.2 & 0.0641901 \\ 128.7 & 40.4 & 954.4 & 0.0648546 \\ 128.7 & 40.4 & 954.4 & 0.0648546 \\ 128.7 & 40.4 & 954.4 & 0.0648546 \\ 128.7 & 40.3 & 954.4 & 0.0651279 \\ 128.3 & 39.7 & 954.8 & 0.0670058 \\ 128.3 & 39.7 & 954.8 & 0.0670058 \\ 128.6 & 40.2 & 954.5 & 0.0654148 \\ 128.6 & 40.2 & 954.5 & 0.0654148 \\ 128.3 & 39.7 & 954.8 & 0.0670058 \\ 128.3 & 39.7 & 954.8 & 0.0670058 \\ 128.4 & 39.9 & 954.7 & 0.0663537 \\ 128.4 & 40 & 954.6 & 0.0660664 \\ 128.4 & 39.8 & 954.7 & 0.0666271 \\ 128.5 & 40 & 954.6 & 0.0660664 \\ 128.2 & 39.5 & 954.9 & 0.0675669 \\ 128.3 & 39.6 & 954.8 & 0.0672792 \\ 128 . & 39.7 & 954.8 & 0.0670058\end{array}$

\section{R134a with SUS150 ester-b oil}
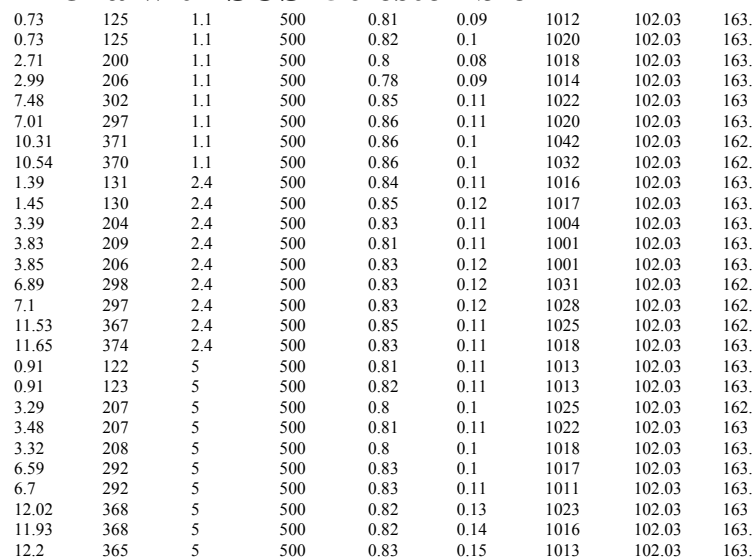

$\begin{array}{lll}0.0008717 & 0.02009 & 1709\end{array}$

$\begin{array}{llll}128.4 & 40.2 & 952.3 & 0.0342799 \\ 128.6 & 40.1 & 952.3 & 0.0343706 \\ 128.5 & 40.1 & 952.3 & 0.0343706 \\ 128.4 & 40 & 952.4 & 0.0345592 \\ 128.6 & 40.2 & 952.3 & 0.0342799 \\ 128.5 & 40.1 & 952.3 & 0.0343706 \\ 129 & 40.9 & 951.9 & 0.0331638 \\ 128.8 & 40.5 & 952.1 & 0.0338122 \\ 128.5 & 40 & 952.4 & 0.0345592 \\ 128.5 & 40.1 & 952.3 & 0.0343706 \\ 128.2 & 39.5 & 952.7 & 0.0353979 \\ 128.2 & 39.4 & 955.8 & 0.0355869 \\ 128.2 & 39.4 & 952.8 & 0.0355869 \\ 128.8 & 40.5 & 952.1 & 0.0338122 \\ 128.7 & 40.4 & 952.2 & 0.03391 \\ 128.7 & 40.3 & 952.2 & 0.0340913 \\ 128.5 & 40.1 & 952.3 & 0.0343706 \\ 128.4 & 39.9 & 952.5 & 0.0347479 \\ 128.4 & 39.9 & 955.5 & 0.0344779 \\ 128.7 & 40.3 & 952.2 & 0.0340913 \\ 128.6 & 40.2 & 952.3 & 0.0342799 \\ 128.5 & 40.1 & 952.3 & 0.0343706 \\ 128.5 & 40 & 952.4 & 0.0345592 \\ 128.4 & 39.8 & 952.5 & 0.0349294 \\ 128.6 & 40.2 & 955.3 & 0.0342799 \\ 128.5 & 40 & 952.4 & 0.0345592 \\ 128.4 & 39.9 & 952.5 & 0.0347479\end{array}$

\section{R22 with SUS150 mineral oil}

$\begin{array}{lllllll}8.4 & 296 & 1.3 & 800 & 0.89 & 0.16 & 1570 \\ 7.2 & 301 & 1.3 & 800 & 0.86 & 0.07 & 1590 \\ 7 & 306 & 1.3 & 800 & 0.83 & 0.08 & 1550 \\ 12 & 395 & 1.3 & 800 & 0.85 & 0.12 & 152 \\ 11.7 & 398 & 1.3 & 800 & 0.84 & 0.11 & 153 \\ 11.8 & 400 & 1.3 & 800 & 0.84 & 0.12 & 1520 \\ 1.5 & 122 & 2.4 & 800 & 0.83 & 0.1 & 1540 \\ 1.4 & 124 & 2.4 & 800 & 0.82 & 0.08 & 1530 \\ 1.3 & 127 & 2.4 & 800 & 0.81 & 0.09 & 1530 \\ 3.4 & 199 & 2.4 & 800 & 0.86 & 0.16 & 156 \\ 3.6 & 201 & 2.4 & 800 & 0.84 & 0.14 & 156\end{array}$




\begin{tabular}{|c|c|c|c|c|c|c|c|c|c|c|c|c|c|c|c|}
\hline 3.3 & 202 & 2.4 & 800 & 0.81 & 0.09 & 1570 & 86.47 & 162.9 & 0.0008898 & 0.01448 & 1447 & 138.4 & 40.9 & 951.9 & 0.0331638 \\
\hline 3.4 & 203 & 2.4 & 800 & 0.81 & 0.09 & 1560 & 86.47 & 163.2 & 0.0008889 & 0.01458 & 1451 & 138.3 & 40.7 & 952 & 0.0334426 \\
\hline 7.9 & 298 & 2.4 & 800 & 0.85 & 0.14 & 1560 & 86.47 & 163.2 & 0.0008889 & 0.01458 & 1451 & 138.3 & 40.7 & 952 & 0.0334426 \\
\hline 8.3 & 299 & 2.4 & 800 & 0.84 & 0.14 & 1560 & 86.47 & 163.2 & 0.0008889 & 0.01458 & 1451 & 138.3 & 40.7 & 952 & 0.0334426 \\
\hline 12.2 & 399 & 2.4 & 800 & 0.85 & 0.13 & 1590 & 86.47 & 162.3 & 0.0008917 & 0.01428 & 1438 & 138.8 & 41.5 & 951.5 & 0.0322305 \\
\hline 12.1 & 399 & 2.4 & 800 & 0.85 & 0.12 & 1610 & 86.47 & 161.6 & 0.0008936 & 0.01408 & 1429 & 139.1 & 42 & 951.2 & 0.0314864 \\
\hline 12.3 & 401 & 2.4 & 800 & 0.84 & 0.12 & 1600 & 86.47 & 161.6 & 0.0008936 & 0.01408 & 1429 & 139.1 & 41.7 & 951.4 & 0.0319522 \\
\hline 1.7 & 122 & 5.1 & 800 & 0.82 & 0.14 & 1540 & 86.47 & 163.8 & 0.000887 & 0.01479 & 1460 & 137.9 & 40.2 & 952.3 & 0.0342799 \\
\hline 1.6 & 124 & 5.1 & 800 & 0.88 & 0.12 & 1570 & 86.47 & 162.9 & 0.0008898 & 0.01448 & 1447 & 138.4 & 40.9 & 951.9 & 0.0331638 \\
\hline 1.7 & 126 & 5.1 & 800 & 0.81 & 0.09 & 1560 & 86.47 & 163.2 & 0.0008889 & 0.01458 & 1451 & 138.3 & 40.7 & 952 & 0.0334426 \\
\hline 4.2 & 200 & 5.1 & 800 & 0.8 & 0.08 & 1580 & 86.47 & 162.5 & 0.0008908 & 0.01438 & 1442 & 138.6 & 41.2 & 951.7 & 0.032697 \\
\hline 4.3 & 201 & 5.1 & 800 & 0.81 & 0.09 & 1590 & 86.47 & 162.3 & 0.0008917 & 0.01428 & 1438 & 138.8 & 41.5 & 951.5 & 0.0322305 \\
\hline 4 & 202 & 5.1 & 800 & 0.85 & 0.11 & 1600 & 86.47 & 161.6 & 0.0008936 & 0.01408 & 1429 & 139.1 & 41.7 & 951.4 & 0.0319522 \\
\hline 7.8 & 295 & 5.1 & 800 & 0.83 & 0.11 & 1520 & $\begin{array}{l}80.47 \\
86.47\end{array}$ & 164.4 & 0.0008851 & 0.015 & 1469 & 137.6 & 39.6 & 952.6 & 0.0352089 \\
\hline 8.6 & 298 & 5.1 & 800 & 0.82 & 0.09 & 1510 & 86.47 & 164.8 & 0.0008841 & 0.01511 & 1474 & 137.4 & 39.4 & 952.8 & 0.0355869 \\
\hline 8.1 & 301 & 5.1 & 800 & 0.9 & 0.14 & 1530 & 86.47 & 164.2 & 0.000886 & 0.01489 & 1464 & 137.8 & 39.9 & 952.5 & 0.0347479 \\
\hline 7.9 & 304 & 5.1 & 800 & 0.84 & 0.1 & 1520 & 86.47 & 164.4 & 0.0008851 & 0.015 & 1469 & 137.6 & 39.6 & 952.6 & 0.0352089 \\
\hline 13.5 & 403 & 5.1 & 800 & 0.86 & 0.11 & 1520 & 86.47 & 164.4 & 0.0008851 & 0.015 & 1469 & 137.6 & 39.6 & 952.6 & 0.0352089 \\
\hline 12.6 & 405 & 5.1 & 800 & 0.86 & 0.09 & 1560 & 86.47 & 163.2 & 0.0008889 & 0.01458 & 1451 & 138.3 & 40.7 & 952 & 0.0334426 \\
\hline 13.1 & 413 & 5.1 & 800 & 0.84 & 0.1 & 1560 & 86.47 & 163.2 & 0.0008889 & 0.01458 & 1451 & 138.3 & 40.7 & 952 & 0.0334426 \\
\hline
\end{tabular}

\section{R22 with SUS300 mineral oil}

$\begin{array}{lllllll}1.4 & 120 & 0.6 & 800 & 0.88 & 0.05 & 1590 \\ 1.3 & 120 & 0.6 & 800 & 0.87 & 0.06 & 1650 \\ 1.2 & 125 & 0.6 & 800 & 0.84 & 0.04 & 1630 \\ 3.6 & 193 & 0.6 & 800 & 0.86 & 0.12 & 1540 \\ 3.4 & 194 & 0.6 & 800 & 0.86 & 0.09 & 1540 \\ 3.6 & 198 & 0.6 & 800 & 0.88 & 0.1 & 1550 \\ 8 & 297 & 0.6 & 800 & 0.87 & 0.14 & 1600 \\ 7.9 & 298 & 0.6 & 800 & 0.87 & 0.14 & 1600 \\ 7.7 & 298 & 0.6 & 800 & 0.87 & 0.13 & 1610 \\ 11.8 & 399 & 0.6 & 800 & 0.88 & 0.13 & 1610 \\ 12.4 & 400 & 0.6 & 800 & 0.88 & 0.14 & 1600 \\ 11.5 & 403 & 0.6 & 800 & 0.87 & 0.15 & 1590 \\ 1.7 & 128 & 1.3 & 800 & 0.87 & 0.06 & 1630 \\ 1.6 & 128 & 1.3 & 800 & 0.87 & 0.04 & 1600 \\ 1.7 & 131 & 1.3 & 800 & 0.85 & 0.04 & 1590 \\ 3.9 & 200 & 1.3 & 800 & 0.91 & 0.16 & 1590 \\ 3.9 & 203 & 1.3 & 800 & 0.89 & 0.17 & 1580 \\ 3.7 & 204 & 1.3 & 800 & 0.89 & 0.18 & 1560 \\ 8.3 & 296 & 1.3 & 800 & 0.91 & 0.16 & 1550 \\ 8.1 & 300 & 1.3 & 800 & 0.89 & 0.15 & 1550 \\ 8.2 & 300 & 1.3 & 800 & 0.9 & 0.14 & 1550 \\ 13.2 & 398 & 1.3 & 800 & 0.85 & 0.15 & 1520 \\ 12.8 & 399 & 1.3 & 800 & 0.85 & 0.14 & 1530 \\ 13.1 & 404 & 1.3 & 800 & 0.85 & 0.14 & 1520 \\ 1.6 & 128 & 2.6 & 800 & 0.84 & 0.07 & 1590 \\ 1.8 & 129 & 2.6 & 800 & 0.88 & 0.17 & 1540 \\ 4.2 & 198 & 2.6 & 800 & 0.89 & 0.13 & 1500 \\ 3.9 & 199 & 2.6 & 800 & 0.9 & 0.13 & 1500 \\ 4.3 & 203 & 2.6 & 800 & 0.89 & 0.15 & 1510 \\ 8.6 & 293 & 2.6 & 800 & 0.86 & 0.11 & 1540 \\ 8.3 & 294 & 2.6 & 800 & 0.87 & 0.12 & 1540 \\ 8.7 & 297 & 2.6 & 800 & 0.88 & 0.1 & 1540 \\ 13.7 & 395 & 2.6 & 800 & 0.85 & 0.13 & 1550 \\ 13.5 & 399 & 2.6 & 800 & 0.86 & 0.12 & 1580 \\ 13.9 & 400 & 2.6 & 800 & 0.86 & 0.13 & 1570 \\ 1.8 & 120 & 5 & 800 & 0.85 & 0.12 & 1530 \\ 2 & 124 & 5 & 800 & 0.82 & 0.1 & 1550 \\ 4.5 & 208 & 5 & 800 & 0.84 & 0.09 & 1580 \\ 4.2 & 208 & 5 & 800 & 0.84 & 0.09 & 1560 \\ 4.7 & 211 & 5 & 800 & 0.83 & 0.11 & 1560 \\ 9.7 & 294 & 5 & 800 & 0.84 & 0.17 & 1500 \\ 9.3 & 297 & 5 & 800 & 0.88 & 0.15 & 1520 \\ 9.6 & 301 & 5 & 800 & 0.85 & 0.13 & 1520 \\ 13.5 & 394 & 5 & 800 & 0.83 & 0.06 & 1550 \\ 13.7 & 398 & 5 & 800 & 0.85 & 0.06 & 1570 \\ 14.5 & 401 & 5 & 800 & 0.85 & 0.1 & 1570 \\ & & & & & & \\ & & 5 & & & & \end{array}$

\begin{tabular}{ll}
86.47 & 162. \\
86.47 & 160. \\
86.47 & 161 \\
86.47 & 163. \\
86.47 & 163. \\
86.47 & 163. \\
86.47 & 161. \\
86.47 & 166. \\
86.47 & 161. \\
86.47 & 161. \\
86.47 & 161. \\
86.47 & 162. \\
86.47 & 161 \\
86.47 & 161. \\
86.47 & 162. \\
86.47 & 162. \\
86.47 & 162. \\
86.47 & 163. \\
86.47 & 163. \\
86.47 & 163. \\
86.47 & 163. \\
86.47 & 164.4 \\
86.47 & 164. \\
86.47 & 164. \\
86.47 & 162. \\
86.47 & 163. \\
86.47 & 165 \\
86.47 & 165 \\
86.47 & 164. \\
86.47 & 163. \\
86.47 & 163. \\
86.47 & 163. \\
86.47 & 163. \\
86.47 & 162. \\
86.47 & 162. \\
86.47 & 164. \\
86.47 & 163. \\
86.47 & 162. \\
86.47 & 163. \\
86.47 & 163. \\
86.47 & 165 \\
86.47 & 164.4 \\
86.47 & 164. \\
86.47 & 163. \\
86.47 & 162. \\
86.47 & 162. \\
& \\
\hline
\end{tabular}

$\begin{array}{lll}0.0008917 & 0.01428 & 1438\end{array}$

138.8

$\begin{array}{lll}41.5 & 953 & 0.0500423 \\ 43 & 952.1 & 0.0465938 \\ 42.5 & 952.4 & 0.0477117 \\ 40.2 & 955.8 & 0.0531285 \\ 40.2 & 953.8 & 0.0531285 \\ 40.4 & 953.7 & 0.0526626 \\ 41.7 & 952.9 & 0.0495778 \\ 41.7 & 952.9 & 0.0495778 \\ 42 & 952.7 & 0.0488309 \\ 42 & 952.7 & 0.0488309 \\ 41.7 & 955.9 & 0.0495778 \\ 41.5 & 953 & 0.0500423 \\ 42.5 & 952.4 & 0.0477117 \\ 41.7 & 952.9 & 0.0495778 \\ 41.5 & 953 & 0.0500423 \\ 41.5 & 953 & 0.0500423 \\ 41.2 & 953.2 & 0.0506993 \\ 40.7 & 953.5 & 0.0519132 \\ 40.4 & 953.7 & 0.052626 \\ 40.4 & 953.7 & 0.0526626 \\ 40.4 & 953.7 & 0.0526626 \\ 39.6 & 954.1 & 0.0546184 \\ 39.9 & 954 & 0.0538789 \\ 39.6 & 954.1 & 0.0546184 \\ 41.5 & 953 & 0.0500423 \\ 40.2 & 953.8 & 0.0531285 \\ 39.1 & 954.4 & 0.055928 \\ 39.1 & 954.4 & 0.059928 \\ 39.4 & 954.3 & 0.0551877 \\ 40.2 & 953.8 & 0.0531285 \\ 40.2 & 953.8 & 0.0531285 \\ 40.2 & 953.8 & 0.0531285 \\ 40.4 & 953.7 & 0.0526626 \\ 41.2 & 955.2 & 0.0506993 \\ 40.9 & 953.4 & 0.0514478 \\ 39.9 & 954 & 0.0538789 \\ 40.4 & 953.7 & 0.0526626 \\ 41.2 & 953.2 & 0.0506993 \\ 40.7 & 953.5 & 0.0519132 \\ 40.7 & 953.5 & 0.0519132 \\ 39.1 & 955.4 & 0.055928 \\ 39.6 & 954.1 & 0.0546184 \\ 39.6 & 954.1 & 0.0546184 \\ 40.4 & 953.7 & 0.0526626 \\ 40.9 & 953.4 & 0.0514478 \\ 40.9 & 953.4 & 0.0514478 \\ & & \end{array}$

Micro-fin 3/8 inch, evaporation

\section{R12 with SUS150 naphthenic oil}
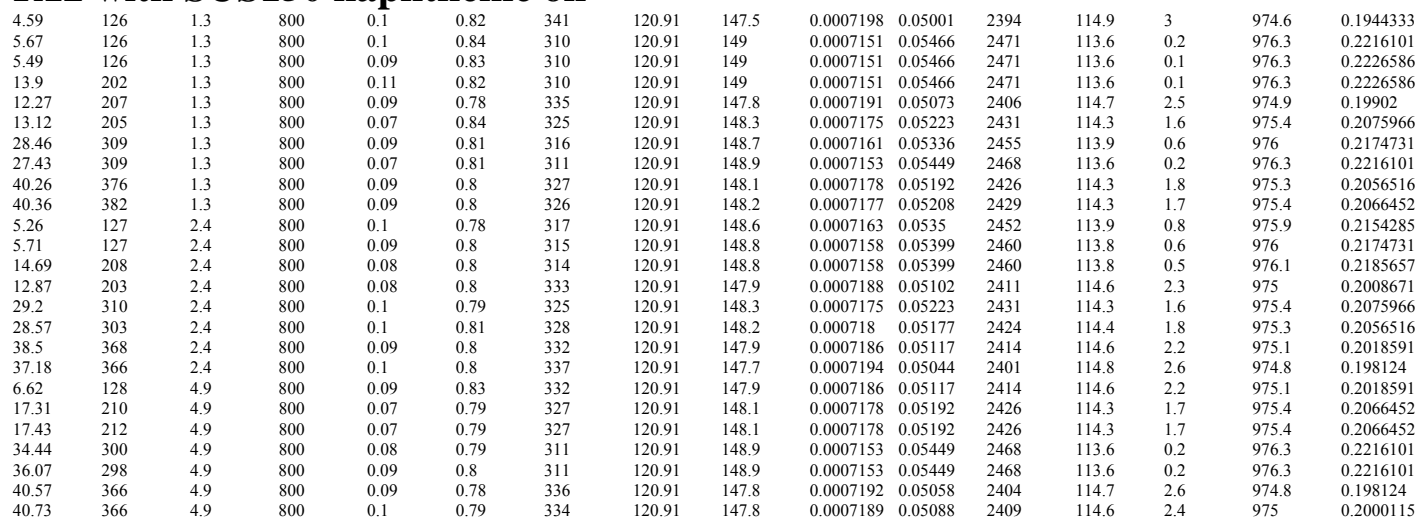

R12 with SUS300 naphthenic oil
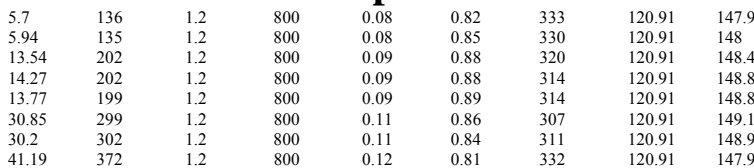

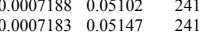

$\begin{array}{lll}0.000717 & 0.0527 & 2439 \\ 0.0007158 & 0.05399 & 2460\end{array}$

$\begin{array}{lll}0.0007158 & 0.05399 & 2460\end{array}$

$\begin{array}{lll}0.0007147 & 0.05517 & 2479\end{array}$

$\begin{array}{llll}114.6 & 2.3 & 976.5 & 0.3116209 \\ 114.5 & 2 & 976.7 & 0.3160413 \\ 114.1 & 1.1 & 977.2 & 0.3295428 \\ 113.8 & 0.5 & 977.6 & 0.3389874 \\ 113.8 & 0.5 & 977.6 & 0.3389874 \\ 113.5 & -0.2 & 978 & 0.3501688 \\ 113.6 & 0.2 & 977.8 & 0.3437154 \\ 114.6 & 2.2 & 976.6 & 0.3131153\end{array}$ 


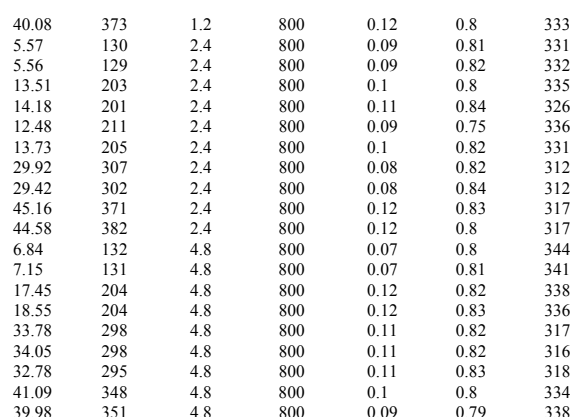

$\begin{array}{lllllllll}120.91 & 147.9 & 0.0007188 & 0.05102 & 2411 & 114.6 & 2.3 & 976.5 & 0.3116209 \\ 120.91 & 148 & 0.0007185 & 0.055132 & 2416 & 114.5 & 2.1 & 976.6 & 0.3145459 \\ 120.91 & 147.9 & 0.0007186 & 0.05117 & 2414 & 114.6 & 2.2 & 976.6 & 0.3131153 \\ 120.91 & 147.8 & 0.0007191 & 0.05073 & 2406 & 114.7 & 2.4 & 976.5 & 0.3101905 \\ 120.91 & 148.1 & 0.0007177 & 0.05208 & 2429 & 114.3 & 1.9 & 976.8 & 0.3175372 \\ 120.91 & 147.8 & 0.0007192 & 0.05058 & 2404 & 114.7 & 2.6 & 976.3 & 0.3072993 \\ 120.91 & 148 & 0.0007185 & 0.05132 & 2416 & 114.5 & 2 & 976.7 & 0.3160413 \\ 120.91 & 148.8 & 0.0007155 & 0.05432 & 2465 & 113.7 & 0.3 & 977.7 & 0.3421157 \\ 120.91 & 148.8 & 0.0007155 & 0.05432 & 2465 & 113.7 & 0.3 & 977.7 & 0.3421157 \\ 120.91 & 148.6 & 0.0007163 & 0.0535 & 2452 & 113.9 & 0.8 & 977.4 & 0.3342633 \\ 120.91 & 148.6 & 0.0007163 & 0.0535 & 2452 & 113.9 & 0.8 & 977.4 & 0.3342633 \\ 120.91 & 147.3 & 0.0007202 & 0.04972 & 2389 & 115 & 3.2 & 976 & 0.2988231 \\ 120.91 & 147.5 & 0.0007198 & 0.05001 & 2394 & 114.9 & 3 & 976.1 & 0.3016474 \\ 120.91 & 147.6 & 0.0007195 & 0.0503 & 2399 & 114.8 & 2.7 & 976.3 & 0.3058696 \\ 120.91 & 147.8 & 0.0007192 & 0.05058 & 2404 & 114.7 & 2.6 & 976.3 & 0.3072993 \\ 120.91 & 148.6 & 0.0007163 & 0.0535 & 2452 & 113.9 & 0.8 & 977.4 & 0.3342633 \\ 120.91 & 148.7 & 0.0007161 & 0.05336 & 2455 & 113.9 & 0.6 & 977.5 & 0.3373893 \\ 120.91 & 148.6 & 0.0007164 & 0.05334 & 2449 & 113.9 & 0.9 & 977.4 & 0.3327348 \\ 120.91 & 147.8 & 0.0007189 & 0.05088 & 2409 & 114.6 & 2.4 & 976.5 & 0.3101905 \\ 120.91 & 147.6 & 0.0007195 & 0.0503 & 2399 & 114.8 & 2.7 & 976.3 & 0.3058696\end{array}$

R134a with SUS169 ester-m oil

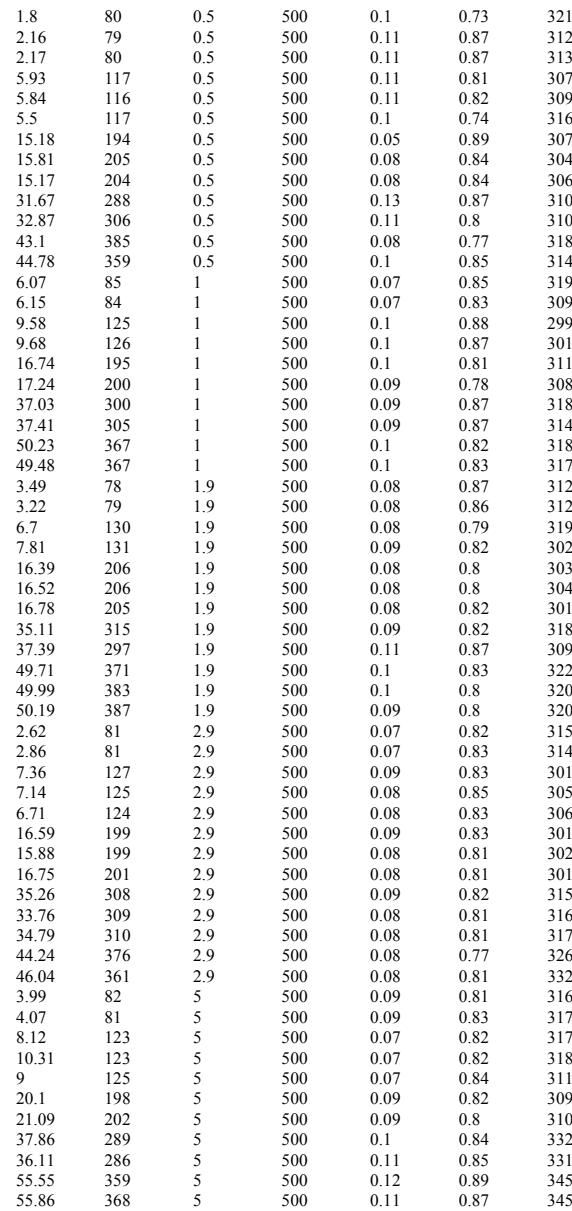

102.03

$0.0007781 \quad 0.06343$

$\begin{array}{llll}110.3 & 2.5 & 975.1 & 0.213364 \\ 110 & 1.8 & 975.5 & 0.2203906\end{array}$

$\begin{array}{lllllllll}102.03 & 197.3 & 0.0007765 & 0.0652 & 2813 & 110 & 1.8 & 975.5 & 0.2203906\end{array}$ $\begin{array}{lllllllll}102.03 & 197.3 & 0.0007767 & 0.065 & 2810 & 110 & 1.8 & 975.5 & 0.2203906 \\ 102.03 & 197.6 & 0.0007756 & 0.06623 & 2830 & 109.8 & 1.3 & 975 & 0.225728\end{array}$ $\begin{array}{lllll}102.03 & 197.6 & 0.0007756 & 0.06623 & 2830 \\ 102.03 & 197 & 0.000776 & 0.06581 & 2823\end{array}$ $\begin{array}{lllll}102.03 & 197 & 0.0007772 & 0.06581 & 2823 \\ 0.0644 & 2800\end{array}$ $\begin{array}{lllll}102.03 & 197.6 & 0.0007756 & 0.06623 & 2830\end{array}$ $\begin{array}{lllll}102.03 & 197.9 & 0.0007751 & 0.06686 & 2840 \\ 102.03 & 197.7 & 0.0007754 & 0.06644 & 2833\end{array}$ $\begin{array}{lllll}102.03 & 197.4-0.0007761 & 0.06561 & 2820\end{array}$ $\begin{array}{lllll}102.03 & 197.4 & 0.0007761 & 0.06561 & 2820\end{array}$ $\begin{array}{lllll}102.03 & 196.9 & 0.0007776 & 0.06401 & 2794\end{array}$ $\begin{array}{llllll}102.03 & 197.2 & 0.0007768 & 0.0648 & 2807 \\ 102.03 & 196.9 & 0.0007777 & 0.06382 & 2791\end{array}$ $\begin{array}{lll}0.000776 & 0.06581 & 2823\end{array}$ $102.03 \quad 19.3$

R134a with SUS369 ester-m oil

$\begin{array}{lllllll}4.49 & 87 & 0.6 & 500 & 0.09 & 0.85 & 312 \\ 4.63 & 87 & 0.6 & 500 & 0.1 & 0.83 & 308 \\ .51 & 127 & 0.6 & 500 & 0.1 & 0.82 & 309 \\ 7.27 & 127 & 0.6 & 500 & 0.11 & 0.83 & 310 \\ 14.77 & 201 & 0.6 & 500 & 0.07 & 0.82 & 292 \\ 15.2 & 198 & 0.6 & 500 & 0.07 & 0.82 & 292 \\ 30.05 & 299 & 0.6 & 500 & 0.09 & 0.81 & 310 \\ 30.53 & 298 & 0.6 & 500 & 0.09 & 0.81 & 309 \\ 32.07 & 297 & 0.6 & 500 & 0.09 & 0.84 & 304 \\ 42.85 & 356 & 0.6 & 500 & 0.1 & 0.85 & 324 \\ 44.41 & 364 & 0.6 & 500 & 0.09 & 0.84 & 317 \\ 2.62 & 86 & 1.1 & 500 & 0.09 & 0.8 & 313 \\ .72 & 85 & 1.1 & 500 & 0.09 & 0.8 & 314 \\ 5.37 & 126 & 1.1 & 500 & 0.08 & 0.83 & 314 \\ 5.98 & 127 & 1.1 & 500 & 0.09 & 0.84 & 313 \\ 5.93 & 127 & 1.1 & 500 & 0.08 & 0.84 & 315 \\ 15.06 & 198 & 1.1 & 500 & 0.07 & 0.81 & 293 \\ 14.76 & 196 & 1.1 & 500 & 0.07 & 0.81 & 292 \\ 33.13 & 301 & 1.1 & 500 & 0.12 & 0.82 & 325 \\ 32.92 & 302 & 1.1 & 500 & 0.12 & 0.83 & 330 \\ 44.2 & 368 & 1.1 & 500 & 0.11 & 0.79 & 334 \\ 44.52 & 365 & 1.1 & 500 & 0.11 & 0.82 & 340 \\ 3.25 & 87 & 2.4 & 500 & 0.1 & 0.8 & 315 \\ 3.41 & 87 & 2.4 & 500 & 0.1 & 0.8 & 318 \\ 3.37 & 87 & 2.4 & 500 & 0.1 & 0.81 & 318 \\ 6.77 & 126 & 2.4 & 500 & 0.1 & 0.82 & 304 \\ & & & & & & \end{array}$

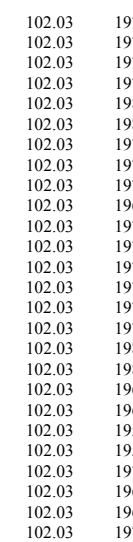

$\begin{array}{lll}0.0007765 & 0.0652 & 2813 \\ 0.0007758 & 0.06602 & 2826\end{array}$ $\begin{array}{lll}0.0007758 & 0.06602 & 2826 \\ 0.000776 & 0.06581 & 2823\end{array}$ $\begin{array}{lll}0.0007761 & 0.06561 & 2820\end{array}$ $\begin{array}{lll}0.0007739 & 0.06951 & 288 \\ 0.0007739 & 0.06951 & 288\end{array}$ $\begin{array}{lll}0.0007761 & 0.06561 & 2820\end{array}$ $\begin{array}{lll}0.000776 & 0.06581 & 2823\end{array}$ $\begin{array}{lll}0.0007751 & 0.06686 & 2840 \\ 0.0007786 & 0.06287 & 2775\end{array}$ $\begin{array}{lll}0.0007786 & 0.06287 & 2775 \\ 0.0007774 & 0.06421 & 2797\end{array}$ $\begin{array}{lll}0.0007774 & 0.0642\end{array}$ $\begin{array}{lll}0.0007767 & 0.065 & 2810 \\ 0.0007768 & 0.0648 & 2807\end{array}$ $\begin{array}{lll}0.0007768 & 0.0648 & 2807 \\ 0.0007767 & 0.065 & 2810\end{array}$ $\begin{array}{lll}0.0007767 & 0.065 & 2810 \\ 0.000777 & 0.0646 & 2803\end{array}$ $\begin{array}{lll}0.000777 & 0.0646 & 2803 \\ 0.0007731 & 0.06928 & 2878\end{array}$ $\begin{array}{lll}0.0007731 & 0.06928 & 2878 \\ 0.0007739 & 0.06951 & 2881\end{array}$ $\begin{array}{lll}0.0007788 & 0.06268 & 2772 \\ 0.0007797 & 0.06176 & 2756\end{array}$ $\begin{array}{lll}0.0007803 & 0.06104 & 2744\end{array}$ $\begin{array}{lll}0.0007814 & 0.06 & 2726 \\ 0.000777 & 0.0646 & 2803 \\ 0.007776 & 0.0640 & 2794\end{array}$ $\begin{array}{lll}0.00007776 & 0.0646 & 2801 \\ 0.0007776 & 0.06401 & 2794\end{array}$ $\begin{array}{lll}0.0007776 & 0.06401 & 2794 \\ 0.0007751 & 0.06686 & 2840\end{array}$

$\begin{array}{llll}110 & 1.8 & 977.5 & 0.3927131 \\ 109.8 & 1.4 & 977.8 & 0.4002205 \\ 109.9 & 1.5 & 977.7 & 0.3983224 \\ 109.9 & 1.6 & 977.6 & 0.396425 \\ 109.2 & 0.2 & 978.5 & 0.4231983 \\ 109.2 & 0.1 & 978.5 & 0.4251132 \\ 109.9 & 1.6 & 977.6 & 0.396425 \\ 109.9 & 1.5 & 977.7 & 0.3983224 \\ 109.7 & 1.1 & 977.9 & 0.4057532 \\ 110.5 & 2.8 & 976.9 & 0.3748623 \\ 110.2 & 2.2 & 977.3 & 0.3854845 \\ 110 & 1.8 & 977.5 & 0.3927131 \\ 110.1 & 1.9 & 977.5 & 0.3909931 \\ 110.1 & 1.9 & 977.5 & 0.3909931 \\ 110 & 1.8 & 977.5 & 0.3927131 \\ 110.1 & 2 & 977.4 & 0.3890981 \\ 109.2 & 0.3 & 978.4 & 0.4211973 \\ 109.2 & 0.1 & 978.5 & 0.4251132 \\ 110.5 & 2.8 & 976.9 & 0.3748623 \\ 110.7 & 3.3 & 976.6 & 0.3661437 \\ 110.8 & 3.6 & 976.4 & 0.3611316 \\ 11.1 & 4.1 & 976.1 & 0.3527159 \\ 110.1 & 2 & 977.4 & 0.3890981 \\ 110.2 & 2.3 & 977.2 & 0.3836868 \\ 110.2 & 2.3 & 977.2 & 0.3836868 \\ 109.7 & 1 & 978 & 0.4076535\end{array}$



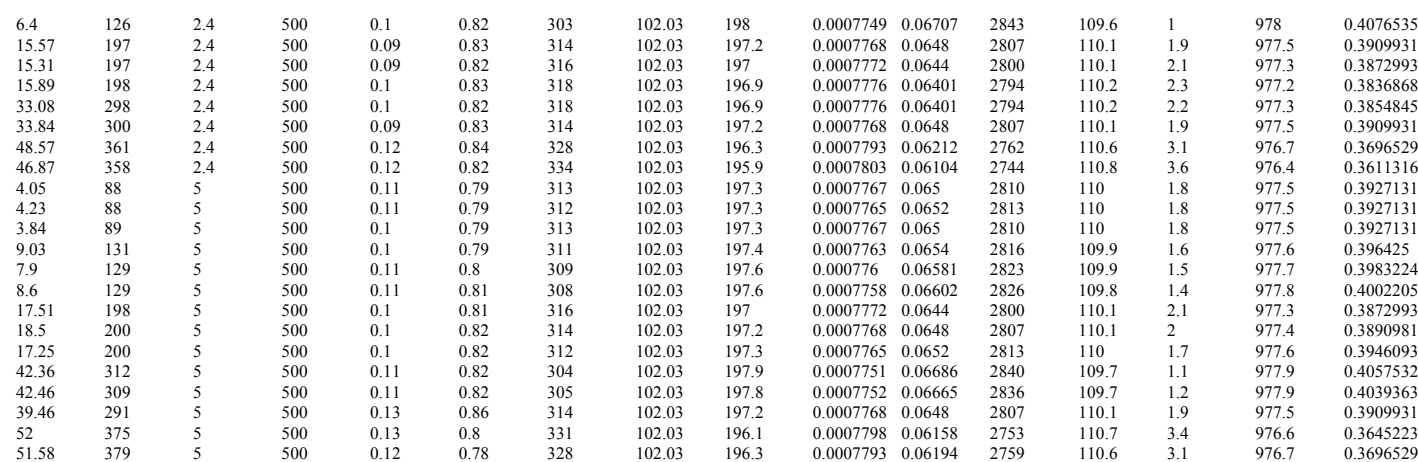

R134a with SUS150 ester-b oil

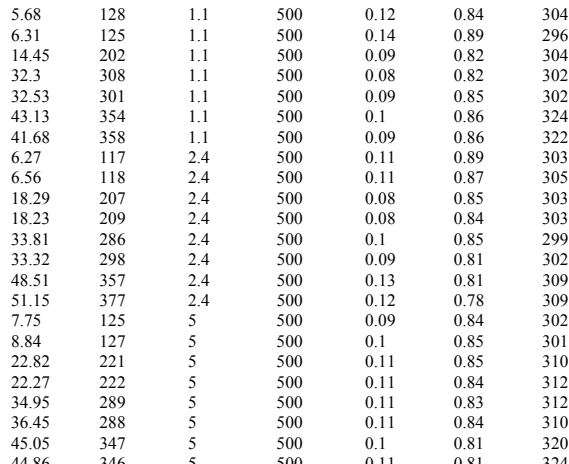

$\begin{array}{lllllllll}102.03 & 197.9 & 0.0007751 & 0.06686 & 2840 & 109.7 & 1.1 & 975.7 & 0.2124843 \\ 102.03 & 198.5 & 0.0007736 & 0.0686 & 2867 & 109.3 & 0.4 & 976.2 & 0.2195635 \\ 102.03 & 197.9 & 0.0007751 & 0.06686 & 2840 & 109.7 & 1 & 975.8 & 0.21348 \\ 102.03 & 198 & 0.0077477 & 0.06728 & 2847 & 109.6 & 0.9 & 975.9 & 0.2144762 \\ 102.03 & 198 & 0.0007747 & 0.06728 & 2847 & 109.6 & 0.9 & 975.9 & 0.2144762 \\ 102.03 & 196.6 & 0.0007786 & 0.06287 & 2775 & 110.5 & 2.8 & 974.7 & 0.1962783 \\ 102.03 & 196.8 & 0.0007779 & 0.06363 & 2787 & 110.3 & 2.6 & 974.8 & 0.198124 \\ 102.03 & 198 & 0.0007749 & 0.06707 & 2843 & 109.6 & 1 & 975.8 & 0.21348 \\ 102.03 & 197.8 & 0.0007752 & 0.06665 & 2836 & 109.7 & 1.1 & 975.7 & 0.2124843 \\ 102.03 & 198 & 0.0007749 & 0.06707 & 2843 & 109.6 & 1 & 975.8 & 0.21348 \\ 102.03 & 198 & 0.0007749 & 0.06707 & 2843 & 109.6 & 1 & 975.8 & 0.21348 \\ 102.03 & 198.3 & 0.0007742 & 0.06794 & 2857 & 109.4 & 0.7 & 976 & 0.2165205 \\ 102.03 & 198 & 0.0007747 & 0.06728 & 2847 & 109.6 & 0.9 & 975.9 & 0.2144762 \\ 102.03 & 197.6 & 0.000776 & 0.06551 & 2823 & 109.9 & 1.5 & 975.5 & 0.2085908 \\ 102.03 & 197.6 & 0.00776 & 0.06581 & 2823 & 109.9 & 1.5 & 975.5 & 0.2085908 \\ 102.03 & 198 & 0.0007747 & 0.06728 & 2847 & 109.6 & 0.9 & 975.9 & 0.2144762 \\ 102.03 & 198.1 & 0.0007745 & 0.0675 & 2850 & 109.5 & 0.8 & 975.9 & 0.2154285 \\ 102.03 & 197.4 & 0.0007761 & 0.06561 & 2820 & 109.9 & 1.6 & 975.4 & 0.2075966 \\ 102.03 & 197.3 & 0.0007765 & 0.0652 & 2813 & 110 & 1.8 & 975.3 & 0.2056516 \\ 102.03 & 197.3 & 0.0007765 & 0.0652 & 2813 & 110 & 1.7 & 975.4 & 0.2066452 \\ 102.03 & 197.4 & 0.0077761 & 0.06561 & 2820 & 109.9 & 1.6 & 975.4 & 0.2075966 \\ 102.03 & 196.8 & 0.0007779 & 0.06363 & 2787 & 110.3 & 2.5 & 974.9 & 0.19902 \\ 102.03 & 196.6 & 0.0007786 & 0.06287 & 2775 & 110.5 & 2.8 & 974.7 & 0.1962783\end{array}$

\section{R22 with SUS150 mineral oil}

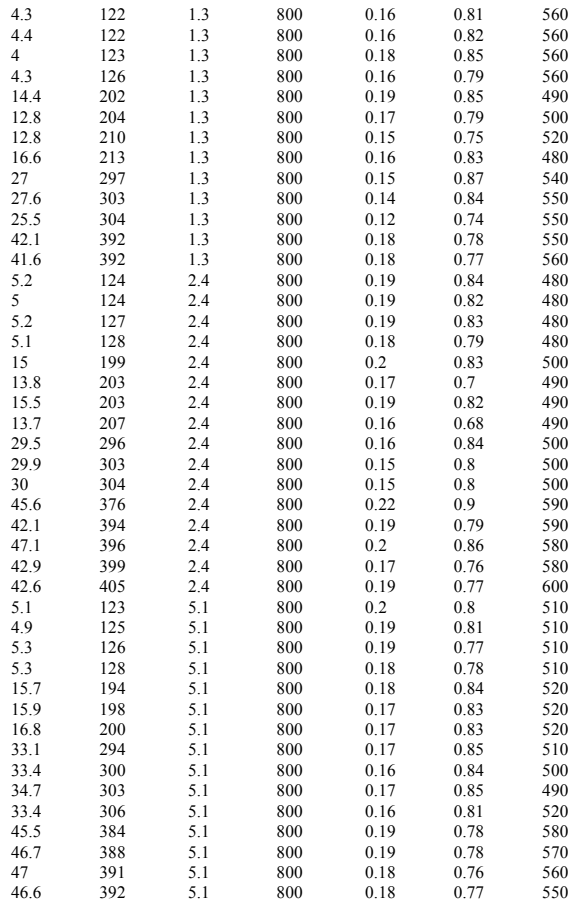

\begin{tabular}{|c|c|c|c|c|c|c|c|c|}
\hline 86.47 & 199.5 & 0.0007872 & 0.04166 & 2118 & 118.4 & 3.7 & 974.2 & 0.1881997 \\
\hline 86.47 & 199.5 & 0.0007872 & 0.04166 & 2118 & 118.4 & 3.7 & 974.2 & 0.1881997 \\
\hline 86.47 & 199.5 & 0.0007872 & 0.04166 & 2118 & 118.4 & 3.7 & 974.2 & 0.1881997 \\
\hline 86.47 & 199.5 & 0.0007872 & 0.04166 & 2118 & 118.4 & 3.7 & 974.2 & 0.1881997 \\
\hline 86.47 & 202.8 & 0.0007786 & 0.04745 & 2204 & 116.4 & -0.5 & 976.7 & 0.2289463 \\
\hline 86.47 & 202.3 & 0.0007798 & 0.04653 & 2191 & 116.7 & 0.1 & 976.3 & 0.2226586 \\
\hline 86.47 & 201.4 & 0.0007823 & 0.04478 & 2166 & 117.3 & 1.3 & 975.6 & 0.2105371 \\
\hline 86.47 & 203.3 & 0.0007773 & 0.04841 & 2218 & 116.1 & -1.1 & 978.6 & 0.3650592 \\
\hline 86.47 & 200.4 & 0.0007848 & 0.04316 & 2141 & 117.8 & 2.5 & 974.9 & 0.19902 \\
\hline 86.47 & 200 & 0.000786 & 0.0424 & 2130 & 118.1 & 3.1 & 974.5 & 0.1935387 \\
\hline 86.47 & 200 & 0.000786 & 0.0424 & 2130 & 118.1 & 3.1 & 974.5 & 0.1935387 \\
\hline 86.47 & 200 & 0.000786 & 0.0424 & 2130 & 118.1 & 3.1 & 974.5 & 0.1935387 \\
\hline 86.47 & 199.5 & 0.0007872 & 0.04166 & 2118 & 118.4 & 3.7 & 974.2 & 0.1881997 \\
\hline 86.47 & 203.3 & 0.0007773 & 0.04841 & 2218 & 116.1 & -1.1 & 978.6 & 0.3650592 \\
\hline 86.47 & 203.3 & 0.0007773 & 0.04841 & 2218 & 116.1 & -1.1 & 978.6 & 0.3650592 \\
\hline 86.47 & 203.3 & 0.0007773 & 0.04841 & 2218 & 116.1 & -1.1 & 978.6 & 0.3650592 \\
\hline 86.47 & 203.3 & 0.0007773 & 0.04841 & 2218 & 116.1 & -1.1 & 978.6 & 0.3650592 \\
\hline 86.47 & 202.3 & 0.0007798 & 0.04653 & 2191 & 116.7 & 0.1 & 976.3 & 0.2226586 \\
\hline 86.47 & 202.8 & 0.0007786 & 0.04745 & 2204 & 116.4 & -0.5 & 976.7 & 0.2289463 \\
\hline 86.47 & 202.8 & 0.0007786 & 0.04745 & 2204 & 116.4 & -0.5 & 976.7 & 0.2289463 \\
\hline 86.47 & 202.8 & 0.0007786 & 0.04745 & 2204 & 116.4 & -0.5 & 976.7 & 0.2289463 \\
\hline 86.47 & 202.3 & 0.0007798 & 0.04653 & 2191 & 116.7 & 0.1 & 976.3 & 0.2226586 \\
\hline 86.47 & 202.3 & 0.0007798 & 0.04653 & 2191 & 116.7 & 0.1 & 976.3 & 0.2226586 \\
\hline 86.47 & 202.3 & 0.0007798 & 0.04653 & 2191 & 116.7 & 0.1 & 976.3 & 0.2226586 \\
\hline 86.47 & 198.2 & 0.0007908 & 0.03958 & 2085 & 119.2 & 5.3 & 973.2 & 0.1746486 \\
\hline 86.47 & 198.2 & 0.0007908 & 0.03958 & 2085 & 119.2 & 5.3 & 973.2 & 0.1746486 \\
\hline 86.47 & 198.8 & 0.0007896 & 0.04025 & 2095 & 118.9 & 4.8 & 973.5 & 0.1787366 \\
\hline 86.47 & 198.8 & 0.0007896 & 0.04025 & 2095 & 118.9 & 4.8 & 973.5 & 0.1787366 \\
\hline 86.47 & 197.6 & 0.000792 & 0.03893 & 2074 & 119.4 & 5.9 & 972.9 & 0.1699029 \\
\hline 86.47 & 201.9 & 0.0007811 & 0.04564 & 2178 & 117 & 0.7 & 976 & 0.2165205 \\
\hline 86.47 & 201.9 & 0.0007811 & 0.04564 & 2178 & 117 & 0.7 & 976 & 0.2165205 \\
\hline 86.47 & 201.9 & 0.0007811 & 0.04564 & 2178 & 117 & 0.7 & 976 & 0.2165205 \\
\hline 86.47 & 201.9 & 0.0007811 & 0.04564 & 2178 & 117 & 0.7 & 976 & 0.2165205 \\
\hline 86.47 & 201.4 & 0.0007823 & 0.04478 & 2166 & 117.3 & 1.3 & 975.6 & 0.2105371 \\
\hline 86.47 & 201.4 & 0.0007823 & 0.04478 & 2166 & 117.3 & 1.3 & 975.6 & 0.2105371 \\
\hline 86.47 & 201.4 & 0.0007823 & 0.04478 & 2166 & 117.3 & 1.3 & 975.6 & 0.2105371 \\
\hline 86.47 & 201.9 & 0.0007811 & 0.04564 & 2178 & 117 & 0.7 & 976 & 0.2165205 \\
\hline 86.47 & 202.3 & 0.0007798 & 0.04653 & 2191 & 116.7 & 0.1 & 976.3 & 0.2226586 \\
\hline 86.47 & 202.8 & 0.0007786 & 0.04745 & 2204 & 116.4 & -0.5 & 976.7 & 0.2289463 \\
\hline 86.47 & 201.4 & 0.0007823 & 0.04478 & 2166 & 117.3 & 1.3 & 975.6 & 0.2105371 \\
\hline 86.47 & 198.8 & 0.0007896 & 0.04025 & 2095 & 118.9 & 4.8 & 973.5 & 0.1787366 \\
\hline 86.47 & 199 & 0.0007884 & 0.04094 & 2107 & 118.6 & 4.2 & 973.9 & 0.1839105 \\
\hline 86.47 & 199.5 & 0.0007872 & 0.04166 & 2118 & 118.4 & 3.7 & 974.2 & 0.1881997 \\
\hline 86.47 & 200 & 0.000786 & 0.0424 & 2130 & 118.1 & 3.1 & 974.5 & 0.1935387 \\
\hline
\end{tabular}

\section{R22 with SUS300 mineral oil}

\begin{tabular}{|c|c|c|c|c|c|}
\hline 6.2 & 119 & 0.6 & 800 & 0.11 & 0.93 \\
\hline 5.1 & 124 & 0.6 & 800 & 0.1 & 0.81 \\
\hline 5 & 129 & 0.6 & 800 & 0.11 & 0.79 \\
\hline 14.9 & 203 & 0.6 & 800 & 0.17 & 0.86 \\
\hline 14.6 & 204 & 0.6 & 800 & 0.17 & 0.81 \\
\hline 14.6 & 205 & 0.6 & 800 & 0.16 & 0.85 \\
\hline 27.6 & 292 & 0.6 & 800 & 0.17 & 0.78 \\
\hline 28.2 & 294 & 0.6 & 800 & 0.13 & 0.79 \\
\hline 28.7 & 300 & 0.6 & 800 & 0.13 & 0.77 \\
\hline 30.3 & 304 & 0.6 & 800 & 0.15 & 0.82 \\
\hline 44.5 & 353 & 0.6 & 800 & 0.14 & 0.89 \\
\hline 46 & 385 & 0.6 & 800 & 0.18 & 0.83 \\
\hline 44.6 & 391 & 0.6 & 800 & 0.19 & 0.78 \\
\hline & & & & & \\
\hline
\end{tabular}

$\begin{array}{lllllllll}86.47 & 203.8 & 0.000776 & 0.04941 & 2231 & 115.8 & -1.7 & 979 & 0.3749421 \\ 86.47 & 203.3 & 0.0007773 & 0.04481 & 2218 & 116.1 & -1.1 & 978.6 & 0.3650592 \\ 86.47 & 204.3 & 0.0007747 & 0.05046 & 2245 & 115.5 & -2.4 & 979.5 & 0.3865505 \\ 86.47 & 201.4 & 0.0007823 & 0.04478 & 2166 & 117.3 & 1.3 & 977.1 & 0.3265157 \\ 86.47 & 201.4 & 0.0007823 & 0.04478 & 2166 & 117.3 & 1.3 & 977.1 & 0.3265157 \\ 86.47 & 201.4 & 0.0007823 & 0.04478 & 2166 & 117.3 & 1.3 & 977.1 & 0.32655157 \\ 86.47 & 201.4 & 0.0007823 & 0.04478 & 2166 & 117.3 & 1.3 & 977.1 & 0.3265157 \\ 86.47 & 202.3 & 0.0007798 & 0.04653 & 2191 & 116.7 & 0.1 & 977.8 & 0.3453407 \\ 86.47 & 202.3 & 0.0007798 & 0.04653 & 2191 & 116.7 & 0.1 & 977.8 & 0.3453407 \\ 86.47 & 203.3 & 0.0007773 & 0.04841 & 2218 & 116.1 & -1.1 & 978.6 & 0.3650592 \\ 86.47 & 201.9 & 0.0007811 & 0.04564 & 2178 & 117 & 0.7 & 977.5 & 0.3358605 \\ 86.47 & 200.4 & 0.0007848 & 0.04316 & 2141 & 117.8 & 2.5 & 976.4 & 0.3087923 \\ 86.47 & 199.5 & 0.0007872 & 0.04166 & 2118 & 118.4 & 3.7 & 975.7 & 0.2919755 \\ 86.47 & 200 & 0.000786 & 0.0424 & 2130 & 118.1 & 3.1 & 976 & 0.300252\end{array}$




\begin{tabular}{|c|c|c|c|c|c|c|c|c|c|c|c|c|c|c|c|}
\hline 6.1 & 125 & 1.3 & 800 & 0.08 & 0.77 & 450 & 86.47 & 204.8 & 0.0007734 & 0.05154 & 2260 & 115.2 & -3.1 & 979.9 & 0.3980046 \\
\hline 5.6 & 126 & 1.3 & 800 & 0.1 & 0.77 & 490 & 86.47 & 202.8 & 0.0007786 & 0.04745 & 2204 & 116.4 & -0.5 & 978.2 & 0.3551921 \\
\hline 5.9 & 130 & 1.3 & 800 & 0.07 & 0.78 & 470 & 86.47 & 203.8 & 0.000776 & 0.04941 & 2231 & 115.8 & -1.7 & 979 & 0.3749421 \\
\hline 5.1 & 131 & 1.3 & 800 & 0.06 & 0.75 & 450 & 86.47 & 204.8 & 0.0007734 & 0.05154 & 2260 & 115.2 & -3.1 & 979.9 & 0.3980046 \\
\hline 13.5 & 195 & 1.3 & 800 & 0.13 & 0.82 & 510 & 86.47 & 201.9 & 0.0007811 & 0.04564 & 2178 & 117 & 0.7 & 977.5 & 0.3358605 \\
\hline 13.7 & 199 & 1.3 & 800 & 0.13 & 0.8 & 500 & 86.47 & 202.3 & 0.0007798 & 0.04653 & 2191 & 116.7 & 0.1 & 977.8 & 0.3453407 \\
\hline 12.3 & 199 & 1.3 & 800 & 0.12 & 0.81 & 490 & 86.47 & 202.8 & 0.0007786 & 0.04745 & 2204 & 116.4 & -0.5 & 978.2 & 0.3551921 \\
\hline 13.5 & 204 & 1.3 & 800 & 0.11 & 0.76 & 490 & 86.47 & 202.8 & 0.0007786 & 0.04745 & 2204 & 116.4 & -0.5 & 978.2 & 0.3551921 \\
\hline 30.9 & 295 & 1.3 & 800 & 0.15 & 0.84 & 490 & 86.47 & 202.8 & 0.0007786 & 0.04745 & 2204 & 116.4 & -0.5 & 978.2 & 0.3551921 \\
\hline 28.4 & 296 & 1.3 & 800 & 0.12 & 0.78 & 510 & 86.47 & 201.9 & 0.0007811 & 0.04564 & 2178 & 117 & 0.7 & 977.5 & 0.3358605 \\
\hline 30.7 & 301 & 1.3 & 800 & 0.15 & 0.8 & 500 & 86.47 & 202.3 & 0.0007798 & 0.04653 & 2191 & 116.7 & 0.1 & 977.8 & 0.3453407 \\
\hline 31.1 & 303 & 1.3 & 800 & 0.12 & 0.78 & 500 & 86.47 & 202.3 & 0.0007798 & 0.04653 & 2191 & 116.7 & 0.1 & 977.8 & 0.3453407 \\
\hline 47.2 & 291 & 1.3 & 800 & 0.17 & 0.85 & 570 & 86.47 & 199 & 0.0007884 & 0.04094 & 2107 & 118.6 & 4.2 & 975.4 & 0.2852313 \\
\hline 48.5 & 406 & 1.3 & 800 & 0.16 & 0.83 & 560 & 86.47 & 199.5 & 0.0007872 & 0.04166 & 2118 & 118.4 & 3.7 & 975.7 & 0.2919755 \\
\hline 49.2 & 406 & 1.3 & 800 & 0.16 & 0.85 & 550 & 86.47 & 200 & 0.000786 & 0.0424 & 2130 & 118.1 & 3.1 & 976 & 0.300252 \\
\hline 4.4 & 118 & 2.6 & 800 & 0.14 & 0.8 & 510 & 86.47 & 201.9 & 0.0007811 & 0.04564 & 2178 & 117 & 0.7 & 977.5 & 0.3358605 \\
\hline 4.7 & 125 & 2.6 & 800 & 0.13 & 0.77 & 520 & 86.47 & 201.4 & 0.0007823 & 0.04478 & 2166 & 117.3 & 1.3 & 977.1 & 0.3265157 \\
\hline 13.2 & 187 & 2.6 & 800 & 0.13 & 0.82 & 530 & 86.47 & 200.9 & 0.0007836 & 0.04396 & 2153 & 117.5 & 1.9 & 976.8 & 0.3175372 \\
\hline 12.9 & 189 & 2.6 & 800 & 0.13 & 0.82 & 560 & 86.47 & 199.5 & 0.0007872 & 0.04166 & 2118 & 118.4 & 3.7 & 975.7 & 0.2919755 \\
\hline 12.6 & 200 & 2.6 & 800 & 0.11 & 0.76 & 550 & 86.47 & 200 & 0.000786 & 0.0424 & 2130 & 118.1 & 3.1 & 976 & 0.300252 \\
\hline 13.5 & 205 & 2.6 & 800 & 0.11 & 0.77 & 540 & 86.47 & 200.4 & 0.0007848 & 0.04316 & 2141 & 117.8 & 2.5 & 976.4 & 0.3087923 \\
\hline 27.8 & 270 & 2.6 & 800 & 0.19 & 0.78 & 560 & 86.47 & 199.5 & 0.0007872 & 0.04166 & 2118 & 118.4 & 3.7 & 975.7 & 0.2919755 \\
\hline 28.9 & 271 & 2.6 & 800 & 0.18 & 0.8 & 580 & 86.47 & 198.8 & 0.0007896 & 0.04025 & 2095 & 118.9 & 4.8 & 975 & 0.2772973 \\
\hline 29.3 & 279 & 2.6 & 800 & 0.18 & 0.74 & 550 & 86.47 & 200 & 0.000786 & 0.0424 & 2130 & 118.1 & 3.1 & 976 & 0.300252 \\
\hline 30.1 & 283 & 2.6 & 800 & 0.17 & 0.79 & 610 & 86.47 & 197.2 & 0.0007931 & 0.0383 & 2063 & 119.7 & 6.4 & 974.1 & 0.2574286 \\
\hline 45.2 & 373 & 2.6 & 800 & 0.22 & 0.7 & 600 & 86.47 & 197.6 & 0.000792 & 0.03893 & 2074 & 119.4 & 5.9 & 974.4 & 0.2634739 \\
\hline 41.9 & 382 & 2.6 & 800 & 0.22 & 0.62 & 620 & 86.47 & 196.8 & 0.0007943 & 0.03769 & 2053 & 119.9 & 6.9 & 973.8 & 0.2514856 \\
\hline 46.5 & 383 & 2.6 & 800 & 0.21 & 0.65 & 580 & 86.47 & 198.8 & 0.0007896 & 0.04025 & 2095 & 118.9 & 4.8 & 975 & 0.2772973 \\
\hline 7 & 125 & 5 & 800 & 0.11 & 0.83 & 510 & 86.47 & 201.9 & 0.0007811 & 0.04564 & 2178 & 117 & 0.7 & 977.5 & 0.3358605 \\
\hline 7.1 & 132 & 5 & 800 & 0.1 & 0.83 & 510 & 86.47 & 201.9 & 0.0007811 & 0.04564 & 2178 & 117 & 0.7 & 977.5 & 0.3358605 \\
\hline 12.8 & 184 & 5 & 800 & 0.1 & 0.81 & 590 & 86.47 & 198.2 & 0.0007908 & 0.03958 & 2085 & 119.2 & 5.3 & 974.7 & 0.2709514 \\
\hline 13.7 & 200 & 5 & 800 & 0.09 & 0.78 & 580 & 86.47 & 198.8 & 0.0007896 & 0.04025 & 2095 & 118.9 & 4.8 & 975 & 0.2772973 \\
\hline 13.7 & 201 & 5 & 800 & 0.09 & 0.77 & 560 & 86.47 & 199.5 & 0.0007872 & 0.04166 & 2118 & 118.4 & 3.7 & 975.7 & 0.2919755 \\
\hline 17.2 & 228 & 5 & 800 & 0.11 & 0.79 & 630 & 86.47 & 196.4 & 0.0007954 & 0.0371 & 2043 & 120.2 & 7.4 & 973.5 & 0.2456444 \\
\hline 30.3 & 270 & 5 & 800 & 0.2 & 0.82 & 600 & 86.47 & 197.6 & 0.000792 & 0.03893 & 2074 & 119.4 & 5.9 & 974.4 & 0.2634739 \\
\hline 29.8 & 273 & 5 & 800 & 0.19 & 0.76 & 600 & 86.47 & 197.6 & 0.000792 & 0.03893 & 2074 & 119.4 & 5.9 & 974.4 & 0.2634739 \\
\hline 29.3 & 278 & 5 & 800 & 0.18 & 0.7 & 600 & 86.47 & 197.6 & 0.000792 & 0.03893 & 2074 & 119.4 & 5.9 & 974.4 & 0.2634739 \\
\hline 26.7 & 283 & 5 & 800 & 0.18 & 0.72 & 590 & 86.47 & 198.2 & 0.0007908 & 0.03958 & 2085 & 119.2 & 5.3 & 974.7 & 0.2709514 \\
\hline 46.5 & 367 & 5 & 800 & 0.27 & 0.77 & 630 & 86.47 & 196.4 & 0.0007954 & 0.0371 & 2043 & 120.2 & 7.4 & 973.5 & 0.2456444 \\
\hline 46.3 & 372 & 5 & 800 & 0.15 & 0.69 & 580 & 86.47 & 198.8 & 0.0007896 & 0.04025 & 2095 & 118.9 & 4.8 & 975 & 0.2772973 \\
\hline 44.7 & 384 & 5 & 800 & 0.15 & 0.66 & 610 & 86.47 & 197.2 & 0.0007931 & 0.0383 & 2063 & 119.7 & 6.4 & 974.1 & 0.2574286 \\
\hline
\end{tabular}

Smooth $1 / 2$ inch, evaporation R134a with SUS169 ester-m oil

$\begin{array}{lllllll}3.34 & 91 & 1.3 & 500 & 0.12 & 0.85 & 306 \\ 3.33 & 89 & 1.3 & 500 & 0.12 & 0.89 & 302 \\ 6.49 & 128 & 1.3 & 500 & 0.11 & 0.78 & 314 \\ 7 & 124 & 1.3 & 500 & 0.12 & 0.84 & 304 \\ 14.76 & 192 & 1.3 & 500 & 0.08 & 0.84 & 311 \\ 15.57 & 193 & 1.3 & 500 & 0.08 & 0.84 & 305 \\ 26.73 & 265 & 1.3 & 500 & 0.12 & 0.81 & 339 \\ 26.73 & 268 & 1.3 & 500 & 0.11 & 0.79 & 338 \\ 3.69 & 88 & 2.3 & 500 & 0.13 & 0.84 & 313 \\ 3.34 & 89 & 2.3 & 500 & 0.13 & 0.84 & 313 \\ 8.09 & 126 & 2.3 & 500 & 0.1 & 0.85 & 303 \\ 8.47 & 126 & 2.3 & 500 & 0.09 & 0.78 & 317 \\ 18.96 & 187 & 2.3 & 500 & 0.09 & 0.85 & 300 \\ 18.96 & 187 & 2.3 & 500 & 0.09 & 0.85 & 301 \\ 26.77 & 242 & 2.3 & 500 & 0.15 & 0.83 & 354 \\ 26.74 & 240 & 2.3 & 500 & 0.15 & 0.84 & 353 \\ 6.83 & 89 & 4.9 & 500 & 0.12 & 0.84 & 291 \\ 6.39 & 88 & 4.9 & 500 & 0.11 & 0.85 & 291 \\ 9.05 & 131 & 4.9 & 500 & 0.13 & 0.81 & 329 \\ 10.65 & 136 & 4.9 & 500 & 0.12 & 0.82 & 308 \\ 8.93 & 136 & 4.9 & 500 & 0.11 & 0.77 & 319 \\ 20.74 & 203 & 4.9 & 500 & 0.09 & 0.82 & 325 \\ 21.76 & 205 & 4.9 & 500 & 0.1 & 0.83 & 310 \\ 21.35 & 205 & 4.9 & 500 & 0.1 & 0.83 & 309 \\ 28.79 & 239 & 4.9 & 500 & 0.12 & 0.81 & 311 \\ 29.41 & 236 & 4.9 & 500 & 0.13 & 0.82 & 312\end{array}$
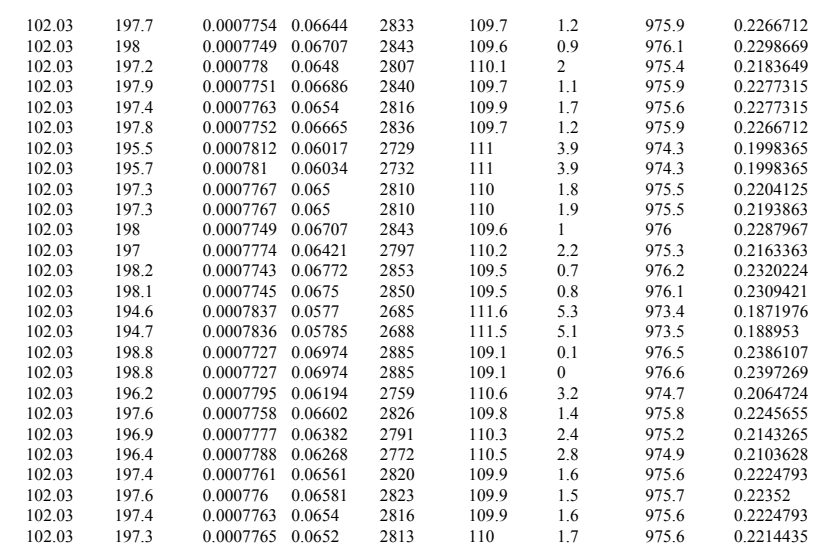

Smooth 1/2 inch, condensation

R134a with SUS169 ester-m oil

\begin{tabular}{|c|c|c|c|c|c|}
\hline 0.23 & 89 & 1.3 & 500 & 0.82 & 0.08 \\
\hline 0.24 & 88 & 1.3 & 500 & 0.82 & 0.09 \\
\hline 0.46 & 122 & 1.3 & 500 & 0.84 & 0.12 \\
\hline 0.62 & 123 & 1.3 & 500 & 0.83 & 0.14 \\
\hline 1.32 & 213 & 1.3 & 500 & 0.82 & 0.1 \\
\hline 1.24 & 212 & 1.3 & 500 & 0.83 & 0.11 \\
\hline 2.44 & 300 & 1.3 & 500 & 0.8 & 0.12 \\
\hline 2.32 & 299 & 1.3 & 500 & 0.8 & 0.14 \\
\hline 0.36 & 92 & 2.3 & 500 & 0.82 & 0.09 \\
\hline 0.44 & 92 & 2.3 & 500 & 0.82 & 0.09 \\
\hline 0.68 & 125 & 2.3 & 500 & 0.85 & 0.14 \\
\hline 0.74 & 125 & 2.3 & 500 & 0.84 & 0.13 \\
\hline 1.26 & 202 & 2.3 & 500 & 0.8 & 0.11 \\
\hline 1.18 & 203 & 2.3 & 500 & 0.79 & 0.1 \\
\hline 3.09 & 308 & 2.3 & 500 & 0.8 & 0.15 \\
\hline 3.49 & 306 & 2.3 & 500 & 0.8 & 0.16 \\
\hline 0.25 & 92 & 4.9 & 500 & 0.84 & 0.12 \\
\hline 0.35 & 92 & 4.9 & 500 & 0.84 & 0.11 \\
\hline 0.8 & 125 & 4.9 & 500 & 0.83 & 0.14 \\
\hline 0.95 & 124 & 4.9 & 500 & 0.84 & 0.13 \\
\hline
\end{tabular}

Micro-fin 1/2 inch, evaporation R134a with SUS169 ester-m oil
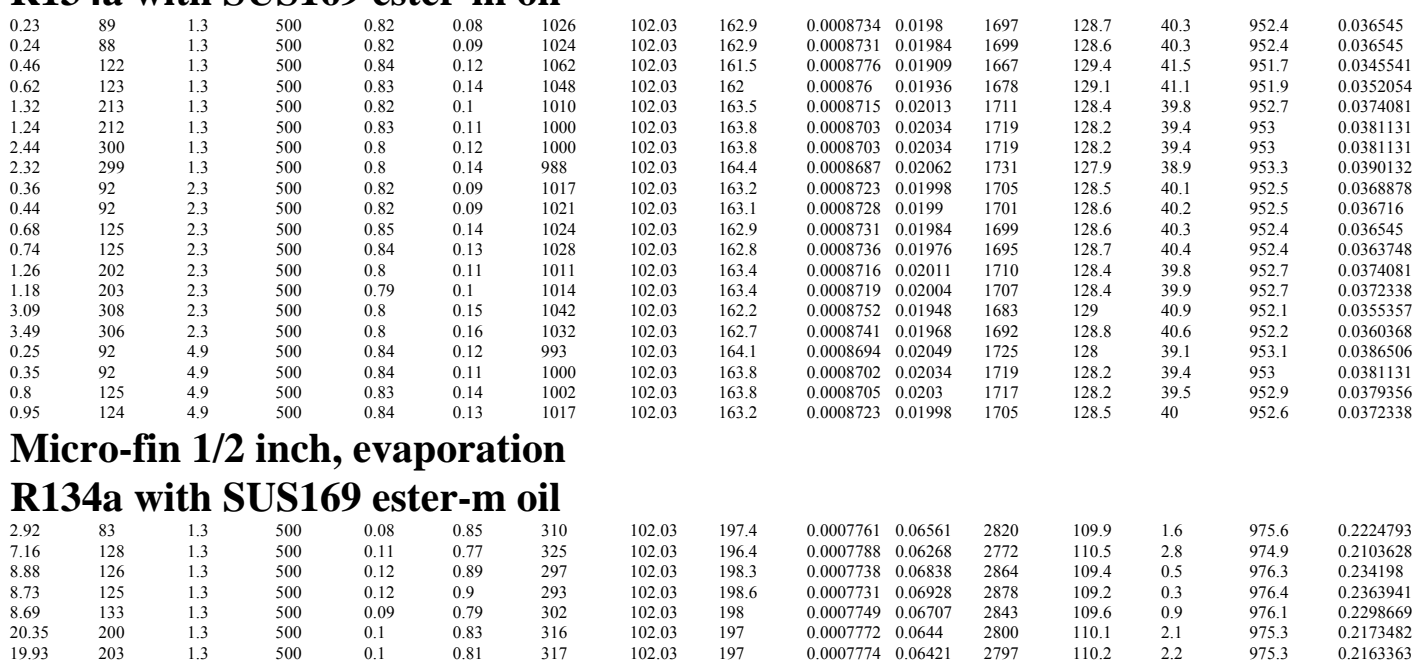


\begin{tabular}{|c|c|c|c|c|c|c|c|c|c|c|c|c|c|c|c|}
\hline 29.28 & 258 & 1.3 & 500 & 0.11 & 0.78 & 342 & 102.03 & 195.3 & 0.0007817 & 0.05966 & 2720 & 111.1 & 4.2 & 974.1 & 0.1970583 \\
\hline 29.82 & 260 & 1.3 & 500 & 0.1 & 0.76 & 343 & 102.03 & 195.3 & 0.0007819 & 0.05949 & 2717 & 111.2 & 4.4 & 974 & 0.1952276 \\
\hline 4.27 & 91 & 2.3 & 500 & 0.14 & 0.83 & 317 & 102.03 & 197 & 0.0007774 & 0.06421 & 2797 & 110.2 & 2.2 & 975.3 & 0.2163363 \\
\hline 3.96 & 91 & 2.3 & 500 & 0.14 & 0.82 & 319 & 102.03 & 196.9 & 0.0007777 & 0.06382 & 2791 & 110.3 & 2.3 & 975.2 & 0.215329 \\
\hline 9.14 & 131 & 2.3 & 500 & 0.08 & 0.85 & 313 & 102.03 & 197.3 & 0.0007767 & 0.065 & 2810 & 110 & 1.8 & 975.5 & 0.2204125 \\
\hline 9.11 & 132 & 2.3 & 500 & 0.08 & 0.86 & 313 & 102.03 & 197.3 & 0.0007767 & 0.065 & 2810 & 110 & 1.8 & 975.5 & 0.2204125 \\
\hline 18.84 & 186 & 2.3 & 500 & 0.1 & 0.84 & 319 & 102.03 & 196.9 & 0.0007777 & 0.06382 & 2791 & 110.3 & 2.3 & 975.2 & 0.215329 \\
\hline 19.35 & 185 & 2.3 & 500 & 0.1 & 0.87 & 317 & 102.03 & 197 & 0.0007774 & 0.06421 & 2797 & 110.2 & 2.2 & 975.3 & 0.2163363 \\
\hline 27.61 & 242 & 2.3 & 500 & 0.15 & 0.83 & 352 & 102.03 & 194.7 & 0.0007834 & 0.05801 & 2691 & 111.5 & 5 & 973.6 & 0.1898369 \\
\hline 27.95 & 244 & 2.3 & 500 & 0.14 & 0.82 & 351 & 102.03 & 194.8 & 0.0007832 & 0.05817 & 2694 & 111.5 & 5 & 973.6 & 0.1898369 \\
\hline 2.86 & 84 & 4.9 & 500 & $\begin{array}{l}0.09 \\
0.09\end{array}$ & 0.86 & 322 & 102.03 & $\begin{array}{l}196.0 \\
196.7\end{array}$ & 0.0007783 & 0.06324 & 2781 & 110.4 & 2.6 & 975 & $\begin{array}{l}0.18123354 \\
0.212354\end{array}$ \\
\hline 2.84 & 84 & 4.9 & 500 & 0.09 & 0.81 & 325 & 102.03 & 196.4 & 0.0007788 & 0.06268 & 2772 & 110.5 & 2.9 & 974.9 & 0.2093834 \\
\hline $\begin{array}{l}8.04 \\
8.96\end{array}$ & $\begin{array}{l}04 \\
125\end{array}$ & $\begin{array}{l}4.9 \\
4.9\end{array}$ & 500 & 0.1 & 0.86 & 320 & 102.03 & $\begin{array}{l}19.4 \\
196.8\end{array}$ & 0.0007779 & 0.06363 & 2787 & 110.3 & 2.4 & 975.2 & 0.2143265 \\
\hline 9.43 & 126 & 4.9 & 500 & 0.11 & 0.89 & 313 & 102.03 & 197.3 & 0.0007767 & 0.065 & 2810 & 110 & 1.8 & 975.5 & 0.2204125 \\
\hline 18.44 & 197 & 4.9 & 500 & 0.1 & 0.79 & 337 & 102.03 & 195.7 & 0.0007809 & 0.06052 & 2735 & 111 & 3.8 & 974.3 & 0.2007713 \\
\hline 18.47 & 196 & 4.9 & 500 & 0.11 & 0.8 & 343 & 102.03 & 195.3 & 0.0007819 & 0.05949 & 2717 & 111.2 & 4.3 & 974 & 0.1961408 \\
\hline 30.93 & 238 & 4.9 & 500 & 0.11 & 0.89 & 362 & 102.03 & 194.1 & 0.0007851 & 0.05646 & 2663 & 111.9 & 5.9 & 973.1 & 0.1820286 \\
\hline 30.9 & 236 & 4.9 & 500 & 0.11 & 0.89 & 358 & 102.03 & 194.3 & 0.0007844 & 0.05707 & 2674 & 111.7 & 5.6 & 973.2 & 0.184595 \\
\hline
\end{tabular}

Micro-fin 1/2 inch, condensation

R134a with SUS169 ester-m oil

\begin{tabular}{|c|c|c|c|c|c|c|c|c|c|c|c|c|c|c|c|}
\hline 0.17 & 85 & 1.3 & 500 & 0.85 & 0.09 & 1049 & 102.03 & 162 & 0.0008761 & 0.01934 & 1678 & 129.1 & 41.2 & 951.9 & 0.0350415 \\
\hline 0.19 & 85 & 1.3 & 500 & 0.85 & 0.09 & 1057 & 102.03 & 161.6 & 0.000877 & 0.01918 & 1671 & 129.3 & 41.4 & 951.8 & 0.0347158 \\
\hline 0.56 & 127 & 1.3 & 500 & 0.82 & 0.09 & 1041 & 102.03 & 162.3 & 0.0008751 & 0.0195 & 1684 & 129 & 40.8 & 952.1 & 0.0357019 \\
\hline 0.6 & 127 & 1.3 & 500 & 0.82 & 0.1 & 1041 & 102.03 & 162.3 & 0.0008751 & 0.0195 & 1684 & 129 & 40.9 & 952.1 & 0.0355357 \\
\hline 1.75 & 198 & 1.3 & 500 & 0.85 & 0.12 & 1028 & 102.03 & 162.8 & 0.0008736 & 0.01976 & 1695 & 128.7 & 40.4 & 952.4 & 0.0363748 \\
\hline 1.89 & 199 & 1.3 & 500 & 0.85 & 0.13 & 1024 & 102.03 & 162.9 & 0.0008731 & 0.01984 & 1699 & 128.6 & 40.3 & 952.4 & 0.036545 \\
\hline 4.68 & 302 & 1.3 & 500 & 0.81 & 0.15 & 994 & 102.03 & 164.1 & 0.0008696 & 0.02047 & 1724 & 128 & 39.1 & 953.1 & 0.0386506 \\
\hline 4.31 & 304 & 1.3 & 500 & 0.8 & 0.13 & 1004 & 102.03 & 163.7 & 0.0008708 & 0.02026 & 1716 & 128.2 & 39.6 & 952.8 & 0.037759 \\
\hline 4.23 & 306 & 1.3 & 500 & 0.79 & 0.14 & 1001 & 102.03 & 163.9 & 0.0008704 & 0.02032 & 1718 & 128.2 & 39.4 & 953 & 0.0381131 \\
\hline 0.16 & 84 & 2.3 & 500 & 0.84 & 0.1 & 1068 & 102.03 & 161.2 & 0.0008783 & 0.01897 & 1662 & 129.5 & 41.8 & 951.5 & 0.0340736 \\
\hline 0.14 & 84 & 2.3 & 500 & 0.84 & 0.11 & 1055 & 102.03 & 161.7 & 0.0008786 & 0.01922 & 1673 & 129.3 & 41.3 & 951.8 & 0.0348783 \\
\hline 0.63 & 122 & 2.3 & 500 & 0.83 & 0.12 & 1056 & 102.03 & 161.7 & 0.0008769 & 0.0192 & 1672 & 129.3 & 41.4 & 951.8 & 0.0347158 \\
\hline 0.74 & 127 & 2.3 & 500 & 0.82 & 0.11 & 1020 & 102.03 & 163.1 & 0.0008726 & 0.01992 & 1702 & 128.6 & 40.1 & 952.5 & 0.0368878 \\
\hline 1.99 & 203 & 2.3 & 500 & 0.81 & 0.07 & 1018 & 102.03 & 163.1 & 0.0008724 & 0.01996 & 1704 & 128.5 & 40 & 952.6 & 0.0370604 \\
\hline 2.06 & 201 & 2.3 & 500 & 0.82 & 0.09 & 1015 & 102.03 & 163.3 & 0.0008721 & 0.02002 & 1706 & 128.5 & 40 & 952.6 & 0.0370604 \\
\hline 4.62 & 298 & 2.3 & 500 & 0.81 & 0.13 & 1028 & 102.03 & 162.8 & 0.0008736 & 0.01976 & 1695 & 128.7 & 40.4 & 952.4 & 0.0363748 \\
\hline 4.79 & 305 & 2.3 & 500 & 0.78 & 0.1 & 1002 & 102.03 & 163.8 & 0.0008705 & 0.0203 & 1717 & 128.2 & 39.5 & 952.9 & 0.0379356 \\
\hline 5.06 & 307 & 2.3 & 500 & 0.79 & 0.1 & 1017 & 102.03 & 163.2 & 0.0008723 & 0.01998 & 1705 & 128.5 & 40 & 952.6 & 0.0370604 \\
\hline 0.27 & 94 & 4.9 & 500 & 0.85 & 0.07 & 1000 & 102.03 & 163.8 & 0.0008703 & 0.02034 & 1719 & 128.2 & 39.4 & 953 & 0.0381131 \\
\hline 0.33 & 93 & 4.9 & 500 & 0.86 & 0.08 & 993 & 102.03 & 164.1 & 0.0008694 & 0.02049 & 1725 & 128 & 39.1 & 953.1 & 0.0386506 \\
\hline 0.88 & 127 & 4.9 & 500 & 0.87 & 0.08 & 997 & 102.03 & 164 & 0.0008699 & 0.02041 & 1722 & 128.1 & 39.3 & 953 & 0.0382915 \\
\hline 0.78 & 127 & 4.9 & 500 & 0.87 & 0.08 & 993 & 102.03 & 164.1 & 0.0008694 & 0.02049 & 1725 & 128 & 39.1 & 953.1 & 0.0386506 \\
\hline 1.72 & 206 & 4.9 & 500 & 0.85 & 0.09 & 974 & 102.03 & 164.8 & 0.0008673 & 0.02089 & 1741 & 127.7 & 38.3 & 953.6 & 0.0401213 \\
\hline 3.99 & 289 & 4.9 & 500 & 0.78 & 0.08 & 974 & 102.03 & 164.8 & 0.0008673 & 0.02089 & 1741 & 127.7 & 38.4 & 953.6 & 0.0399345 \\
\hline 3.73 & 290 & 4.9 & 500 & 0.81 & 0.06 & 1013 & 102.03 & 163.4 & 0.0008718 & 0.02007 & 1708 & 128.4 & 39.9 & 952.7 & 0.0372338 \\
\hline
\end{tabular}

